Aus dem Institut für Pathologie

(Prof. Dr. med. P. Ströbel)

der Medizinischen Fakultät der Universität Göttingen

\title{
Comparative Morphologic and Molecular Classification of Neuroendocrine Tumors of the Thymus in Humans
}

\author{
INAUGURAL - DISSERTATION \\ zur Erlangung des Doktorgrades \\ der Medizinischen Fakultät der \\ Georg-August-Universität zu Göttingen
}

vorgelegt von

Helen Dinter

aus

Berlin 
Dekan:

\section{Betreuungsausschuss}

Betreuer/in:

Ko-Betreuer/in:

\section{Prüfungskommission}

Referent/in:

Prof. Dr. med. Philipp Ströbel

Ko-Referent/in:

Drittreferent/in:
Prof. Dr. med. W. Brück

Prof. Dr. med. Philipp Ströbel

Prof. Dr. med. Volker Ellenrieder

Datum der mündlichen Prüfung: $\quad$ 28.04.2021 
Hiermit erkläre ich, die Dissertation mit dem Titel "Comparative Morphologic and Molecular Classification of Neuroendocrine Tumors of the Thymus in Humans" eigenständig angefertigt und keine anderen als die von mir angegebenen Quellen und Hilfsmittel verwendet zu haben.

Göttingen, den 
Die Daten, auf denen die vorliegende Arbeit basiert, wurden teilweise publiziert:

Bohnenberger H, Dinter H, König A, Ströbel P (2017): Neuroendocrine tumors of the thymus and mediastinum. J Thorac Dis $\underline{9}$, S1448-S1457

Dinter H, Bohnenberger H, Beck J, Bornemann-Kolatzki K, Küffer, S, Klein L, Franks T, Roden A, Emmert A, Hinterthaner M, et al. (2019): Molecular Classification of Neuroendocrine Tumors of the Thymus. Journal of Thoracic Oncology. doi: 10.1016/j.jtho.2019.04.015. [Epub ahead of print].

Dinter H, Bohnenberger H, Küffer S, Brcic L, Popper H, Marino M, Franks T, Beck J, Ekkehard S, Weiss C, Marx A, Ströbel P: Über die Anwendbarkeit des ENETS Grading Systems bei Neuroendokrinen Tumoren des Thymus (TNET), 101. Jahrestagung der Deutschen Gesellschaft für Pathologie, Erlangen, 2017

Pelosi G, Bianchi F, Hofman P, Pattini L, Ströbel P, Calabrese F, Naheed S, Holden $\mathrm{C}$, Cave J, Bohnenberger $\mathrm{H}$, et al. (2019): Recent advances in the molecular landscape of lung neuroendocrine tumors. Expert Rev Mol Diagn 19, 281-297 


\section{Table of Contents}

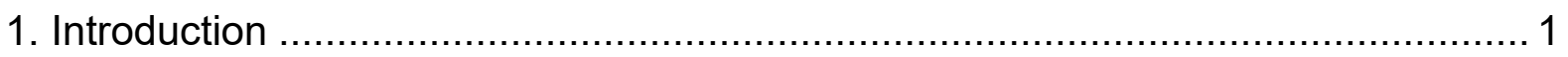

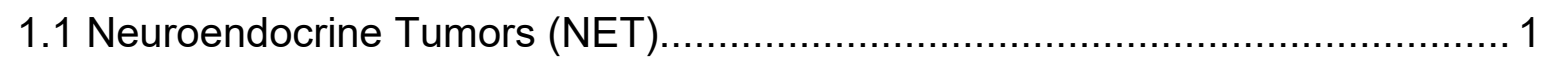

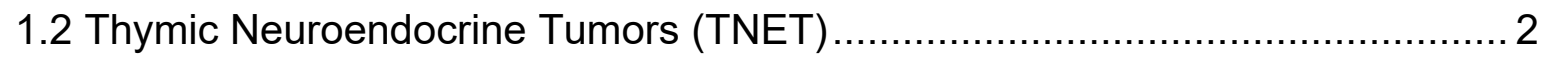

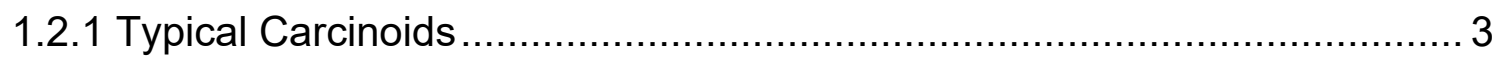

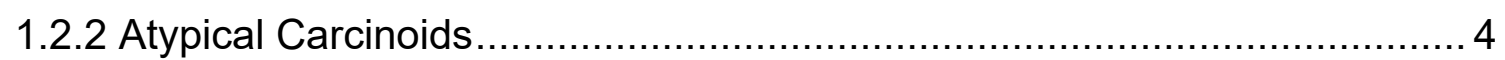

1.2.3 Large Cell Neuroendocrine Carcinoma ................................................ 4

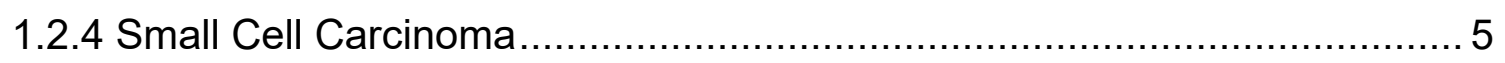

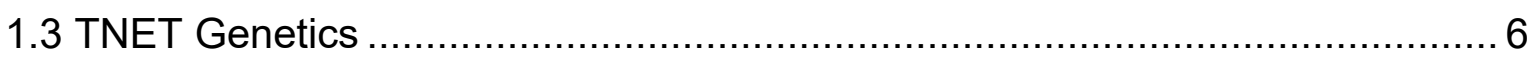

1.4 WHO Grading of Gastro-Intestinal and Pancreatic NET .............................. 7

1.5 Nomenclature and Grading Controversies.............................................. 7

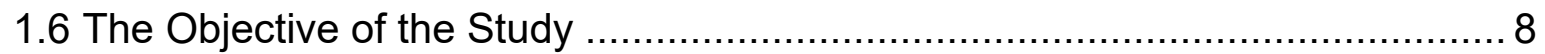

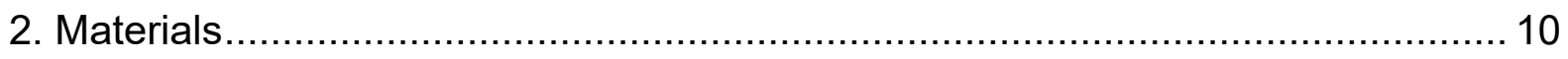

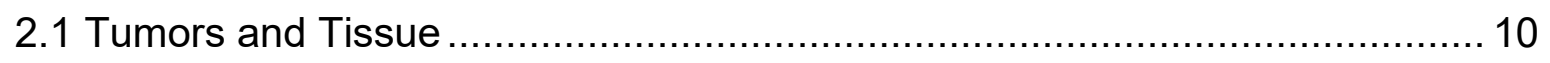

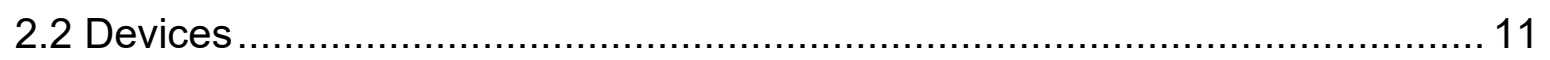

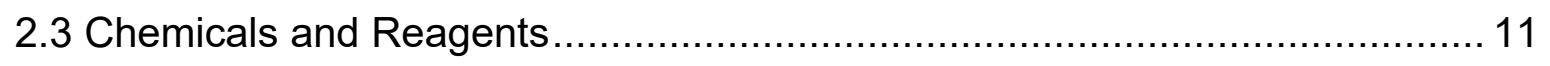

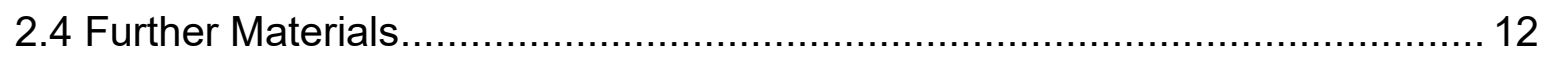

2.5 Computer Software and Internet Addresses .......................................... 13

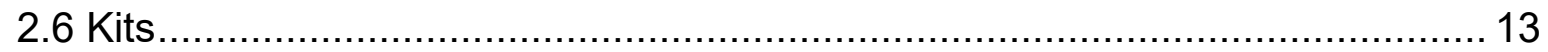

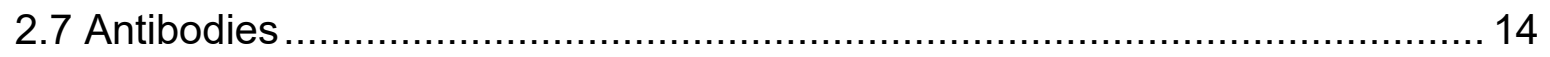

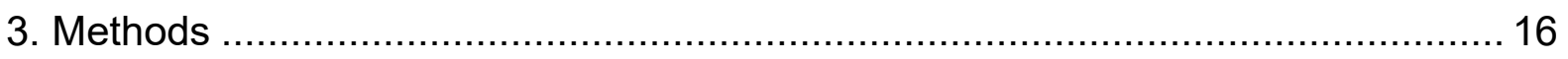

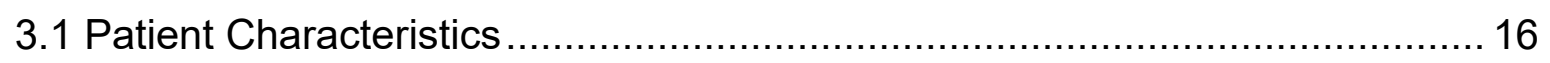

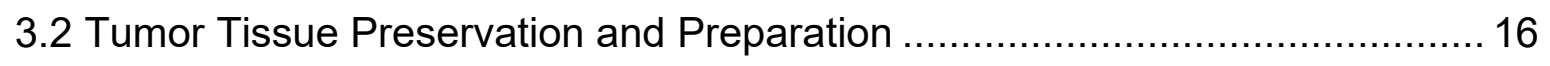

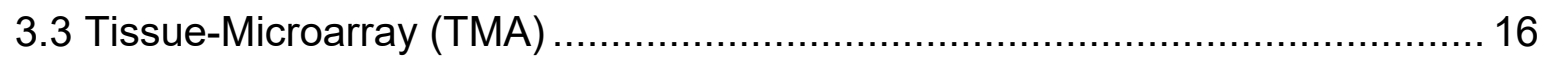

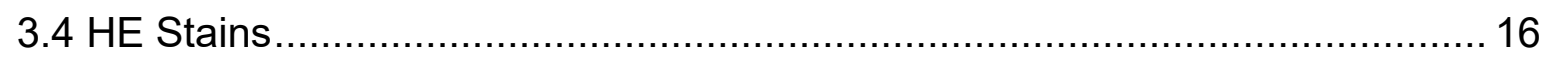

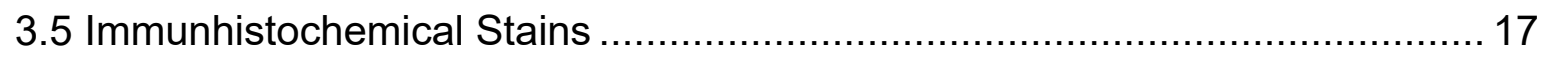

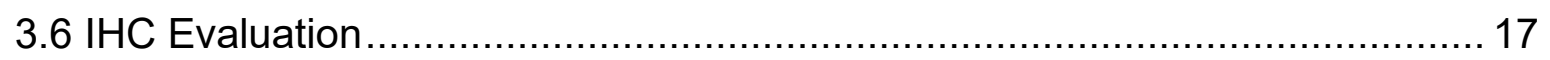

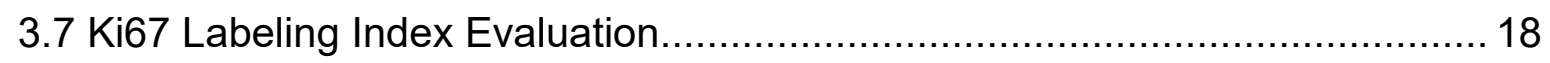

3.8 WHO, ENETS, and Pelosi et al. Classification............................................ 18

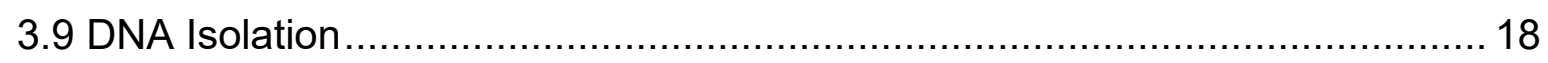

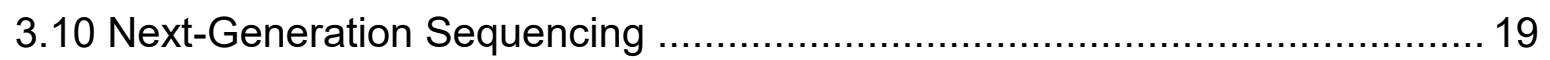

3.11 Shallow Whole Genome Sequencing by Chronix $\mathrm{GmbH}$.......................... 20

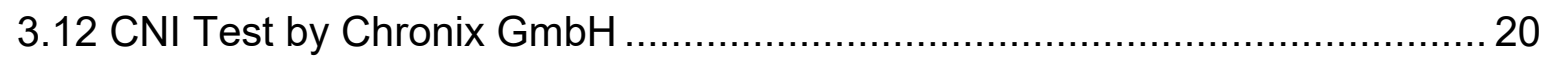

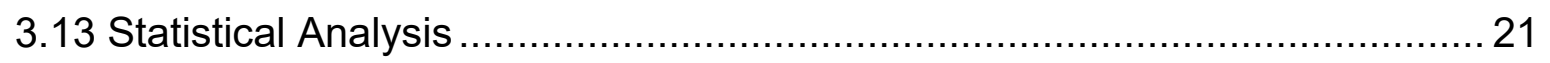

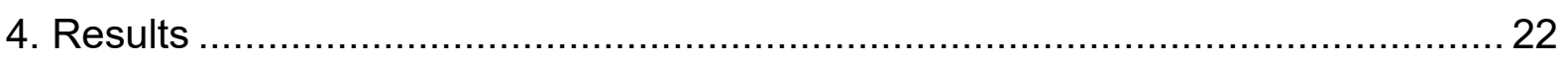

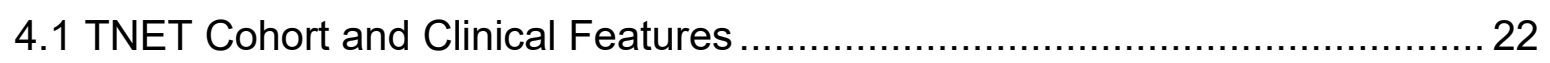


4.2 Relationship of Proliferation Markers with Overall Survival.......................... 23

4.2.1 HE and Ki67 Slide Preparation...................................................... 23

4.2.2 Correlation Analysis of Mitoses, Ki67, Necrosis and Survival ................. 24

4.2.3 Ki67 of WHO Diagnosis and Correlation with Mitotic Count ..................... 24

4.3 A Comparison of Three Classification Systems ..................................... 26

4.3.1 The ENETS Classification and Pelosi NET Grading Proposal ................. 26

4.3.2. Applying the ENETS and Pelosi et al. Classifications .......................... 27

4.3.3 Survival Analysis of Three Grading Systems ...................................... 28

4.3.4 Shallow Whole Genome Sequencing and Copy Number Instability Score

4.3.5 Three Grading Systems Correlated with Genetic Aberrations ................. 32

4.3.6 Genetic Alterations Compared to Mitotic Count, Ki67, and Survival......... 34

4.4 Clustering Analyses of Shallow Whole Genome Sequencing Data ................ 36

4.5 NET G3 versus NEC in Thymic NET ............................................... 38

4.5.1 The Immunohistochemical Profile of NET G3 ................................... 38

4.5.2 NET G3 versus NEC Immunohistochemistry and Histologic Features..... 39

4.5.3 EZH2 and Chromogranin A in LCNEC ............................................. 39

4.5.4 Next-Generation Sequencing of Five Genes..................................... 40

4.6 Calculating Cutoffs with Cox Proportional Hazard Regressions ................... 41

4.6.1 Cox Hazard Regressions and Survival Analysis in Statistica .................. 41

4.6.2 Mitotic Count Cutoffs and Survival Analysis ....................................... 41

4.6.3 Ki67 Cutoffs and Survival Analysis.............................................. 43

4.6.4 CNI Cutoffs and Survival Analysis.................................................... 44

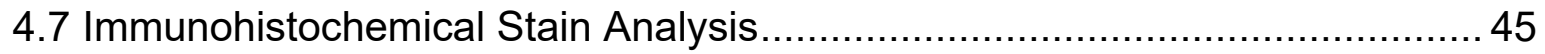

4.7.1 Tissue-Microarray and Evaluation of Stains ..................................... 45

4.7.2 ATRX Immunohistochemical Analysis........................................... 48

4.7.3 DAXX Immunohistochemical Analysis ......................................... 50

4.7.4 SSTR2A Immunohistochemical Analysis .......................................... 52

4.7.5 RB1 Immunohistochemical Analysis ........................................... 53

4.7.6 P53 Immunohistochemical Analysis ............................................... 55

4.7.7 C-Myc Immunohistochemical Analysis ........................................... 57

4.7.8 SDHA Immunohistochemical Analysis ............................................ 59

4.7.9 SDHB Immunohistochemical Analysis .......................................... 61

4.7.10 EZH2 Immunohistochemical Analysis .......................................... 63

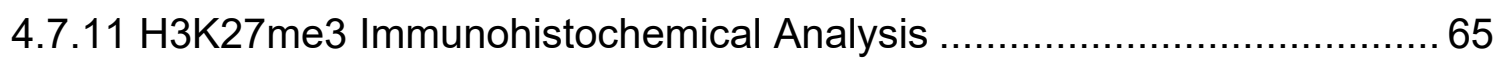

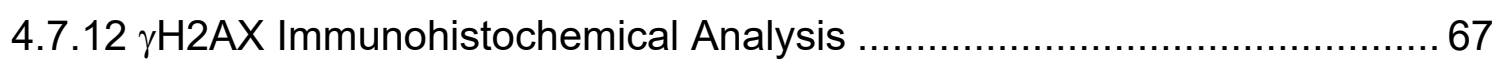

4.7.13 Chromogranin A Immunohistochemical Analysis ............................69 
4.7.14 Keratin Immunohistochemical Analysis ........................................... 71

4.7.15 Calcitonin Immunohistochemical Analysis......................................... 73

4.7.16 YAP1 Immunohistochemical Analysis ............................................... 74

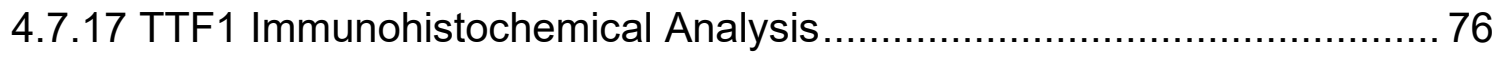

4.7.18 PAX8 Immunohistochemical Analysis .............................................. 77

4.7.19 Serotonin Immunohistochemical Analysis ......................................... 79

4.7.20 CDX2 Immunohistochemical Analysis ............................................... 79

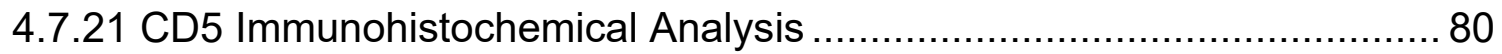

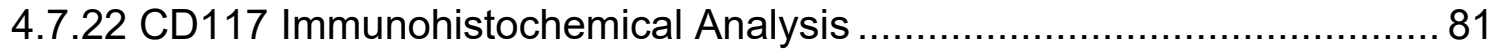

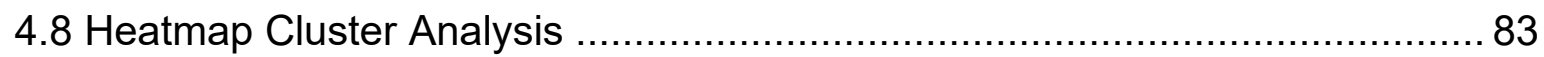

4.9 Evidence of Morphologic and Genomic Progression in TNET ..................... 86

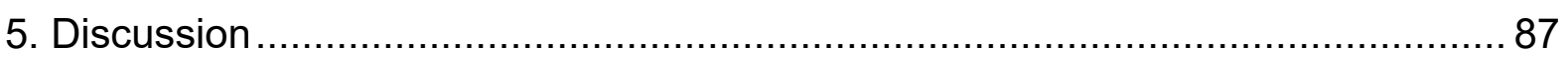

5.1 NET Classification Systems, Survival and Genomic Findings ….................. 87

5.2 CNI Cluster Analysis Reveals Three Major Molecular Clusters ..................... 89

5.2.1 EZH2 and Chromogranin A Associate with Genomic Instability ............... 90

5.3 WHO Large Cell Neuroendocrine Carcinoma Comprise a NET G3 Subgroup92

5.3.1 Targeted Sequencing Revealed Unexpected Mutations in LCNEC .........93

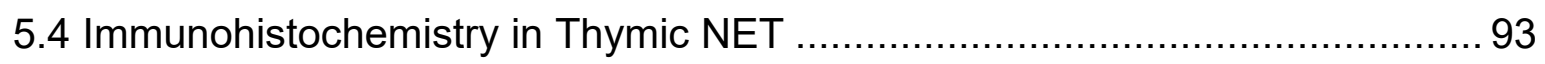

5.5 Morphologic and Genomic Progression in TNET ....................................... 94

5.6 Proposal for an Integrated "Morpho-Molecular" TNET Classification .............. 95

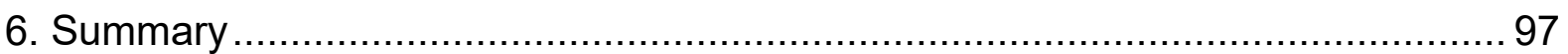

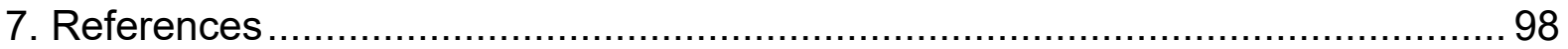




\section{List of Abbreviations}

\begin{tabular}{|c|c|}
\hline$A C$ & atypical carcinoid \\
\hline ANP & atrial natriuretic peptide \\
\hline ATRX & alpha thalassemia mental retardation $\mathrm{x}$-linked \\
\hline $\mathrm{CgA}$ & chromogranin A \\
\hline $\mathrm{CGH}$ & comparative genomic hybridization \\
\hline CNA & chromosomal number alterations \\
\hline CNI score & copy number instability score \\
\hline DAXX & death domain-associated protein \\
\hline ENETS & European neuroendocrine tumor society \\
\hline $\mathrm{EZH} 2$ & enhancer of zeste 2 polycomb complex 2 \\
\hline FFPE & formalin-fixed paraffin-embedded \\
\hline GEP NET & gastro-entero-pancreatic neuroendocrine tumors \\
\hline $\mathrm{HE}$ & hematoxylin eosin \\
\hline HGNEC & high-grade neuroendocrine carcinoma \\
\hline HPF & high-power field \\
\hline $\mathrm{IHC}$ & immunohistochemistry \\
\hline Ki67 LI & ki67 labeling index \\
\hline LCNEC & large cell neuroendocrine carcinoma \\
\hline $\mathrm{LOH}$ & loss of heterozygosity \\
\hline NEC & neuroendocrine carcinoma \\
\hline NET & neuroendocrine tumors \\
\hline NGS & next-generation sequencing \\
\hline NSE & neuron-specific enolase \\
\hline PAX8 & paired box protein \\
\hline PCR2 & polycomb repressor complex 2 \\
\hline PNET & pulmonary neuroendocrine tumors \\
\hline SCC & small-cell neuroendocrine carcinoma \\
\hline SCLC & small-cell lung cancer \\
\hline $\mathrm{SDH}$ & succinate dehydrogenase \\
\hline TC & typical carcinoid \\
\hline TMA & tissue microarray \\
\hline TNET & thymic neuroendocrine tumors \\
\hline TTF1 & thymic transcription factor 1 \\
\hline WGS & whole-genome sequencing \\
\hline WHO & World Health Organization \\
\hline YAP1 & yes-associated protein 1 \\
\hline
\end{tabular}




\section{Introduction}

\subsection{Neuroendocrine Tumors (NET)}

Neuroendocrine tumors are complex and multifaceted epithelial neoplasms that can arise in almost any organ of the body (Suster and Moran 1995; Moran and Suster 2000a; Moran 2005; Ströbel et al. 2014). The neoplasms originate from the diffuse neuroendocrine cell system, a minor cell population scattered throughout every healthy human body (Kaltsas et al. 2004; Ströbel et al. 2014).

Put simply, neuroendocrine cells receive neuronal input via neurotransmitters and, as a result, release (glyco)peptides and monoamines into the blood stream (Kim and Hong 2016). Accordingly, the cancer cells contain neurosecretory, dense core granules (visible with an electron microscope) and can over-secrete different hormones (Reid et al. 2014). This may lead to certain hormonal syndromes. In pathology, the cancer cells are diagnosed by the expression of the neuroendocrine markers chromogranin A, synaptophysin, CD56, and neuron-specific enolase (Klimstra et al. 2010; Klimstra et al. 2015; Marx et al. 2015a).

Neuroendocrine tumors account for only $0.5 \%$ of all malignancies, but their prevalence has been increasing over the past few decades due to better diagnostic technologies, an increased use of immunohistochemistry, and an increased awareness of NET (Modlin et al. 2003; Frilling et al. 2012; Oronsky et al. 2017). The most common location for NET is in the gastro-entero-pancreatic (GEP) system constituting about $65 \%$ of all NET, followed by the lungs (Modlin et al. 2008). Only very few cases occur in the thymus. The anatomical location and clinical presentation are among the most important prognostic variables in NET (Sorbye et al. 2013; llett et al. 2015).

Nonetheless, all NET regardless of location, can be categorized into three grades: well-differentiated NET G1, intermediate-differentiated NET G2, and the (often) poorlydifferentiated NEC G3, according to the WHO classification (Rindi et al. 2007; Pelosi et al. 2014; Marx et al. 2015a; Travis et al. 2015). However, the formal criteria and cutoffs differ amongst most organ systems. Pulmonary and thymic NET, also referred to as foregut NET, are classified identically, by means of mitotic rate, the presence or lack of necrosis and tumor morphology (Ullmann et al. 2002). GEP-NET however, can be classified according to the ki67 labeling index or mitotic count and necrosis, and morphology (Rindi et al. 2007; Pelosi et al. 2014; Marx et al. 2015a; Travis et al. 2015). 
Despite these conceptual differences, NET show very few organ-specific morphological traits (Klimstra et al. 2015).

\subsection{Thymic Neuroendocrine Tumors (TNET)}

Thymic neuroendocrine tumors are a very rare subgroup of NET with an unpredictable clinical behavior (Gal et al. 2001). They make up $<5 \%$ of all mediastinal and thymic neoplasms and comprise only $0.4 \%$ of all neuroendocrine tumors (Ströbel et al. 2014; Weissferdt et al. 2014; Filosso et al. 2015). Nevertheless, these tumors are very aggressive malignancies, metastasizing in $70 \%$ of TNET patients, with a 5-year survival rate of only $28 \%$ (Gaur et al. 2010). The limited data available on TNET is based on small retrospective studies, and underlines that these tumors make up their own unique entity, with distinct etiology (i.e. cigarette smoking), epidemiology, and genetic makeup (Bohnenberger et al. 2017). However, as mentioned above, TNET share their nomenclature and grading system with their pulmonary counterparts.

The most recent edition of the WHO 2015 grading system organizes TNET (and PNET) into low-grade typical carcinoids (TC), intermediate-grade atypical carcinoids (AC), and high-grade large cell neuroendocrine carcinomas (LCNEC) and small cell carcinomas (SCC) (Marx et al. 2015a; Ma et al. 2017) (Fig. 1).

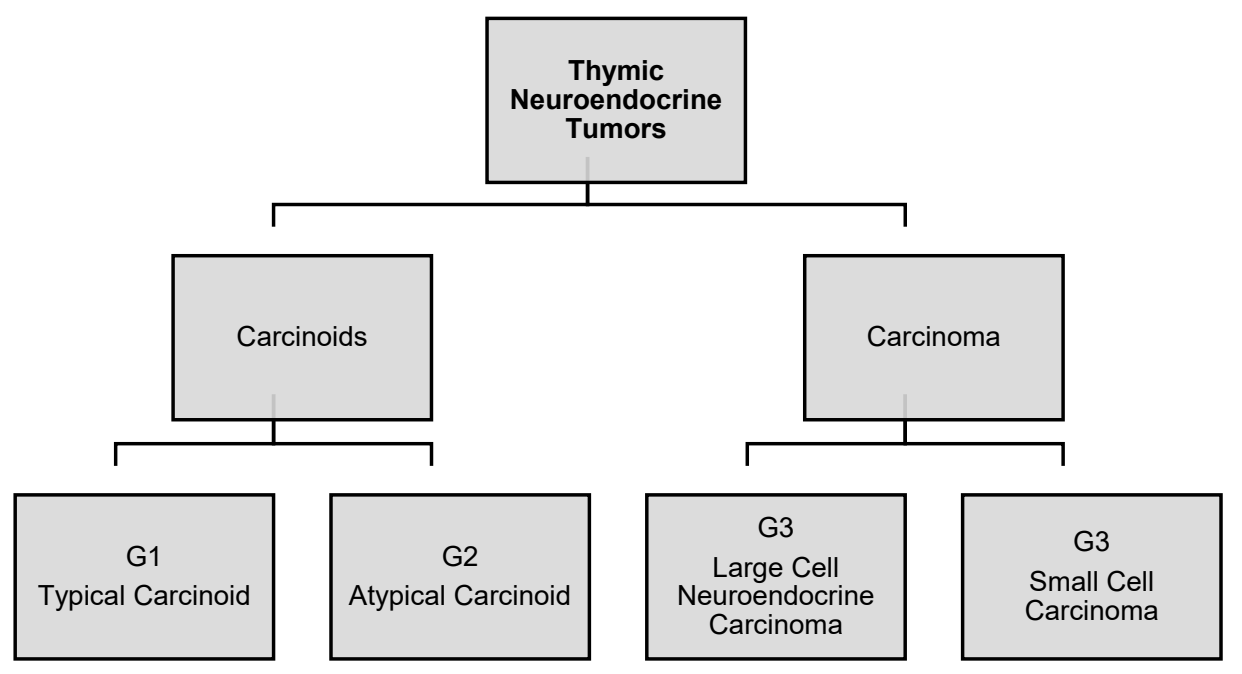

Figure 1: World Health Organization classification of thymic neuroendocrine tumors.

The classification is based on morphology, mitotic rate, and the presence or lack of necrosis. (Edge 2010; Pelosi et al. 2017a). Low- and intermediate-grade TC and AC, and high-grade LCNEC and SCC have been thought to be unrelated entities, and not to result from succeeding stages of de-differentiation (Ströbel et al. 2014). However, 
emerging data suggests that at least some secondary high-grade NET may develop from pre-existing carcinoids (Tang et al. 2016a; Pelosi et al. 2017b).

In general, thymic NET are more prevalent in men than in women (Phan et al. 2010; Modlin et al. 2017). The only exception to this correlation are SCC, which show no predominance in gender (Ahn et al. 2012; Ströbel et al. 2014).

\subsubsection{Typical Carcinoids}

Typical carcinoids, by definition, have a mitotic rate of $<2$ per $2 \mathrm{~mm}^{2}$, corresponding to about 10 HPF in most modern microscopes, and lack necrosis. They stain strongly with neuroendocrine markers (chromogranin, synaptophysin, neuro-specific enolase, CD56) (Bohnenberger et al. 2017). HE stains display uniform, oval epithelial cells with salt and pepper chromatin and a basophil or eosinophil cytoplasm (Fig. 2). The cells often form trabecular, rosette, festoon, ribbon, solid nest, and glandular growth patterns (Goto et al. 2001; Marx et al. 2015a; Brcic et al. 2016).

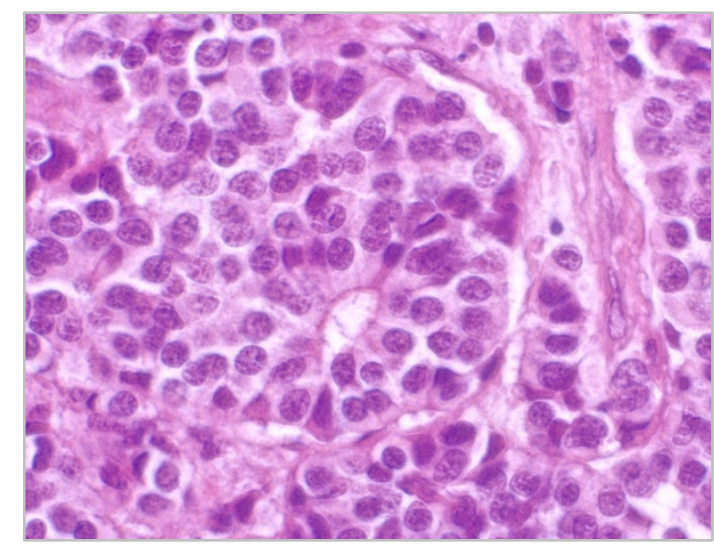

Figure 2: Histomorphology of a typical carcinoid. The cells are round and oval with small bland nuclei. The cells form a rosette. There are typical fine blood vessels surrounding the tumor cells.

Lymph and blood vessel invasion is a common feature. $30 \%$ of patients encounter paraneoplastic phenomena due to hormone production, resulting in i.e. Cushing syndrome, acromegaly, inappropriate production of antidiuretic hormone or ANP, hypercalcemia, and hypophosphatemia (Gal et al. 2001; Ahn et al. 2012; Marx et al. 2015a). These paraneoplastic syndromes may lead to an earlier detection of the tumor. The five-year survival rate of patients with TC ranges from 50 to $100 \%$, depending on the study (Soga et al. 1999; Moran and Suster 2000a). Currently, the ki67 proliferation index is not a required marker for the grading of TC, but it is often used to help differentiate between TC and AC. 


\subsubsection{Atypical Carcinoids}

Atypical carcinoids make up the largest subgroup, accounting for $40-50 \%$ of all thymic NET (Teh 1998; Kulke et al. 2008; Ströbel et al. 2014). This contrasts greatly with AC of the lungs, which only make up $0.2 \%$ of pulmonary NET (Oronsky et al. 2017). Thymic AC differ from TC through a higher mitotic rate, with 2-10 mitoses per $2 \mathrm{~mm}^{2}$ and often, the presence of necrosis (Ahn et al. 2012). Even when mitotic rates are below two, any area of necrosis warrants the diagnosis of an AC. Atypical carcinoids are morphologically and immunohistochemically very similar to TC with strong expression of neuroendocrine markers. However, AC tumor cells often show more polymorphic nuclei and cellular atypia (Fig. 3). Calcifications are also more characteristic for AC and are present in 30\% of tumors (Marx et al. 2015b).

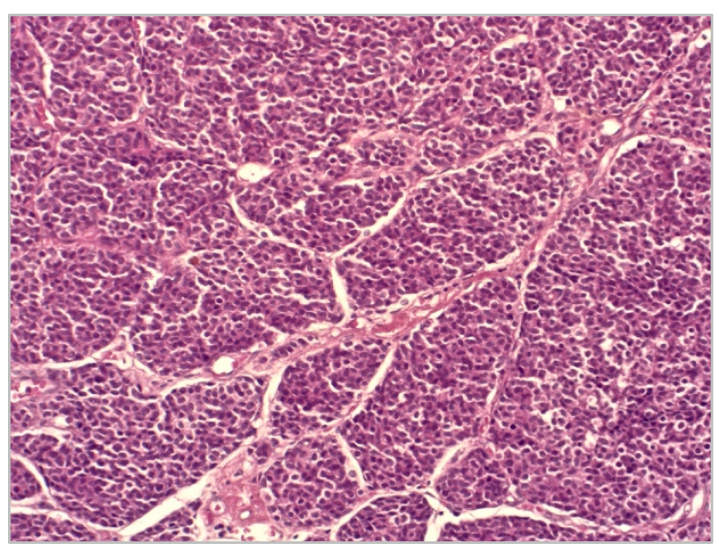

Figure 3: Histomorphology of an atypical carcinoid. Polymorphic nuclei, cellular atypia and calcifications are common features.

$50 \%$ of all AC, when diagnosed, have already metastasized to mediastinal, cervical and supraclavicular lymph nodes or have infiltrated neighboring organs such as pericardium and pleura (Brcic et al. 2016). According to a study by Moran and Suster, the five-year survival rate of $A C$ is $20 \%$, but reaches up to $80 \%$ in other investigations (de Montpreville et al. 1996; Moran and Suster 2000a; Moran and Suster 2000b; Ströbel et al. 2014)

\subsubsection{Large Cell Neuroendocrine Carcinoma}

Large cell neuroendocrine carcinoma are high-grade neuroendocrine tumors with a non-small cell morphology. This means that the diameter of a LCNEC cell should be greater than the diameter of three resting lymphocytes (Travis 2012). By definition, LCNEC exhibit over 10 mitoses per $2 \mathrm{~mm}^{2}$ and often large areas of necrosis (Marx et al. 2015a). The morphologic features common in TC and AC such as trabeculae, rosettes and nesting are less common in LCNEC (Chetty et al. 1997). If present, 
these features are less organized making them difficult to recognize. LCNEC can show a diverse histomorphology due to the definition of LCNEC, which is based on the mitotic rate. Not only can LCNEC display a "low-grade morphology" (i.e. solid or trabecular growth pattern similar to AC, moderate atypia, a mitotic rate at the lower end of the LCNEC spectrum), but this category also encompasses tumors with highgrade morphology in which the mitotic rate is often higher than 20 per 10 HPF (Fig. 4) (Bohnenberger et al. 2017).

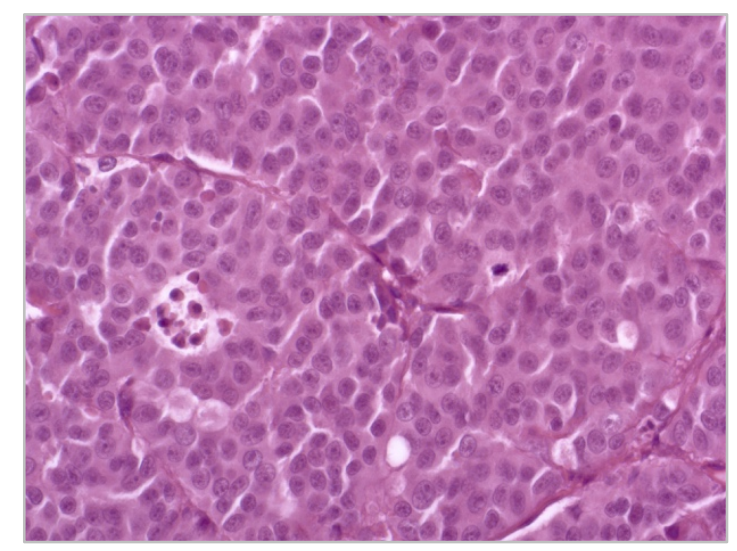

Figure 4: Histomorphology of a large cell neuroendocrine tumor. This LCNEC has features of a well differentiated carcinoid. There are mitoses and apoptosis.

Again, many of these tumors show strong to weak expression of neuroendocrine markers such as synaptophysin, chromogranin, CD56, and keratins (Bohnenberger et al. 2017). When diagnosed, $75 \%$ of LCNEC have already spread to neighboring organs and tissues, or have metastasized to distant organs such as spine, brain and liver (Ferolla et al. 2005; Ahn et al. 2012). Paraneoplastic phenomena are rare. According to different publications, the five-year survival rate of patients with LCNEC ranges from 30 to $66 \%$ (Shoji et al. 2011; Ströbel et al. 2014).

\subsubsection{Small Cell Carcinoma}

Also included in the high-grade neuroendocrine tumor subgroup are small cell carcinoma. These are very rare in comparison to pulmonary SCC, accounting for only $10 \%$ of all thymic NET (Gaur et al. 2010). Formally, SCC have more than 10 mitoses per 2 mm² $^{2}$ and display extensive necrosis (Teh 1998; Gal et al. 2001; Brcic et al. 2016). Often the mitotic rate is much higher, reaching up to 100 mitoses per 10 HPF. Currently, the diagnosis of SCC does not require the expression of neuroendocrine markers, although it is a frequently observed feature (Brcic et al. 2016). The diagnosis rests on morphology on HE stainings. In an HE stained section, tumor cells are often smaller than the diameter of three resting lymphocytes and show scant cytoplasm with 
a large nuclear cytoplasmic ratio, and crush artefacts (Fig. 5) (Gal et al. 2001; Travis 2012). Crush artefacts are due to the fact that the cells are more vulnerable while going into apoptosis or mitosis (Bohnenberger et al. 2017).

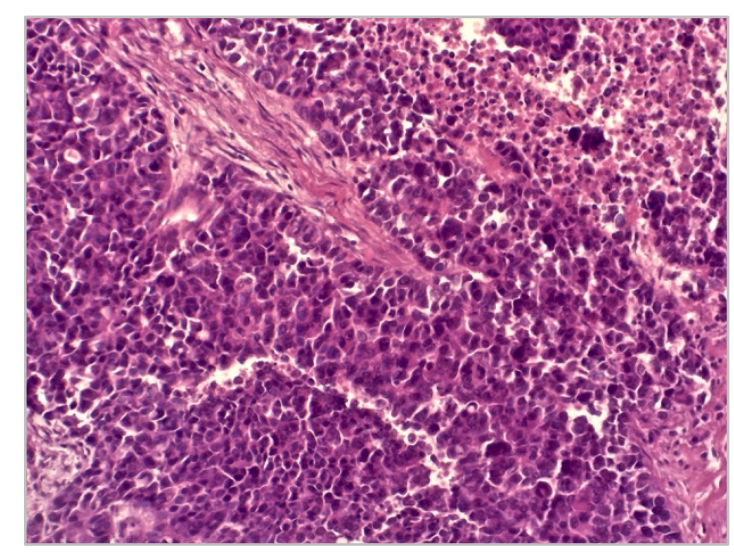

Figure 5: Histomorphology of a small cell carcinoma. This SCC shows typical small cell morphology with crush artefacts and areas of apoptosis.

The nucleoli of tumor cells are often obscured. Patients with SCC often present with local infiltration and distant metastases to bone, brain, liver and lung (Tiffet et al. 2003). This reflects the aggressive nature of these neoplasms. The five-year survival rate has been noted at 0\% (Wick et al. 1982; Ströbel et al. 2014). Recurrences even after tumor resection, are possible.

\subsection{TNET Genetics}

There are very few publications on the genetics of thymic neuroendocrine tumors. However, in the sparse previous studies, in which $\mathrm{CGH}$ was used to determine chromosomal aberrations, it was found that the number of genetic aberrations increase as the tumor grade increases (Ströbel et al. 2014). Typical carcinoids have chromosomal gains on 1, 2q24, 7, 8p, 8q, 9q13, 11q23 and 22 and losses on chromosomes 1p, 3p11, 6q, 10q, and 13q (Pan et al. 2005; Schaefer et al. 2013; Ströbel et al. 2014) . The average chromosomal imbalances found in CGH range from 0-8 for carcinoids (Ströbel et al. 2014). Atypical carcinoids have similar genetic alterations to TC, but their average mutation rate is at the higher end of this spectrum. $A C$ have gains on chromosome 1q, 5p, 5q, 7p, 7q, 8q, 12q24, 17q and 20q, and losses on chromosome 3p, 3q, 4q, 5q, 6q, 10q, 11q and 13q (Pan et al. 2005; Rieker et al. 2005; Ströbel et al. 2014). The most mutations are found in high grade tumors, with average chromosomal imbalances ranging from 0-21 in CGH (Ströbel et al. 2014). Recurrent gains are on chromosomes 1q, 6p, 7, 8q, 12q, and 14 and losses are on chromosomes 3, 4q, and 13q (Pan et al. 2005; Schaefer et al. 2013; Ströbel et al. 
2014). Alterations found only in LCNEC include gains on $2 p, 9 p$ and $17 q$ and losses on chromosome 4p, 8p, 9p, and 18p (Ströbel et al. 2014).

\subsection{WHO Grading of Gastro-Intestinal and Pancreatic NET}

In contrast to the grading system of pulmonary and thymic NET, the grading system of gastro-entero-pancreatic NET utilizes the ki67 index to define subgroups. Ki67 is a protein phosphatase 1 binding protein located in the nucleolus (Booth et al. 2014). It is an important factor in building the perichromosomal compartment, a protein and RNA envelope that coats the chromosomes during mitosis (Booth et al. 2014). Ki67 is expressed in proliferating cells during late $\mathrm{G} 1, \mathrm{~S}, \mathrm{M}$ and $\mathrm{G} 2$ phases of the mitotic cycle (Khan et al. 2013). It is used in pathology to obtain the proliferation index and gain insight into the aggressive potential of a tumor.

NET located throughout the entire gastro-entero-pancreatic system share the same cutoff criteria in the WHO 2015/ENETS grading system (Khan et al. 2013; Nadler et al. 2013). G1 tumors have a mitotic rate $<2$ per $2 \mathrm{~mm}^{2}$ or a ki67 $<3 \%$. G2 tumor mitotic rate ranges from 2-20 mitotic figures with a ki67 from 3-20\%. G3 tumors have $>20$ mitotic figures and a ki67 >20\% (Kim and Hong 2016). Recently, a fourth category was introduced- NET G3 (Basturk et al. 2015). NET G3 exhibit well-differentiated morphology, similar to that of an AC, but show a ki67 labeling index above $20 \%$ (Pelosi et al. 2017b). ATRX, DAXX, P53, and Rb1 are important immunohistochemical markers in discerning NET G3 and NEC in gastro-entero-pancreatic-NET. NET G3 show ATRX/DAXX loss, while NEC show overexpression of p53 and loss of Rb1 (Klöppel 2017). NET G3 may originate from former carcinoids (Tang et al. 2016b).

\subsection{Nomenclature and Grading Controversies}

The nomenclature, classification, and grading of neuroendocrine tumors has been, and still is, an evolving and controversial field. In the previous and third edition of the WHO Classification of Tumors of the Thymus, thymic carcinoids (i.e. TC and AC) are referred to as "well-differentiated carcinoma" (Marx et al. 2015b). This term is misleading because the term "carcinoma" refers to a loss of differentiation. Also in the third edition, LCNEC and SCC are referred to as "poorly-differentiated carcinoma", even though LCNEC and even SCC can show clear differentiation of neuroendocrine features (Marx et al. 2004). Therefore, this nomenclature was replaced by the separation into "low-grade", "intermediate-grade", and "high-grade" tumors in the current WHO fourth edition (Marx et al. 2015a). The current WHO classification system 
is based on morphology, but the inclusion of metric criteria indicates that it is also a grading system (Pelosi et al. 2017a). However, tumor grade often fails to equate to neuroendocrine differentiation, as it was defined in the third edition. Morphology and differentiation remain in disagreement in some cases.

A persisting obstacle in the current classification is that tumor differentiation is not consistently predictive of clinical behavior. Low-grade/well-differentiated tumors may still be biologically aggressive, by metastasizing early and leading to poor prognosis (Klimstra et al. 2010). In contrast, some cases of LCNEC, although showing high proliferations indices, are morphologically low-grade and behave in an indolent fashion (Ahn et al. 2012). This complicates risk stratification among NET.

Not only the nomenclature of TNET, but also the grading criteria has been prone to change. For example, in the pulmonary and thymic neuroendocrine tumor WHO grading system, the cutoff between TC and AC is two mitoses. A grading proposal by Moran et al. defines the cutoff at $\leq 3$ to separate these subgroups (Moran et al. 2009). A proposal by Pelosi et al. integrates mitotic rate, ki67, and necrosis to assign pulmonary NET to three different categories (Pelosi et al. 2017a).

Even within the GEP-NET system, different classification schemes have been proposed. The WHO/ENETS classification relies on set mitoses and/or ki67 index cutoffs and a recent development incorporates a fifth subgroup called "NET G3" (Klöppel 2017). A publication by Hochwald et al. suggests using only mitoses at different cutoffs instead of using the WHO classification (Hochwald et al. 2002). How to best unite nomenclature and grading using histomorphological characteristics to better reflect biological behavior remains to be defined.

\subsection{The Objective of the Study}

Thymic neuroendocrine tumors, regardless of their grade, remain a behaviorally and biologically diverse group. Tumors that appear to be low-grade can mimic high-grade tumor activity by metastasizing and invading surrounding tissue early-on and ultimately leading to dismal prognosis. Further, bland morphological features may disguise the unstable genetic profile of some of these tumors. The opposite phenomenon applies to high-grade carcinoma. These neoplasms may show aggressive and highly proliferative cytological features but remain genetically stable. The correct categorization of each individual TNET is crucial for risk-stratification and for the selection of appropriate treatment and placement of patients into prognostic 
groups. The aim of this study was to gain insight into the most important prognostic factors and grouping variables of TNET. This included determining the histological features, immunohistochemistry expression and chromosomal alterations within TNET to assign these features to TNET subgroups and encompass the prognostic and genetic outliers, or to uncover a new subgroup encompassing these outliers. We hypothesize that the TNET spectrum encompasses another subgroup, similar to the new NET G3 in gastro-entero-pancreatic NET. We further hypothesize that genetics provide stronger insight into the behavior of TNET than morphology.

To test the predictive power of the current WHO classification against other classification schemes, we applied the ki67-based ENETS system and the three-tiered system by Pelosi et al. against a classifier based on chromosomal alterations in TNET. We thus examined the molecular features of a large retrospective series of TNET and adapted the results to different histological classification systems with the goal of establishing robust criteria that allow better stratification of these rare tumors. 


\section{Materials}

\subsection{Tumors and Tissue}

In all, we studied 107 neuroendocrine tumors of the thymus from 102 patients. The formalin-fixed, paraffin-embedded tissue slides and blocks were obtained during surgical resections and diagnostic biopsies collected from 1996 until 2017. 73 of these cases were previously described in a different study (Ströbel et al. 2014). We collected TNET from the University Medical Center Göttingen. Tumors were also contributed from international cooperating centers: Germany (Göttingen and Mannheim), Austria (Graz), Italy (Rome), and the U.S.A. (Silver Spring and Rochester). Inclusion criteria were as follows: a pure neuroendocrine tumor of the thymus, not a combined tumor, enough tumor material to generate at least one HE slide, and strong expression of at least one of the neuroendocrine markers chromogranin A, synaptophysin, NSE, or CD56. According to the WHO 2015 grading scheme, our collection consisted of 22 typical carcinoids, 51 atypical carcinoids, 28 large cell carcinomas, and 6 small cell carcinomas.

In four cases, we were able to study more than one material of a given patient. Case \#1 included a primary tumor and a synchronous metastasis. Case \#2 included a primary tumor and a metachronous metastasis three years later. Case \#3 included two samples of the primary tumor and one metachronous metastasis five years later. Case \#4 included two samples of the primary tumor. All cases were carefully re-reviewed by two observers (HD and PS). The ethics committee of the University Medical Center Göttingen approved the use of human material in this study (ethical approval number Dok_7_2016). 


\subsection{Devices}

\begin{tabular}{|c|c|}
\hline Device & Manufacturer \\
\hline Microtome Leica SM2000R (Ref. No. & Leica Mikrosysteme GmbH, \\
\hline 1464/11.1998, Ser. No.1469) & Wetzlar \\
\hline $\begin{array}{l}\text { pfm Waterbath (Ref. Nr. 041010, Ser. No. } \\
\text { 300359) }\end{array}$ & Pfm medical AG, Köln \\
\hline $\begin{array}{l}\text { pfm cooling plate (Art. Nr. 501003, Ser. No. } \\
\text { 15020001) }\end{array}$ & Pfm medical AG, Köln \\
\hline Tissue-Tek Prisma automated slide stainer & $\begin{array}{l}\text { Sakura Finetek Europe B.V., AJ } \\
\text { Alphen aan den Rijn }\end{array}$ \\
\hline Autostainerlink48 & $\begin{array}{l}\text { Dako, An Agilent Technologies } \\
\text { Company, Hamburg }\end{array}$ \\
\hline IHC advanced staining system & Dako Omnis, Hamburg \\
\hline Zeiss microscope (SIP 51444) Axiophot & $\begin{array}{l}\text { Carl Zeiss Microscopy GmbH, } \\
\text { Jena }\end{array}$ \\
\hline Olympus BX46F microscope & Olympus, Shinjuku, Tokyo \\
\hline Olympus BX53 microscope & Olympus, Shinjuku, Tokyo \\
\hline peqSTAR Thermocycler, Peqlab & $\begin{array}{l}\text { VWR, Avantor, Radnor, } \\
\text { Pennsylvania }\end{array}$ \\
\hline Thermomixer Comfort & Eppendorf AG, Hamburg \\
\hline Qubit fluorometric quantitation & $\begin{array}{l}\text { Thermo Fischer Scientific, } \\
\text { Waltham, Massachusetts }\end{array}$ \\
\hline InnuPure C16 Touch & Analytik Jena AG, Jena \\
\hline $\begin{array}{l}\text { QIAxcel Advanced System, Capillary } \\
\text { Electrophoresis }\end{array}$ & Qiagen, Hilden \\
\hline MiSeq System & Illumina, San Diego, California \\
\hline Centrifuge $5430 \mathrm{R}$ & Eppendorf AG, Hamburg \\
\hline Vortexer Genie 2 & Bender \& Hobein $\mathrm{GmbH}$, Zürich \\
\hline
\end{tabular}

\subsection{Chemicals and Reagents}

$\begin{array}{lll}\begin{array}{l}\text { Chemical/Reagent } \\ \text { Clearify Clearing Agent }\end{array} & \text { Ref.IArt. No. } & \begin{array}{l}\text { Manufacturer } \\ \text { NC9837230 } \\ \text { California }\end{array} \\ \begin{array}{l}\text { EnVision Flex Target } \\ \text { Retrieval Solution, pH low } \\ (50 x)\end{array} & \begin{array}{l}\text { FV805 } \\ \text { DM849, Hamburg, Germany }\end{array} \\ \begin{array}{l}\text { EnVision Flex Target } \\ \begin{array}{l}\text { Retrival Solution, pH high } \\ (50 x)\end{array}\end{array} & \text { DM848 } & \text { Dako, Hamburg, Germany } \\ \text { Wash Buffer } & \text { GC807 } & \text { Dako, Hamburg, Germany } \\ & \text { DM851 } & \end{array}$




\begin{tabular}{|c|c|c|}
\hline Chemical/Reagent & Ref./Art. No. & Manufacturer \\
\hline $\begin{array}{l}\text { EnVision Flex Peroxidase- } \\
\text { Blocking Reagent }\end{array}$ & DM841 & Agilent, Santa Clara, California \\
\hline $\begin{array}{l}\text { EnVision Flex Substrate } \\
\text { Buffer }\end{array}$ & DM843 & Agilent, Santa Clara, California \\
\hline EnVision Flex+ Mouse & GV821 & Agilent, Santa Clara, California \\
\hline Linker SM804 & DM844 & \\
\hline EnVision Flex+ Rabbit & GV809 & Agilent, Santa Clara, California \\
\hline Linker SM805 & DM845 & \\
\hline EnVision Flex/HRP & DM842 & Agilent, Santa Clara, California \\
\hline $\begin{array}{l}\text { EnVision Flex Substrate } \\
\text { Working Solution DAB+ } \\
\text { Chromogen }\end{array}$ & DM847 & Agilent, Santa Clara, California \\
\hline Shandon Eosin $Y$ & 6766010 & $\begin{array}{l}\text { Thermo Scientific, Waltham, } \\
\text { Massachusettes }\end{array}$ \\
\hline Hematoxylin 7211 & $7211 \mathrm{~L}$ & $\begin{array}{l}\text { Thermo Scientific, Waltham, } \\
\text { Massachusettes }\end{array}$ \\
\hline Ethanol $99 \%$ & 2294.5000 & $\begin{array}{l}\text { Chemsolute, Th. Geyer GmbH \& } \\
\text { Co. KG, Renningen, Germany }\end{array}$ \\
\hline Xylol & 371.5000 & $\begin{array}{l}\text { Chemsolute, Th. Geyer GmbH \& } \\
\text { Co. KG, Renningen, Germany }\end{array}$ \\
\hline Ethanol $96 \%$ & 2293.5000 & $\begin{array}{l}\text { Chemsolute, Th. Geyer GmbH \& } \\
\text { Co. KG, Renningen, Germany }\end{array}$ \\
\hline DNAse/RNAse free water & 2352744 & B. Braun, Melsungen \\
\hline PhiX Control v3 & FC-110-3001 & Illumina \\
\hline EB Puffer & 19086 & Qiagen \\
\hline $\begin{array}{l}\text { Milli-Q Direct } 8 \text { Water } \\
\text { Purification System }\end{array}$ & ZR0Q008WW & Merck Millipore, Darmstadt \\
\hline
\end{tabular}

\subsection{Further Materials}

Microscope slides

Süssefrost microscope slides

Voyager Adjustable Pipette

Tacta Pipettes $(10 \mu \mathrm{l}, 100 \mu \mathrm{l}, 1000 \mu \mathrm{l})$

PCR Plates 4ti-0750/TA/IND

Magnet Plate
Thermo Scientific, Braunschweig

Süsse Labortechnik GmbH, Gudensberg Integra Biosciences AG, Zizers, Schweiz Sartorius, Göttingen

4titude Wotton, Surrey 


\subsection{Computer Software and Internet Databases}

$\begin{array}{ll}\text { Description } & \text { Application } \\ \text { Circos 0.69 Software } & \text { Visualization of genomic data } \\ \text { CLC Genomics workbench 11, Qiagen, } & \text { Evaluation of NGS data } \\ \text { Hilden } & \text { Mutation database } \\ \text { ClinVar archives } & \\ \text { https://www.ncbi.nlm.nih.gov/clinvar/ } & \text { COSMIC Catalogue of Somatic Mutations } \\ \text { http://cancer.sanger.ac.uk/cosmic } & \text { Mutation database } \\ \text { MEDOS Web 9.3 Rev. 1424 } & \text { Pathology records } \\ \text { Microsoft Excel 2016 } & \begin{array}{l}\text { Configuration of NET database, } \\ \text { Statistics, construction of diagrams }\end{array} \\ \text { Microsoft Word 2016 } & \text { Thesis writing } \\ \text { http://www.ncbi.nlm.nih.gov } & \text { Pubmed } \\ \text { Statistica 13.3 } & \text { Statistics, construction of graphs } \\ \text { ZEN 2012 (blue edition), version 1.1.2.0, } & \text { Microscope photographs }\end{array}$

Carl Zeiss microscopy GmbH 2011

\subsection{Kits}

Kit

PTLink DM 828

PTLink DM 829

QIAseq Index Kit

GeneRead DNAseq Panel PCR Kit V2

(Human Myeloid Neoplasms Panel)

GeneRead DNAseq Custom Panel V2

(181902 CNGHS-00156X-205)

GeneRead DNAseq Targeted Panel V2

(3027978 NGHA-102X-96)

GeneRead DNA Library I Core Kit 180434

GeneRead DNA Amp Kit 180455

MiSeq Reagent Kit v2 MS-102-2002

innuPREP FFPE DNA Kit- IPC16

Qubit Assay Q32854

AmPure Beads A63881

BIOO BC Adapter and Primer Mix
Manufacturer

Dako, An Agilent Technologies

Company, Hamburg

Dako, An Agilent Technologies

Company, Hamburg

QIAGEN, Hilden

QIAGEN, Hilden

QIAGEN, Hilden

QIAGEN, Hilden

QIAGEN, Hilden

QIAGEN, Hilden

Illumina, San Diego, California

Analytik Jena AG, Jena

Thermo Fischer Scientific

Beckmann Coulter, Brea, California

NOVA-514102 


\subsection{Antibodies}

\begin{tabular}{|c|c|c|c|c|}
\hline Antibody & $\begin{array}{l}\text { Ref. } \\
\text { Number }\end{array}$ & Species & Manufacturer & Concentration \\
\hline Anti-DAXX & HPA008736 & rabbit & $\begin{array}{l}\text { Sigma-Aldrich, St. Louis, } \\
\text { USA }\end{array}$ & $\begin{array}{l}0.1 \mathrm{mg} / \mathrm{ml}, \\
1: 200\end{array}$ \\
\hline Anti-ATRX & HPA001906 & rabbit & $\begin{array}{l}\text { Sigma-Aldrich, St. Louis, } \\
\text { USA }\end{array}$ & $\begin{array}{l}0.2 \mathrm{mg} / \mathrm{ml}, \\
1: 500\end{array}$ \\
\hline Anti-SDHA & HPA041981 & rabbit & $\begin{array}{l}\text { Sigma-Aldrich, St. Louis, } \\
\text { USA }\end{array}$ & $\begin{array}{l}0.3 \mathrm{mg} / \mathrm{ml}, \\
1: 200\end{array}$ \\
\hline Anti-SDHB & HPA002868 & rabbit & $\begin{array}{l}\text { Sigma-Aldrich, St. Louis, } \\
\text { USA }\end{array}$ & $\begin{array}{l}0.2 \mathrm{mg} / \mathrm{ml}, \\
1: 200\end{array}$ \\
\hline Anti-EZH2 & 6034735 & mouse & $\begin{array}{l}\text { Novocastra Leica } \\
\text { Biosystems, Newcastle, } \\
\text { United Kingdom }\end{array}$ & $20 \mathrm{mg} / \mathrm{L}, 1: 50$ \\
\hline Anti- & $9733 S$ & rabbit & Cell Signaling & $1: 500$ \\
\hline H3K27me3 & P68431 & & $\begin{array}{l}\text { Technology, } \\
\text { Massachusetts, USA }\end{array}$ & \\
\hline Anti-gH2AX & $\begin{array}{l}\text { P16104 } \\
\text { 9781T }\end{array}$ & rabbit & $\begin{array}{l}\text { Cell Signaling } \\
\text { Technology, } \\
\text { Massachusetts, USA }\end{array}$ & $1: 100$ \\
\hline Anti-c-myc & Ab32072 & rabbit & Abcam, Cambridge & $\begin{array}{l}0.203 \mathrm{mg} / \mathrm{ml}, \\
1: 100\end{array}$ \\
\hline Anti-Yap1 & $\begin{array}{l}\text { P46937 } \\
14074 S\end{array}$ & rabbit & $\begin{array}{l}\text { Cell Signaling } \\
\text { Technology, } \\
\text { Massachusetts, USA }\end{array}$ & $1: 400$ \\
\hline Anti-Pax 8 & $363 M-18$ & mouse & $\begin{array}{l}\text { Cell Marque, Rocklin, } \\
\text { California }\end{array}$ & \\
\hline Anti-TTF1 & 6041484 & mouse & $\begin{array}{l}\text { Novocastra Leica } \\
\text { Biosystems, Newcastle, } \\
\text { United Kingdom }\end{array}$ & $1: 100$ \\
\hline Anti-CDX2 & GA080 & mouse & $\begin{array}{l}\text { Dako Flex, Carpinteria, } \\
\text { CA }\end{array}$ & RTU \\
\hline Anti-Serotonin & M0758 & mouse & $\begin{array}{l}\text { Dako Agilent Pathology } \\
\text { Solutions, Hamburg }\end{array}$ & $90 \mathrm{mg} / \mathrm{L}, 1: 100$ \\
\hline Anti-SSTRA2 & RBK046-05 & rabbit & $\begin{array}{l}\text { Zytomed Systems, } \\
\text { Berlin }\end{array}$ & $1: 100$ \\
\hline Anti-CD5 & IR082 & mouse & $\begin{array}{l}\text { Dako Flex, Carpinteria, } \\
\text { CA, USA }\end{array}$ & RTU \\
\hline Anti-CD117 & A4502 & rabbit & $\begin{array}{l}\text { Dako Agilent Pathology } \\
\text { Solutions, Hamburg }\end{array}$ & $12.2 \mathrm{~g} / \mathrm{L}, 1: 500$ \\
\hline Anti-Calcitonin & A0576 & rabbit & $\begin{array}{l}\text { Dako Agilent Pathology } \\
\text { Solutions, Hamburg }\end{array}$ & RTU \\
\hline
\end{tabular}




\begin{tabular}{|c|c|c|c|c|}
\hline Antibody & $\begin{array}{l}\text { Ref. } \\
\text { Number }\end{array}$ & Species & Manufacturer & Concentration \\
\hline Anti-Keratin & Z0622 & rabbit & $\begin{array}{l}\text { Dako Agilent Pathology } \\
\text { Solutions, Hamburg }\end{array}$ & RTU \\
\hline $\begin{array}{l}\text { Anti- } \\
\text { Chromogranin } \\
\text { A }\end{array}$ & $238 \mathrm{M}-90$ & mouse & Medac GmbH, Wedel & RTU \\
\hline $\begin{array}{l}\text { Anti-Ki-67 } \\
\text { Clone MIB-1 }\end{array}$ & IR626 & mouse & $\begin{array}{l}\text { Dako Denmark A/S, } \\
\text { Glostrup }\end{array}$ & RTU \\
\hline Anti-RB1 & HPA050082 & rabbit & $\begin{array}{l}\text { Sigma-Aldrich, St. Louis, } \\
\text { USA }\end{array}$ & $\begin{array}{l}0.1 \mathrm{mg} / \mathrm{ml} ; \\
1: 100\end{array}$ \\
\hline Anti-P53 & Clone DO-7 & mouse & $\begin{array}{l}\text { Dako Agilent Pathology } \\
\text { Solutions, Hamburg }\end{array}$ & RTU \\
\hline
\end{tabular}




\section{Methods}

\subsection{Patient Characteristics}

Clinical information regarding age, sex, relevant pre-existing conditions, date of initial NET diagnosis, age at diagnosis and tumor size was obtained from the pathology medical records system Medos. Furthermore, resident registration offices were contacted to obtain information on patients' decease dates, if applicable, for survival statistics.

\subsection{Tumor Tissue Preservation and Preparation}

All tissues were formalin fixed and paraffin embedded (FFPE) archival materials. For $\mathrm{HE}$ and unstained paraffin slides, the FFPE blocks were cut with a microtome into two micrometer sections and were mounted on microscope slides for further analysis.

\subsection{Tissue-Microarray (TMA)}

Tissue-microarray blocks were manufactured by Targos Molecular Pathology $\mathrm{GmbH}$ in Kassel, Germany. First, HE slides of each tumor were viewed under a microscope to determine and mark two tumor hotspots. HE microscope slides and the associated tumor blocks were sent to Targos $\mathrm{GmbH}$. Here, tissue cores were punched out of the donor tumor blocks at the corresponding spots on the marked HE slides. The twomillimeter tissue cores were placed in receptor blocks. Tissue extraction and induction were performed with the TMA Master 3D HISTECH. 60 tumor samples were fit onto one tissue-microarray block. There were two samples for each tumor. The finished TMA blocks were sent back to the University Medical Center Göttingen, where these were mounted on microscope slides.

\subsection{HE Stains}

HE Stains were performed by the HE-staining machine Tissue Tek Prisma, on microscope slides mounted with the tumor tissue. Stains were conducted by the immunohistochemistry lab of the University Medical Center Göttingen. The staining procedure is as follows:

The duration of each incubation lasted two minutes per cycle. If not further specified, each incubation represents one cycle. First, the tumor slides were incubated in Xylol for one cycle, followed by abs. alcohol for two cycles, alcohol $96 \%$ for once cycle, alcohol $75 \%$, distilled water, and hemalum for the three cycles. The tumor slides were then rinsed with warm running water for two minutes and again incubated in eosin $2 \%$ 
for two cycles, distilled water for one cycle and alcohol $96 \%$ for two cycles. The slides are then rinsed with abs. alcohol for two minutes and incubated in xylol for three cycles. Lastly, slides are covered with a film coverslipper.

\subsection{Immunhistochemical Stains}

Immunohistochemical stains were performed on tissue micro array sections on the Dako Omnis advanced staining system and on the Dako Autostainer Link 48. The staining protocol is as follows:

First, the tissue specimens were deparaffinized in clearify clearing agent for one cycle of one minute and rehydrated with DI water for one cycle of five seconds. Then epitopes were retrieved with EnVision Flex Target Retrieval Solution, pH low (TTF1, ki67, DAXX, ATRX, SDHA, Calcitonin, RB1) or high (CDX2, CD5, CD117, chromogranin A, SDHB, EZH2, H3K27me3, $\gamma \mathrm{H} 2 \mathrm{AX}, \mathrm{c}-\mathrm{Myc}$, YAP1, Serotonin, SSTR2A, Keratin, p53), depending on the IHC stain, for 30 minutes. After washing the sections with a wash buffer for two cycles of 2:40 minutes, sections were incubated in the primary antibody for 20 to 30 minutes. Again, the sections were washed with a wash buffer for 10 cycles each consisting of two minutes, then incubated for three minutes in EnVision Flex Peroxidase-Blocking Reagent. Following this, sections were washed with the wash buffer for 10 cycles of two minutes and incubated in EnVision Flex/HRP, marked secondary polymeric antibodies. After washing the slides for two minutes for 20 cycles, washing with distilled water for 31 seconds, and again washing with wash buffer for two minutes and 10 cycles, the slides were finally incubated in EnVision Flex Substrate Working Solution, a substrate chromogen which allows visualization of the immunoprecipitants. Lastly, after another cycle of washing with wash buffer and distilled water, the slides were counterstained with hematoxylin for three minutes and analyzed under a light microscope.

\subsection{IHC Evaluation}

The immunohistochemistry stains were evaluated using a two-tiered and a three-tiered score. Stain intensity and the percentage of positive stained tumor cells were assessed under 10x and 40x microscope objectives. Intensity scores ranged from 0 (no staining) to 1 (weak staining) to 2 (strong staining). The percentage of positive staining tumors cells were scored as $<25 \%,<50 \%$, and $>50 \%$. This resulted in the following evaluation possibilities: $0,1<25,1<50,1>50,2<25,2<50,2>50$. Each tumor received a score for each immunohistochemical stain. For statistical analysis we 
grouped the scores into negative expression $(0,1<25,2<25)$ and positive expression $(1<50,1>50,2<50,2>50)$ or negative expression $(0,1<25)$, weak expression $(2<25$, $1<50)$, and positive expression $(1>50,2<50,2>50)$.

\subsection{Ki67 Labeling Index Evaluation}

The proportion of ki67 positive cells was determined by viewing tumor sections under a light microscope using a 40x objective (field-of-view diameter of $0.55 \mathrm{~mm}$, resulting in $10 \mathrm{HPF}=2.37 \mathrm{~mm}^{2}$ ). Cells were analyzed by eyeballing and estimating the percentage of positive cells. Second, a digital image analysis counter (morphometric analysis) was used, which evaluated the percentage of positive cells with a standard deviation range. Cases in which there was an obvious discrepancy between the morphometric data and an estimate by eyeballing, a photograph of a representative hotspot area was taken at 400 fold magnification, printed out, and positive versus negative tumor cell nuclei were manually counted.

\subsection{WHO, ENETS, and Pelosi et al. Classification}

The HE slides of 72 thymic NET were reviewed for morphologic features and diagnosis under a light microscope. Thymic NET were graded according to the WHO 2015 grading scheme. The cutoffs were: $<2$ mitoses per $2 \mathrm{~mm}^{2}$ and no necrosis for TC; $2-$ $10 / 2 \mathrm{~mm}^{2}$, with or without necrosis for AC; and $>10 / 2 \mathrm{~mm}^{2}$, with or without necrosis for large cell NEC and small cell carcinoma. Mitotic counts were assessed in 10 HPF on HE-stained sections, using an Olympus BX53 microscope (40x objective, field-of-view diameter of $0.55 \mathrm{~mm}$, resulting in $10 \mathrm{HPF}=2.37 \mathrm{~mm}^{2}$ ). Only unequivocal mitoses were counted.

56 of these cases were also classified according to the ENETS WHO classification of gastro-entero-pancreatic neuroendocrine tumors. The same cutoff rates that apply to GEP-NET, were used to grade the TNET: TC $\leq 3 \%$, AC 4-20\%, and HGNEC $>20 \%$. 60 TNET were classified according to the PNET classification proposed by Pelosi et al. This classification is based on the ki67 LI, mitotic count and necrosis. All three parameters are tiered based on three different expression levels, which groups the tumors into G1, G2 and G3 as shown in Table 2 in chapter 4.3.1.

\subsection{DNA Isolation}

Tumor tissue was extracted from the FFPE tumor blocks and centrifuged at maximum speed for one minute. Lysis Solution BC and Proteinase $\mathrm{K}$ were added to the sample and mixed vigorously. The mixture was then incubated at $65^{\circ} \mathrm{C}$ for one hour in a 
thermal mixer. After the lysis step, solution QPS was added to the sample and again centrifuged for one minute. The mixture was incubated in another thermomixer at 90 ${ }^{\circ} \mathrm{C}$ for one hour. MAG Suspension $\mathrm{F}$ and the lysed DNA sample were transferred into the reagent strips of the InnuPure C16. Once the protocol was completed by the InnuPure C16 system, the DNA was extracted in Elution tubes. This process was carried out by the Molecular Pathology Lab of the University Medical Center Göttingen.

\subsection{Next-Generation Sequencing}

NGS was performed as a courtesy of S. Küffer (Institute of Pathology, University Medical Center Göttingen). DNA was isolated from eleven LCNEC following the protocol listed in chapter 3.10. The DNA was quantified using the Qubit Assay (Thermo Fisher) and the concentration of every sample was adjusted to 2,5 $\mathrm{ng} / \mathrm{ml}$. Multiplex PCR was performed using the Human Myeloid Neoplasms Sequencing Panel (Cat. No. NGHS-003) of the GeneRead DNAseq Panel PCR Reagent V2 (Qiagen) according to the manufacturers protocol. DNA amplicons were purified with the AmPure Beads (Qiagen). DNA was end-repaired and adenylated (Aaddition) using the GeneRead DNA Library I Core Kit (Qiagen). The adaptors (Illumina) were ligated using the GeneRead DNA Library Core Kit (Qiagen) followed by a purification step with AmPure Beads. The libraries were then size-selected, and PCR-amplified to increase sequencing depth using the GeneRead DNA Amp Kit (Qiagen). After an additional purification with the AmPure Beads, library sizes and concentrations were measured with QIAxcel capillary electrophoresis (Qiagen). The library was then diluted, pooled and denatured for subsequent sequencing on the MiSeq system using the MiSeq Reagent Kit v2 (Illumina MS-102-2002).

For data analysis, the FastQ files were analyzed in the CLC Biomedical Workbench (Qiagen) using an in-house workflow. The reads were mapped on hg19 (human genome 19, National Center for Biotechnology Information build 37) followed by an initial variant calling. Subsequently, local realignments, primer clipping, and lowfrequency variant calling were performed. False positives were removed based on the read quality and the forward/reverse balance. All variants called were checked manually for sequencing artefacts. The average coverage was $>500$ in all samples, the mutations had at least 50 variant reads. 


\subsection{Shallow Whole Genome Sequencing by Chronix GmbH}

This analysis was performed by Chronix $\mathrm{GmbH}$ (Dr. Julia Beck and Prof. Ekkehard Schütz, Göttingen, Germany). Molecular analyses were possible in 63 tumor samples (13 TC, 30 AC, 16 LCNEC, 4 SCC). Extracted DNA was ultrasonically sheared to an approximate fragment size of 200 bp using a Covaris S2 focused-ultrasonicator. Sequencing libraries were prepared using the NEBNext Ultra II DNA Library Preparation Kit for Illumina (New England Biolabs, Frankfurt, Germany) according to the manufacturer's instructions. Paired-end sequencing ( $37 / 38 \mathrm{bp}$ ) was conducted on an Illumina NextSeq500 with base calling using the bcl2fastq program version 2.17.1.14. An average of $20.2 \mathrm{M}$ (STDEV: $5.5 \mathrm{M}$ ) reads were generated per sample. Sequences were mapped to the human reference genome (HG19) using the BWA version 0.7.12 (average of mapped reads: 15.7 M, STDEV: $6.5 \mathrm{M}$ ) ( $\mathrm{Li}$ and Durbin 2009).

Copy-number analysis based on read-count data was conducted using the QDNAseq $R$ package (version 1.10.0) using a fixed window size of $500 \mathrm{kbp}$ (4407 windows in total) (Scheinin et al. 2014). The obtained log2 ratios were smoothed by applying the circular binary segmentation algorithm using the $\mathrm{R}$ package Copynumber version 1.14.0 (Nilsen et al. 2012). Based on the absolute log2 ratios observed in the four normal samples (mean $+10^{*}$ STDEV) the thresholds for calling copy-number gains/losses in the tumor samples were set to of 0.09/-0.09, respectively.

\subsection{CNI Test by Chronix GmbH}

Library Preparation and Sequencing: Illumina paired-end libraries were generated using DNA extracted from the TNET mentioned above. In brief, fragmented DNA was end-repaired, A-tailed and multiplex sequencing adapters were ligated (NEBNext Ultra II, New England Biolabs). After amplification of adapter-ligated fragments (9-19 cycles) the samples were sequenced on an Illumina NextSeq500 according to manufacturer's protocols.

After de-multiplexing, the sequence data were mapped to HG19. Duplicate reads were removed using the Picard Tools and low-quality reads (mapping quality <60) were removed using Samtools.

Depth of coverage analysis was performed, in which log2 normalized read count ratios were calculated in sliding windows for each tumor, for local reoccurrence and for metastasis. CNV-Seq was used with windows sizes set to either $500 \mathrm{kbp}$ or $5 \mathrm{Mbp}$. 
The significance limits were set to a read count ratio of 0.09 and -0.09 . These values were used to color significant copy number imbalances in the Circos plots using the Circos software.

A chromosomal instability (CNI) score was used for the comparison between samples and between groups of samples (Schutz et al. 2015). In brief, after sequencing, the mapped reads are counted in windows along the chromosomes. A z-score was then calculated for each window. The $\mathrm{CNI}$ score is obtained from all significantly aberrant windows (windows above/below the thresholds) by statistical comparison to a normal population.

Genes, for which causative impacting deletions or amplifications have been described in the COSMIC Cancer Gene Census database, were extracted for regions with gains/losses present in $>10 \%,>20 \%$ and $>25 \%$ in the TC, AC and LCNEC/SCC groups, respectively (Futreal et al. 2004). The percentages of windows above/below the thresholds (copy-number instability, CNI score) were calculated as a general measure of the amount of copy-number aberration present in each tumor.

\subsection{Statistical Analysis}

Statistica version 13.3 (Dell, Germany) was used for statistical analyses. Survival analyses were performed via the Kaplan-Meier method. Statistical significance was tested with the log-rank test. Correlations between metric data were configured with scatterplots and the strength of correlation was assessed with correlation coefficient. To demonstrate the association of grading system subgroups with genetics, boxplots were created. Statistical significance was tested with the Kruskal-Wallis ANOVA test. Associations between potential survival predictors and survival were made with Cox hazard regression ratios. The correlation between immunohistochemistry and tumor subgroups was tested with Mann-Whitney U Test, and Spearman Rank Test. P-values $<0.5$ were regarded as significant. 


\section{Results}

\subsection{TNET Cohort and Clinical Features}

Thymic neuroendocrine tumor cases were gathered from the University Medical Center Göttingen Pathology archives and from international cooperating institutions mentioned in chapter 2. Information regarding patient characteristics was obtained from the Medos database. Survival times were acquired from resident registration offices. The clinical features of the TNET cohort used in this study are summarized in Table 1. Patient characteristics.

Table 1: Patient characteristics.

\begin{tabular}{|l|l|l|l|l|}
\hline & TC & AC & LCNEC & SCC \\
\hline Number of cases & 22 & 51 & 28 & 6 \\
\hline Age median (range)- yrs. & $57(8-78)$ & $54(18-85)$ & $57(16-79)$ & $59(34-74)$ \\
\hline Male sex- no. (\%) & $15(68.2)$ & $42(84.0)$ & $16(57.1)$ & $5(83.3)$ \\
\hline Tumor size median (range)- cm & $11(2.9-12)$ & $8(1-25)$ & $12(6-20)$ & $14(12-15)$ \\
\hline $\begin{array}{l}\text { Systemic therapy regimen- no. } \\
(\%)\end{array}$ & & & & \\
\hline Surgery & $5(22.7)$ & $29(58.0)$ & $6(21.0)$ & $2(33.3)$ \\
\hline Chemotherapy & $1(4.5)$ & $10(20.0)$ & $4(14.3)$ & $2(33.3)$ \\
\hline Radiotherapy & $1(4.5)$ & $17(34.0)$ & $2(7.1)$ & $0(0)$ \\
\hline $\begin{array}{l}\text { Overall survival median follow- } \\
\text { up time (range)- months }\end{array}$ & $48(15-184)$ & $59(2-228)$ & $30(1-181)$ & $1(1-9)$ \\
\hline No. of reported deaths (\%) & $1(4.5)$ & $13(26.0)$ & $8(26.6)$ & $3(50.0)$ \\
\hline
\end{tabular}

With a total of 107 cases, $22(20.5 \%)$ were classified as TC, 51 (47,6\%) as AC, 28 (26\%) as LCNEC and $6(5.6 \%)$ as SCC according to the WHO 2015 classification. There was a male predominance in this cohort: 78 patients were male, 27 patients were female and 2 were unknown. The median age of all patients was 54 at time of diagnosis. Among the 107 cases, 51 tumors (7 TC, 32 AC, 10 LCNEC and 3 SCC) had available survival data. In all, at the time of data collection 25 patients were deceased and 26 were alive. Of 7 TC patients, $6(85 \%)$ were alive and $1(17 \%)$ had died. Of 31 AC patients, 18 (61\%) were alive and 13 (16\%) had died. Of 10 LCNEC patients, $2(20 \%)$ were alive and $8(80 \%)$ had died. Of 3 SCC patients, 0 were alive and $3(100 \%)$ had died. The mean overall survival for all WHO subgroups was 52,2 
months. The median survival for each subgroup was 48 months for TC, 59 months for AC, 30 months for LCNEC and 1 month for SCC. The median tumor size for all groups was $9 \mathrm{~cm}$, with a median tumor size of $11 \mathrm{~cm}$ for TC, $8 \mathrm{~cm}$ for AC, $12 \mathrm{~cm}$ for LCNEC and $12 \mathrm{~cm}$ for SCC. Tumor size did not correlate to diagnosis in this cohort (Fig. 6).

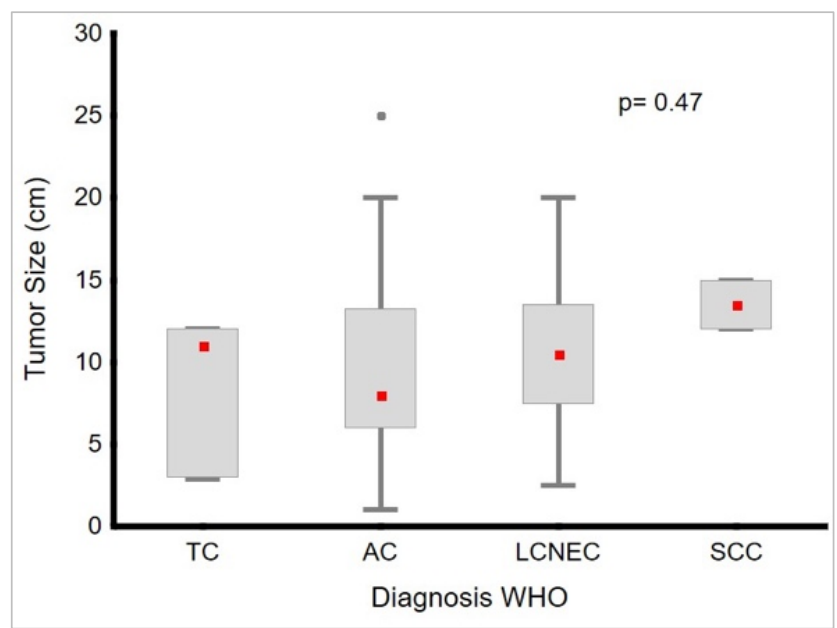

Figure 6: Box plot of tumor size among WHO subgroups. Although the size average increases slightly from AC to $L C N E C$ to SCC, the range of tumor sizes overlaps exceedingly. TC tumor size average is greater than that of $A C$ and LCNEC.

\subsection{Relationship of Proliferation Markers with Overall Survival}

\subsubsection{HE and Ki67 Slide Preparation}

All formalin-fixed and paraffin-embedded tumor blocks were cut with a microtome and mounted on microscope slides. To generate HE slides, the HE-staining machine Tissue Tek Prisma, incubated the tumor-mounted slides according to the protocol listed in chapter 3 . Ki67 is a common proliferation marker expressed in replicating cells during late G1, S, M and G2 phases of the mitotic cycle (Khan et al. 2013). Ki67 stains were conducted on tumor-mounted microscope slides by the Dako Omnis advanced staining system in the immunohistochemistry lab of the UMG. A detailed staining protocol is listed in chapter 3. The HE slides and ki67 slides were reviewed to determine mitotic figures, necrosis, and ki67 labeling index. Mitotic count was assessed by counting all mitotic figures within 10 high-power fields. Ki67 labeling index was assessed by a digital image analysis counter and manually, by eyeballing and estimating the percentage of positive staining tumor cells within a ki67 hotspot. All tumors were classified according to the WHO 2015 grading system together with Prof. Dr. med Philipp Ströbel. 


\subsubsection{Correlation Analysis of Mitoses, Ki67, Necrosis and Survival}

To recognize histological prognostic factors, ki67, mitoses and necrosis were correlated with the overall survival. 18 cases had both ki67 and survival data available. 49 cases had both mitotic count and survival data available. As demonstrated in the scatter plots, mitotic count and ki67 had a weak negative correlation with overall survival $(R=-0,39$, Fig. 7, 8). The higher the mitotic count/ki67, the shorter the survival. However, there were several exceptions to this rule. Some cases with mitoses or ki67 at the lower end of the spectrum corresponded with short survival. The Kaplan-Meier diagram in figure 9, shows that the presence of necrosis corresponds with a worse prognosis. However, there was no statistical significance between necrosis and overall survival in our collection.

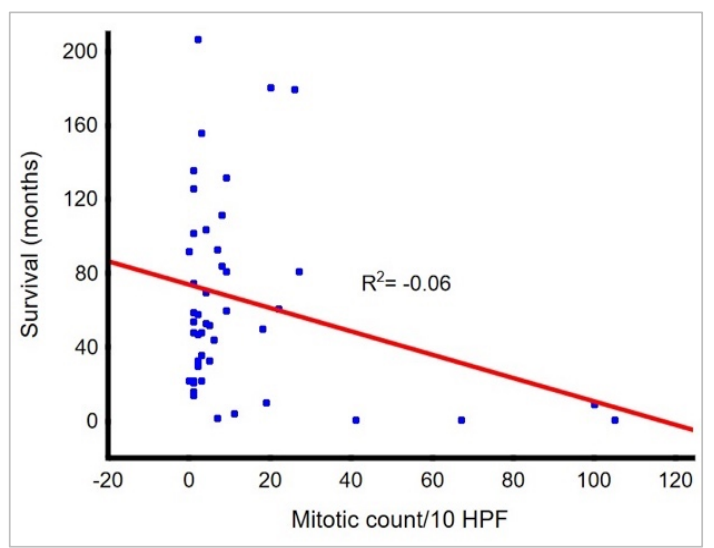

Figure 7: Scatterplot of mitotic count and survival. Low mitotic counts correlate with longer survival, while high mitotic counts correlate with shorter survival. However, exceptions exist. Some cases present with low mitotic counts, yet poor prognosis.

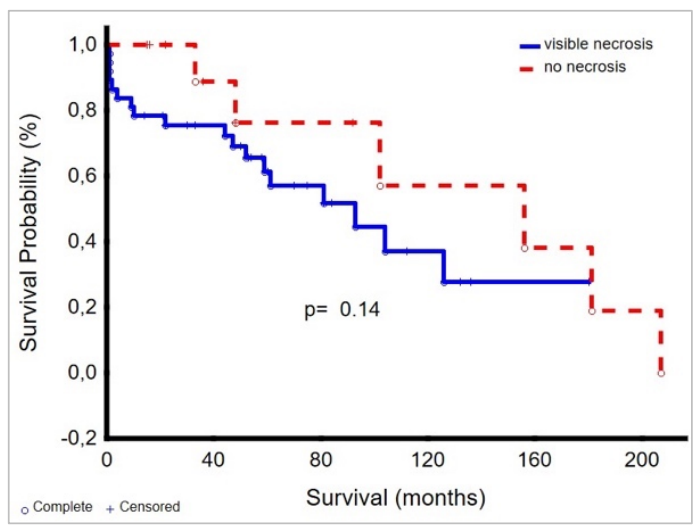

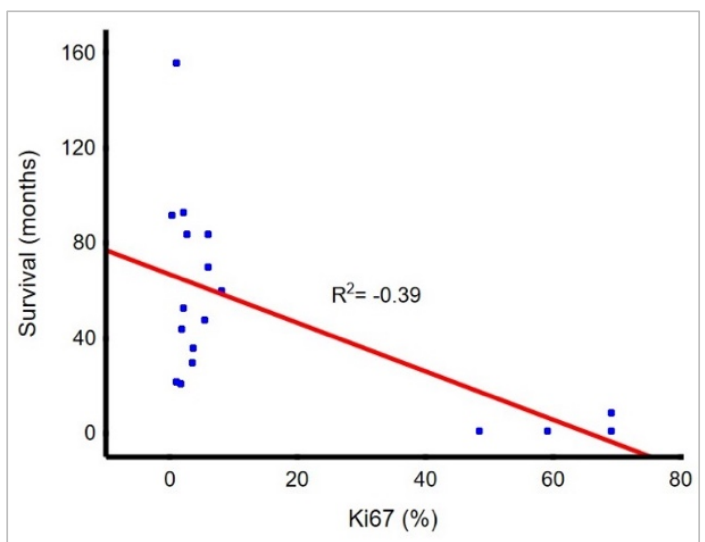

Figure 8: Scatterplot of ki67 and survival. Ki67 at the lower end of the spectrum correlate with longer survival, while ki67 at the upper end of the spectrum correlate with shorter survival. Some cases do not follow this rule.

Figure 9: Survival analysis of necrosis positive and negative tumors. The dashed line representing survival of necrosis-lacking tumors, depicts a steady decrease in survival, but an overall longer survival than the solid line. The solid line represents patient survival of necrosispresenting tumors. This survival curve declines faster than the curve of necrosis lacking tumors. Necrosis is not a statistically significant factor of survival.

\subsubsection{Ki67 of WHO Diagnosis and Correlation with Mitotic Count}

In all, 56 of the 107 TNET had enough tumor tissue available to perform ki67 stains and determine the ki67 labeling index. The cohort included 13 TC, 27 AC, 12 LCNEC 
and 4 SCC according to the WHO 2015 classification. Figure 10 shows the ki67 range of the WHO subgroups. The ki67 range of $A C(0.3-18.8 \%)$ overlapped with the ki67 range of TC (0.1-6.7\%) and LCNEC (16-59\%). The ki67 range of LCNEC showed a very wide spectrum and overlapped with the ki67 of AC and SCC subgroups. The SCC ki67 range was 49-69\%. The median ki67 value for TC was $1 \%$, for AC $4 \%$, for LCNEC 55\% and for SCC 69\% (Average TC: 2, AC: 6, LCNEC: 47, SCC: 62). Thus, the proliferation index rose with rising WHO grade, despite considerable overlap between subgroups. Due to the considerable overlap between WHO subgroups, using the ki67 index to differentiate between WHO subgroups is difficult.

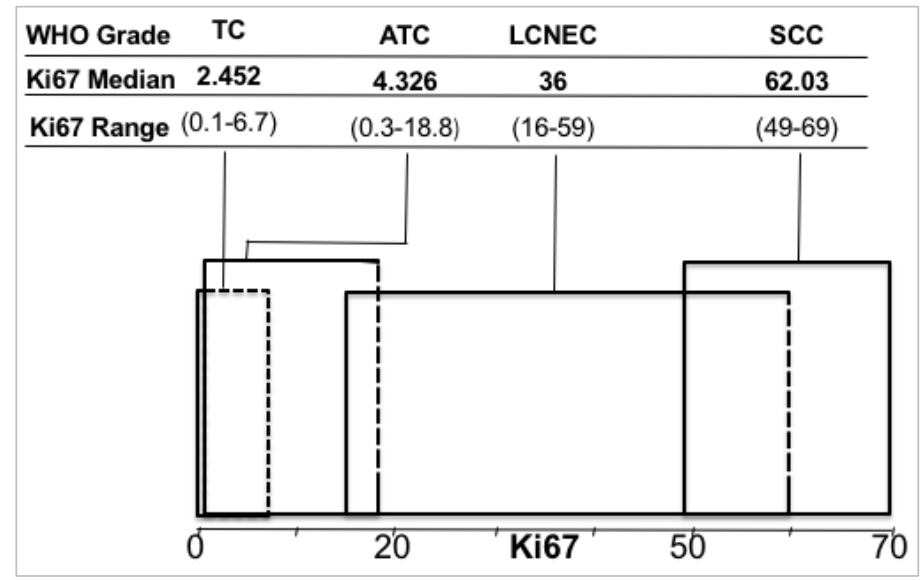

Figure 10: The ki67 spectrum of TNET. The spectrum of the ki67 labeling index increases with rising WHO tumor grade. The spectrum of each tumor grade overlaps with the grade above or below. This makes a distinction between WHO subgroups using the ki67 labeling index difficult.

In the scatterplot in figure 11, mitotic count and ki67 present a positive correlation, with a correlation coefficient $\mathrm{R}^{2}$ of 0.65 . As mitotic count increased so did the ki67 labeling index. However, there are some outliers within the graph. A few TNET exhibited a lower mitotic count around 10, yet still presented with a high ki67 around $70 \%$. The ki67 labeling index did not always correlate with the number of mitoses.

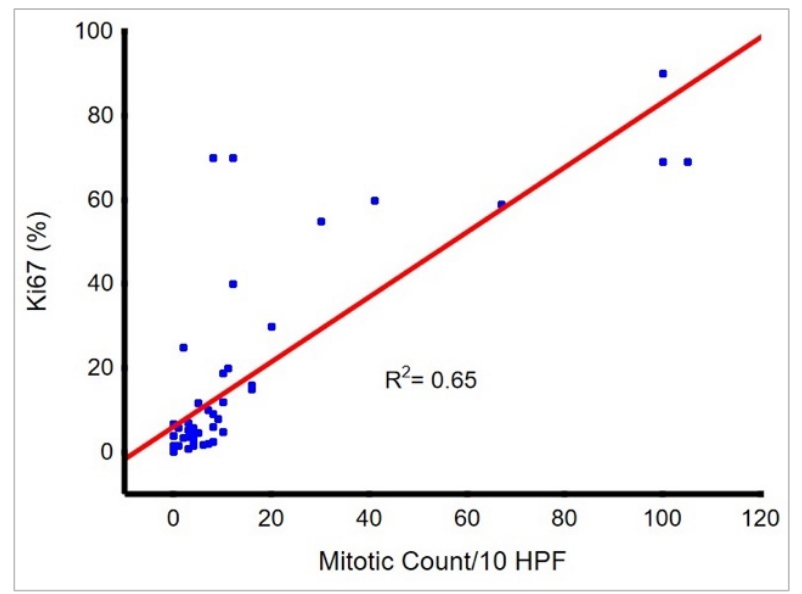

Figure 11: A correlation of mitotic count and ki67. The mitotic count and ki67 show a positive correlation. An increase in mitotic count corresponds with an increase in ki67. Some cases present with lower mitotic counts, but a ki67 at the upper end of the spectrum. 


\subsection{A Comparison of Three Classification Systems}

\subsubsection{The ENETS Classification and Pelosi NET Grading Proposal}

The ki67 labeling index is already a recognized and frequently used marker for grade assignment in the WHO/ENETS classification of gastro-intestinal and pancreatic NET. To determine how the ki67 labeling index influences tumor grade in thymic NET, the ENETS grading system was applied to these tumors. The Pelosi et al. NET classification is a grading proposal to better classify pulmonary NET, but it is not a recommended grading guideline from the $\mathrm{WHO}$. To determine if thymic NET can again share a grading system with pulmonary NET, as is the case in the current WHO classification, the Pelosi et al. pulmonary NET classification proposal was applied to the TNET.

The cutoffs for ki67 in the ENETS grading system are $<3 \%$ for NET G1, 3-20\% for NET G2 and $>20 \%$ for LCNEC/SCC NEC G3. In Pelosi et al., the proposal for the pulmonary NET classification is based on ki67 Ll, mitotic count and necrosis (Pelosi et al. 2017a). The three parameters are tiered based on three different expression levels. The grading system separates NET into NET G1, G2 and G3. At least two out of the three parameters must fall into one level to assign NET to a grade (Pelosi et al. 2017a). Both grading systems are depicted in detail in Table 2.

Table 2: The three grading systems. The WHO, ENETS and Pelosi et al. NET classification were applied to TNET. The WHO classification is based on mitotic count and necrosis and is the standard classification for TNET. The ENETS classification is based on the ki67 labeling index and is a recognized grading system for GEP-NET. The Pelosi et al. NET classification applies both mitoses, necrosis and ki67 at different cutoff values, and is a proposed classification system for pulmonary NET.

\begin{tabular}{|l|l|l|l|l|}
\hline Tumor Grade & $\begin{array}{l}\text { TC/ } \\
\text { NET G1 }\end{array}$ & $\begin{array}{l}\text { ACl } \\
\text { NET G2 }\end{array}$ & $\begin{array}{l}\text { LCNEC/ NEC } \\
\text { G3 }\end{array}$ & $\begin{array}{l}\text { SCC/ } \\
\text { NEC G3 }\end{array}$ \\
\hline $\begin{array}{l}\text { WHO Classification } \\
\text { Mecrosis }\end{array}$ & - & $+/-$ & $+/-$ & $+/-$ \\
Mitoses & $<2$ & $2-10$ & $>10$ & $>10$ \\
\hline $\begin{array}{l}\text { ENETS Classification } \\
\text { Ki67 }\end{array}$ & $<3 \%$ & $3-20 \%$ & $>20 \%$ & $>20 \%$ \\
\hline $\begin{array}{l}\text { Pelosi et al. NET } \\
\text { Classification }\end{array}$ & Level 1 & Level 2 & Level 3 & \\
Necrosis & absent & $\leq 10 \%$ & $\geq 10 \%$ & \\
Mitoses & 2 & $3-47$ & $>47$ & \\
Ki67 (\%) & $<4$ & $4-25$ & $\geq 25$ & \\
\hline
\end{tabular}




\subsubsection{Applying the ENETS and Pelosi et al. Classifications}

By applying the ENETS classification to the 56 tumors (WHO: 13 TC, 27 AC, 12 LCNEC, 4 SCC) mentioned above in chapter 4.2.3, 19 tumors were classified as NET G1, 24 tumors were classified as NET G2, 9 tumors were classified as LCNEC G3 and 4 tumors were classified as SCC G3 (Fig. 12 top). Thus, with the ENETS system, three TC were upgraded to NET G2, nine AC were downgraded to NET G1 and three LCNEC were downgraded to NET G2 (Fig. 12 bottom). Assigning set ki67 values, led to a new grade assignment in 15 of 56 cases, and most often to a downgrading of WHO grade to ENETS grade. This equated to a redistribution of $27 \%$.

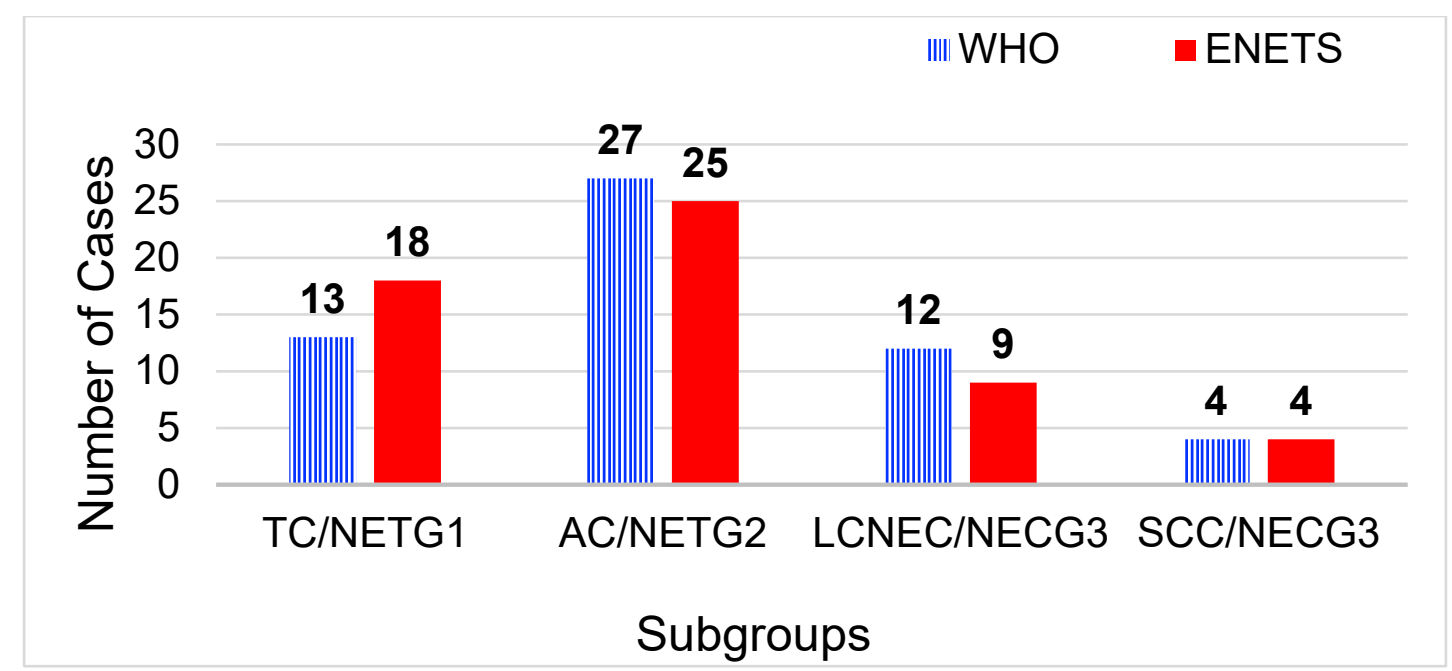

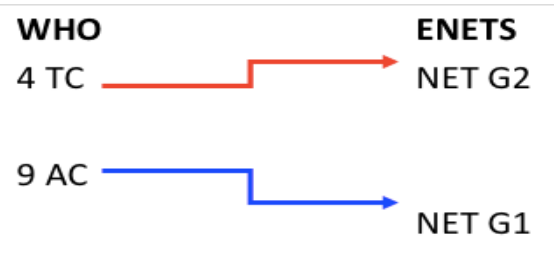

3 LCNEC

The Pelosi et al. grading system was applied to a cohort of 60 TNET, consisting of 20 TC, $30 \mathrm{AC}$ and 10 high-grade NEC. 50 cases had all three parameters available. Nine cases had mitotic count and necrosis values within the same level, and one case had ki67 and necrosis values within the same level, allowing these to be graded. By applying the Pelosi et al. NET classification, nine AC were downgraded to $\mathrm{G} 1$ and five high-grade NEC were downgraded to G2 (Fig. 13 top, bottom). The Pelosi et al. grading proposal led to a redistribution of $14 \mathrm{TNET}$, equivalent to $23 \%$. 

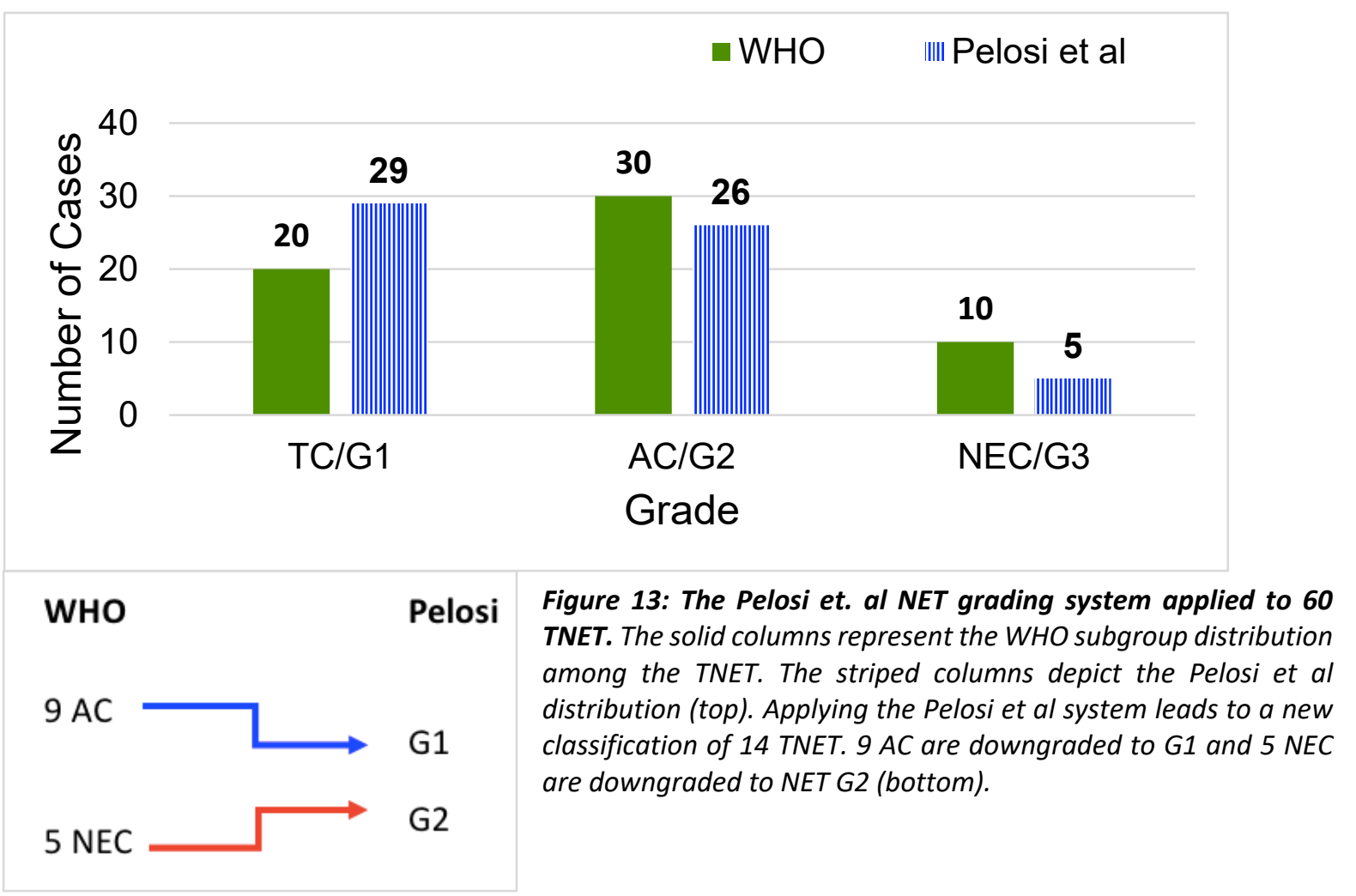

\subsubsection{Survival Analysis of Three Grading Systems}

To uncover how the three grading systems correlate with the survival of TNET patients, survival analyses were configured for each grading system. In the WHO cohort, 52 of 99 cases, in the ENETS cohort, 19 of the 56 cases and in the Pelosi et al. cohort, 25 of the 60 cases had available survival data. In the Kaplan-Meier diagram according to the WHO grading system, TC showed the best overall survival, followed by AC, LCNEC and SCC (Fig. 14). The WHO subgroups provided a clear separation of the four survival curves with minimal overlap between TC and AC subgroups.

The ENETS subgroups followed a similar trend, with NET G1 portraying the best overall survival, followed by NET G2 (Fig. 15). However, the survival curves of NET G1 and NET G2 were very close together and a clear separation between them was not possible. The same applies for the SCC G3 and LCNEC G3 survival curves. No separation between survival probability was possible until the survival curves reached $30 \%$ survival probability. Then, SCC G3 showed longer survival than LCNEC G3. The p-values, calculated with the long-rank test, were 0.0002 for WHO and 0.002 for ENETS, respectively. The WHO grading system better separated subgroups within the carcinoid group, as well as subgroups within the carcinoma group, i.e. TC versus 
AC and LCNEC versus SCC. On the other hand, the ENETS system better discerned between the groups, i.e. carcinoids versus carcinomas.

The survival analysis based on the Pelosi et al. system showed a clear separation of all three groups and was statistically significant in the log-rank test $(p=0.007)$ (Fig. 16). The Pelosi NET G1 group had the best prognosis. The Pelosi NET G3 corresponded with dismal prognosis and seemed to exhibit an identical survival curve to WHO SCC and ENETS SCC G3.

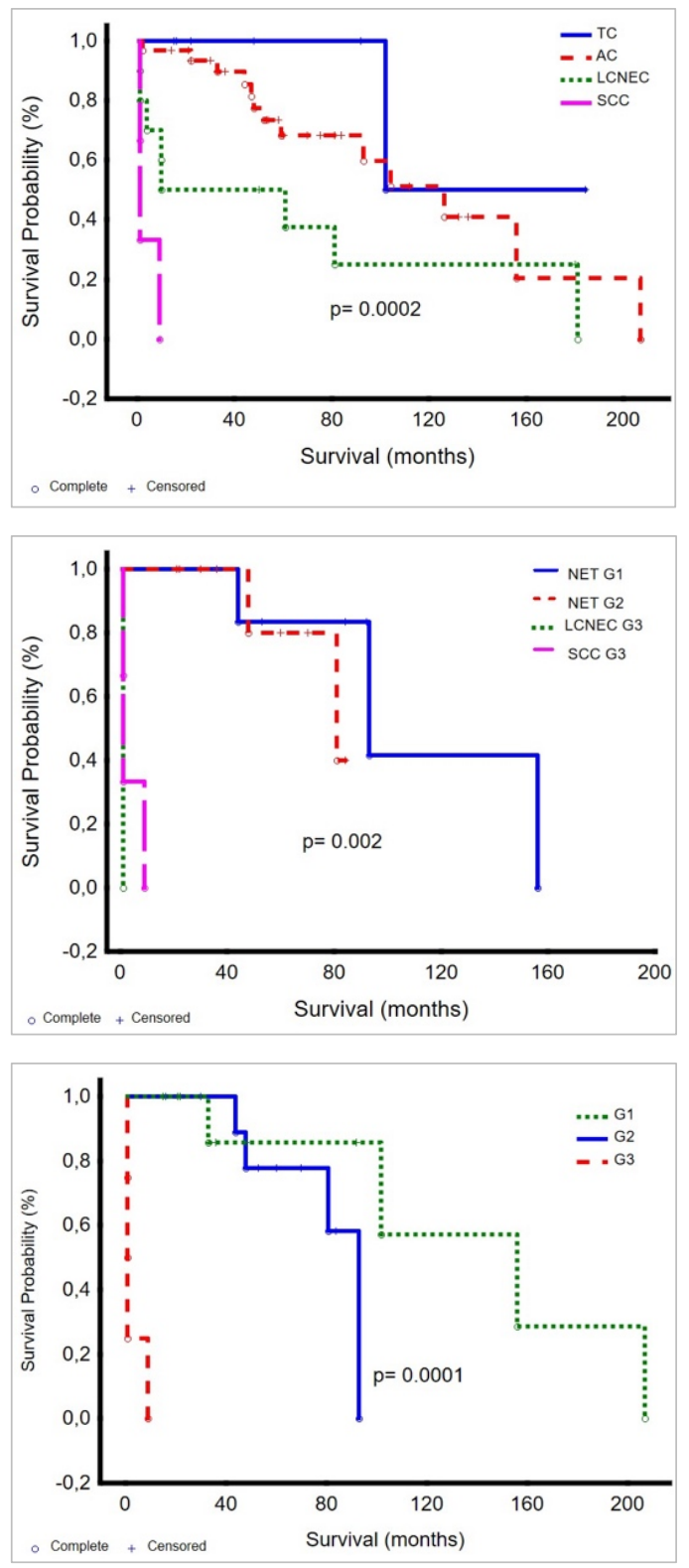

Figure 14: Survival analysis of the WHO subgroups. The solid blue line presents the survival of patients with TC and shows the best overall survival, while the pink long-dashed line presents the survival of patients with SCC and correlates with the shortest survival. Survival times decrease with increasing WHO tumor grade. The graph depicts a strong differentiation of survival between subgroups.

Figure 15: Survival analysis of ENETS subgroups. Survival probability decreases with increasing ENETS grade. There is no clear separation of survival between NET G1 versus NET G2 and no separation of survival between LCNEC G3 and SCC G3. The carcinoid and carcinoma group show a strong divide in prognosis.

Figure 16: Survival analysis of the Pelosi et al. NET classification. The dotted line represents survival of patients with $\mathrm{G} 1$ tumors. These present with the best survival, followed by patients with G2 tumors (solid line). Patients with G3 tumors have the worst prognosis. As Pelosi tumor grade increases the survival decreases.

\subsubsection{Shallow Whole Genome Sequencing and Copy Number Instability Score}

The few publications on genetics in TNET have found chromosomal alterations to increase with WHO tumor grade (Ströbel et al. 2014). To confirm these past correlations, low-coverage whole genome sequencing was performed on the thymic 
neuroendocrine tumors. With this technique, percentages of chromosomal gains and losses were calculated in 61 TNET (13 TC, 29 AC, 15 LCNEC, 4 SCC). Shallow whole genome sequencing was performed by Julia Beck of Chronix Biomedical $\mathrm{GmbH}$.

In the 61 TNET, gains and losses each ranged from $0-41 \%$, respectively. The total of these gains and losses, or total copy number alterations (CNA) ranged from $0.4-81 \%$. The CNA spectrum showed quite an overlap between WHO subgroups and ranged from $16-75 \%$ in TC, $0.4-76 \%$ in AC, $11-81 \%$ in LCNEC, and $60-78 \%$ in SCC. The average for each group was $27 \%$ for TC, $36 \%$ for AC, $52 \%$ for LCNEC and $76 \%$ for SCC.

The circos plots in figure 17 show a compilation of all chromosomal gains and losses, within each WHO subgroup. The overall number of chromosomal aberrations increased with WHO tumor grade. TC had only few large copy number alterations, which included gains on chromosome 1q, 5, 6q, 7q, 8q, 10,11q, 12q, 13q, 18q, 20, $21 q$ and 22q, and losses on chromosome 1, 2p, 4p, 8, 10p, 11p, 15q, 17p, 18p and 22q. There were only a minimal number of overlapping alterations in TC and AC including gains on chromosome 1q, 7q, 10, 12q, 21 and 22, and losses on chromosome $1 \mathrm{p}, 2 \mathrm{p}, 4 \mathrm{p}, 10 \mathrm{p}, 11 \mathrm{p}$ and $17 \mathrm{p}$. In contrast, $\mathrm{AC}$ and high-grade tumors showed extensive overlap. Alterations exclusive only to high-grade tumors included gains on chromosome 1p, 3p, 11q, 17q and losses on chromosome 1q, 7p, 8q, 14q, $15 p, 15 q$. The heatmap in figure 18 and the circos plots show that the chromosomal region $13 p$ is not modified in our TNET cohort, nor are any hotspots identifiable (Fig. 18). 


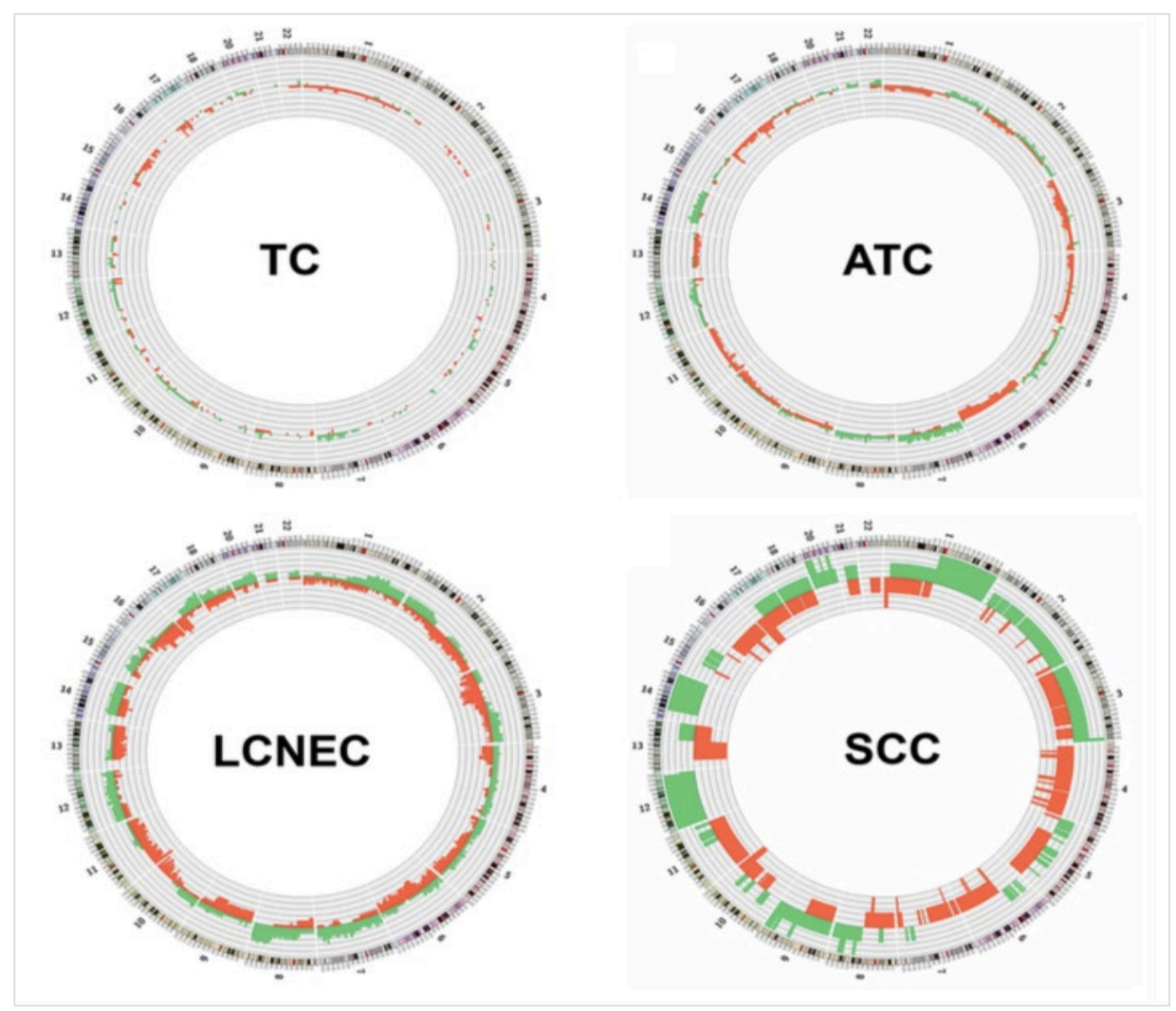

Figure 17: The circos plots depict a compilation of all chromosomal gains and losses among the WHO subgroups. TC present with the least amount of chromosomal aberrations. As the WHO tumor grades increases, so does the chromosomal aneuploidy. SCC demonstrate the most gains and losses.

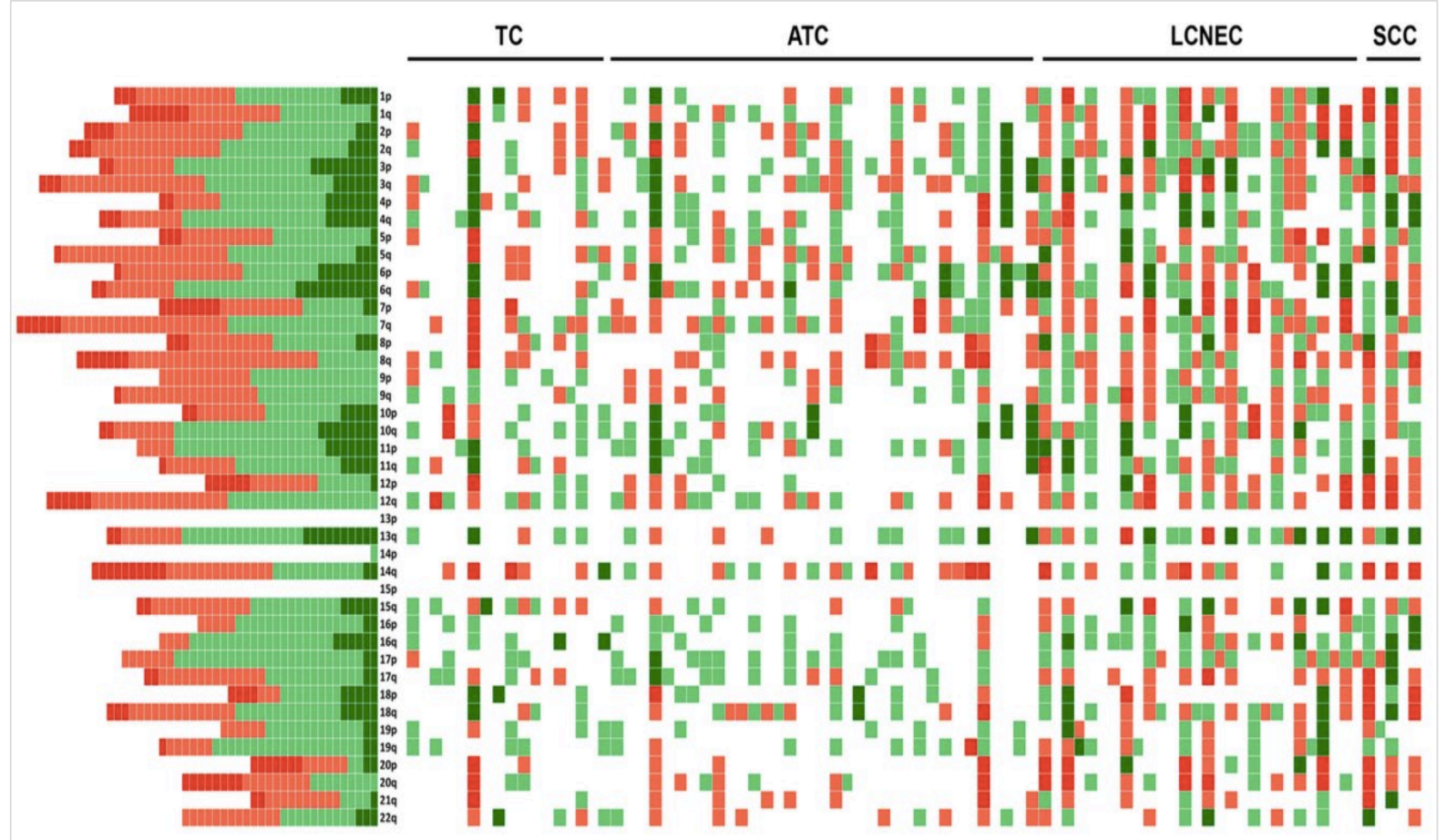

Figure 18: The heatmap shows the chromosomal gains and losses for each chromosome arm amongst the WHO subgroups. The chromosomal aneuploidy increases with WHO tumor grade. Chromosomal regions 13p and $15 p$ do not show any alterations, while chromosome $7 p$ has the most alterations in all. 
We also determined the copy number instability score $(\mathrm{CNI})$ of 63 TNET. The CNI score represents the overall genomic instability e.g. amplifications and losses of genetic material within tumor cells. Of the 63 TNET, 13 were TC, 29 were AC, 17 were LCNEC and four were SCC. CNI scores ranged from 0.45-19 for TC, 0-97.69 for AC, 3.12-83.44 for LCNEC, and 63.14-80.71 for SCC. The averages were 15 for TC, 17 for AC, 47 for LCNEC and 70 for SCC. Interestingly, there were three extreme outliers: TC with a score of $94.4 \%$, AC with a score of $97.7 \%$ and LCNEC with a score of $3.1 \%$. In all, a positive correlation was observed; high tumor grade associated with a higher $\mathrm{CNI}$ score. But there was a large overlap between subgroups. Figure 19 shows the positive correlation between WHO grade and CNI.
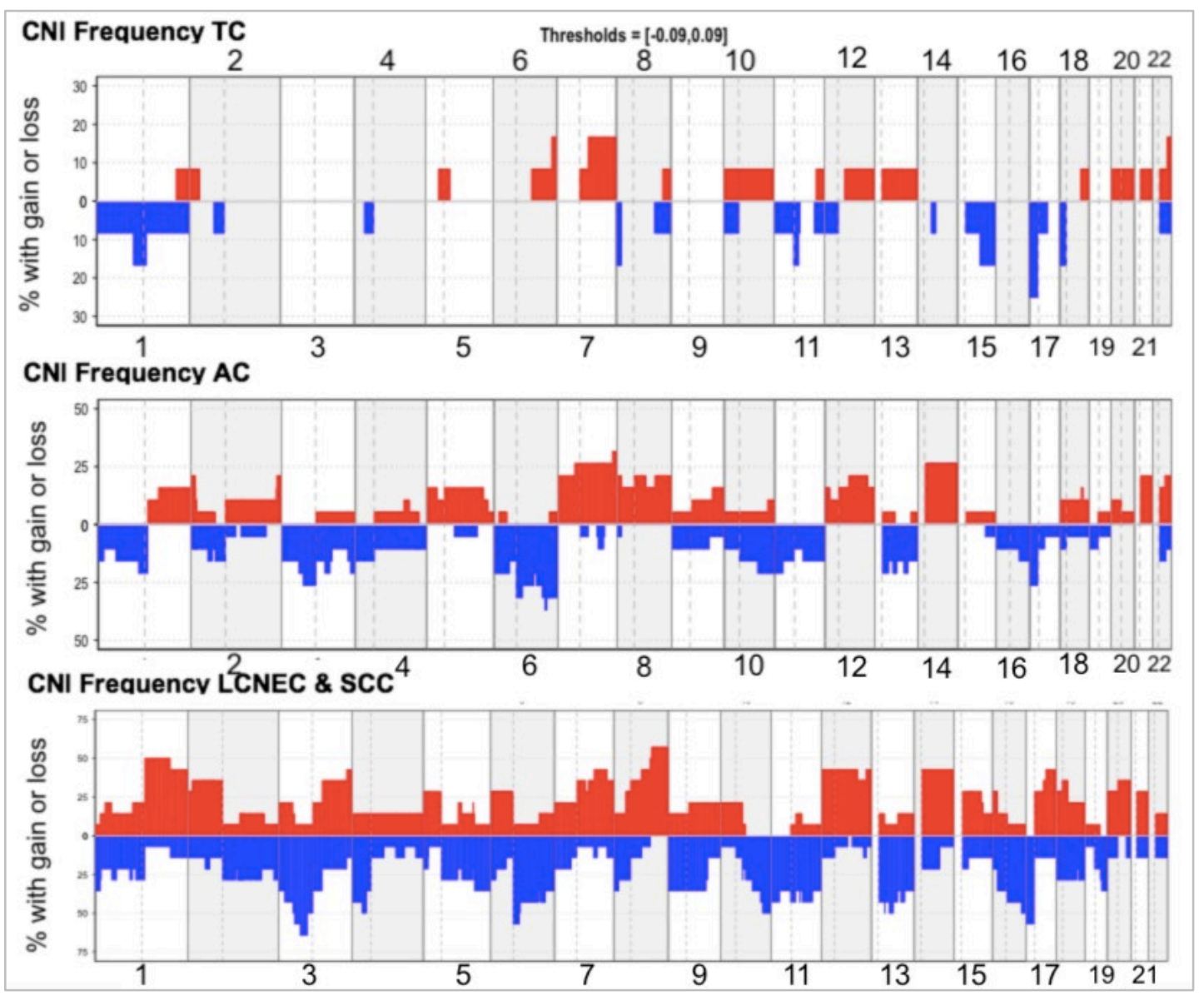

Figure 19: CNI frequency increases with WHO tumor grade. The CNI score, which is calculated from NGS results, corresponds positively with WHO tumor grade. The higher the grade, the greater the chromosomal number instability score

\subsubsection{Three Grading Systems Correlated with Genetic Aberrations}

Boxplots were constructed to correlate between WHO/ENETS/Pelosi et al. tumor grade and chromosomal aberrations. In figures 20, 21 and 22 the shallow whole genome sequencing results are grouped according to the three classification systems. 
These demonstrate a similar trend. Chromosomal aberrations increased with tumor grade. The boxplot based on the WHO grading system, displays a gradual increase in aberration average with rising tumor grade. There was a good discernment between all four subgroups. This finding was statistically significant in the Kruskal-Wallis ANOVA test $(p=0.0036)$.

The ENETS grading system showed strong differentiation of CNA between the lowgrade tumors (NET G1, NET G2) and high-grade carcinoma (NEC G3), but no differentiation within these groups. The difference between low- and high-grade groups was confirmed with a significant Kruskal-Wallis ANOVA test $(p=0.0005)$.

When correlating Pelosi et al. grade to the total chromosomal aberrations, a positive trend was observed, with the most distinct differentiation between G2 and G3. The separation of subgroups based on the Pelosi classification was also statistically significant (Kruskal-Wallis ANOVA $p=0.007$ ).

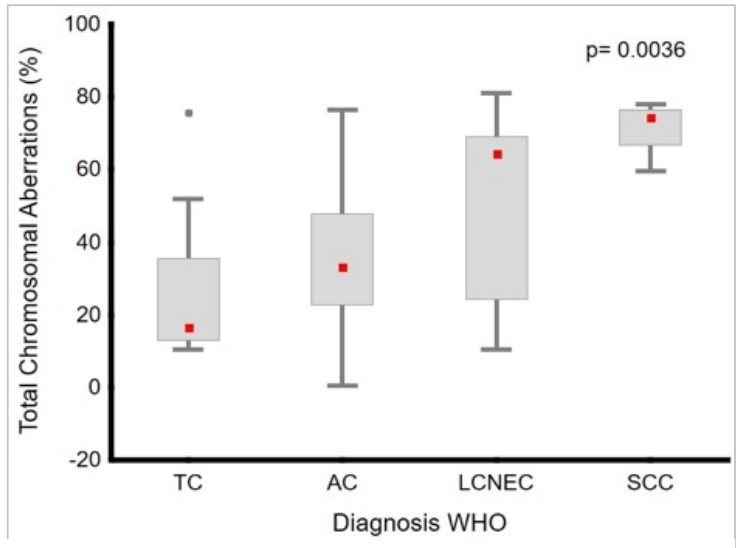

Figure 20: A correlation of WHO tumor grade with chromosomal aberrations. As the WHO tumor grade increases, the total chromosomal aberrations increase as well. TC present with the least aberrations, while SCC present with the most.

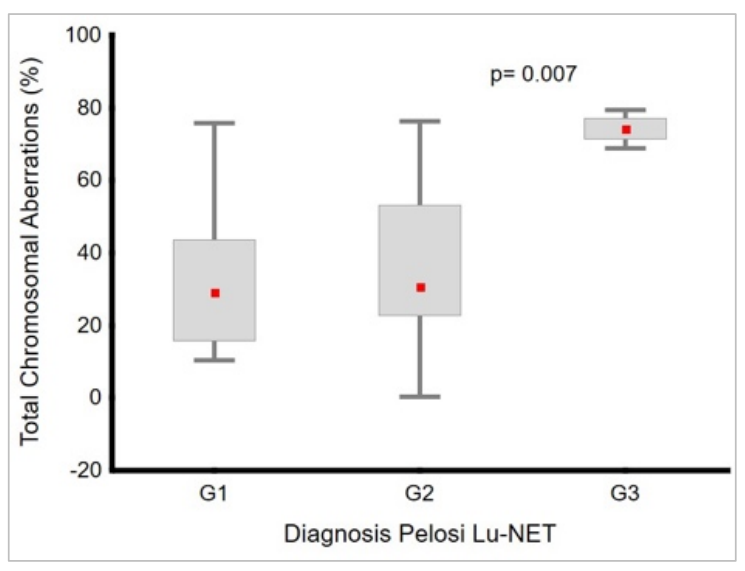

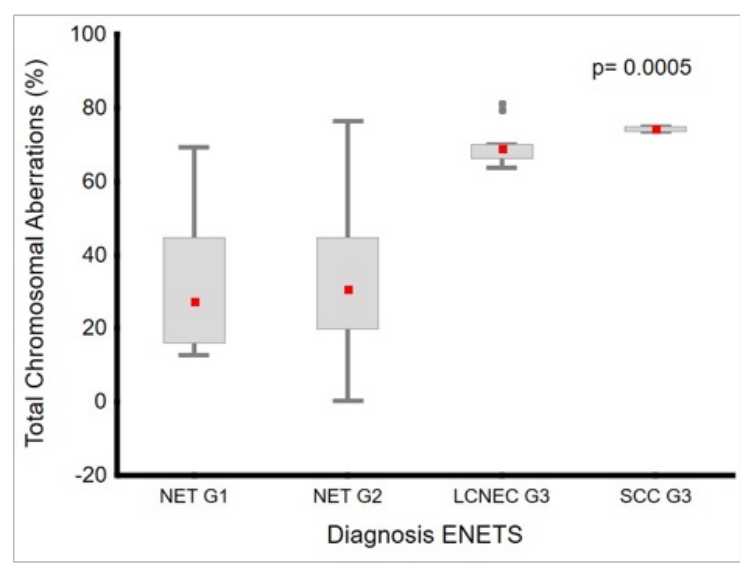

Figure 21: A correlation of ENETS tumor grade with chromosomal aberrations. As the ENETS tumor grade increases, the total chromosomal aberrations increase as well. ENETS does not separate chromosomal aberrations within the NET G1/G2 groups or within the LCNEC G3/SCC G3 groups.

Figure 22: A correlation of Pelosi et al. NET tumor grade with chromosomal aberrations. As Pelosi et al tumor grade increases, the total chromosomal aberrations increase as well. The Pelosi et al grading system does not differentiate chromosomal aberrations between $\mathrm{G} 1$ and $\mathrm{G} 2$. 
Figures 23,24 , and 25 , correlating $\mathrm{CNI}$ with the three grading systems, reinforces the data found amongst the previous three boxplots. The $\mathrm{CNI}$ averages among WHO subgroups showed a gradual average increase with WHO tumor grade, but LCNEC showed a wider range of values. Again, the ENETS classification allowed for a strong separation between low-grade and high-grade subgroups, but no separation within low- and high-grade groups. In figure 25, the boxplot illustrates a positive correlation between $\mathrm{CNI}$ score and Pelosi et al. NET grade. However, the boxplot shows quite an overlap of $\mathrm{G} 1$ and $\mathrm{G} 2 \mathrm{CNI}$, and strong separation of $\mathrm{G} 2$ and $\mathrm{G} 3 \mathrm{CNI}$. The findings of ENETS and Pelosi et al. boxplots were statistically significant $(p=0.0013, p=0.0149)$.

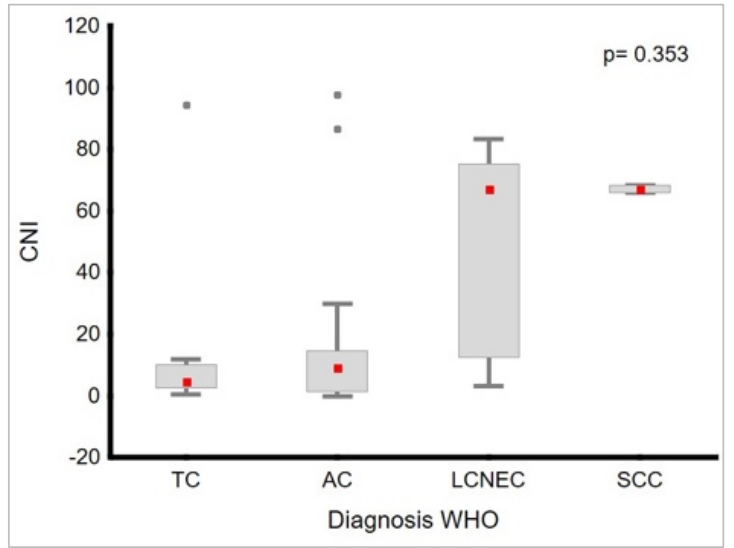

Figure 23: A correlation of WHO tumor grade and CNI. The CNI score increases with rising WHO tumor grade. The WHO classification does not discern the CNI averages between $T C$ and $A C$, nor between LCNEC and SCC.

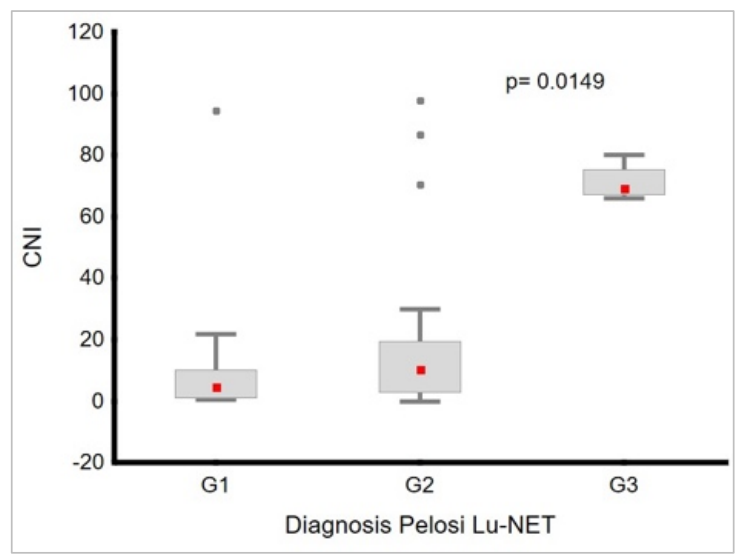

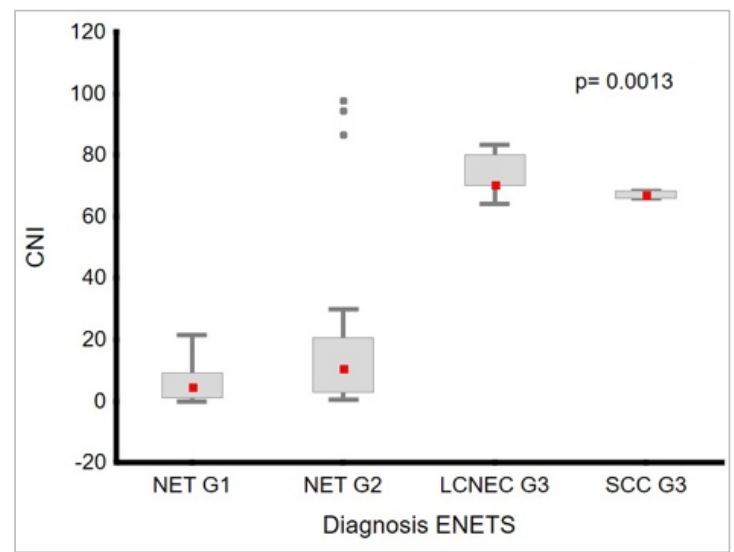

Figure 24: A correlation of ENETS tumor grade and CNI. CNI score increases with ENETS grade, but CNI scores hardly differ among NET G1 and G2, or LCNEC G3 and SCC G3.

Figure 25: A correlation of Pelosi et al. NET tumor grade and CNI. The CNI score increases with Pelosi NET grade. The Pelosi classification does not discern CNI scores of G1 and G2 groups.

\subsubsection{Genetic Alterations Compared to Mitotic Count, Ki67, and Survival}

The scatterplots in figures $26-29$ correlate total chromosomal aberrations and CNI with mitoses and ki67. The two markers representing chromosomal copy number variations displayed a weak, but positive correlation with mitotic count and ki67 labeling index. CNI showed the strongest correlation with ki67 $\left(R^{2}=0.45\right)(F i g .29)$. It 
is apparent from the scatterplots that mitotic count and ki67 do not always associate with genetic variations. Figures 26 and 27, demonstrate that tumors with ki67 or mitoses at the lower end of the spectrum, can still be accompanied by very high chromosomal number aberrations.

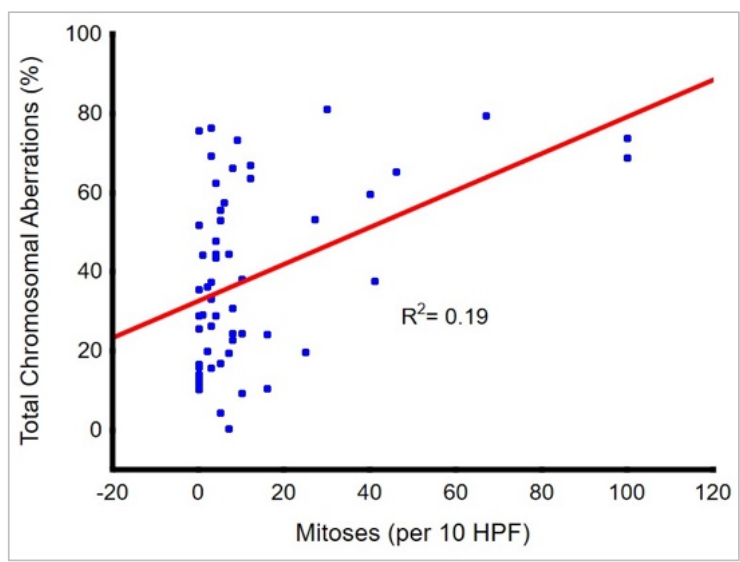

Figure 26: Mitotic count and total chromosomal aberrations show a weak positive correlation. Some cases demonstrate a low mitotic count but are still highly genetically aberrant.

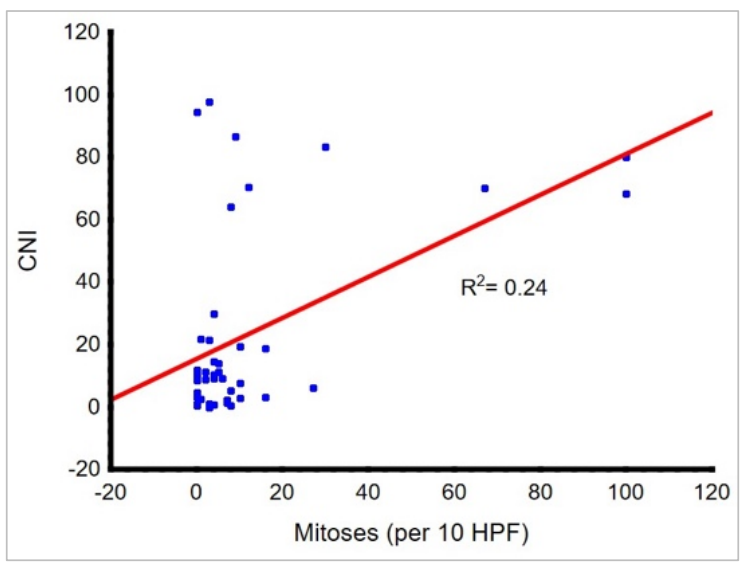

Figure 28: Mitotic count and CNI show a weak positive correlation. Some cases exhibit a low mitotic count but are still highly genetically aberrant.

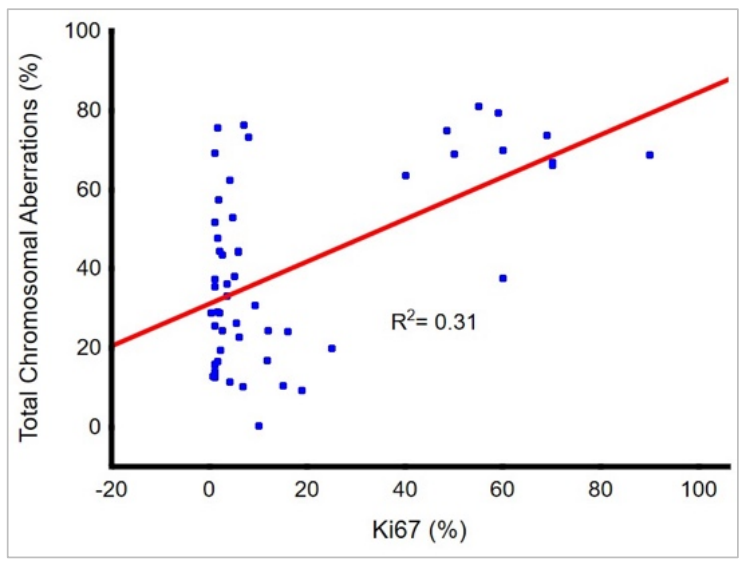

Figure 27: Ki67 and total chromosomal aberrations show a weak positive correlation. Some cases demonstrate a low ki67 but are still highly genetically aberrant.

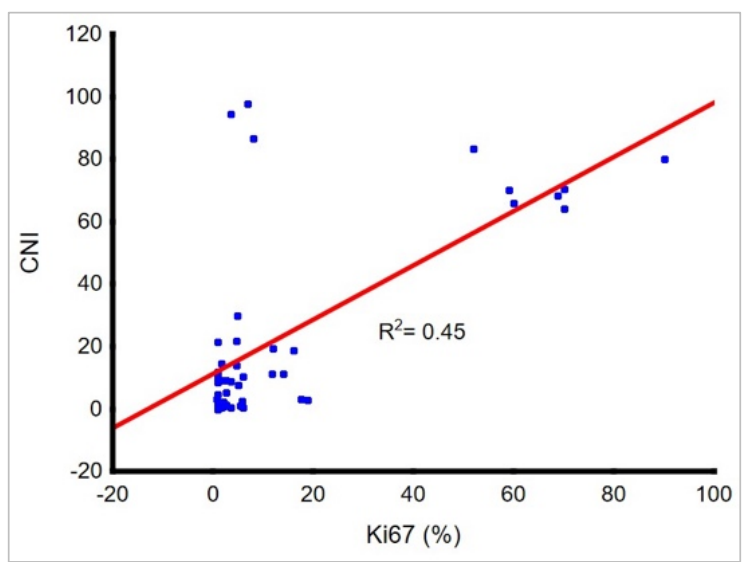

Figure 29: Ki67 and CNI show a positive correlation. The higher the ki67 labeling index, the higher the CNI.

When correlating genetic alterations with overall survival, the total chromosomal aberrations and CNI presented a weak, negative correlation to survival (Fig. 30-31). The higher the genetic variations, the lower the overall survival. The strongest correlate to survival was total chromosomal aberrations $\left(R^{2}=-0.35\right)$ (Fig. 30). Even so, both scatterplots expose cases with genetic number aberrations at the lower end of the spectrum, but still present worse prognosis than cases with higher chromosomal number aberrations. 


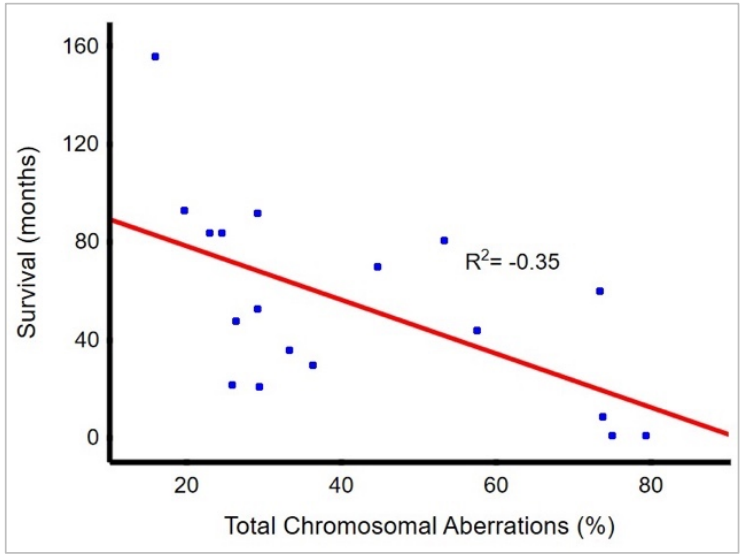

Figure 30: Total chromosomal aberrations and survival show a weak negative correlation.

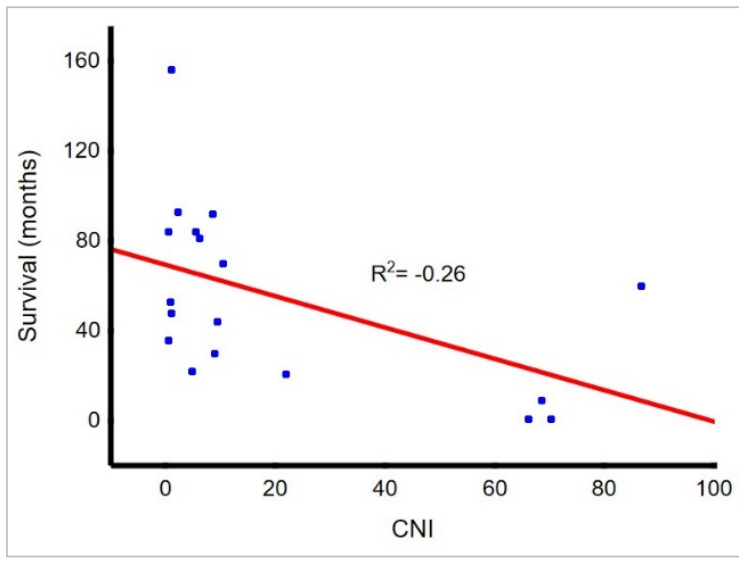

Figure 31: CNI and survival show a weak negative correlation.

\subsection{Clustering Analyses of Shallow Whole Genome Sequencing Data}

We carried out a principle component analysis (PCA). This PCA was based on the shallow whole genome sequencing data $(n=61$ TNET), and establishes which tumors fall into similar genetically altered groups, despite WHO tumor grade (Fig. 32). Two main clusters formed from this analysis. The "carcinoid cluster", on the left side of the diagram, was made up of mostly TC and AC tumors. The "high-grade cluster" was composed of mainly LCNEC and SCC. Interestingly, both clusters exposed outliers. The carcinoid cluster included three LCNEC, while the high-grade cluster enclosed four AC and even one TC. The LCNEC outliers in the carcinoid cluster showed chromosomal variants similar to those found in carcinoid tumors. AC and TC outliers in the high-grade cluster resembled chromosomal variants more common in NEC. This again shows that the number of genetic variations is not always WHO subgroup specific. 


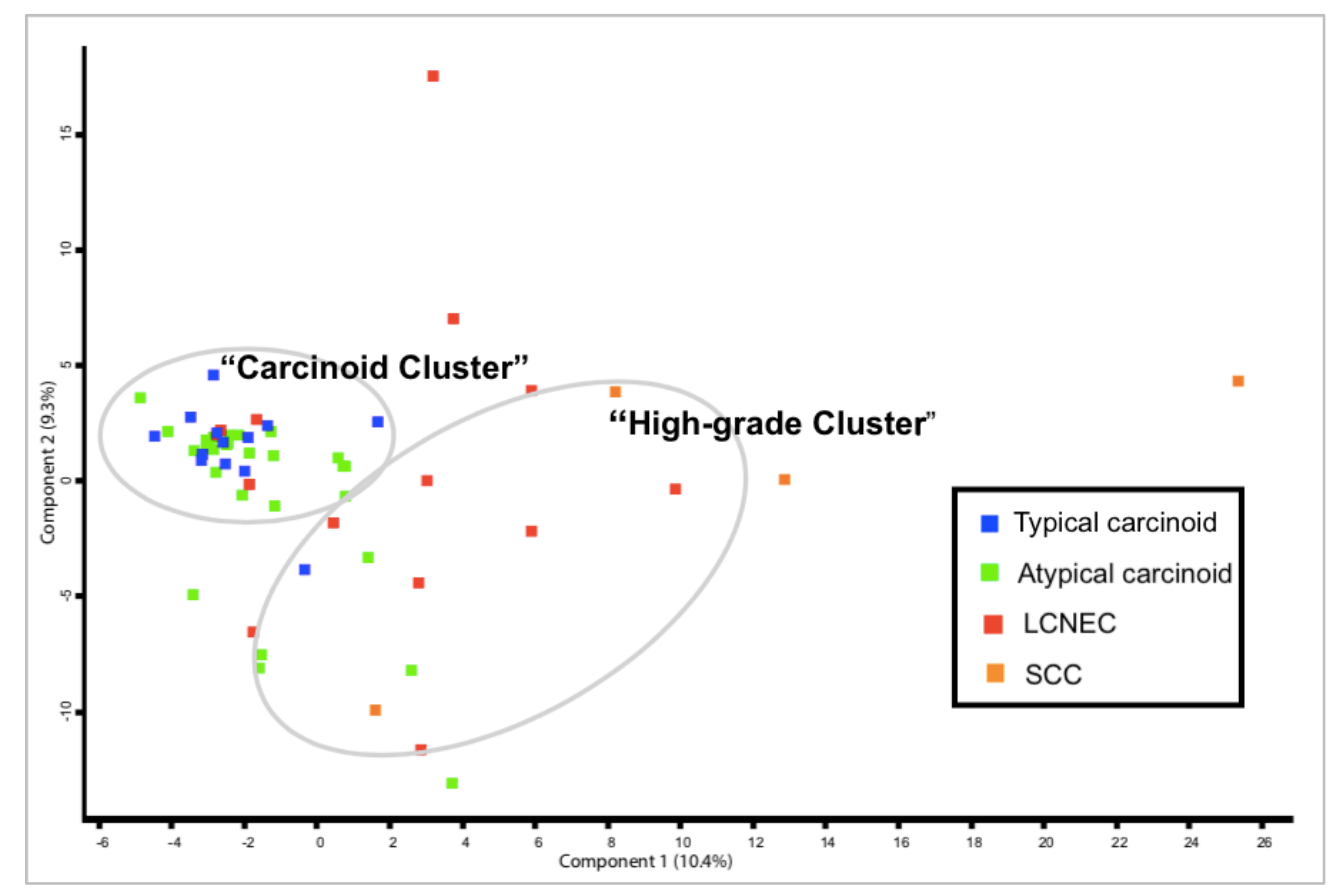

Figure 32: Two genetically distinct clusters form in the PCA analysis. The carcinoid cluster contains mostly TC and AC and a few LCNEC. The high-grade cluster contains mostly LCNEC and SCC, but also a few AC and one TC. Each cluster contains grade outliers that are nonetheless genetically similar.

The two clusters created statistically significant survival curves in the Kaplan-Meier diagram. The high-grade cluster tumors presented with a worse outcome than the carcinoid cluster tumors (Fig. 33). Thus, more chromosomal aberrations lead to a worse outcome, independent of tumor grade.

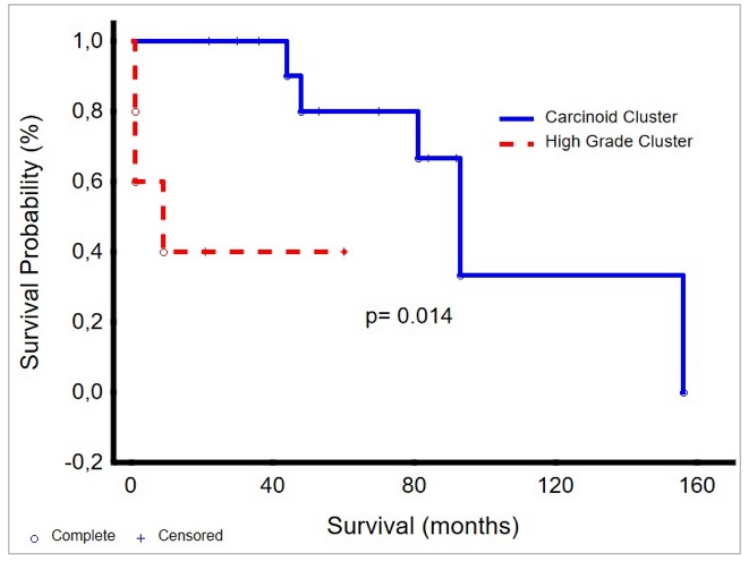

Figure 33: The two genetically distinct clusters form prognostically distinguishable groups. The carcinoid cluster, including the LCNEC within the cluster, exhibit longer overall survival than the high-grade cluster. The high-grade cluster presents with shorter survival times even though TC and AC tumors are included in this group.

Unsupervised clustering of CNIs resulted in three major clusters (Fig. 34). Cluster 3 (CNIhigh) showed the highest degree of CNA and contained all four SCC and nine LCNEC, but also three AC. Cluster 2 (CNlintermediate) with intermediate CNA frequency and cluster 3 with low CNA frequency contained AC, TC, but also LCNEC cases. Morphology and proliferation indices (i.e. tumor grade) were not able to predict the $\mathrm{CNI}$ clusters, as these indices showed significant overlap between clusters. The WHO 
classification failed to correctly assign 7 TC (6 intermediate, 1 high), 19 AC (14 low, 5 high) and 7 LCNEC (2 low, 5 intermediate) to the expected equivalent CNI group.

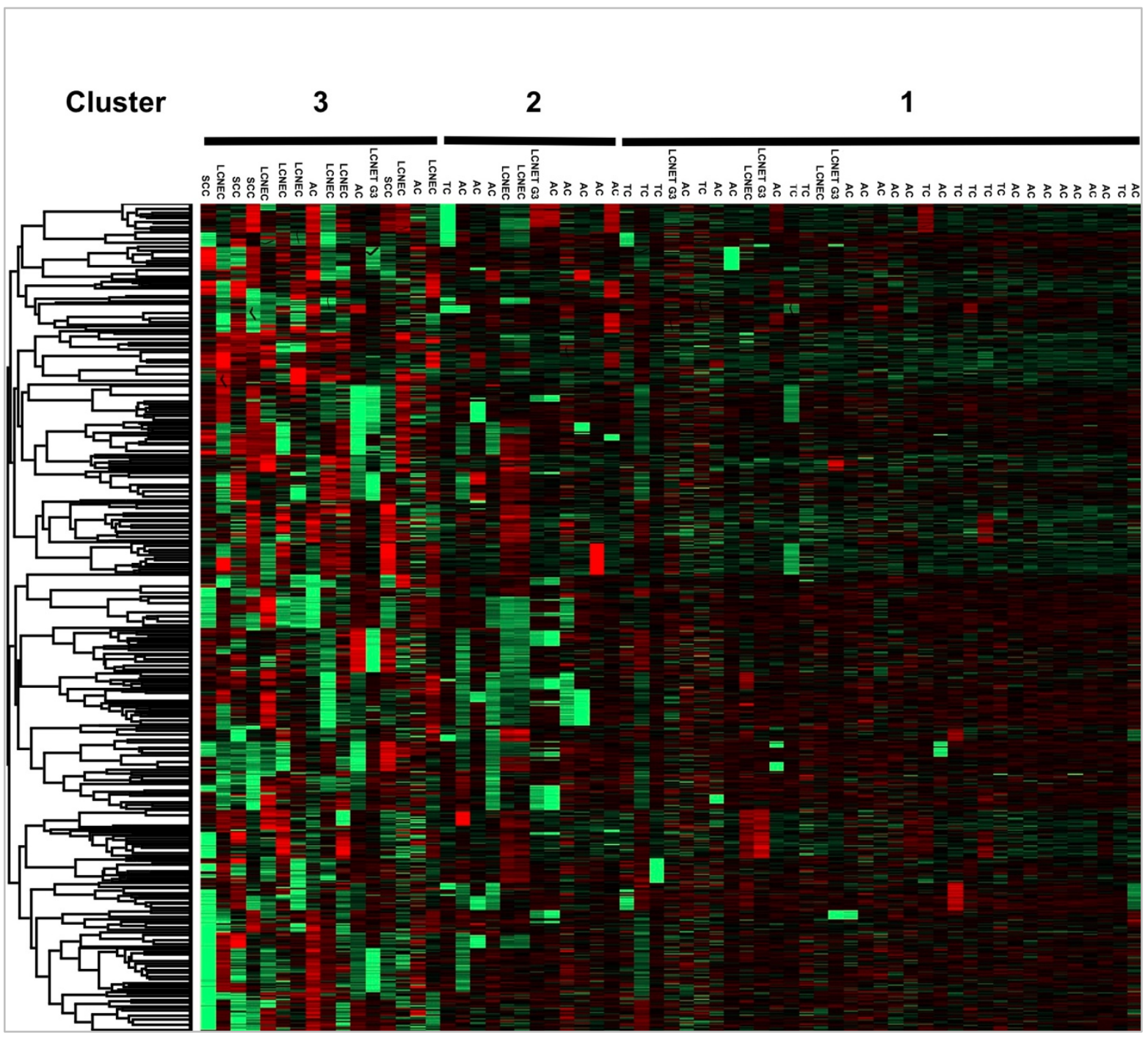

Figure 34: Unsupervised clustering of CNI data. Thymic NET fall into three major molecular groups. 8 LCNEC and 3 AC show cluster infidelity: These outlier AC fall into cluster 3 together with many highly genetically aberrant cases. The outlier LCNEC fall into cluster 2 and 1, which contain cases with moderate and low genomic instability.

\subsection{NET G3 versus NEC in Thymic NET}

\subsubsection{The Immunohistochemical Profile of NET G3}

Given the significant cluster inconsistency of LCNEC, we next analyzed the five LCNEC cases in the CNI low and $\mathrm{CNI}_{\text {int }}$ clusters and compared them to the six LCNEC cases in the $\mathrm{CNI}_{\text {high }}$ cluster. As mentioned in chapter 1, the GEP-NET grading system encompasses a fifth category called NET G3. The identification of well-differentiated NET G3 in the gastro-entero-pancreatic system can be facilitated through a specific immunohistochemical profile corresponding with specific mutations (Tang et al. 2016a). This immunohistochemical profile was stained on the above mentioned eleven 
LCNEC to discover if NET G3 can be uncovered in thymic NET with this method, and if the cluster-inconsistent LCNEC are examples of NET G3.

To reiterate here, NET G3 of the gastro-entero-pancreatic system express negative ATRX or DAXX (due to a mutually exclusive loss of these proteins), positive SSTR2A, positive RB1 and negative p53 immunolabeling (Jiao et al. 2011; Marinoni et al. 2014; Konukiewitz et al. 2017). NEC often display the opposite immunohistochemical profile: positive ATRX and DAXX, negative SSTR2A, negative RB1 and positive p53 immunolabeling. Negative RB1 immunolabeling is due to loss mutations within NEC tumor cells. Positive immunolabeling of p53 is caused by mutations that lead to p53 accumulation within the cells.

All stains were performed on microtome-cut tumor sections, mounted on microscope slides. ATRX and DAXX were stained by courtesy of the UMG molecular pathology lab. SSTR2A, RB1 and p53 were stained by the immunohistochemistry department of the UMG.

\subsubsection{NET G3 versus NEC Immunohistochemistry and Histologic Features}

Stain evaluations are depicted in chapter 4.7. An overview of the immunohistochemical results is listed in Table 3. SSTR2A did not demonstrate immunoreactivity in any thymic NET. Tumors $A$ and $H$ showed the expected immunolabeling associated with NEC and tumor D presented the expected immunolabeling associated with NET G3. The immunolabeling results of the remaining eight LCNEC did not match the expected profiles of NET G3 or NEC. Nonetheless, the tumors that we labeled NET G3: Tumors C, D, G, I, and J showed at least two of the following: low ki67 labeling index, low mitotic count, low CNI score, negative p53 immunoreactivity or a carcinoid morphology. On the other hand, the LCNEC that we labeled NEC, exhibited at least two of the following: elevated ki67 LI and mitotic count, high CNI scores, positive p53 immunoreactivity and carcinoma/high-grade morphology. The correlation of these parameters at low or high ends of the spectrum, suggests that there may be an additional subgroup among LCNEC.

\subsubsection{EZH2 and Chromogranin A in LCNEC}

As part of a bigger immunohistochemical analysis of the thymic neuroendocrine tumors, EZH2 and Chromogranin A immunohistochemistry expression was evaluated. The staining process and evaluation system are explained in chapter 4.7.1. In addition to the observations described in the paragraph above, expression of EZH2 and 
Chromogranin A showed a conspicuous pattern amongst large cell neuroendocrine carcinoma (Table 3).

Of the eleven LCNEC, all NET G3 (LCNEC C, D, G, I and J), showed negative EZH2 immunolabeling. On the other hand, 5 of 6 LCNEC (LCNEC B, E, F, H and K), showed positive EZH2 antibody reactivity. LCNEC A, a NEC, was an exception and also displayed negative EZH2 immunolabeling.

Furthermore, all NET G3 exhibited positive chromogranin A expression, while all NEC, with the exception of LCNEC B, exhibited negative chromogranin A expression. In conclusion, NET G3 and NEC showed protein expression at the opposite ends of the spectrum for EZH2 and chromogranin A: EZH2 was negative in all NETG G3 but positive in five of six LCNEC, while Chromogranin A was positive in all NET G3, but negative in five of six LCNEC.

\subsubsection{Next-Generation Sequencing of Five Genes}

With targeted panel sequencing, ATRX, NF1, KRAS and NRAS mutations were found in the eleven LCNEC (Table 3). An ATRX p.Q929E missense mutation was found in Tumor C, classified as NET G3. ATRX immunolabeling did not correspond with the presence of $A T R X$ mutations, as immunolabeling was positive in all cases, but only five out of six LCNEC were wildtype for ATRX. Tumor $C$ with the ATRX mutation also harbored three different RAS mutations: KRAS p.G12V, NRAS p.G13V, and NRAS p.Q61L. LCNEC A carried another NRAS p.Q61L mutation. All 11 LCNEC exhibited NF1 Y489C mutations despite the fact that the NF1 gene locus chr. 17q11.2 was unaltered in LCNEC, AC, and TC on low coverage WGS analysis. 
Table 3: Histological, immunohistochemical and molecular analysis of 11 LCNEC. 6 LCNEC are classified as NEC and 5 LCNEC are classified as NET G3 due to the markers listed in the table. All NEC portray at least two of the following: high ki67, high mitotic count, a carcinoma morphology, positive EZH2, positive $p 53$ and negative CgA immunolabeling and are genetically highly aberrant. All NET G3 portray at least two of the following: low ki67, low mitotic count, a carcinoid morphology, negative $\mathrm{EZH} 2$, negative $p 53$ and positive $\mathrm{CgA}$ immunolabeling and are genetically more benign.

\begin{tabular}{|c|c|c|c|c|c|c|c|c|c|c|c|c|c|c|c|}
\hline $\begin{array}{l}\text { WHO } \\
\text { Diagnosis }\end{array}$ & $\begin{array}{l}\text { ATRX } \\
\text { IHC }\end{array}$ & $\begin{array}{l}\text { ATRX Sequ. } \\
\text { Mutation; } \\
\text { Frequency (\%) }\end{array}$ & $\begin{array}{l}\text { DAXX } \\
\text { IHC }\end{array}$ & $\begin{array}{l}\text { SSTR2A } \\
\text { IHC }\end{array}$ & $\begin{array}{l}\text { RB1 } \\
\text { IHC }\end{array}$ & $\begin{array}{l}\text { P53 } \\
\text { IHC }\end{array}$ & $\begin{array}{l}\text { EZH2 } \\
\text { IHC }\end{array}$ & $\begin{array}{l}\text { CgA } \\
\text { IHC }\end{array}$ & $\begin{array}{l}\text { NF1 Sequ. } \\
\text { Mutation; } \\
\text { Frequency } \\
\text { (\%) }\end{array}$ & $\begin{array}{l}\text { Other Sequ. } \\
\text { Mutation; } \\
\text { Frequency (\%) }\end{array}$ & $\begin{array}{l}\text { Ki67 } \\
(\%)\end{array}$ & Mitoses & CNI & Morphology & $\begin{array}{l}\text { NET } \\
\text { G3 or } \\
\text { NEC }\end{array}$ \\
\hline LCNEC A & + & n.d. & + & - & - & + & - & - & $\begin{array}{l}1466 \mathrm{~A}>\mathrm{G} \\
87.38\end{array}$ & $\begin{array}{l}\text { NRAS; } 182 A>T ; \\
5.13\end{array}$ & 52 & $30 / 10$ & 83.4 & carcinoid & NEC \\
\hline LCNEC B & + & n.d. & + & - & $(+)$ & - & + & + & $\begin{array}{l}1466 \mathrm{~A}>\mathrm{G} ; \\
81.07\end{array}$ & n.d. & 59 & $67 / 10$ & 70.2 & $\begin{array}{l}\text { carcinoid/ } \\
\text { carcinoma }\end{array}$ & NEC \\
\hline LCNEC C & + & $\begin{array}{l}2785 C>G ; \\
18.75\end{array}$ & - & - & - & - & - & + & $\begin{array}{l}1466 A>G ; \\
32.65\end{array}$ & $\begin{array}{l}\text { KRAS; 35G>T; } \\
18.57 \\
\text { NRAS; 182A }>T ; \\
58.91 \\
\text { NRAS; 38G }>\mathrm{T} ; \\
74.53\end{array}$ & n.d. & $27 / 10$ & 6.12 & carcinoid & NET G3 \\
\hline LCNEC D & + & n.d. & - & - & $(+)$ & - & - & + & $\begin{array}{l}1466 A>G ; \\
81.43\end{array}$ & n.d. & 15 & $16 / 10$ & 3.1 & carcinoid & NET G3 \\
\hline LCNEC E & + & n.d. & + & - & $(+)$ & - & + & - & $\begin{array}{l}1466 \mathrm{~A}>\mathrm{G} ; \\
82.3\end{array}$ & n.d. & 90 & $100 / 10$ & 80.1 & carcinoid & NEC \\
\hline LCNEC F & $(+)$ & n.d. & + & - & n.d. & - & + & - & $\begin{array}{l}1466 \mathrm{~A}>\mathrm{G} ; \\
75.8\end{array}$ & n.d. & 70 & $12 / 10$ & 70.6 & carcinoid & NEC \\
\hline LCNEC G & + & n.d. & + & - & $(+)$ & - & - & + & $\begin{array}{l}1466 \mathrm{~A}>\mathrm{G} ; \\
71.2\end{array}$ & n.d. & 16 & $16 / 10$ & 18.8 & carcinoid & NET G3 \\
\hline LCNEC H & + & n.d. & + & - & - & + & + & - & $\begin{array}{l}1466 \mathrm{~A}>\mathrm{G} ; \\
73.54\end{array}$ & n.d. & 70 & $8 / 10$ & 64 & carcinoma & NEC \\
\hline LCNEC I & + & n.d. & $(+)$ & - & + & - & $(+)$ & + & $\begin{array}{l}1466 A>G ; \\
83.24\end{array}$ & n.d. & 66 & $14 / 10$ & 9.643 & carcinoid & NET G3 \\
\hline LCNEC J & + & n.d. & $(+)$ & $(+)$ & + & - & $(+)$ & + & $\begin{array}{l}1466 \mathrm{~A}>\mathrm{G} ; \\
87.16\end{array}$ & n.d. & 20 & $11 / 10$ & 27.66 & $\begin{array}{l}\text { carcinoid/ } \\
\text { carcinoma }\end{array}$ & NET G3 \\
\hline LCNEC K & + & n.d. & + & - & + & + & + & - & $\begin{array}{l}1466 \mathrm{~A}>\mathrm{G} ; \\
75.7\end{array}$ & n.d. & 55 & n.d. & n.d. & carcinoma & NEC \\
\hline
\end{tabular}

+, positive expression; (+) weak expression; -, negative expression; n.d., no data

\subsection{Calculating Cutoffs with Cox Proportional Hazard Regressions}

\subsubsection{Cox Hazard Regressions and Survival Analysis in Statistica}

Parameter cutoffs were calculated for mitotic count, ki67 and CNI using cox proportional hazard regressions. The goal was to find significant cutoffs of these parameters that place the TNET into significant prognostic groups, independent of WHO grade. The following survival analyses were performed with Kaplan-Meier diagrams and $p$-values were calculated with the log-rank test.

\subsubsection{Mitotic Count Cutoffs and Survival Analysis}

Within a cohort of 95 TNET (51 with available survival data), mitotic count cutoffs of $10,22,25,27,30,46$, and 100 showed statistically significant hazard ratios (Table 4). 10 mitoses are also a recognized cutoff in the WHO grading system, separating atypical carcinoids from high-grade NEC. The Pelosi NET classification defines 47 mitoses as a cutoff between NET G2 and NET G3. Our statistically significant finding of 46 mitoses indicated a similar cutoff. 
Table 4: Mitotic count cutoffs calculated with cox hazard regressions in 95 TNET. The mitoses cutoffs marked with a star, mark the statistically significant cutoff values, dividing TNET into statistically significant prognostic groups. A mitotic count of 10 and 30 show the strongest hazard ratios and p-values.

\begin{tabular}{|c|c|c|c|}
\hline \multicolumn{2}{|c|}{$\begin{array}{l}\text { Mitoses Cutoff } \\
\text { Group1 vs. Group2 }\end{array}$} & \multirow{2}{*}{$\begin{array}{l}\text { Hazard Ratio } \\
0.445671\end{array}$} & \multirow{2}{*}{$\begin{array}{l}\text { P-value } \\
0.197350\end{array}$} \\
\hline$<2$ & $\geq 2$ & & \\
\hline$<3$ & $\geq 3$ & 0.471906 & 0.141705 \\
\hline$<4$ & $\geq 4$ & 0.642585 & 0.321066 \\
\hline$<5$ & $\geq 5$ & 0.578801 & 0.209110 \\
\hline$<6$ & $\geq 6$ & 0.62689 & 0.277588 \\
\hline$<7$ & $\geq 7$ & 0.718048 & 0.4418 \\
\hline$<8$ & $\geq 8$ & 0.914107 & 0.837542 \\
\hline$<9$ & $\geq 9$ & 0.621140 & 0.279168 \\
\hline$<10$ & $\geq 10$ * & 0.382218 & 0.030749 \\
\hline$<12$ & $\geq 12$ & 0.453275 & 0.082582 \\
\hline$<19$ & $\geq 19$ & 0.407791 & 0.050775 \\
\hline$<20$ & $\geq 20$ & 0.480310 & 0.123988 \\
\hline$<22$ & $\geq 22$ * & 0.331552 & 0.023893 \\
\hline$<25$ & $\geq 25$ * & 0.335104 & 0.036246 \\
\hline$<27$ & $\geq 27^{*}$ & 0.112993 & 0.000082 \\
\hline$<30$ & $\geq 30$ * & 0.021240 & 0.000023 \\
\hline$<46$ & $\geq 46$ * & 0.039368 & 0.000129 \\
\hline$<100$ & $\geq 100$ * & 0.064655 & 0.001757 \\
\hline
\end{tabular}

Interestingly, a mitotic count of 30 showed the most significant $p$-value $(0.000023)$ and the lowest hazard ratio (0.021) in our cohort, demonstrating that two significant groups can be formed within the mitotic count spectrum of high-grade NEC.

The survival of patients with tumors with a mitotic count under 30 had a significantly better prognosis than tumors with a mitotic count over 30 . Dividing the tumors into three mitotic groups of $<10,10-29$ and $>30$ mitoses, also showed a strong prognostic differentiation between groups $(p=0.00006)$ (Fig. 35). Again, tumors with more than 30 mitoses had unfortunate prognosis, in contrast to tumors with less than 10 mitoses, portraying the longest survival. 


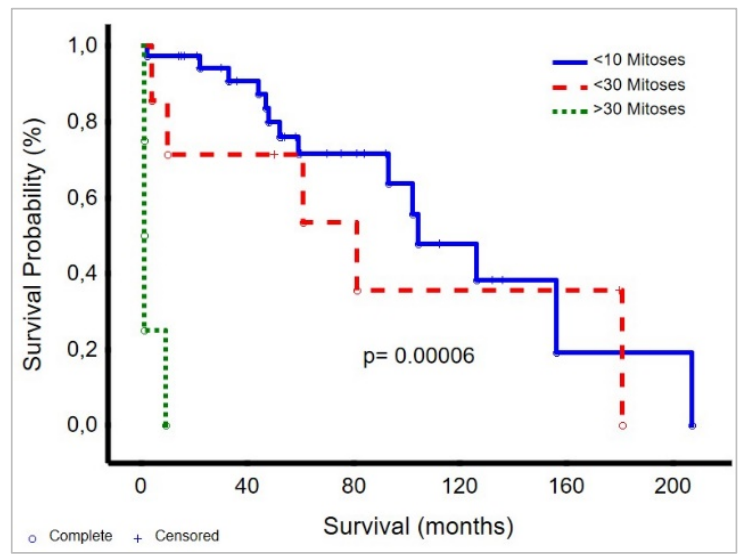

Figure 35: TNET with a mitotic count of $\geq 30$ associate with worse prognosis than TNET with a mitotic count of 10-29 or $<10$ mitoses. Creating three cutoffs, results in three significant prognostic groups.

\subsubsection{Ki67 Cutoffs and Survival Analysis}

61 TNET (18 with available survival data) were included in the hazard ratio analysis to find significant ki67 cutoffs. A ki67 labeling index of 7, 9 and 48 were statistically significant cutoff values (Table 5).

Table 5: Ki67 cutoffs calculated with cox hazard regressions in 61 TNET. The ki67 values marked with a star, mark the statistically significant cutoffs, dividing TNET into statistically significant prognostic groups. A ki67 of 9 shows the strongest hazard ratio and $p$-value.

\begin{tabular}{|ll|l|l|}
\hline \multicolumn{2}{|l|}{$\begin{array}{l}\text { Ki67 Cutoff } \\
\text { Group1 vs. Group2 }\end{array}$} & Hazard Ratio & P-value \\
\hline$<1$ & $\geq 1$ & 0.0 & 0.996715 \\
\hline$<2$ & $\geq 2$ & 0.356179 & 0.344478 \\
\hline$<3$ & $\geq 3$ & 0.263270 & 0.224161 \\
\hline$<4$ & $\geq 4$ & 0.191788 & 0.132710 \\
\hline$<6$ & $\geq 6$ & 0.142054 & 0.075534 \\
\hline$<7$ & $\geq 7 *$ & $\mathbf{0 . 1 0 2 5 9 9}$ & $\mathbf{0 . 0 3 8 3 4 1}$ \\
\hline$<9$ & $\geq 9 *$ & $\mathbf{0 . 0 6 9 7 8 2}$ & $\mathbf{0 . 0 1 5 8 2 6}$ \\
\hline$<48$ & $\geq 48 *$ & $\mathbf{0 . 1 5 7 5 0 6}$ & $\mathbf{0 . 0 3 3 5 9 6}$ \\
\hline$<60$ & $\geq 60$ & 0.273280 & 0.112281 \\
\hline$<70$ & $\geq 70$ & 0.867415 & 0.897622 \\
\hline
\end{tabular}

Ki67 cutoffs of 3 and 20, as defined in the ENETS classification, to separate NET G1 from NET G2 and NET G2 from NEC G3, did not show statistical significance. Similar to the findings of the mitoses' cutoffs, the ki67 labeling index also showed a high significant cutoff at 48 . This value lies in the middle/upper end of the ki67 spectrum, prevailing in the HGNEC range. Again, this suggests that a further subgroup may exist. A ki67 of $9 \%$ showed the strongest discernment of groups. In the Kaplan-Meier 
diagram, tumors with ki67 under 9\%, demonstrated longer survival than tumors with ki67 of $9 \%$ and above (Fig. 36).

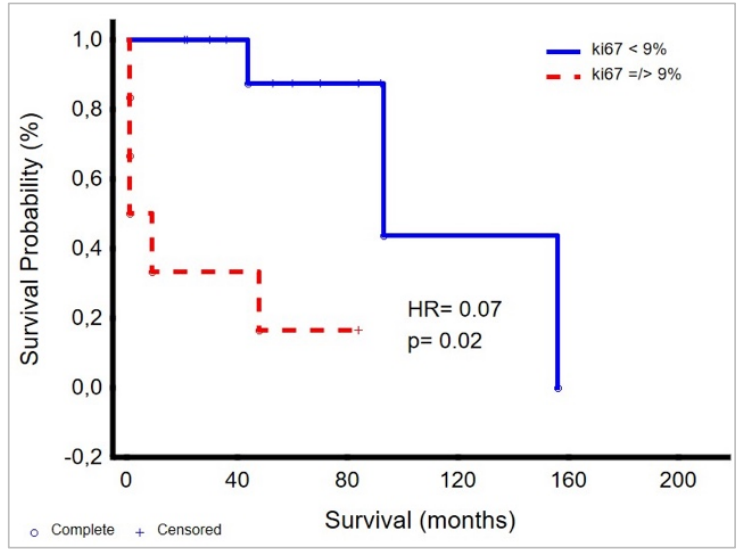

Figure 36: TNET with a ki67 of $\geq 9 \%$ associate with shorter survival probability than TNET with a ki67 of $<9 \%$. Separating the TNET into two groups, based on a ki67 </ $\geq 9 \%$ creates two significant prognostic groups.

\subsubsection{CNI Cutoffs and Survival Analysis}

CNI cutoff values were calculated with 63 TNET (18 with available survival data). The CNI score cutoffs 9, 11 , and 30 were statistically significant (Table 6). A CNI of 30 showed the lowest $p$-value $(0.018)$ and the lowest hazard ratio $(0.109)$. It is noteworthy, that again, the strongest CNI cutoff of 30 lies at the middle/upper end of the spectrum and encompasses a CNI value that would be expected of AC or LCNEC.

Table 6: CNI cutoffs calculated with cox hazard regressions in 63 TNET. The CNI values marked with a star, mark the statistically significant cutoffs. A CNI of 9 and 30 show the strongest hazard ratios and p-values.

\begin{tabular}{|ll|l|l|}
\hline \multicolumn{2}{|l|}{$\begin{array}{l}\text { CNI Cutoff } \\
\text { Group1 vs. Group2 }\end{array}$} & Hazard Ratio & P-value \\
\hline$<1$ & $\geq 1$ & 0.634413 & 0.67897 \\
\hline$<2$ & $\geq 2$ & 0.299655 & 0.267902 \\
\hline$<3$ & $\geq 3$ & 0.274625 & 0.240921 \\
\hline$<5$ & $\geq 5$ & 0.247132 & 0.202987 \\
\hline$<6$ & $\geq 6$ & 0.167749 & 0.105981 \\
\hline$<7$ & $\geq 7$ & 0.277873 & 0.154468 \\
\hline$<8$ & $\geq 8$ & 0.277873 & 0.154460 \\
\hline$<9$ & $\geq 9 *$ & $\mathbf{0 . 1 0 6 9 8 1}$ & $\mathbf{0 . 0 4 7 9 2 5}$ \\
\hline$<10$ & $\geq 10$ & 0.224915 & 0.106873 \\
\hline$<11$ & $\geq 11 *$ & $\mathbf{0 . 1 3 3 5 8 3}$ & $\mathbf{0 . 0 3 4 1 2 7}$ \\
\hline$<30$ & $\geq 30 *$ & $\mathbf{0 . 1 0 8 8 2 7}$ & $\mathbf{0 . 0 1 7 6 5 4}$ \\
\hline$<66$ & $\geq 66$ & 0.218882 & 0.099379 \\
\hline$<68$ & $\geq 68$ & 0.418420 & 0.437463 \\
\hline$<70$ & $\geq 70$ & 3720015 & 0.99 \\
\hline
\end{tabular}


The survival of patients with tumors with a CNI under 30 was longer than the survival of patients with tumors with a CNI of 30 and more (Fig. 37). Grouping the TNET into three groups of $\mathrm{CNI} \leq 9,10-30$ and $>30$, created three prognostically significant groups. Tumors with $\mathrm{CNI} \leq 9$ associated with the longest survival and tumors with $\mathrm{CNI}>30$ associated with the shortest survival.

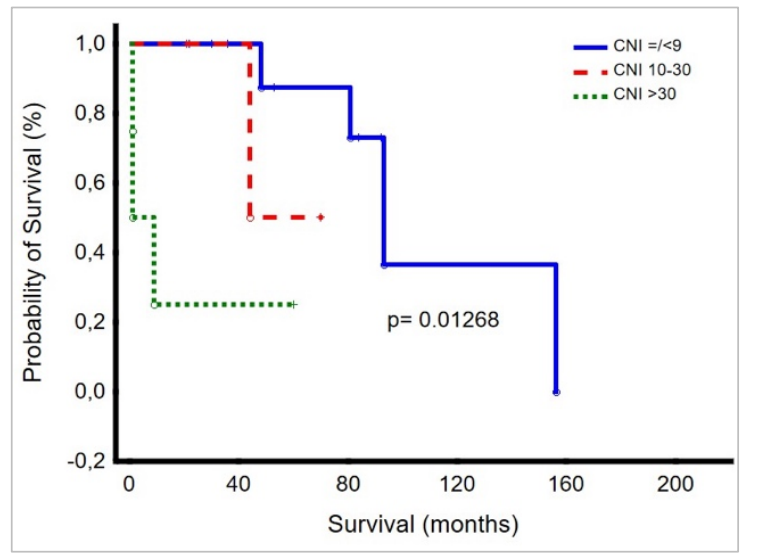

Figure 37: TNET with a CNI of $\geq 30$ associate with worse survival than TNET with a CNI of 10-<30 and $\leq 9$. Separating the TNET into three groups based on these CNI cutoff values, creates three significant prognostic groups.

All three parameters have in common that they feature statistically significant cutoffs in the middle to upper end of the spectrum. These cutoffs lie in a range that is encompassed by high-grade NEC (WHO mitoses cutoff for LCNEC and SCC: 20, our cutoff: 30; ENETS ki67 cutoff for NET G2 and NEC G3: 20, our cutoff: 48). Values that lie within the HGNEC range, yet divide this range into two prognostically significant groups, as our findings demonstrate, may indicate another subgroup amongst TNET. It is important to note that the LCNEC C, D, G, I and J, classified as NET G3 in Table 3 , all contained a mitotic count $<30$, a ki67 $<48$ (with one exception) and a $\mathrm{CNI}<30$.

\subsection{Immunohistochemical Stain Analysis}

\subsubsection{Tissue-Microarray and Evaluation of Stains}

Immunohistochemistry allows visualization of different biomarkers and exploration of protein/antigen expression or modification in tumor cells (Duraiyan et al. 2012). An immunohistochemical analysis of 21 stains was completed on the thymic neuroendocrine tumors to gain insight on grade-specific protein expression, and survival- or genetic-associated expression.

First, tissue microarray sections were constructed out of the FFPE tissue specimens by Targos Molecular Pathology $\mathrm{GmbH}$. The finished formalin-fixed and paraffinized tissue microarray (TMA) blocks were able to hold about 60 tumor samples, two tumor samples per case. The UMG molecular pathology lab cut the microarray sections with a microtome and mounted these on microscope slides for staining. The stains were 
conducted following protocol on the Dako Omnis advanced staining system or on the Dako Autostainer Link48.

Briefly, the TMA tumor specimens on the microscope slide were incubated in a primary antibody, which binds protein-specific antigens. A secondary antibody was added which binds the primary antibody. Because of an enzyme substrate reaction, a dye is released. This dye or stain is responsible for the positive immunolabeling and thus, signals the expression of the protein. A more detailed description of the staining protocol is described in chapter 3 . The number of stained and evaluated tumors varied in each case due to mechanical or systematic damage of the tissue cores during TMA production or during staining.

All stains were evaluated for staining intensity and the percentage of stained cells. Staining intensity ranged from no staining (0), to weak staining (1), to strong staining (2). The percentage of positive stained cells were grouped as $<25 \%$ of cells, $<50 \%$ of cells, and $>50 \%$ of cells. These parameters were used to group the stains into two groups: negative and positive immunolabeling (Table 7), or three groups: negative, medium, and positive immunolabeling (Table 8). A categorization of tumor stains into three groups was not always possible, due to a lack of cases within each expression group. A summary of all immunohistochemical stains and the evaluation of stains among the WHO subgroups is listed in Table 9.

Table 7: The two-tiered stain evaluation. Stains that were evaluated as $0,1<25$, or $2<25$ were considered to exhibit negative antibody expression. Stains that were evaluated as $1<50,1>50,2<50,2>50$ were considered to exhibit positive antibody expression.

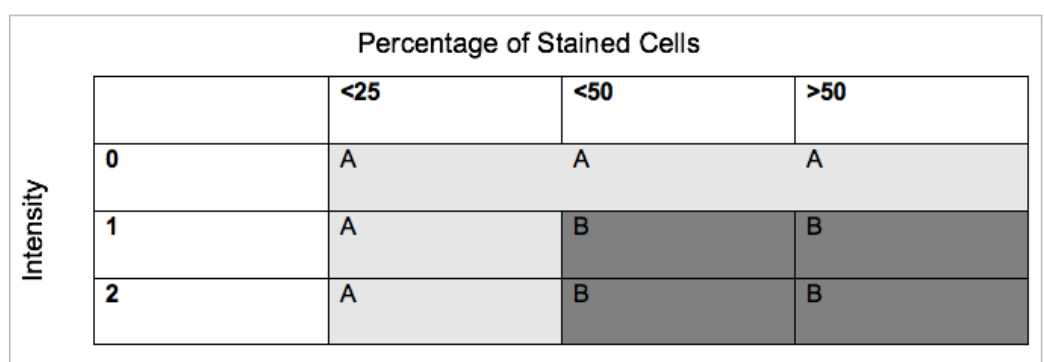

A: negative expression, B: positive expression 
Table 8: The three-tiered stain evaluation. Stains that were evaluated as 0 and $1<25$ were considered to exhibit negative antibody expression. Stains that were evaluated as $1<50$ and $2<25$ were considered to exhibit medium or weak antibody expression. Stains that were evaluated as $1>50,2<50$ and $2>50$ were considered to exhibit positive antibody expression.

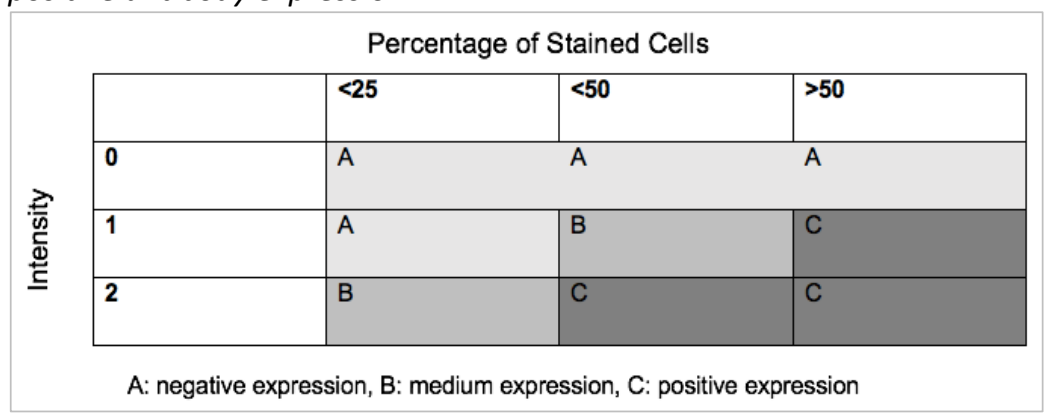

Table 9: Summary of all Immunohistochemical stains. The first row presents the 21 immunohistochemical stains. The left column presents the WHO subgroup. Each cell presents the percentage of negative and positive staining cases and the number of cases for each WHO subgroup for each stain.

\begin{tabular}{|c|c|c|c|c|c|c|c|c|c|c|c|c|c|}
\hline Histology & $\begin{array}{l}\text { Staining } \\
\text { intensity }\end{array}$ & ATRX & DAXX & SSTR2A & RB1 & P53 & c-Myc & SDHA & SDHB & \multicolumn{2}{|c|}{ EZH2 } & H3K27me3 & gH2AX \\
\hline \multirow[t]{2}{*}{ TC } & negative & $\begin{array}{l}67 \% \\
(6 / 9)\end{array}$ & $\begin{array}{l}44 \% \\
(4 / 9)\end{array}$ & $\begin{array}{l}100 \% \\
(10 / 10)\end{array}$ & $\begin{array}{l}67 \% \\
(6 / 9)\end{array}$ & $\begin{array}{l}80 \% \\
(8 / 10)\end{array}$ & $\begin{array}{l}75 \% \\
(6 / 8)\end{array}$ & $\begin{array}{l}56 \% \\
(5 / 9)\end{array}$ & $\begin{array}{l}60 \% \\
(6 / 10)\end{array}$ & $\begin{array}{l}100 \% \\
(9 / 9)\end{array}$ & & $\begin{array}{l}13 \% \\
(1 / 8)\end{array}$ & $\begin{array}{l}60 \% \\
(6 / 10) \\
\end{array}$ \\
\hline & positive & $\begin{array}{l}33 \% \\
(3 / 9)\end{array}$ & $\begin{array}{l}56 \% \\
(5 / 9)\end{array}$ & $\begin{array}{l}0 \% \\
(0 / 10)\end{array}$ & $\begin{array}{l}33 \% \\
(3 / 9)\end{array}$ & $\begin{array}{l}20 \% \\
(2 / 10)\end{array}$ & $\begin{array}{l}25 \% \\
(2 / 8)\end{array}$ & $\begin{array}{l}44 \% \\
(4 / 9)\end{array}$ & $\begin{array}{l}40 \% \\
(4 / 10)\end{array}$ & $\begin{array}{l}0 \% \\
(0 / 9)\end{array}$ & & $\begin{array}{l}87 \% \\
(7 / 8)\end{array}$ & $\begin{array}{l}40 \% \\
(4 / 10)\end{array}$ \\
\hline \multirow[t]{2}{*}{ AC } & negative & $\begin{array}{l}52 \% \\
(12 / 23)\end{array}$ & $\begin{array}{l}35 \% \\
(8 / 23)\end{array}$ & $\begin{array}{l}96 \% \\
(23 / 24)\end{array}$ & $\begin{array}{l}78 \% \\
(18 / 23)\end{array}$ & $\begin{array}{l}91 \% \\
(21 / 23)\end{array}$ & $\begin{array}{l}96 \% \\
(23 / 24)\end{array}$ & $\begin{array}{l}74 \% \\
(17 / 23)\end{array}$ & $\begin{array}{l}70 \% \\
(16 / 23)\end{array}$ & $\begin{array}{l}96 \% \\
(22 / 2\end{array}$ & 23) & $\begin{array}{l}61 \% \\
(14 / 23)\end{array}$ & $\begin{array}{l}74 \% \\
(17 / 23)\end{array}$ \\
\hline & positive & $\begin{array}{l}48 \% \\
(11 / 23)\end{array}$ & $\begin{array}{l}65 \% \\
(15 / 23)\end{array}$ & $\begin{array}{l}4 \% \\
(1 / 24)\end{array}$ & $\begin{array}{l}17 \% \\
(4 / 23)\end{array}$ & $\begin{array}{l}9 \% \\
(2 / 23)\end{array}$ & $\begin{array}{l}4 \% \\
(1 / 24)\end{array}$ & $\begin{array}{l}26 \% \\
(6 / 23)\end{array}$ & $\begin{array}{l}30 \% \\
(7 / 23)\end{array}$ & $\begin{array}{l}4 \% \\
(1 / 23\end{array}$ & $\begin{array}{l}35 \\
(9\end{array}$ & $\begin{array}{l}39 \% \\
(9 / 23)\end{array}$ & $\begin{array}{l}26 \% \\
(6 / 23)\end{array}$ \\
\hline \multirow[t]{2}{*}{ LCNEC } & negative & $\begin{array}{l}75 \% \\
(6 / 8)\end{array}$ & $\begin{array}{l}13 \% \\
(1 / 8)\end{array}$ & $\begin{array}{l}100 \% \\
(8 / 8)\end{array}$ & $\begin{array}{l}29 \% \\
(2 / 7)\end{array}$ & $\begin{array}{l}50 \% \\
(4 / 8)\end{array}$ & $\begin{array}{l}25 \% \\
(2 / 8)\end{array}$ & $\begin{array}{l}100 \% \\
(8 / 8)\end{array}$ & $\begin{array}{l}38 \% \\
(3 / 8)\end{array}$ & $\begin{array}{l}50 \% \\
(4 / 8)\end{array}$ & & $\begin{array}{l}50 \% \\
(4 / 8)\end{array}$ & $\begin{array}{l}38 \% \\
(3 / 8)\end{array}$ \\
\hline & positive & $\begin{array}{l}25 \% \\
(2 / 8)\end{array}$ & $\begin{array}{l}87 \% \\
(7 / 8)\end{array}$ & $\begin{array}{l}0 \% \\
(0 / 8)\end{array}$ & $\begin{array}{l}71 \% \\
(5 / 7)\end{array}$ & $\begin{array}{l}50 \% \\
(4 / 8)\end{array}$ & $\begin{array}{l}75 \% \\
(6 / 8)\end{array}$ & $\begin{array}{l}0 \% \\
(0 / 8)\end{array}$ & $\begin{array}{l}62 \% \\
(5 / 8)\end{array}$ & $\begin{array}{l}50 \% \\
(4 / 8)\end{array}$ & & $\begin{array}{l}50 \% \\
(4 / 8)\end{array}$ & $\begin{array}{l}62 \% \\
(5 / 8)\end{array}$ \\
\hline \multirow[t]{2}{*}{ SCC } & negative & $\begin{array}{l}100 \% \\
(3 / 3)\end{array}$ & $\begin{array}{l}33 \% \\
(1 / 3)\end{array}$ & $\begin{array}{l}100 \% \\
(3 / 3)\end{array}$ & $\begin{array}{l}100 \% \\
(3 / 3)\end{array}$ & $\begin{array}{l}33 \% \\
(1 / 3)\end{array}$ & $\begin{array}{l}100 \% \\
(3 / 3)\end{array}$ & $\begin{array}{l}100 \% \\
(3 / 3)\end{array}$ & $\begin{array}{l}67 \% \\
2 / 3\end{array}$ & $\begin{array}{l}0 \% \\
(0 / 3)\end{array}$ & & $\begin{array}{l}100 \% \\
(3 / 3)\end{array}$ & $\begin{array}{l}33 \% \\
(1 / 3)\end{array}$ \\
\hline & positive & $\begin{array}{l}0 \% \\
(0 / 3)\end{array}$ & $\begin{array}{l}67 \% \\
(2 / 3)\end{array}$ & $\begin{array}{l}0 \% \\
(0 / 3)\end{array}$ & $\begin{array}{l}0 \% \\
(0 / 3)\end{array}$ & $\begin{array}{l}67 \% \\
(2 / 3)\end{array}$ & $\begin{array}{l}0 \% \\
(0 / 3)\end{array}$ & $\begin{array}{l}0 \% \\
(0 / 3)\end{array}$ & $\begin{array}{l}33 \% \\
1 / 3\end{array}$ & $\begin{array}{l}100 \% \\
(3 / 3)\end{array}$ & & $\begin{array}{l}0 \% \\
(0 / 3)\end{array}$ & $\begin{array}{l}67 \% \\
(2 / 3)\end{array}$ \\
\hline Histology & $\begin{array}{l}\text { Staining } \\
\text { intensity }\end{array}$ & $\begin{array}{l}\text { Chromo } \\
\text { A }\end{array}$ & granin & Keratin & Calcitonin & Yap1 & TTF1 & Pax8 & Serot & & CDX2 & CD5 & CD117 \\
\hline \multirow[t]{2}{*}{ TC } & negative & $\begin{array}{l}0 \% \\
(0 / 10)\end{array}$ & & $\begin{array}{l}22 \% \\
(2 / 9)\end{array}$ & $\begin{array}{l}89 \% \\
(8 / 9)\end{array}$ & $\begin{array}{l}90 \% \\
(9 / 10)\end{array}$ & $\begin{array}{l}100 \% \\
(10 / 10)\end{array}$ & $\begin{array}{l}40 \% \\
(4 / 10)\end{array}$ & $\begin{array}{l}100 \% \\
(10 / 10\end{array}$ & & $\begin{array}{l}100 \% \\
(10 / 10)\end{array}$ & $\begin{array}{l}90 \% \\
(9 / 10)\end{array}$ & $\begin{array}{l}90 \% \\
(9 / 10)\end{array}$ \\
\hline & positive & $\begin{array}{l}100 \% \\
(10 / 10)\end{array}$ & & $\begin{array}{l}78 \% \\
(7 / 9)\end{array}$ & $\begin{array}{l}11 \% \\
(1 / 9)\end{array}$ & $\begin{array}{l}10 \% \\
(1 / 10)\end{array}$ & $\begin{array}{l}0 \% \\
(0 / 10)\end{array}$ & $\begin{array}{l}60 \% \\
(6 / 10)\end{array}$ & $\begin{array}{l}0 \% \\
(0 / 10)\end{array}$ & & $\begin{array}{l}0 \% \\
(0 / 10)\end{array}$ & $\begin{array}{l}10 \% \\
(1 / 10)\end{array}$ & $\begin{array}{l}10 \% \\
(1 / 10)\end{array}$ \\
\hline \multirow[t]{2}{*}{$A C$} & negative & $\begin{array}{l}0 \% \\
0 / 24\end{array}$ & & $\begin{array}{l}17 \% \\
(4 / 24)\end{array}$ & $\begin{array}{l}96 \% \\
(23 / 24)\end{array}$ & $\begin{array}{l}87 \% \\
(20 / 23)\end{array}$ & $\begin{array}{l}100 \% \\
(24 / 24)\end{array}$ & $\begin{array}{l}58 \% \\
(14 / 24)\end{array}$ & $\begin{array}{l}100 \% \\
(24 / 24\end{array}$ & & $\begin{array}{l}100 \% \\
(24 / 24)\end{array}$ & $\begin{array}{l}92 \% \\
(22 / 24)\end{array}$ & $\begin{array}{l}88 \% \\
(21 / 24)\end{array}$ \\
\hline & positive & $\begin{array}{l}100 \% \\
(24 / 24)\end{array}$ & & $\begin{array}{l}83 \% \\
(20 / 24)\end{array}$ & $\begin{array}{l}\% \% \\
(1 / 24)\end{array}$ & $\begin{array}{l}13 \% \\
(3 / 23)\end{array}$ & $\begin{array}{l}0 \% \\
(0 / 24)\end{array}$ & $\begin{array}{l}42 \% \\
(10 / 24)\end{array}$ & $\begin{array}{l}0 \% \\
(0 / 24)\end{array}$ & & $\begin{array}{l}0 \% \\
(0 / 24)\end{array}$ & $\begin{array}{l}8 \% \\
(2 / 24)\end{array}$ & $\begin{array}{l}13 \% \\
(3 / 24)\end{array}$ \\
\hline \multirow[t]{2}{*}{ LCNEC } & negative & $\begin{array}{l}50 \% \\
(4 / 8)\end{array}$ & & $\begin{array}{l}13 \% \\
(1 / 8)\end{array}$ & $\begin{array}{l}100 \% \\
(8 / 8)\end{array}$ & $\begin{array}{l}75 \% \\
(6 / 8)\end{array}$ & $\begin{array}{l}75 \% \\
(6 / 8)\end{array}$ & $\begin{array}{l}38 \% \\
(3 / 8)\end{array}$ & $\begin{array}{l}100 \% \\
(8 / 8)\end{array}$ & & $\begin{array}{l}88 \% \\
(7 / 8)\end{array}$ & $\begin{array}{l}63 \% \\
(5 / 8)\end{array}$ & $\begin{array}{l}75 \% \\
(6 / 8)\end{array}$ \\
\hline & positive & $\begin{array}{l}50 \% \\
(4 / 8)\end{array}$ & & $\begin{array}{l}88 \% \\
(7 / 8)\end{array}$ & $\begin{array}{l}0 \% \\
(0 / 8)\end{array}$ & $\begin{array}{l}25 \% \\
(2 / 8)\end{array}$ & $\begin{array}{l}25 \% \\
(2 / 8)\end{array}$ & $\begin{array}{l}63 \% \\
(5 / 8)\end{array}$ & $\begin{array}{l}0 \% \\
(0 / 8)\end{array}$ & & $\begin{array}{l}13 \% \\
(1 / 8)\end{array}$ & $\begin{array}{l}38 \% \\
(3 / 8)\end{array}$ & $\begin{array}{l}25 \% \\
(2 / 8)\end{array}$ \\
\hline \multirow[t]{2}{*}{ SCC } & negative & $\begin{array}{l}100 \% \\
(3 / 3)\end{array}$ & & $\begin{array}{l}33 \% \\
(1 / 3) \\
\end{array}$ & $\begin{array}{l}100 \% \\
(3 / 3)\end{array}$ & $\begin{array}{l}67 \% \\
(2 / 3) \\
\end{array}$ & $\begin{array}{l}100 \% \\
(3 / 3)\end{array}$ & $\begin{array}{l}100 \% \\
(3 / 3)\end{array}$ & $\begin{array}{l}100 \% \\
(3 / 3)\end{array}$ & & $\begin{array}{l}100 \% \\
(3 / 3)\end{array}$ & $\begin{array}{l}100 \% \\
(3 / 3)\end{array}$ & $\begin{array}{l}100 \% \\
(3 / 3)\end{array}$ \\
\hline & positive & $\begin{array}{l}0 \% \\
(0 / 3)\end{array}$ & & $\begin{array}{l}67 \% \\
(2 / 3)\end{array}$ & $\begin{array}{l}0 \% \\
(0 / 3)\end{array}$ & $\begin{array}{l}33 \% \\
(1 / 3)\end{array}$ & $\begin{array}{l}0 \% \\
(0 / 3)\end{array}$ & $\begin{array}{l}0 \% \\
(0 / 3)\end{array}$ & $\begin{array}{l}0 \% \\
(0 / 3)\end{array}$ & & $\begin{array}{l}0 \% \\
(0 / 3)\end{array}$ & $\begin{array}{l}0 \% \\
(0 / 3)\end{array}$ & $\begin{array}{l}0 \% \\
(0 / 3)\end{array}$ \\
\hline
\end{tabular}




\subsubsection{ATRX Immunohistochemical Analysis}

43 TNET (9 TC, 23 AC, 8 LCNEC, 3 SCC) were stained and evaluated for ATRX. The spectrum of staining intensity: negative/ 0 , weak/1 and strong/2 protein expression are shown in figure 38. The antibodies accumulated in the nucleus.

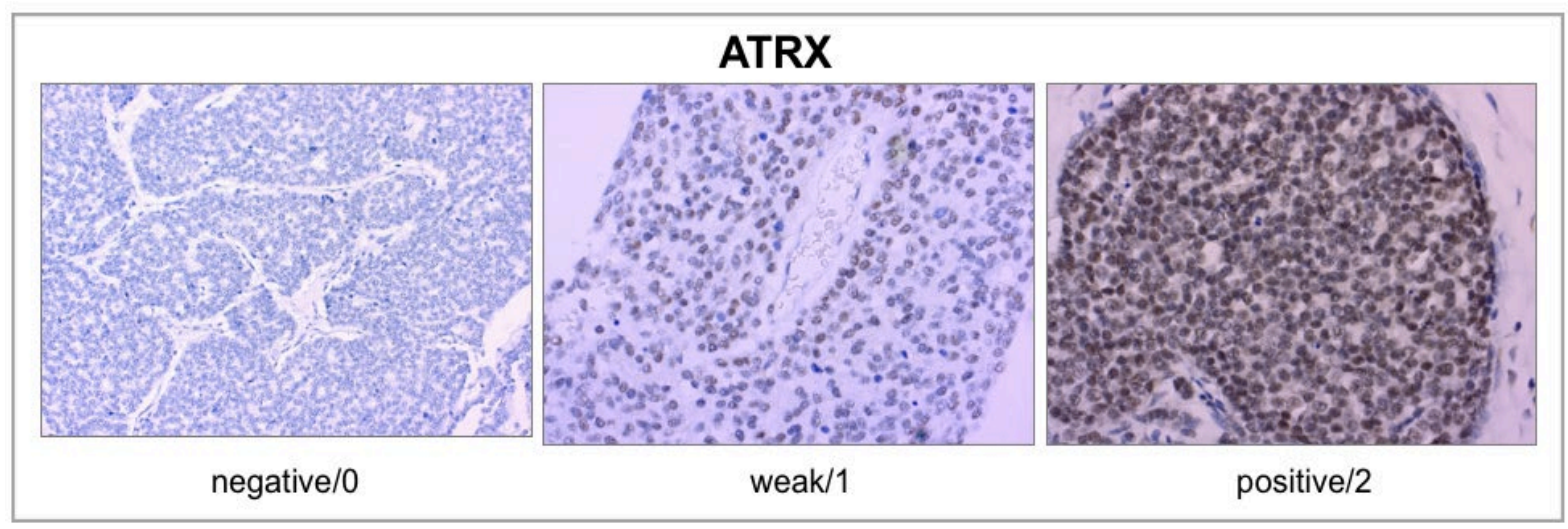

Figure 38: Immunohistochemistry staining intensity of ATRX. Left: ATRX negative tumor cells, AC, objective x20. Middle: ATRX weak tumor cells, AC, objective x40. Right: ATRX positive tumor cells, AC, objective x40.

In a two-tiered expression, 16 TNET (37\%) displayed positive protein expression, 27 (63\%) displayed no protein expression. Positive immunolabeling was present in 3 of 9 TC (33\%), 11 of 23 AC (48\%) and 2 of 8 of LCNEC (25\%). SCC showed negative immunolabeling in all three cases (Fig. 39). Applying the three-tiered evaluation system lead to 27 negative (6 TC, 12 AC, 6 LCNEC, 3 SCC), three medium (3 AC) and 13 positive ( 3 TC, 8 AC, 2 LCNEC) staining tumors.

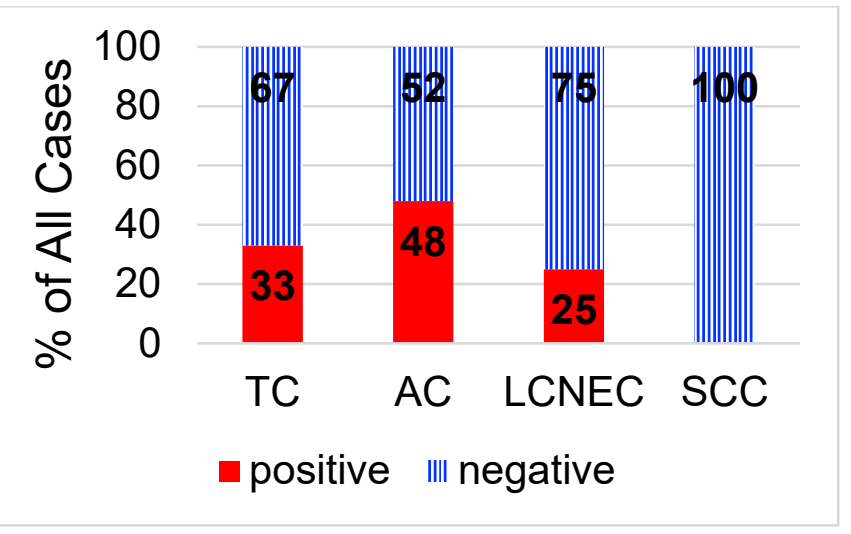

Figure 39: Distribution of ATRX expression among WHO subgroups. The columns represent the percentage of cases with positive or negative antibody expression. Y-axis: percentage of stained cells, X-axis: WHO subgroup.

Survival curves of the TNET grouped into positive and negative expression, did not show significant discernment in prognosis $(p=0.93$ ) (Fig. 40). The Kaplan-Meier diagram shows similar survival of both negative and positive groups until 90 months, then the "positive" survival curve drops to $0 \%$ survival probability. 
In the three-tiered survival curve in figure 41, staining intensity does not associate with survival and is not significant in the log-rank test $(p=0.53)$.

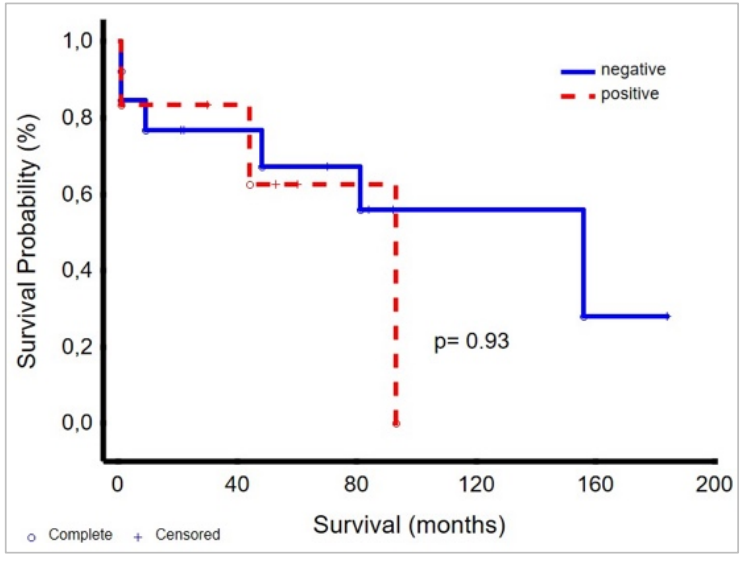

Figure 40: Survival analysis of TNET. The prognostic factor is two-tiered ATRX expression. Y-axis: proportion surviving. $X$-axis: time in months. ATRX expression does not correlate with survival

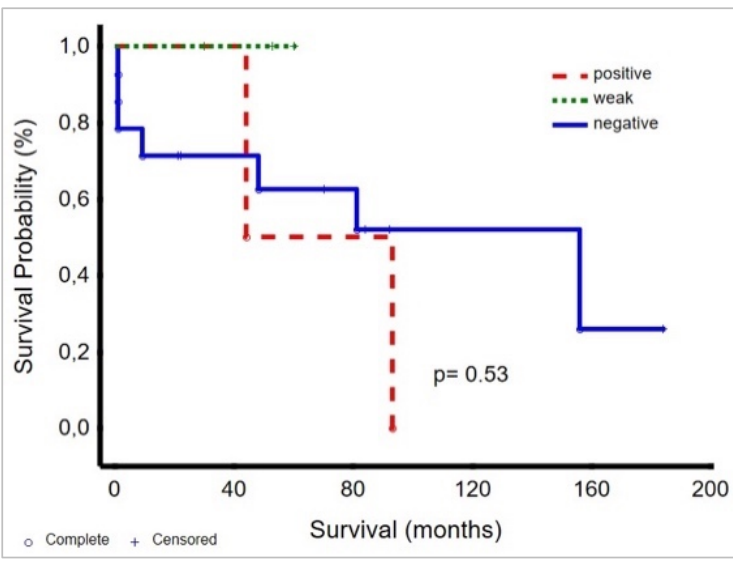

Figure 41: Survival analysis of TNET. The prognostic factor is the three-tiered ATRX expression. Y-axis: proportion surviving. $X$-axis: time in months.

Chromosomal aberrations and $\mathrm{CNI}$ did not correlate significantly with positive or negative ATRX expression (Fig. 42-43).

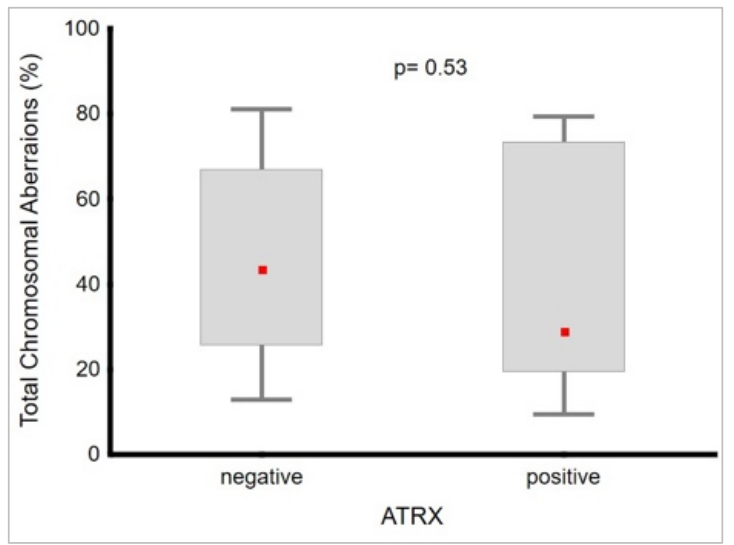

Figure 42: A correlation of ATRX expression with total chromosomal aberrations. Y-axis: percentage of chromosomal aberrations. X-axis: negative or positive ATRX staining.

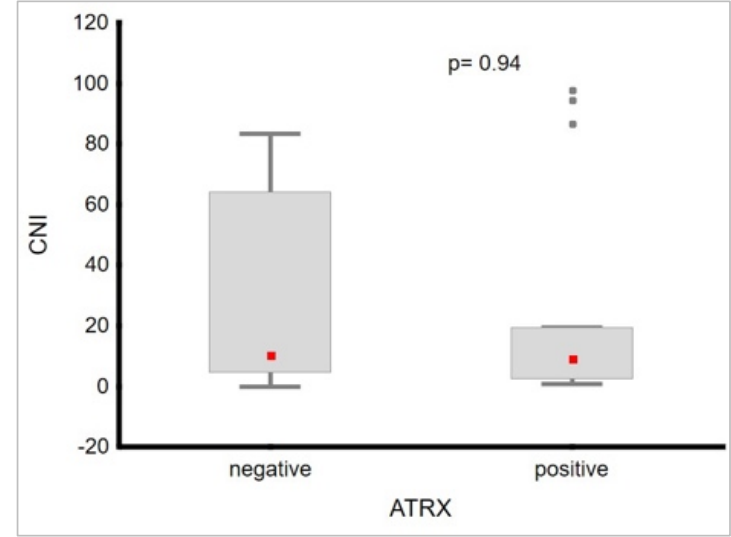

Figure 43: A correlation of ATRX expression with CNI. Y-axis: CNI score. $X$-axis: negative or positive ATRX staining. 


\subsubsection{DAXX Immunohistochemical Analysis}

DAXX antibody was stained on 43 TNET (9 TC, 23 AC, 8 LCNEC, 3 SCC). Figure 44 shows examples of negative/0, weak/1 and strong/2 immunolabeling of the tumor cells. Nuclear staining was observed in all tumor cells.

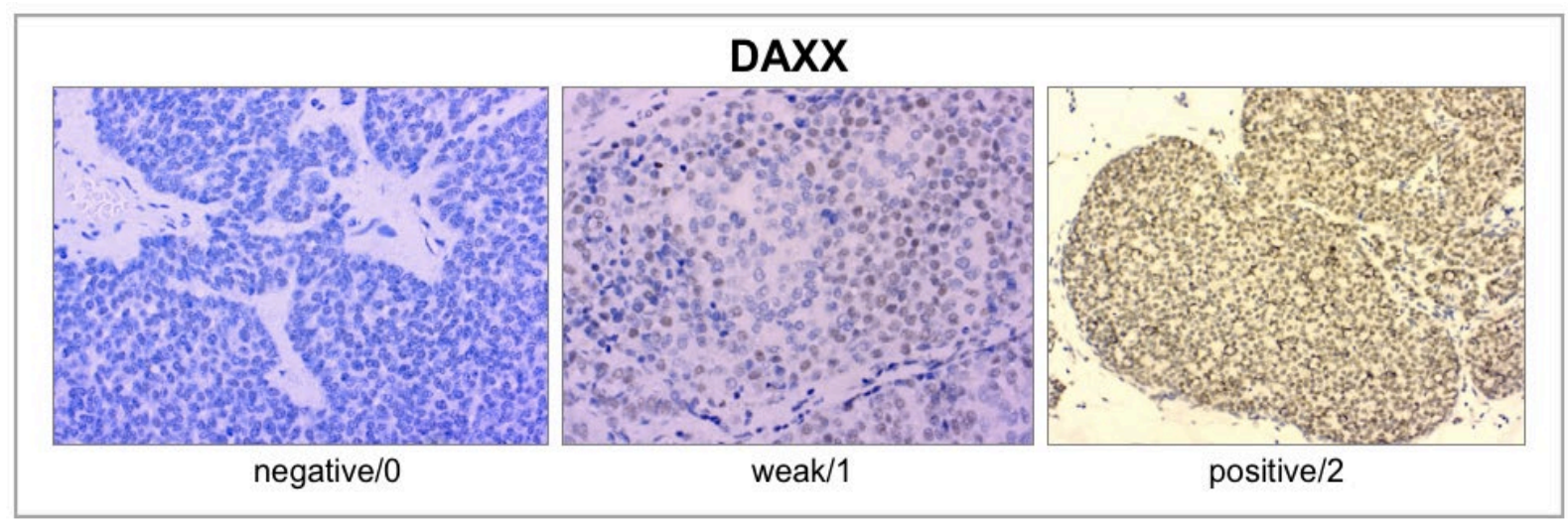

Figure 44: Immunohistochemistry staining intensity of DAXX. Left: DAXX negative tumor cells, AC, objective x40. Middle: DAXX weak tumor cells, LCNEC, objective X40. Right: DAXX positive tumor cells, TC, objective X20.

Altogether, 29 TNET (67\%) displayed positive immunoreactivity and 14 TNET (33\%) displayed negative immunoreactivity of DAXX. 5 of 9 TC (56\%), 15 of 23 AC (65\%), 7 of 8 LCNEC (87\%) and 2 of 3 SCC (67\%) showed positive immunolabeling (Fig. 45). In a three-tiered evaluation of IHC stains, 14 tumors (4 TC, 8 AC, 1 LCNEC, 1 SCC) displayed negative expression, 10 tumors (2 TC, 3 AC, 3 LCNEC, 2 SCC) displayed weak expression and 19 tumors (3 TC, 12 AC, 4 LCNEC) displayed positive expression.

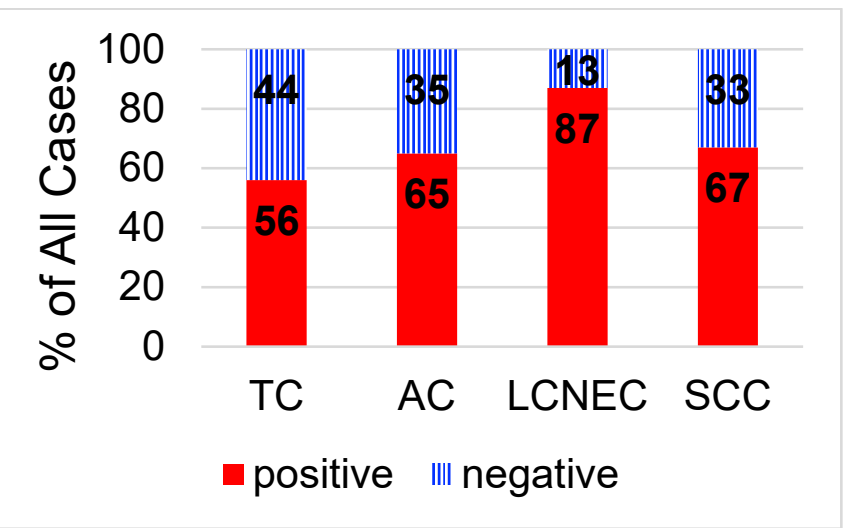

Figure 45: Distribution of DAXX expression among WHO subgroups. The columns represent the percentage of cases with positive or negative antibody expression. Y-axis: percentage of stained cells, X-axis: WHO subgroup.

The Kaplan-Meier curve in figure 46 presents tumors with positive staining to correspond with shorter survival than tumors with negative staining. This correlation was however, not statistically significant $(p=0.3)$. In figure 47 , DAXX expression is three-tiered, and shows tumors with weak staining to have the shortest survival, 
followed by tumors with positive staining. Tumors with negative expression, correlated with the longest overall survival $(p=0.07)$.

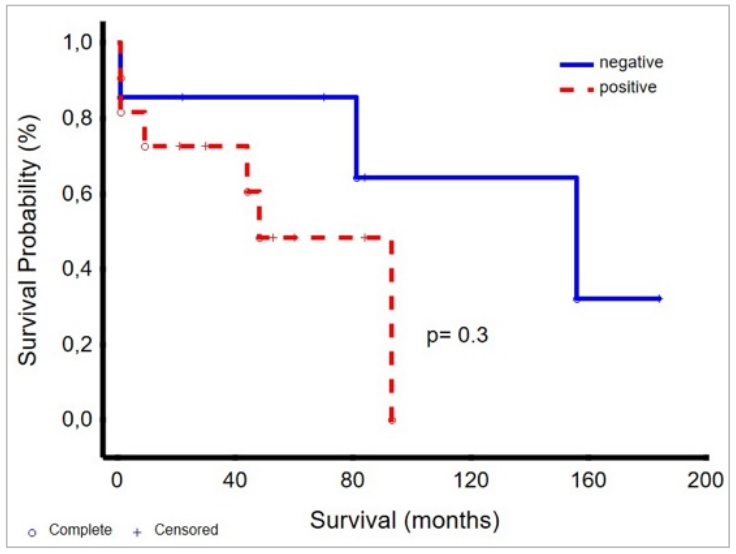

Figure 46: Survival analysis of TNET. The prognostic factor is two-tiered DAXX expression. $Y$ axis: proportion surviving. $X$-axis: time in months.

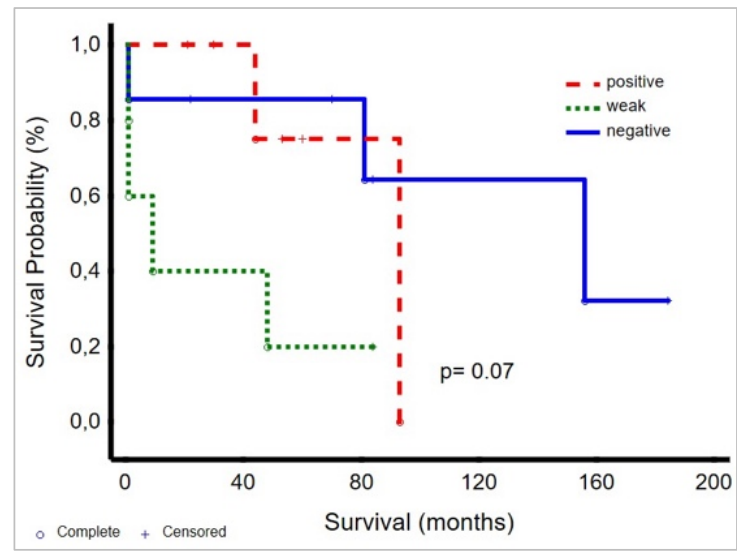

Figure 47: Survival analysis of TNET. The prognostic factor is three-tiered DAXX expression. Y-axis: proportion surviving. $X$-axis: time in months.

The number of chromosomal aberrations and the CNI score did not correlate significantly with DAXX expression (Fig. 48-49).

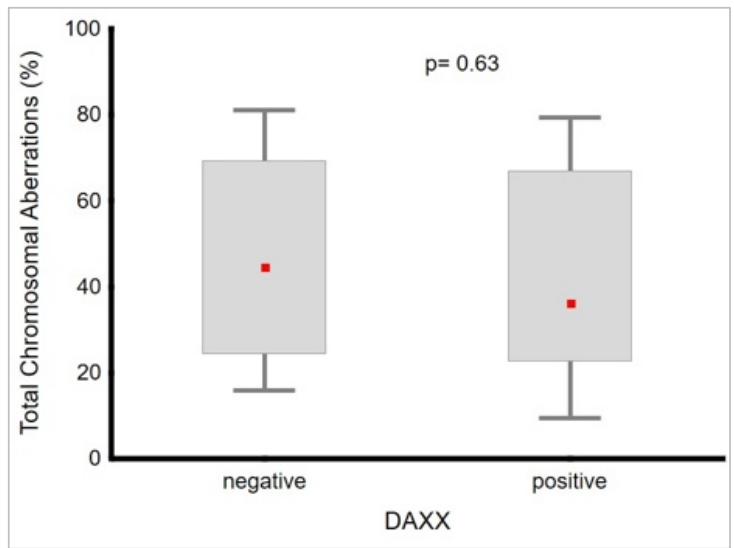

Figure 48: A correlation of DAXX expression with total chromosomal aberrations. Y-axis: percentage of chromosomal aberrations. $X$-axis: negative or positive DAXX staining.

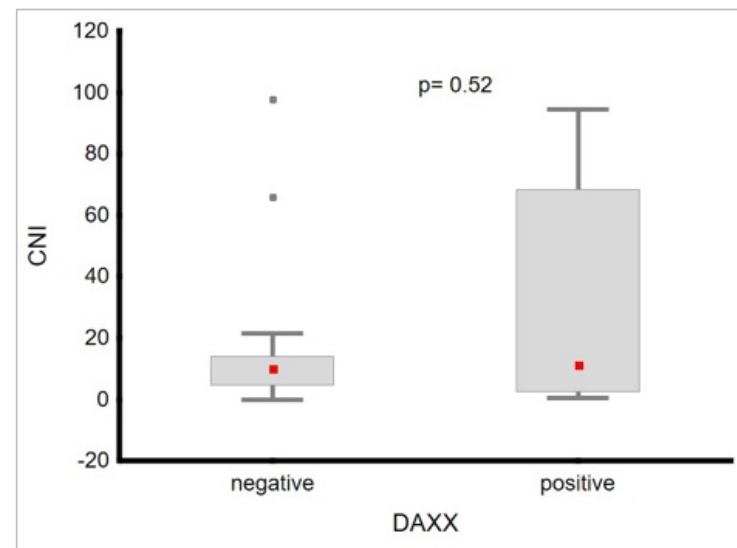

Figure 49: A correlation of DAXX expression with CNI. Y-axis: CNI score. X-axis: negative or positive $D A X X$ staining. 


\subsubsection{SSTR2A Immunohistochemical Analysis}

SSTR2A staining was performed on 45 TNET (10 TC, 24 AC, 8 LCNEC, 3 SCC). Cytoplasmic staining was observed. Examples of negative and positive expression are demonstrated in fig. 50. All TNET lacked SSTR2A expression, except for one atypical carcinoid (Fig. 51). SSTR2A expression was not a typical biomarker in our cohort as it is in gastro-intestinal and pancreatic NET. Figure 52 shows the survival curve of SSTR2A negative tumors. A comparison of two groups was not possible due to the lack of positive tumors.

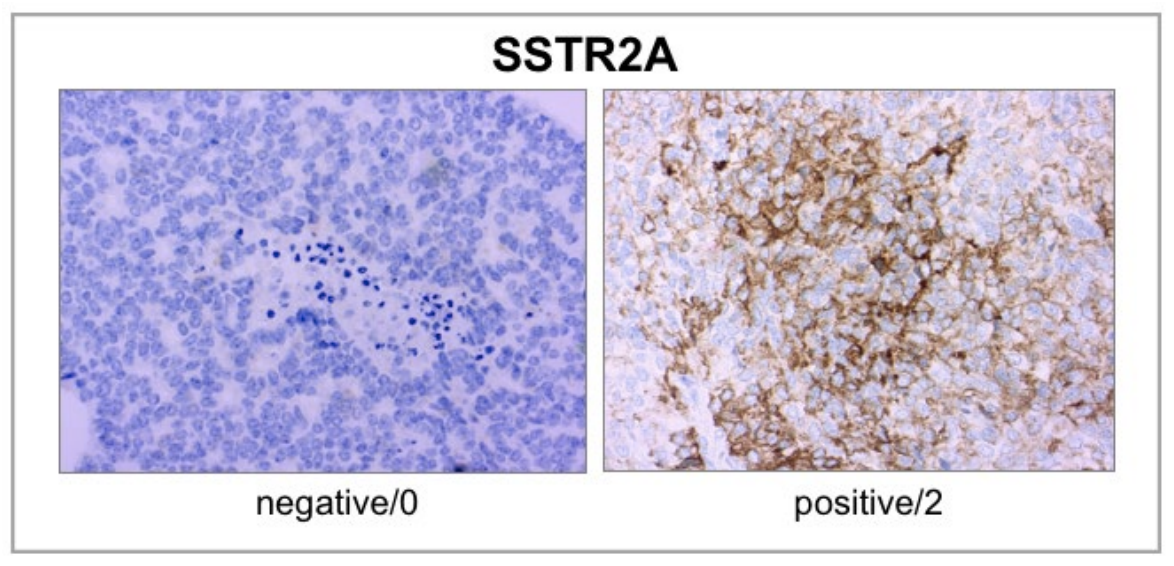

Figure 50: Immunohistochemistry staining intensity of SSTR2A. Left: SSTR2A negative tumor cells, AC, objective x40. Right: SSTR2A positive tumor cells, AC, objective $x 40$.

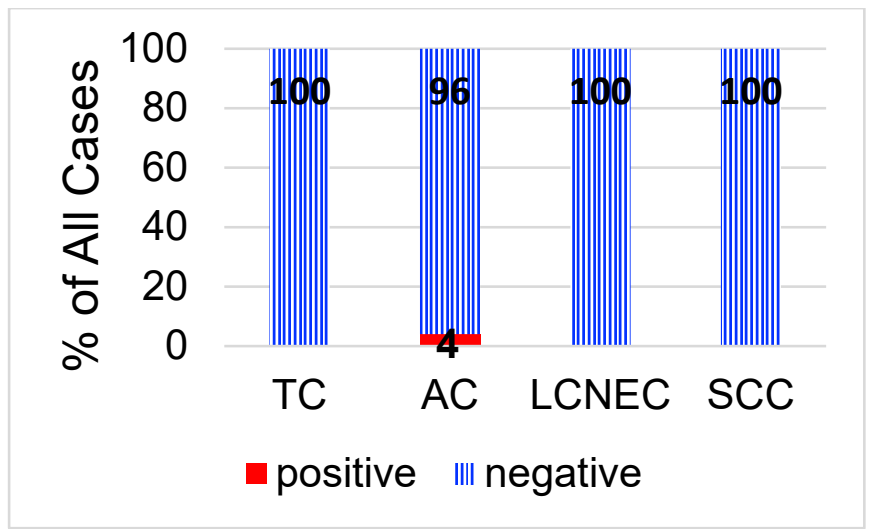

Figure 51: Distribution of SSTR2A expression among WHO subgroups. The columns represent the percentage of cases with positive or negative antibody expression. Y-axis: percentage of stained cells, X-axis: WHO subgroup. 


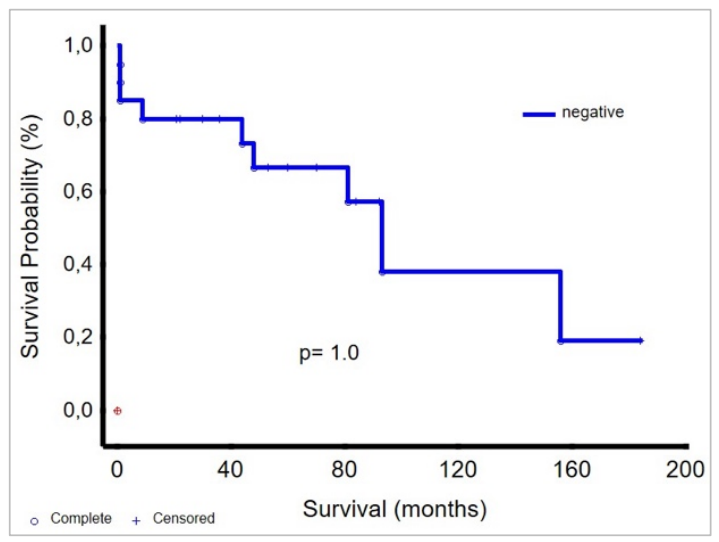

Figure 52: Survival analysis of TNET. The prognostic factor is two-tiered SSTR2A expression. Y-axis: proportion surviving. $X$-axis: time in months.

\subsubsection{RB1 Immunohistochemical Analysis}

RB1 immunostaining was performed on 42 TNET (9 TC, 23 AC, 7 LCNEC, 3 SCC). The stain, if present, was nuclear and/or cytoplasmic. Figure 53 shows examples of the color intensity spectrum of the antibody.

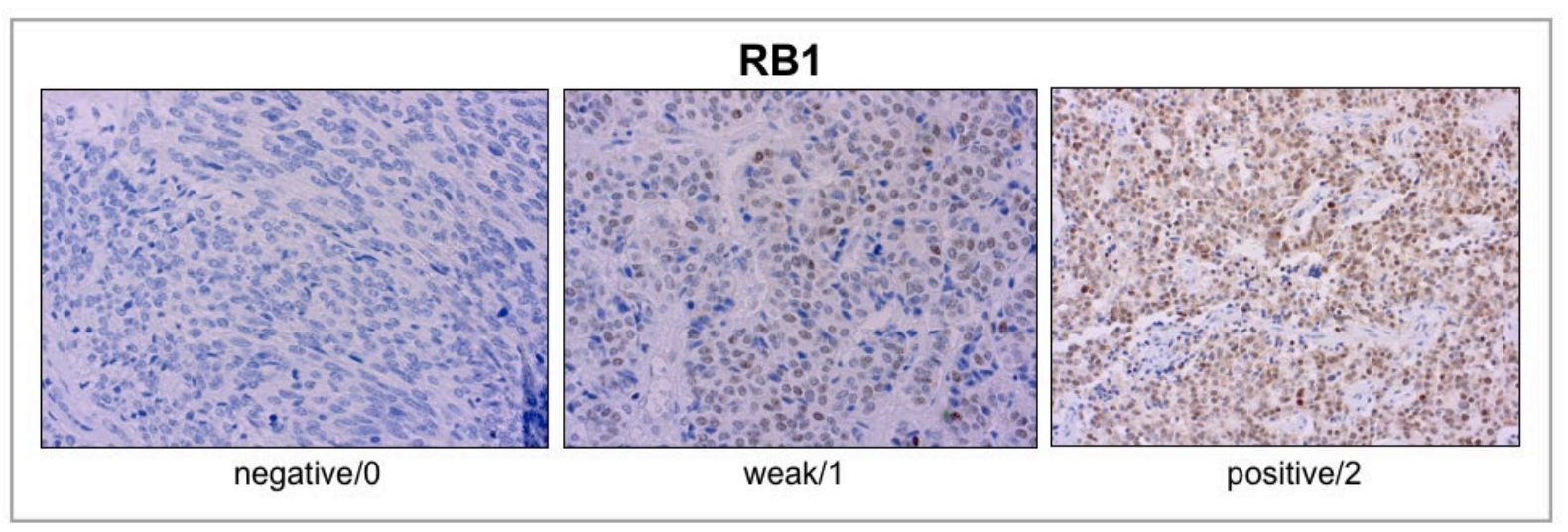

Figure 53: Immunohistochemistry staining intensity of RB1. Left: RB1 negative tumor cells, AC, objective x40. Middle: RB1 weak tumor cells, AC, objective x40. Right: RB1 positive tumor cells, TC, objective x20.

In all, 12 cases (29\%) displayed positive expression and 30 cases $(71 \%)$ displayed negative expression. 3 of 9 TC (33\%), 4 of 23 AC (17\%) and 5 of 7 LCNEC $(71 \%)$ expressed positive immunolabeling (Fig. 54). All SCC displayed negative RB1 protein expression. Categorizing RB1 expression in three levels, lead to 29 negative, four weak and eight positive tumors. 


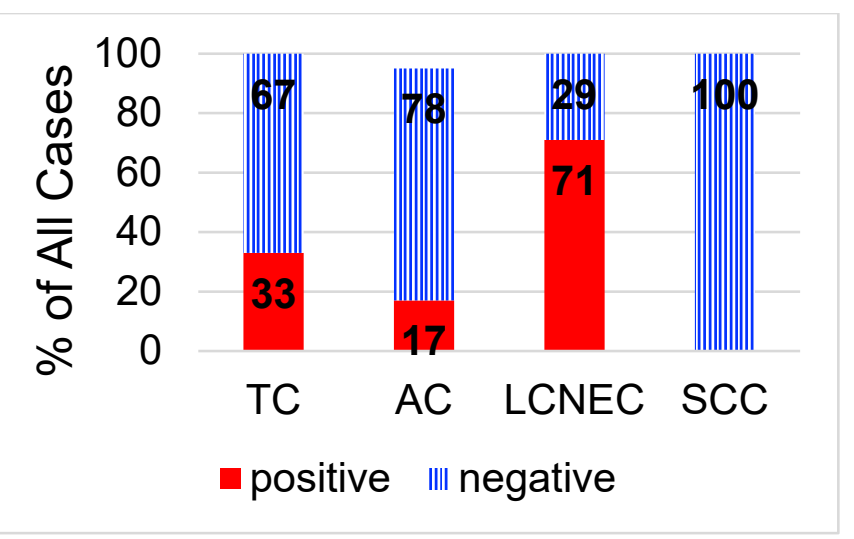

Figure 54: Distribution of RB1 expression among WHO subgroups. The columns represent the percentage of cases with positive or negative antibody expression. Y-axis: percentage of stained cells, X-axis: WHO subgroup.

The survival curve in figure 55 indicates that negative RB1 expression correlates with longer survival, however it is difficult to evaluate the "positive" curve because it ends at 60 months. The Kaplan-Meier analysis was not significant $(p=0.74)$. In figure 56 , the three-tiered survival analysis shows longest survival amongst tumors with weak RB1 staining, followed by negative RB1 staining. Tumors with positive staining associated with dismal prognosis.

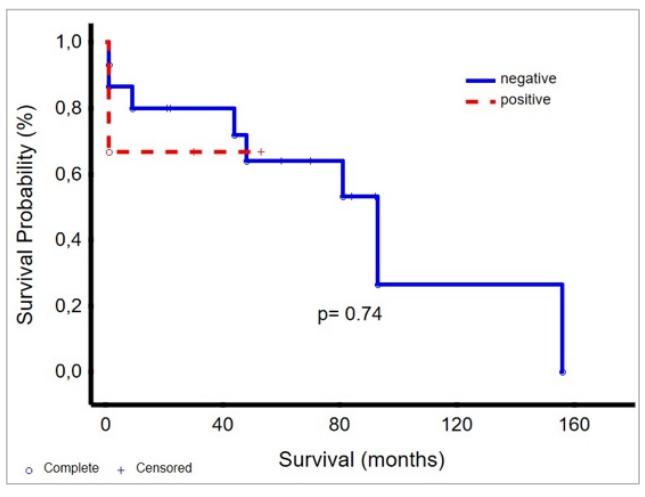

Figure 55: Survival analysis of TNET. The prognostic factor is two-tiered RB1 expression. Y-axis: proportion surviving. $X$ axis: time in months.

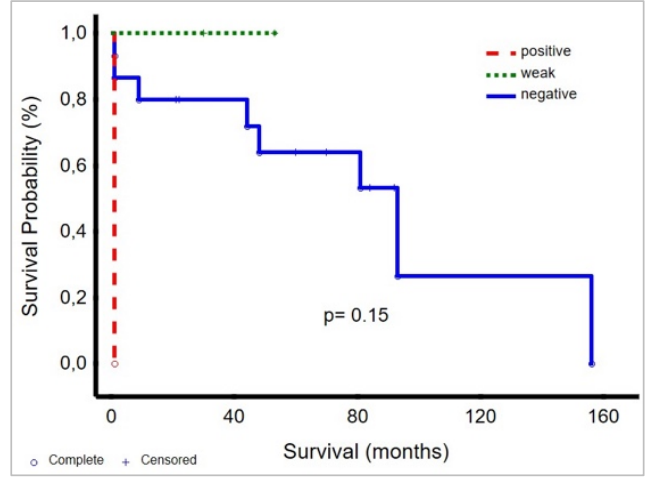

Figure 56: Survival analysis of TNET. The prognostic factor is three-tiered RB1 expression. $Y$-axis: proportion surviving. $X$ axis: time in months.

The boxplots in figures 57-58 do not show statistically significant correlations of RB1 staining and chromosomal aneuploidy, however, there was a trend to more aberrations with positive RB1 staining. 


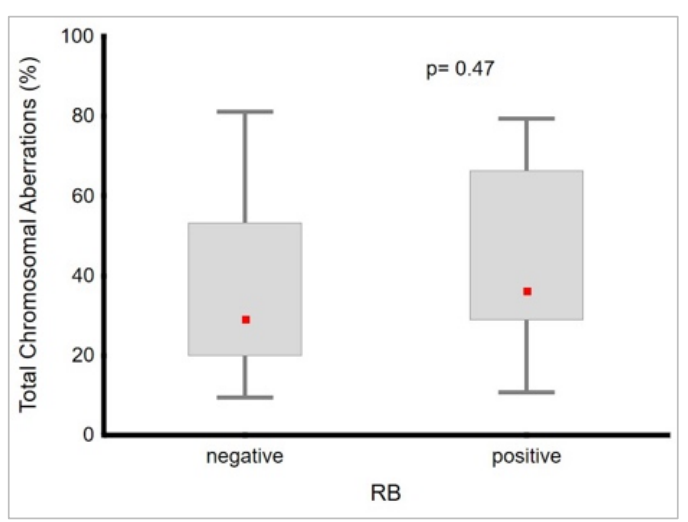

Figure 57: A correlation of RB1 expression with total chromosomal aberrations. Y-axis: percentage of chromosomal aberrations. $X$ axis: negative or positive RB1 staining.

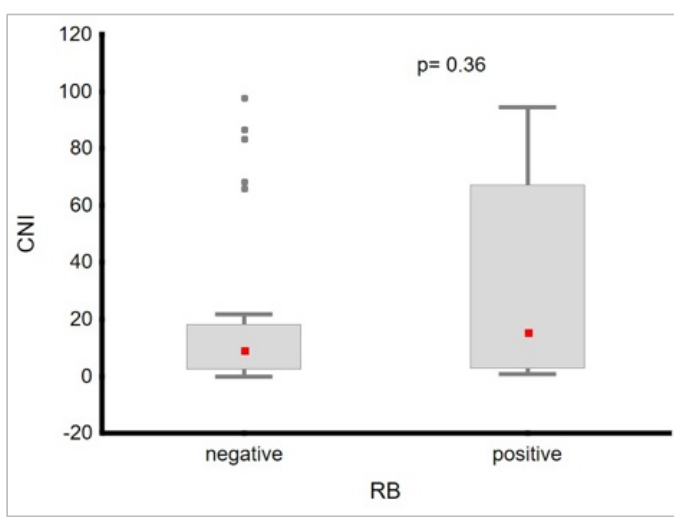

Figure 58: A correlation of RB1 expression with CNI score. Y-axis: CNI score. X-axis: negative or positive RB1 staining.

\subsubsection{P53 Immunohistochemical Analysis}

44 TNET (10 TC, 23 AC, 8 LCNEC, 3 SCC) were evaluated for p53 expression. P53 staining was found in the nucleus. Examples of staining intensity are depicted in figure 59.

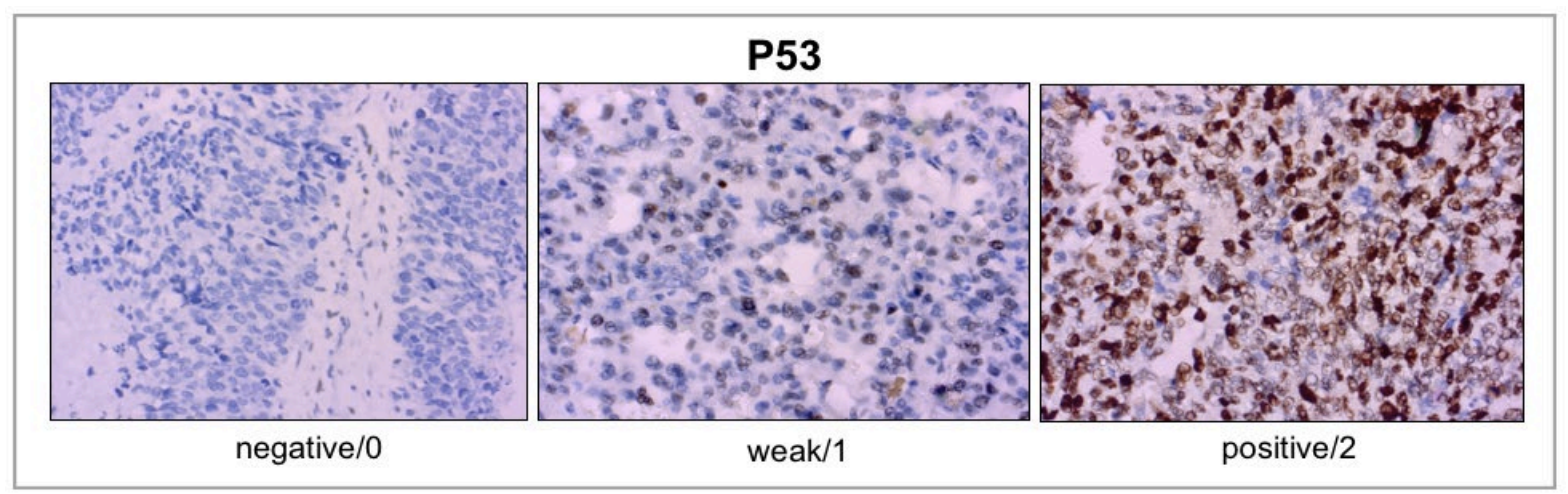

Figure 59: Immunohistochemistry staining intensity of P53. Left: P53 negative tumor cells, AC, objectivex40. Middle: P53 weak tumor cells, TC, objective x40. Right: P53 positive tumor cells, LCNEC, objective x40.

Altogether, ten cases (23\%) were positive for p53 antibody reactivity and 34 cases (77\%) were negative for p53 antibody reactivity. 2 of 10 TC (20\%), 2 of 23 AC (9\%), 4 of 8 LCNEC (50\%) and 2 of 3 SCC $(67 \%)$ displayed positive p53 protein expression (Fig. 60). Using a three-tiered evaluation, 29 TNET (8 TC, 16 AC, 4 LCNEC, 1 SCC) showed negative, nine showed weak (2, TC, 6 AC, 1 LCNEC) and six showed positive (1 AC, 3 LCNEC, 2 SCC) p53 protein expression. 


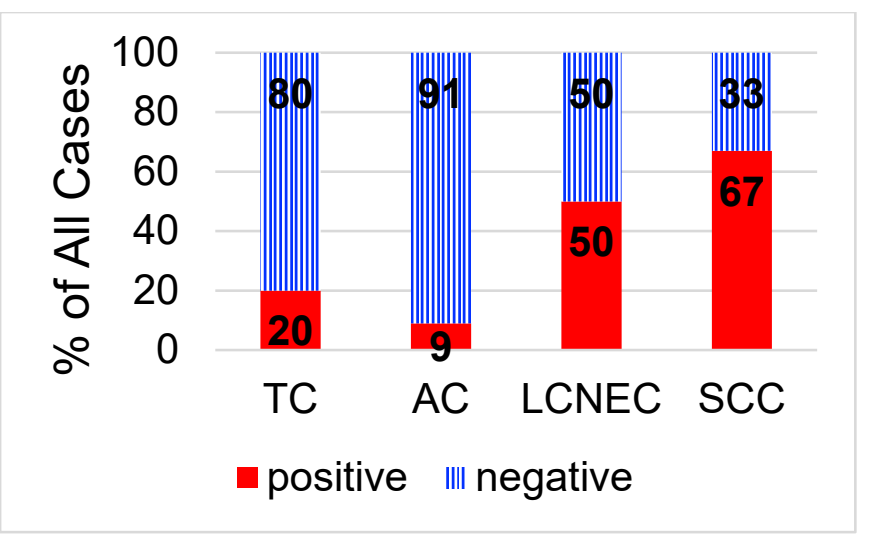

Figure 60: Distribution of P53 expression among WHO subgroups. The columns represent the percentage of cases with positive or negative antibody expression. Y-axis: percentage of stained cells, X-axis: WHO subgroup.

In the two-tiered Kaplan-Meier diagram, patients with p53 negative tumors had the longest overall survival. The curve shows a gradual decline over a time span of 200 months. Patients with p53 positive tumors had a very short overall survival, lasting no longer than 10 months (Fig. 61). The p-value between the two survival curves was 0.02 in the log-rank test. The survival curves in figure 62 did not show statistically significant results. However, the same trend as the two-tiered survival curve can be observed. Patients with negative staining tumors had the longest overall survival. Patients of positive staining tumors again, had the shortest survival time, lasting no longer than 80 months.

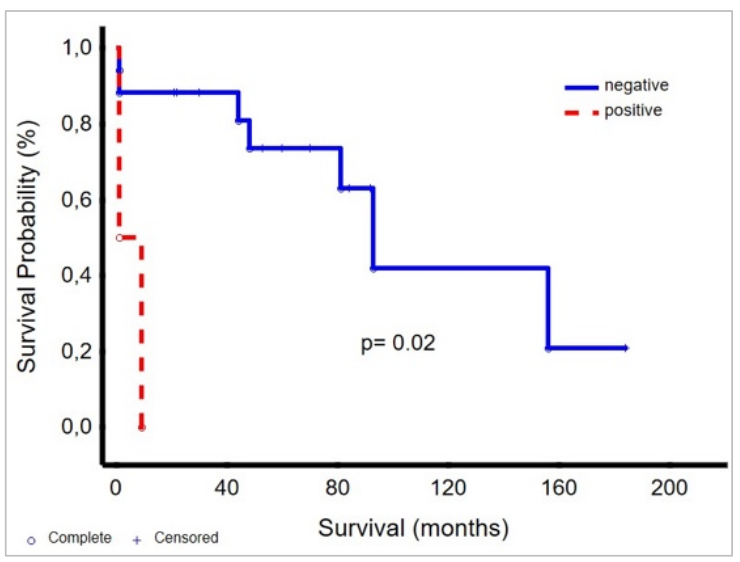

Figure 61: Survival analysis of TNET. The prognostic factor is two-tiered P53 expression. $Y$ axis: proportion surviving. $X$-axis: time in months.

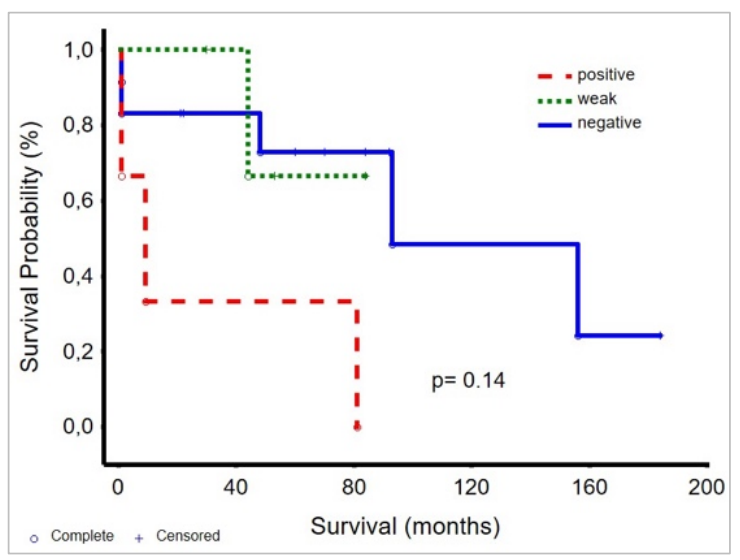

Figure 62: Survival Analysis of TNET. The prognostic factor is the three-tiered $P 53$ expression. $Y$-axis: proportion surviving. $X$-axis: time in months.

The boxplots in figures 63-64 indicate that p53 positive tumors had a wide range, and often more chromosomal aberrations and CNI scores than p53 negative tumors. In this cohort, p53 did not correlate significantly with genetic alterations. 


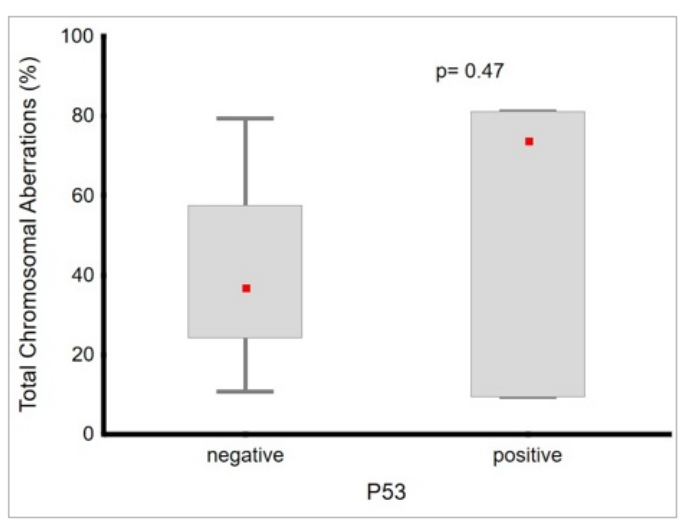

Figure 63: A correlation of P53 expression with total chromosomal aberrations. Y-axis: percentage of chromosomal aberrations. $X$ axis: negative or positive P53 staining.

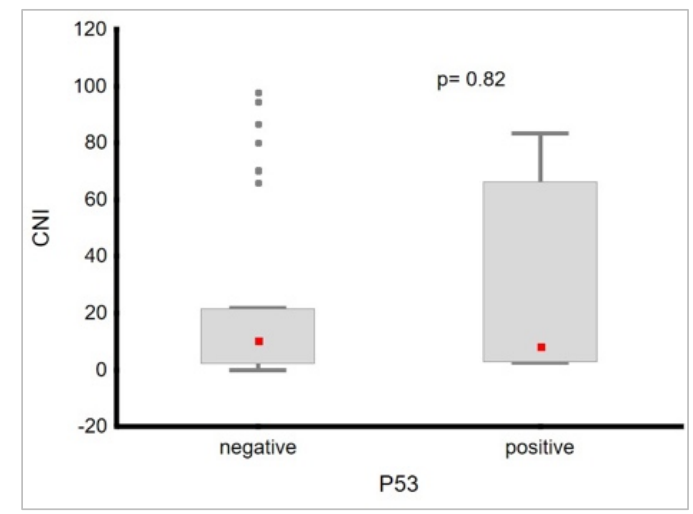

Figure 64: A correlation of P53 expression with CNI score. Y-axis: CNI score. X-axis: negative or positive $P 53$ staining.

\subsubsection{C-Myc Immunohistochemical Analysis}

C-Myc was stained on 43 TNET (8 TC, 24 AC, 8 LCNEC, 3 SCC). The staining intensity spectrum is demonstrated in figure 65 . C-Myc antibodies accumulated in the nucleus.

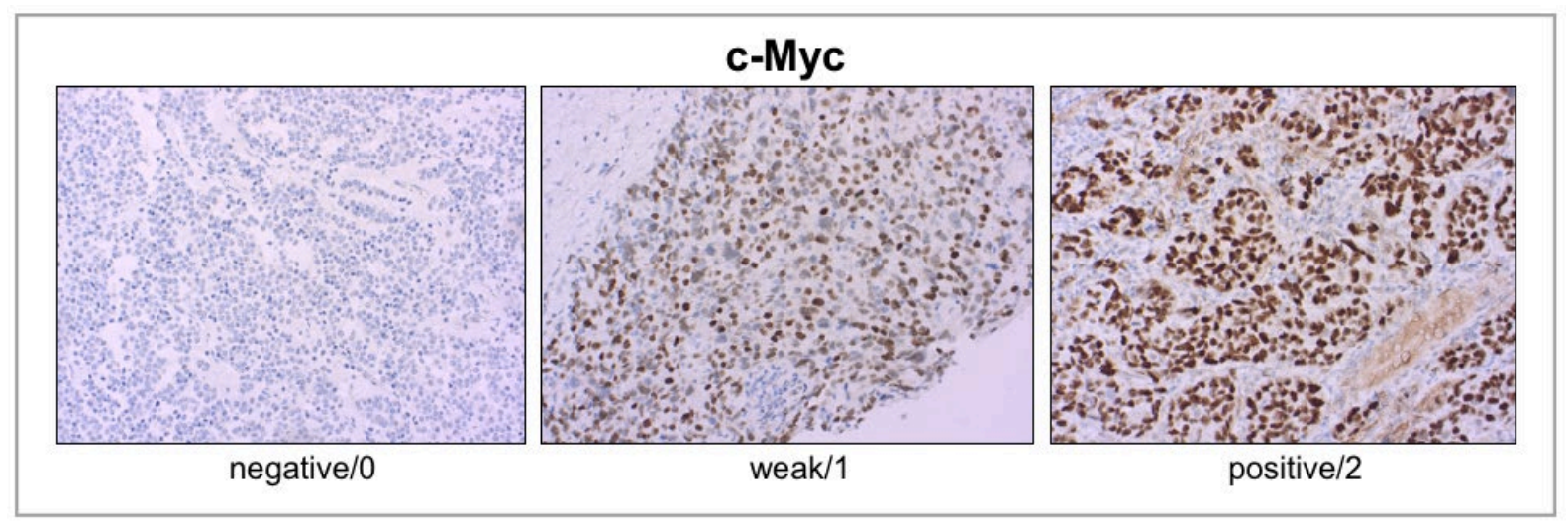

Figure 65: Immunohistochemistry staining intensity of c-Myc. Left: c-Myc negative tumor cells, AC, objective x20. Middle: c-Myc weak tumor cells, LCNEC, objective x20. Right: c-Myc positive tumor cells, LCNEC, objective $x 20$.

In all, nine cases (21\%) exhibited positive immunolabeling and 34 cases $(79 \%)$ exhibited negative immunolabeling for c-Myc. 2 TC (25\%), 1 AC (4\%), 6 LCNEC (75\%) and 0 SCC (0\%) showed positive C-Myc expression (Fig. 66). Of the eight LCNEC that were stained with c-Myc antibodies, five were classified as NEC (LCNEC A, B, E, F, $\mathrm{H}$ in Table 3) and showed strong c-Myc expression. The LCNEC C, D, and $\mathrm{G}$ which were stained with c-Myc antibodies and classified at NET G3, showed negative expression in two cases and positive expression in one case. If NET G3 exist in TNET, c-Myc may be a useful marker to identify NEC. 


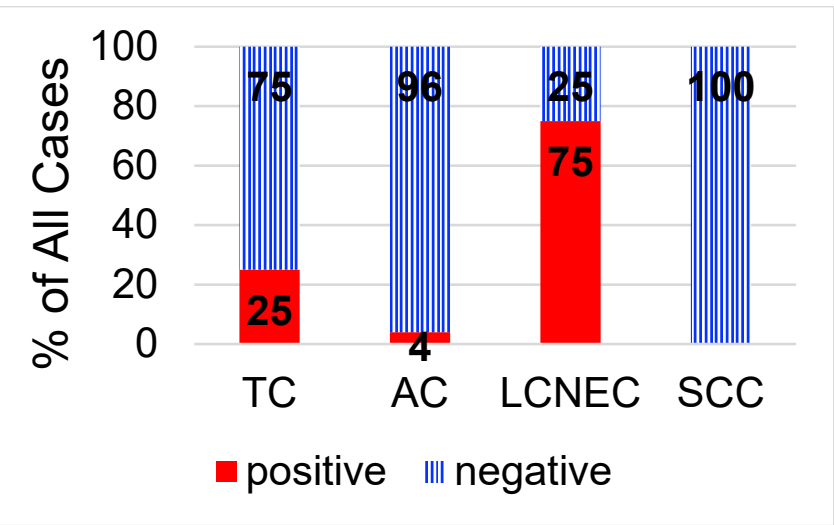

Figure 66: Distribution of c-Myc expression among WHO subgroups. The columns represent the percentage of cases with positive or negative antibody expression. Y-axis: percentage of stained cells, X-axis: WHO subgroup.

The Kaplan-Meier diagram depicts that c-Myc negative tumors were associated with longer survival and c-Myc positive tumors corresponded with dismal prognosis (Fig.67). The p-value was 0.07 in the log-rank test.

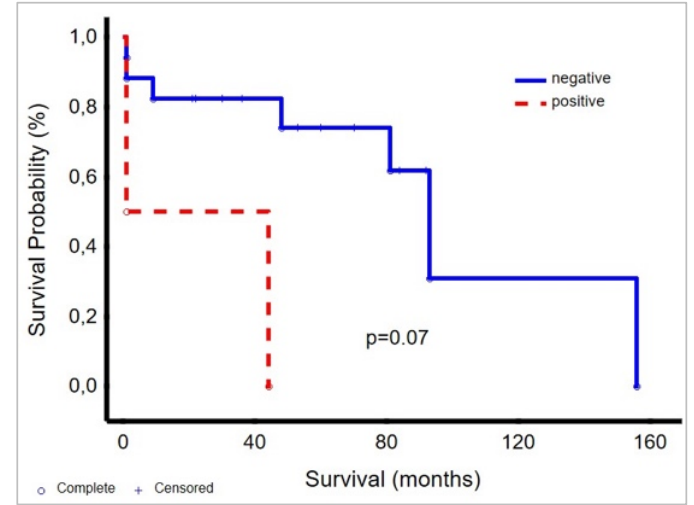

Figure 67: Survival analysis of TNET. The prognostic factor is two-tiered c-Myc expression. Y-axis: proportion surviving. $X$-axis: time in months.

Further, c-Myc exhibited a positive correlation with total chromosomal aberrations and the CNI (Fig. 68-69). Both parameters showed a higher range of genetic alterations with positive c-Myc expression than with negative c-Myc expression. For example, total chromosomal aberrations ranged from 25 to 50 for c-Myc negative tumors and from 60 to 80 for c-Myc positive tumors. The p-values were 0.01 and 0.003 for total chromosomal aberrations and CNI, respectively. Strong c-Myc expression corresponded with poor prognosis and higher genetic variations. 


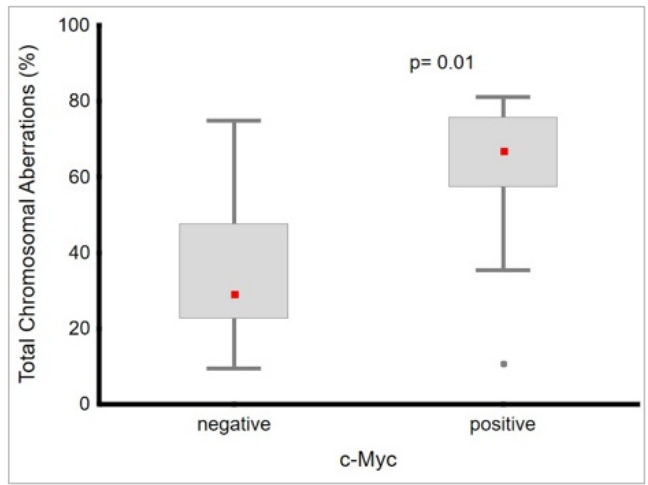

Figure 68: A correlation of c-Myc expression with total chromosomal aberrations. Y-axis: percentage of chromosomal aberrations. $X$ axis: negative or positive c-Myc staining.

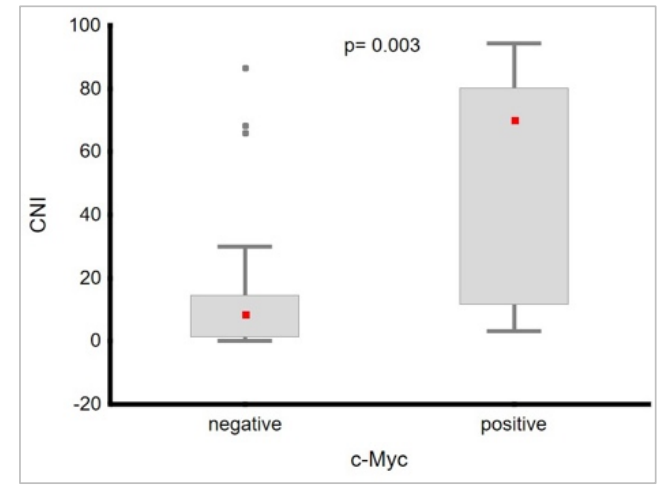

Figure 69: A correlation of c-Myc expression with CNI score. Y-axis: CNI score. X-axis: negative or positive c-Myc staining.

\subsubsection{SDHA Immunohistochemical Analysis}

SDHA staining was performed and assessed on 44 TNET (9 TC, 23 AC, 9 LCNEC, 3 SCC). The color intensity spectrum is shown in figure 70 . Cytoplasmic staining was observed in all cases.

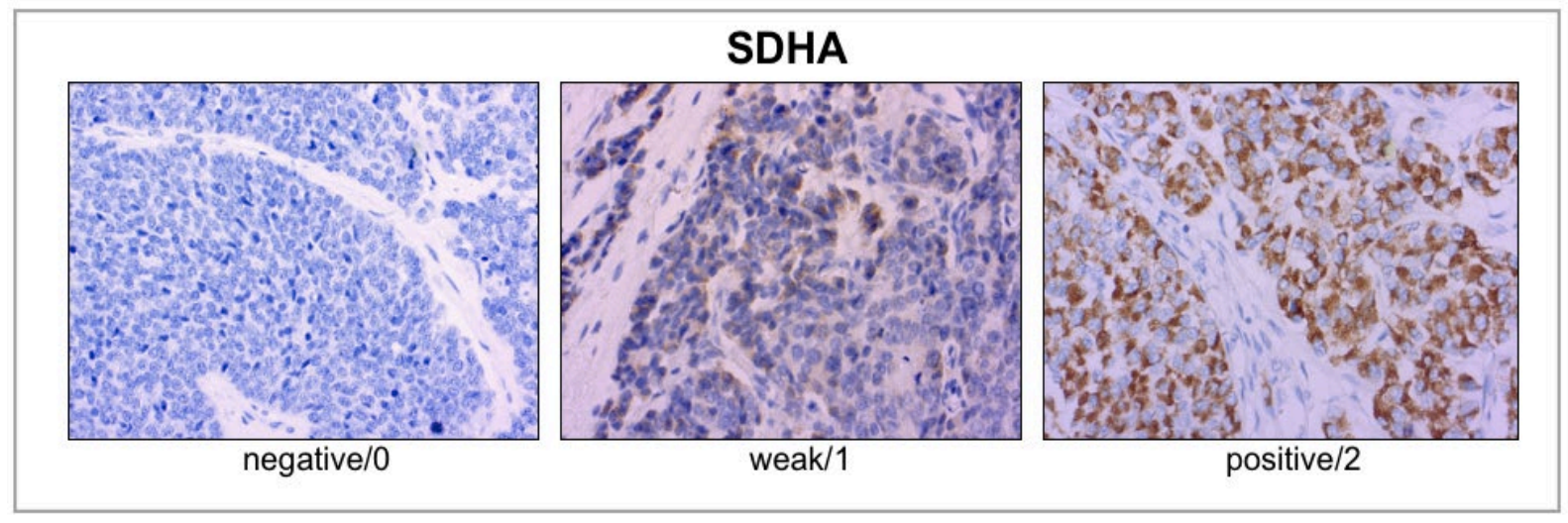

Figure 70: Immunohistochemistry staining intensity of SDHA. Left: SDHA negative tumor cells, AC, objective x40. Middle: SDHA weak tumor cells, AC, objective x40. Right: SDHA positive tumor cells, AC, objective x40.

In all, ten TNET (23\%) were positive and 34 TNET (77\%) were negative for SDHA expression. Interestingly, only tumors out of the carcinoid subgroups were positive. 4 out of 9 TC (44\%), 6 out of 23 AC (26\%), 0 out of 8 LCNEC $(0 \%)$ and 0 out of 3 SCC $(0 \%)$ showed positive SDHA protein expression (Fig. 71). 


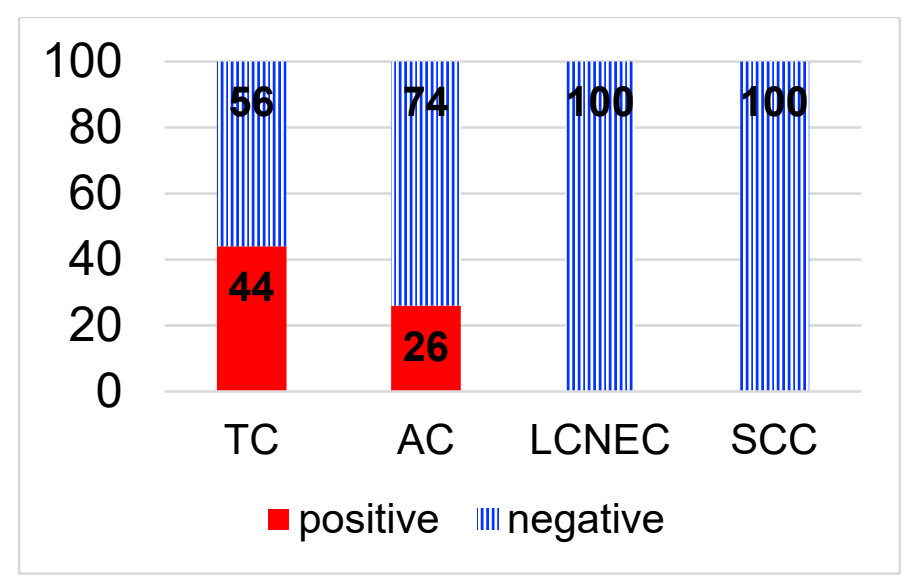

Figure 71: Distribution of SDHA expression among WHO subgroups. The columns represent the percentage of cases with positive or negative antibody expression. Y-axis: percentage of stained cells, X-axis: WHO subgroup.

Positive SDHA expression corresponded with a longer overall survival (Fig. 72). The positive curve remains at $100 \%$ survival probability until 150 months, then drops to $0 \%$ survival, due to an outlier in survival time. The negative curve shows a steady decline in survival probability with time. This finding was not statistically significant $(p=0.44)$.

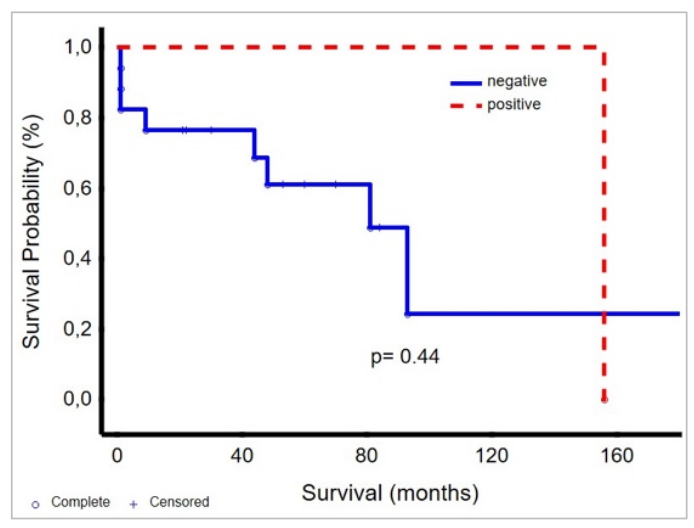

Figure 72: Survival analysis of TNET. The prognostic factor is two-tiered SDHA expression. Y-axis: proportion surviving. $X$-axis: time in months.

In the boxplots, SDHA negative tumors showed a greater and often higher range of chromosomal aberrations and CNI (Fig. 73-74), although negative and positive groups overlapped. There was no significant correlation between SDHA and genetic alterations. 


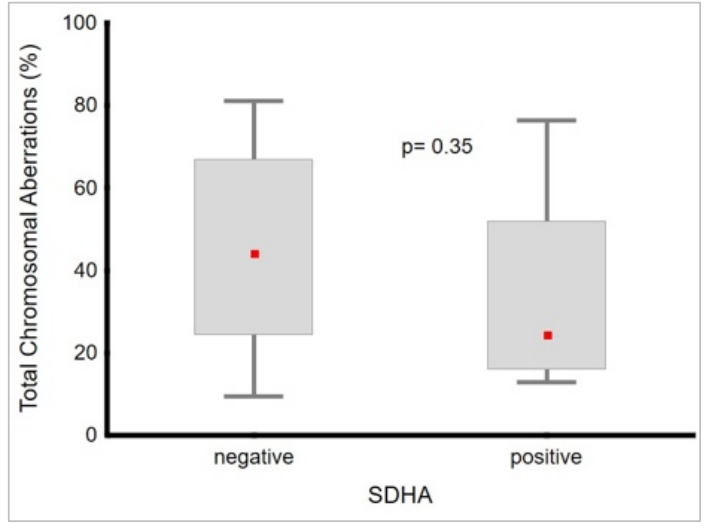

Figure 73: A correlation of SDHA expression with total chromosomal aberrations. Y-axis: percentage of chromosomal aberrations. X-axis: negative or positive SDHA staining.

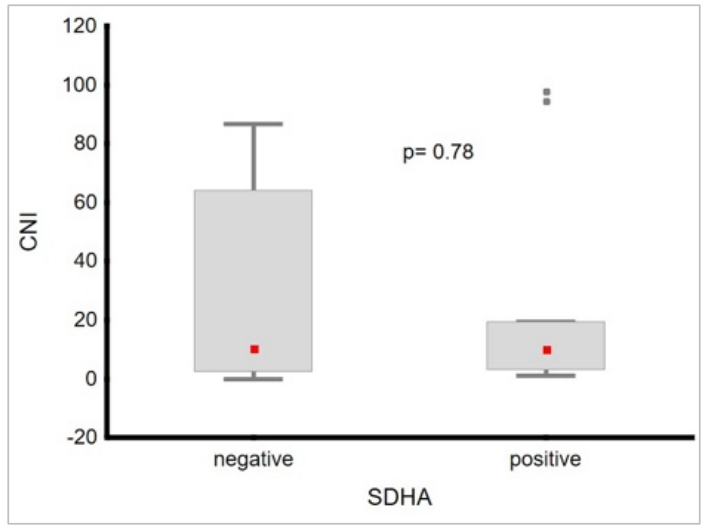

Figure 74: A correlation of SDHA expression with CNI score. Y-axis: CNI score. X-axis: negative or positive SDHA staining.

\subsubsection{SDHB Immunohistochemical Analysis}

44 TNET (10 TC, 23 AC, 8 LCNEC, 3 SCC) were evaluated after immunohistochemical staining with SDHB. The range of staining intensity can be seen in figure 75. Cytoplasmic staining was observed in all cases.

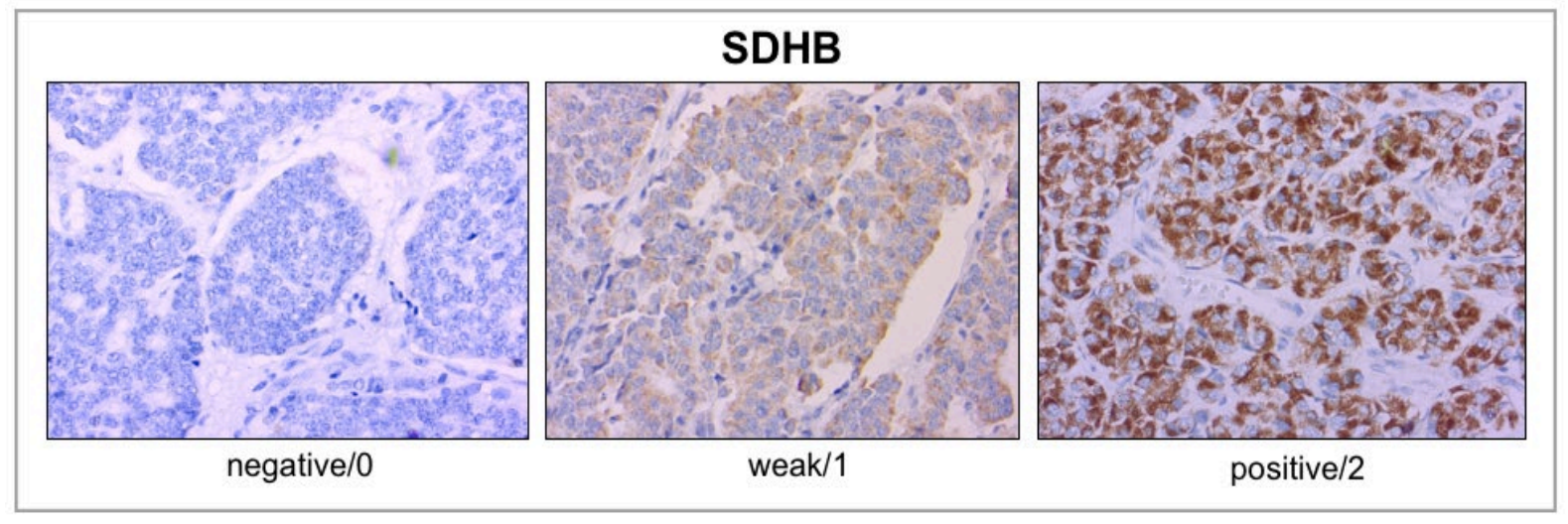

Figure 75: Immunohistochemistry staining intensity of SDHB. Left: SDHB negative tumor cells, AC, objective x40. Middle: SDHB weak tumor cells, LCNEC, objective x40. Right: SDHB positive tumor cells, AC, objective x40.

Figure 76 depicts the distribution of SDHB expression. In all, 17 TNET (43\%) showed positive immunolabeling and 27 TNET (67\%) showed negative immunolabeling. 4 of 10 TC (40\%), 7 of 23 AC (30\%), 5 of 8 LCNEC (62\%) and 1 of 3 SCC (33\%) expressed SDHB. In contrast to SDHA, SDHB positive expression was found in both carcinoids and carcinoma. In a three-tiered expression, 25 TNET (6 TC, 14 AC, 3 LCNEC, 2 SCC) showed negative, 7 TNET (6 AC, 1 SCC) showed weak and 12 TNET (4 TC, 3 AC, 5 LCNEC) showed strong SDHB immunolabeling. 


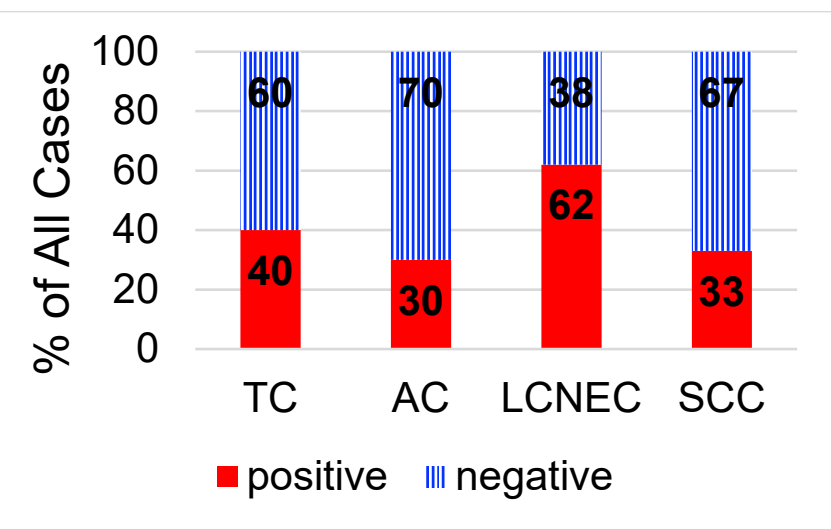

Figure 76: Distribution of SDHB expression among WHO subgroups. The columns represent the percentage of cases with positive or negative antibody expression. Y-axis: percentage of stained cells, X-axis: WHO subgroup.

In the survival analysis in figure 77 , the SDHB negative curve exhibits a steady decline of survival probability over time. In this cohort, SDHB did not correlate significantly with survival $(p=0.48)$.

The Kaplan-Meier diagram of a three-tiered SDHB expression illustrates the positive curve with longest survival probability, followed by negative and then weak expression curves (Fig. 78). Still, there was no significant discernment of survival between groups $(p=0.57)$.

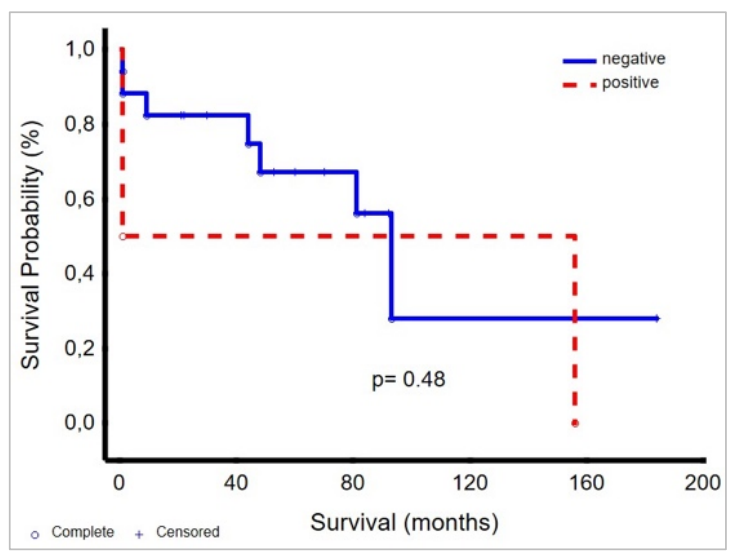

Figure 77: Survival analysis of TNET. The prognostic factor is two-tiered SDHB expression. $Y$ axis: proportion surviving. $X$-axis: time in months.

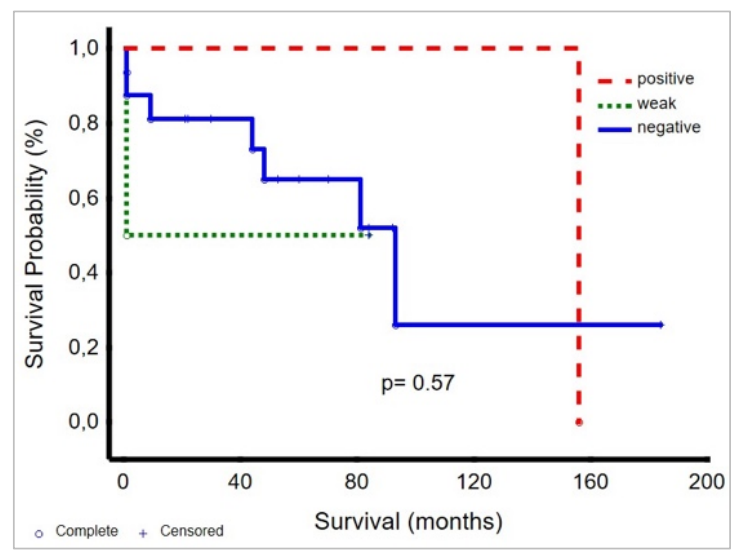

Figure 78: Survival Analysis of TNET. The prognostic factor is the three-tiered SDHB expression. Y-axis: proportion surviving. $X$-axis: time in months.

There was no significant correlation between SDHB expression and genetic aberrations (Fig. 79-80). Despite this, the values in SDHB positive tumors exhibited a greater range than SDHB negative tumors. 


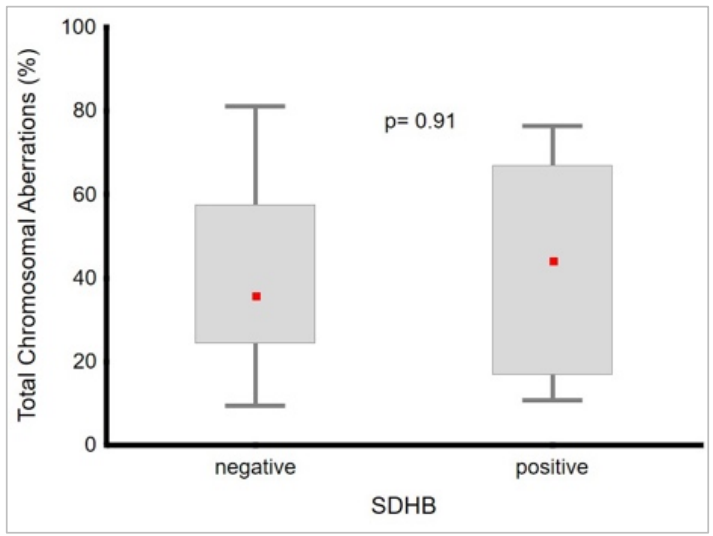

Figure 79: A correlation of SDHB expression with total chromosomal aberrations. $Y$-axis: percentage of chromosomal aberrations. $X$ axis: negative or positive SDHB staining.

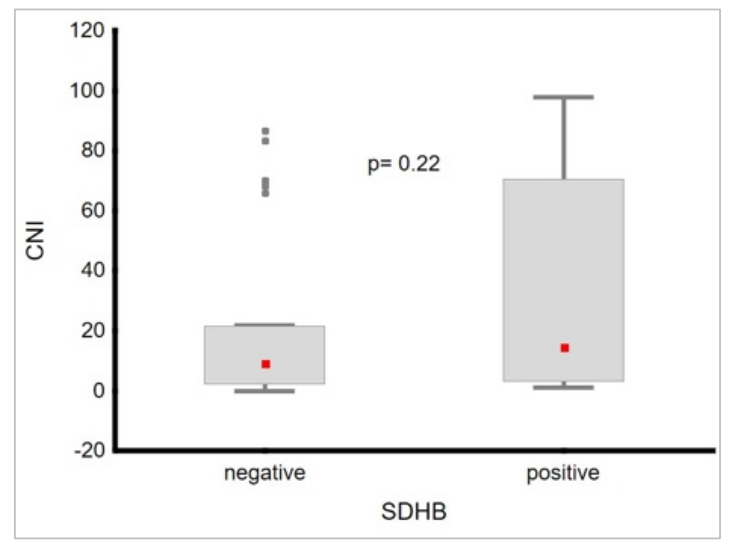

Figure 80: A correlation of SDHB expression with CNI score. Y-axis: CNI score. X-axis: negative or positive SDHB staining.

\subsubsection{EZH2 Immunohistochemical Analysis}

46 TNET (9 TC, 23 AC, 11 LCNEC, 3 SCC) were stained and evaluated for EZH2 protein expression. The spectrum of staining intensity levels is shown in figure 81 . EZH2 expression was found in the nucleus.

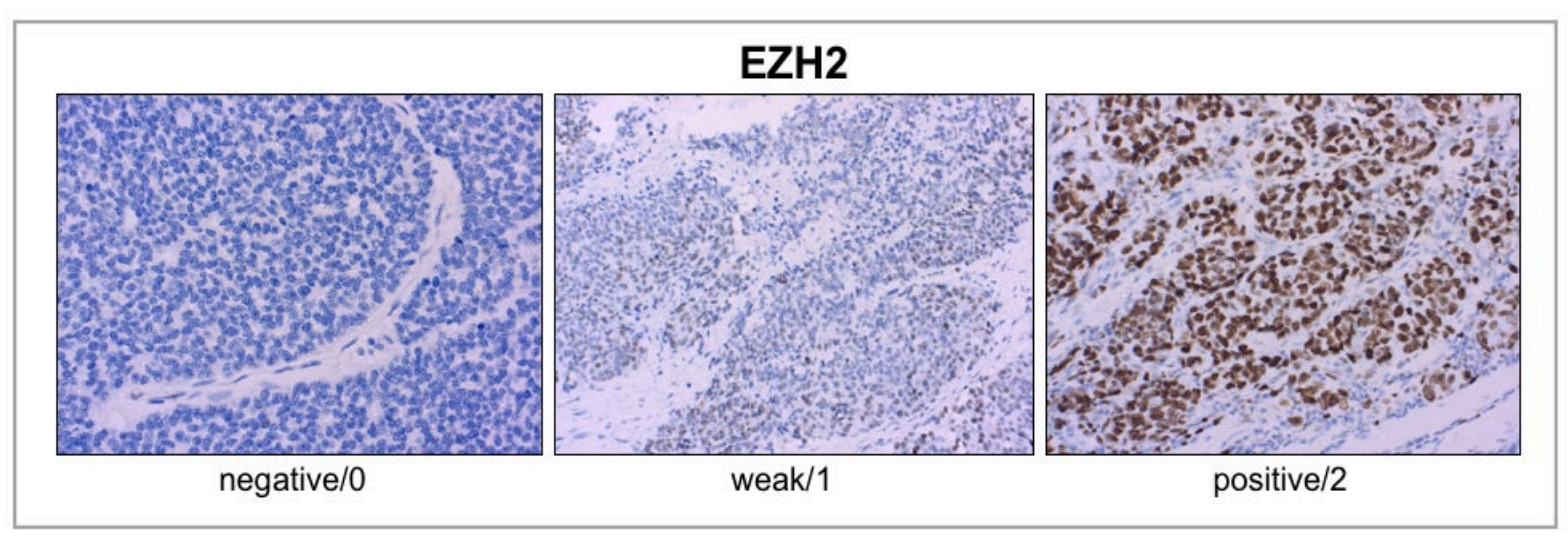

Figure 81: Immunohistochemistry staining intensity of EZH2. Left: EZH2 negative tumor cells, AC, objective x40. Middle: EZH2 weak tumor cells, SCC, objective x20. Right: EZH2 positive tumor cells, LCNEC, objective x20.

Positive immunolabeling was present in nine TNET (20\%) and negative immunolabeling was present in 37 TNET (80\%). 0 of 9 TC (0\%), 1 of $23 \mathrm{AC} \mathrm{(4 \% ),} 5$ of 11 LCNEC (45\%) and 3 of 3 SCC (100\%) showed positive protein expression (Fig. 82). As mentioned in chapter 4.5.3, EZH2 immunoreactivity was lost in all the LCNEC that were classified as NET G3. In comparison, the more poorly-differentiated LCNEC, with one exception, presented positive EZH2 immunoreactivity. In a three-tiered expression, 31 cases were negative (9 TC, 20 AC, 2 LCNEC), eight cases were weak (2 AC, 4 LCNEC, 2 SCC) and seven cases were positive (1 AC, 5 LCNEC, 1 SCC) for $\mathrm{EZH} 2$ expression. 


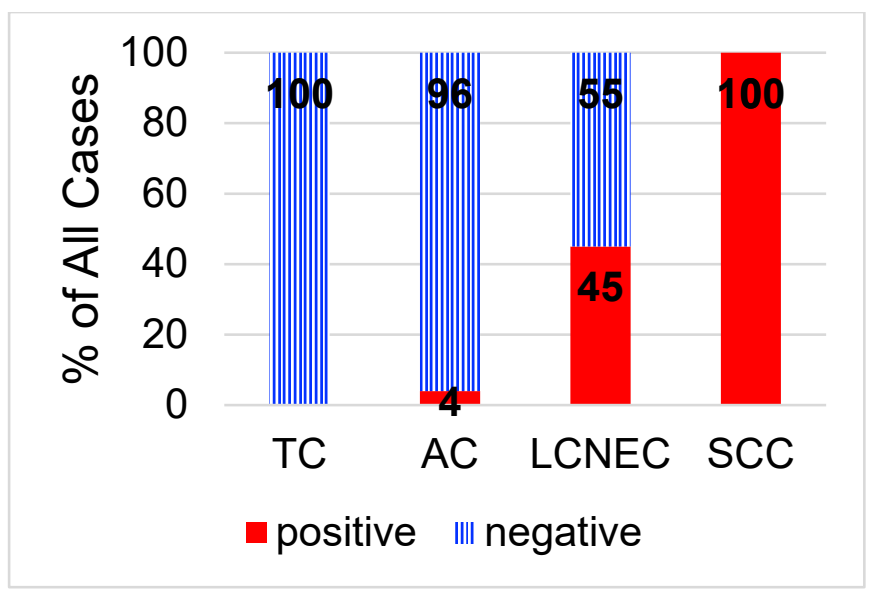

Figure 82: Distribution of EZH2 expression among WHO subgroups. The columns represent the percentage of cases with positive or negative antibody expression. Y-axis: percentage of stained cells, X-axis: WHO subgroup.

Survival curves of the TNET grouped into positive and negative expression, showed significant discernment in prognosis (Fig. 83). Tumors with negative EZH2 expression corresponded with longer survival than tumors with positive EZH2 expression $(p=$ 0.0002).

The three-tiered Kaplan-Meier curve in figure 84 shows a similar trend to figure 83 . $\mathrm{EZH} 2$ negative tumors associated with the longest survival. EZH2 weak tumors had shorter survival than negative tumors, but longer survival than the EZH2 positive tumors $(p=0.001)$. EZH2 positive tumors associated with the shortest survival.

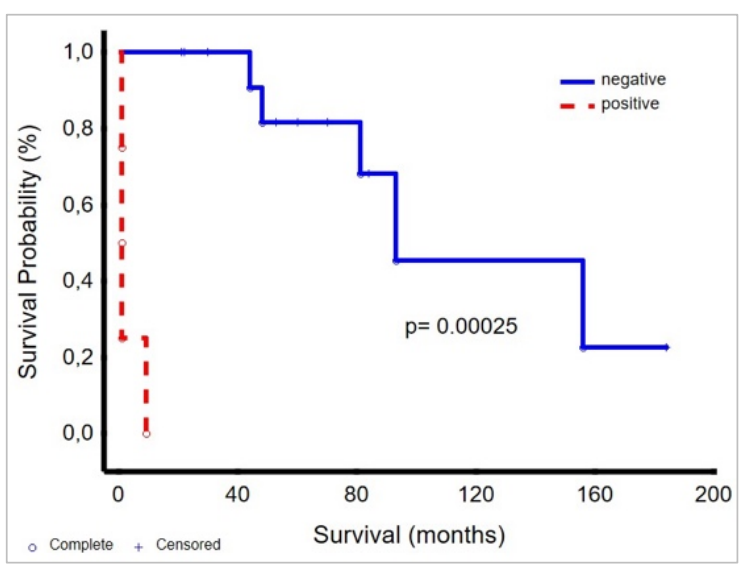

Figure 83: Survival analysis of TNET. The prognostic factor is two-tiered EZH2 expression. $Y$ axis: proportion surviving. $X$-axis: time in months.

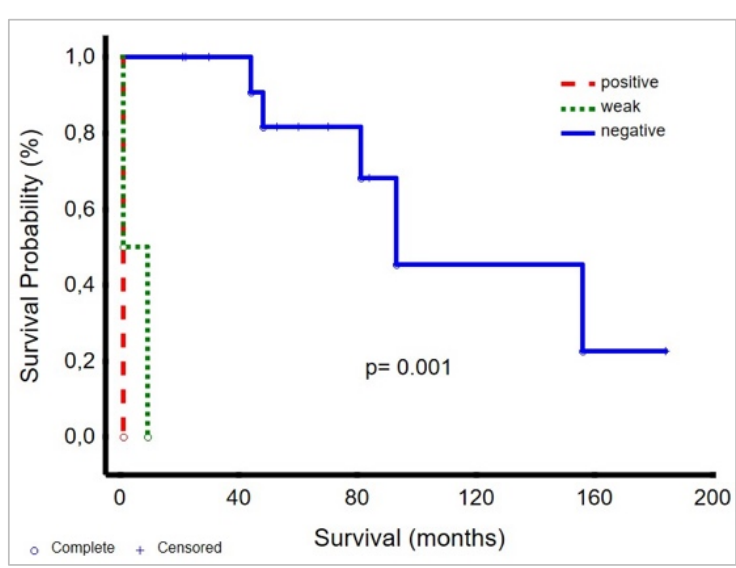

Figure 84: Survival Analysis of TNET. The prognostic factor is the three-tiered EZH2 expression. Y-axis: proportion surviving. $X$-axis: time in months.

The boxplots demonstrate a positive correlation between EZH2 expression and chromosomal aberrations and the CNI score (Fig. 85-86). Positive EZH2 expression corresponded with more chromosomal copy variations, while negative EZH2 expression corresponded with less copy variations. The p-values were 0.00 and 0.003 for chromosomal aberrations and $\mathrm{CNI}$ score, respectively. In all, the EZH2 
immunohistochemistry results correlated strongly with TNET subgroups, survival, and chromosomal number variants.

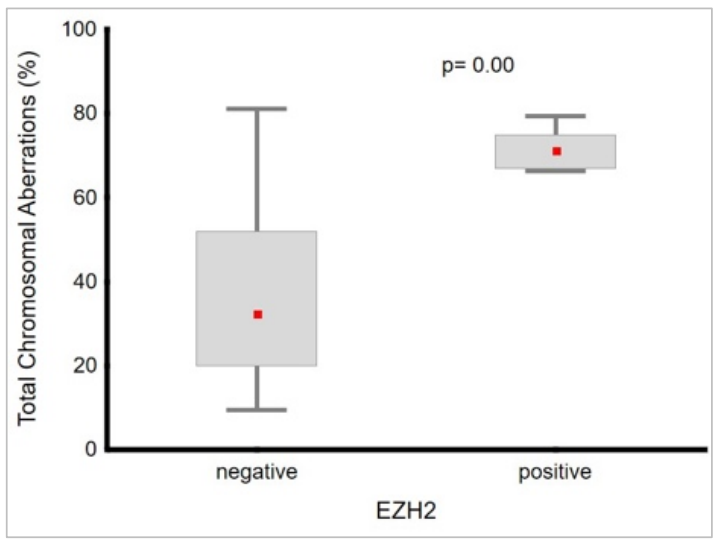

Figure 85: A correlation of EZH2 expression with total chromosomal aberrations. Y-axis: percentage of chromosomal aberrations. $X$-axis: negative or positive EZH2 staining.

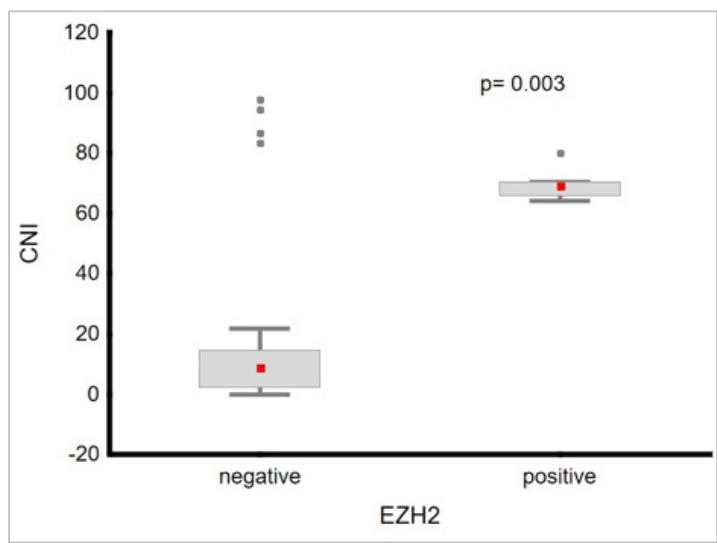

Figure 86: A correlation of EZH2 expression with CNI score. Y-axis: CNI score. X-axis: negative or positive EZH2 staining.

\subsubsection{H3K27me3 Immunohistochemical Analysis}

H3K27me3 was stained on 42 TNET (8 TC, 23 AC, 8 LCNEC, 3 SCC). Figure 87 shows examples of negative/0, weak/1, and strong/2 immunolabeling of the tumor cells. Again, the staining was nuclear within tumor cells.

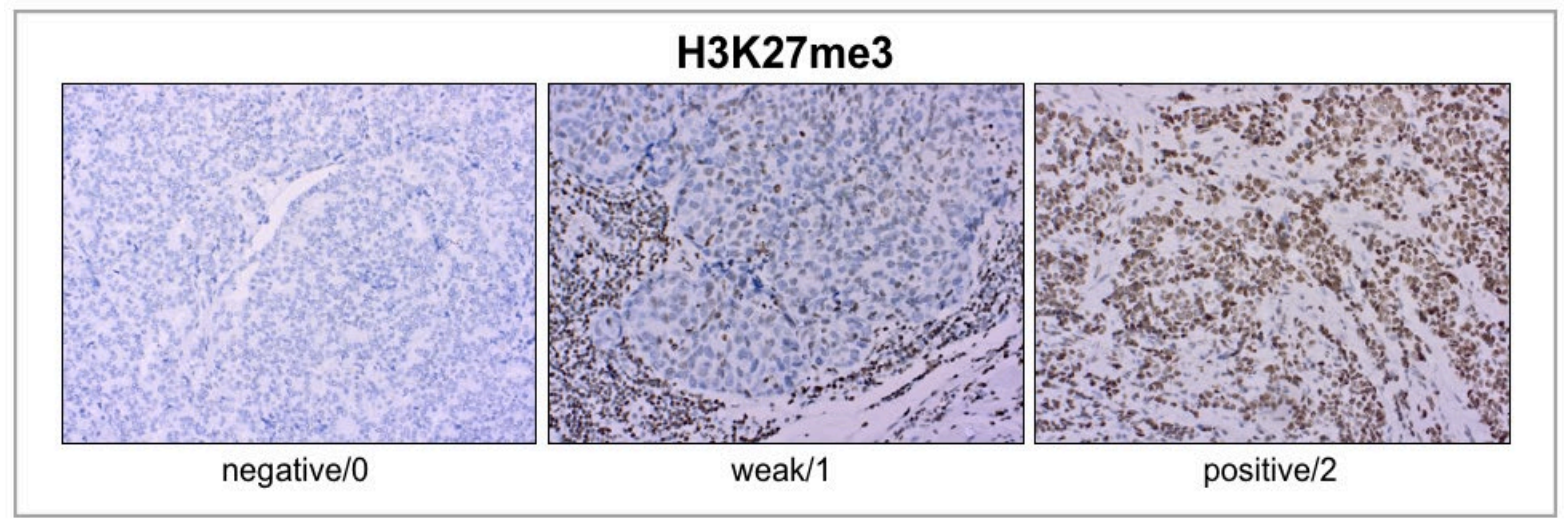

Figure 87: Immunohistochemistry staining intensity of H3K27me3. Left: H3K27me3 negative tumor cells, LCNEC, objective x20. Middle: H3K27me3 weak tumor cells, LCNEC, objective x2O. Right: H3K27me3 positive tumor cells, AC, objective $\times 20$.

Altogether, 20 cases exhibited positive and 22 cases exhibited negative H3K27me3 immunolabeling. Of the positive cases, 7 of 8 were TC (78\%), 9 of 23 were AC (39\%), 4 of 8 were LCNEC (50\%) and 0 of 3 were SCC (0\%) (Fig. 88). Interestingly H3K27me3 was found in almost all TC and was absent in SCC, while EZH2 was absent in TC and found in all SCC. In a three-tiered expression, 21 TNET showed 
negative (1 TC, 13 AC, 4 LCNEC, 3 SCC), 8 TNET showed weak (4 TC, 3 AC, 1 LCNEC) and 13 showed positive (3 TC, 7 AC, 3 LCNEC) immunolabeling.

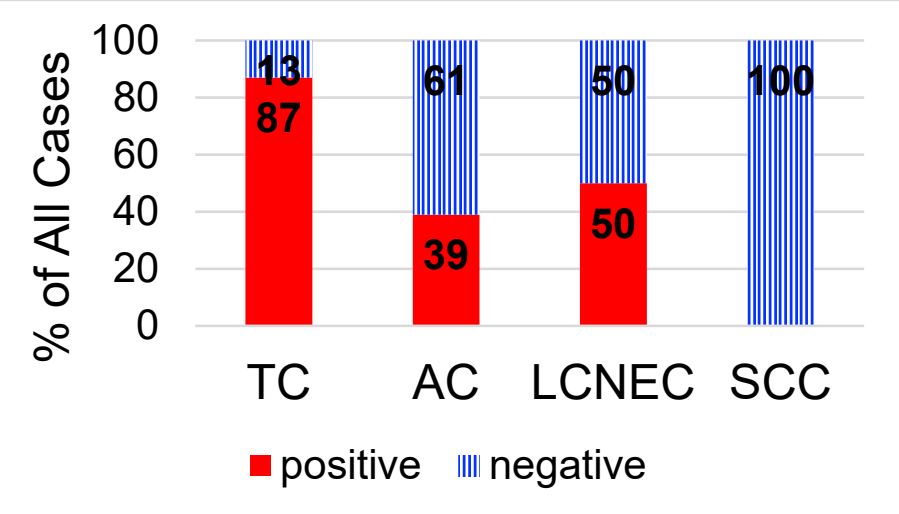

Figure 88: Distribution of H3K27me3 expression among WHO subgroups. The columns represent the percentage of cases with positive or negative antibody expression. Y-axis: percentage of stained cells, X-axis: WHO subgroup.

The Kaplan-Meier curve in figure 89 presents tumors with positive staining to associate with longer survival than tumors with negative staining. This correlation was however, not statistically significant $(p=0.31)$. In figure 90 , there is no significant correlation between survival and IHC expression.

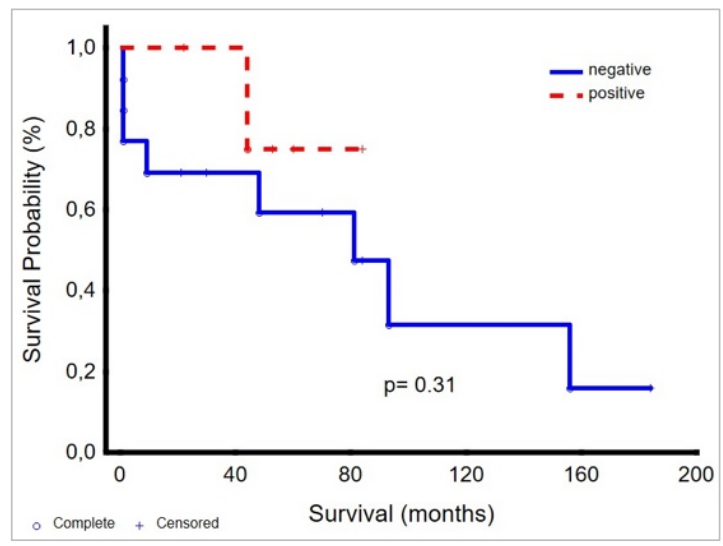

Figure 89: Survival analysis of TNET. The prognostic factor is two-tiered H3K27me3 expression. Y-axis: proportion surviving. X-axis: time in months.

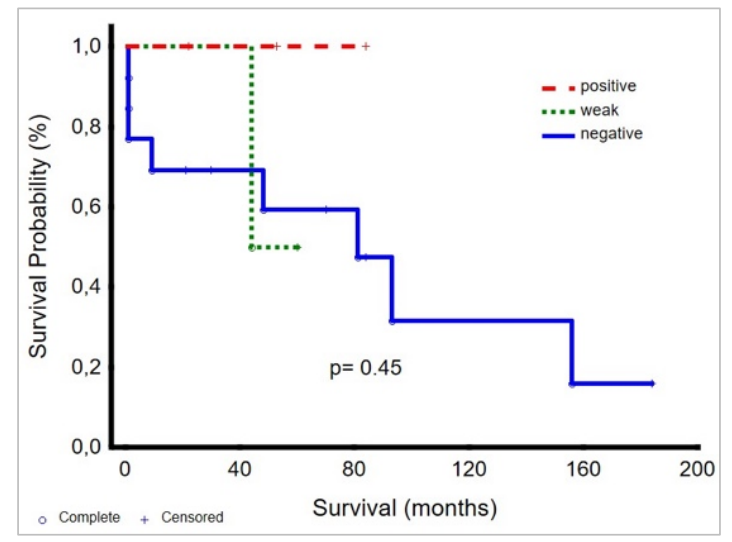

Figure 90: Survival Analysis of TNET. The prognostic factor is the three-tiered H3K27me3 expression. $Y$ axis: proportion surviving. $X$-axis: time in months.

H3K27me3 expression did not correlate significantly with total chromosomal aberrations, nor with the CNI score (Fig. 91-92). Despite this, the values in H3K27me3 positive tumors exhibited a greater range, and often higher values, than H3K27me3 negative tumors. 


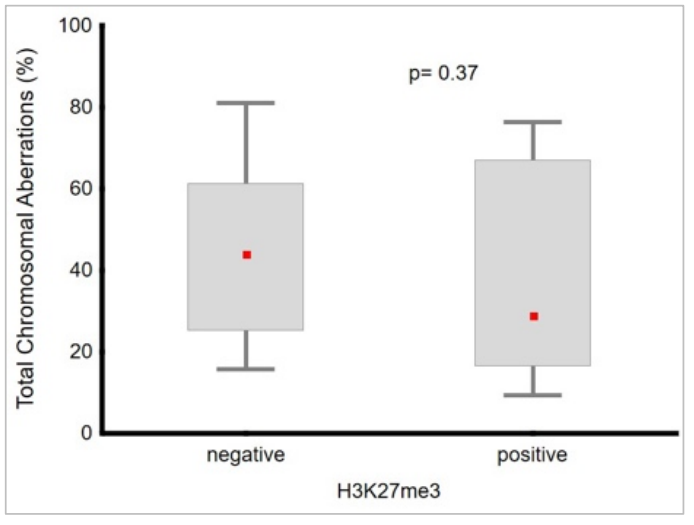

Figure 91: A correlation of H3K27me3 expression with total chromosomal aberrations. Y-axis: percentage of chromosomal aberrations. X-axis: negative or positive H3K27me3 staining.

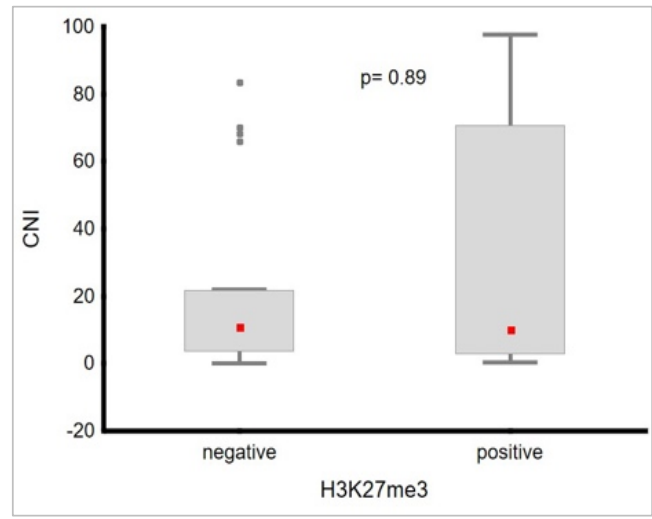

Figure 92: A correlation of H3K27me3 expression with CNI score. $Y$-axis: $C N I$ score. $X$ axis: negative or positive H3K27me3 staining.

\subsubsection{2 $\gamma \mathrm{H} 2 \mathrm{AX}$ Immunohistochemical Analysis}

$\gamma \mathrm{H} 2 \mathrm{AX}$ was stained in 44 TNET (10 TC, 23 AC, 8 LCNEC, 3 SCC). Figure 93 shows the spectrum of the antibody color intensity. $\gamma \mathrm{H} 2 \mathrm{AX}$ antibody demonstrated nuclear and cytoplasmic staining.

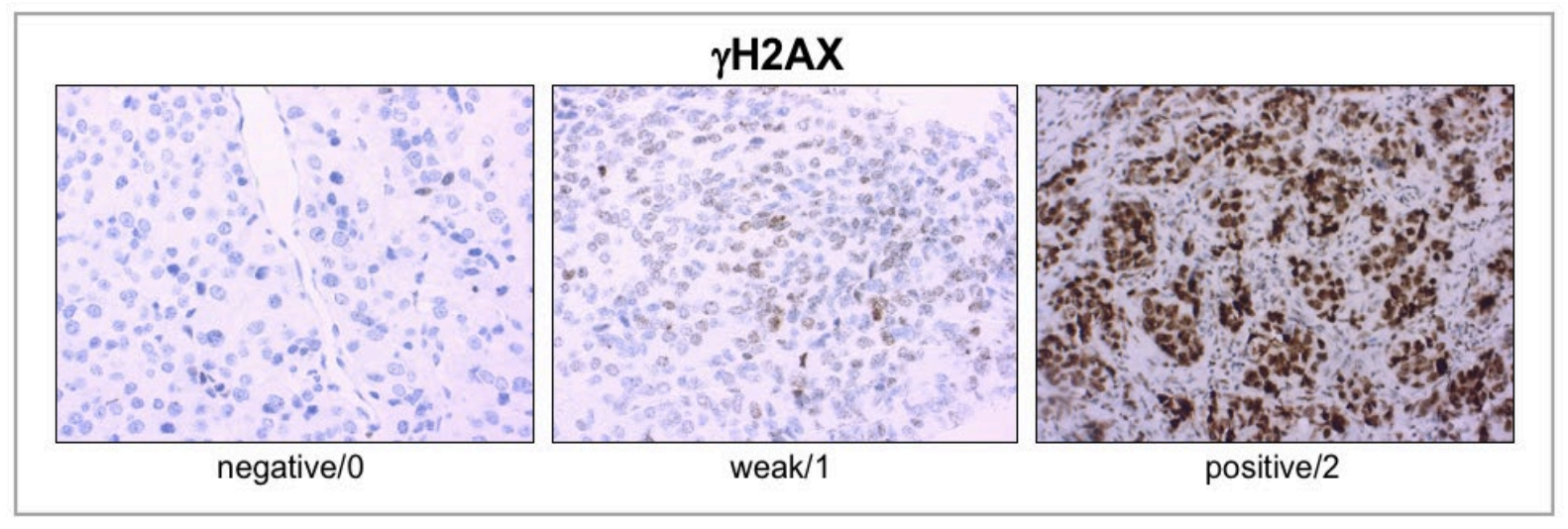

Figure 93: Immunohistochemistry stain intensity of $\gamma H 2 A X$. Left: $\gamma H 2 A X$ negative tumor cells, LCNEC, objective x40. Middle: $\gamma H 2 A X$ weak tumor cells, AC, objective x40. Right: $\gamma H 2 A X$ positive tumor cells, LCNEC, objective x40.

In all, 17 TNET displayed positive staining and 27 displayed negative staining of the antibody. 4 of 10 TC (40\%), 6 of 23 AC (26\%), 5 of 8 LCNEC (62\%) and 2 of 3 SCC $(67 \%)$ presented with positive immunolabeling (Fig. 94). Using a three-tiered staining evaluation, 22 TNET were negative (5 TC, 15 AC, 2 LCNEC), seven were weak (1 TC, 3 AC, 1 LCNEC, 2 SCC) and 15 were strong (4 TC, 5 AC, 5 LCNEC, 1 SCC). 


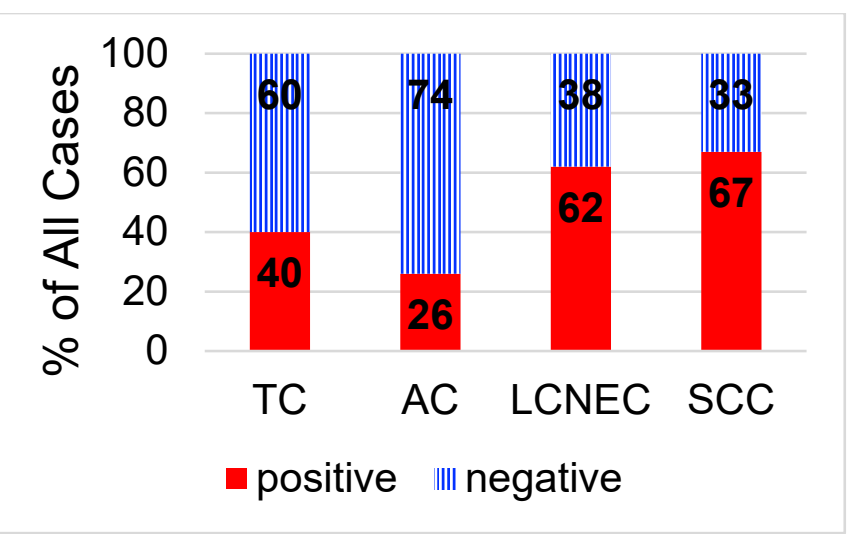

Figure 94: Distribution of $\mathrm{YH} 2 \mathrm{AX}$ expression among WHO subgroups. The columns represent the percentage of cases with positive or negative antibody expression. Y-axis: percentage of stained cells, X-axis: WHO subgroup.

No significant discernment between the $\gamma \mathrm{H} 2 \mathrm{AX}$-positive and -negative survival curves was found (Fig. 95). Overall, the positive staining group associated with longer survival. In the three-tiered Kaplan-Meier diagram (Fig. 96) weak staining tumors associated with the shortest overall survival. Negative staining tumors associated with the highest survival probability in the first 90 months. Positive staining tumors associated with the highest survival probability after $\geq 90$ months in this cohort. The difference between groups proved to be significant in the log-rank test $(p=0.02)$.

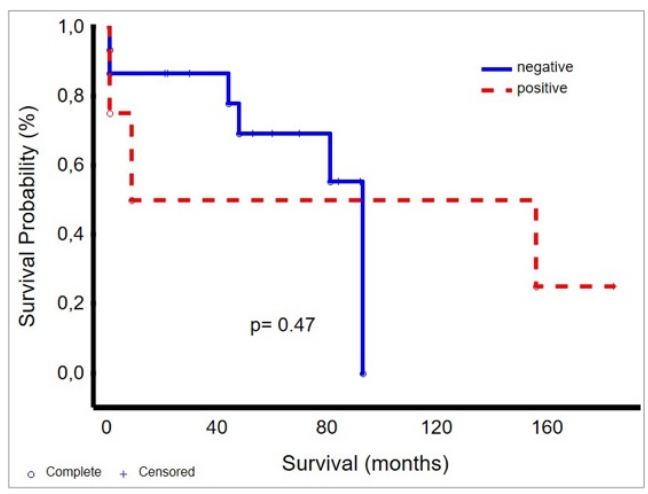

Figure 95: Survival analysis of TNET. The prognostic factor is two-tiered $\mathrm{HH} 2 \mathrm{AX}$ expression. Y-axis: proportion surviving. $X$ axis: time in months.

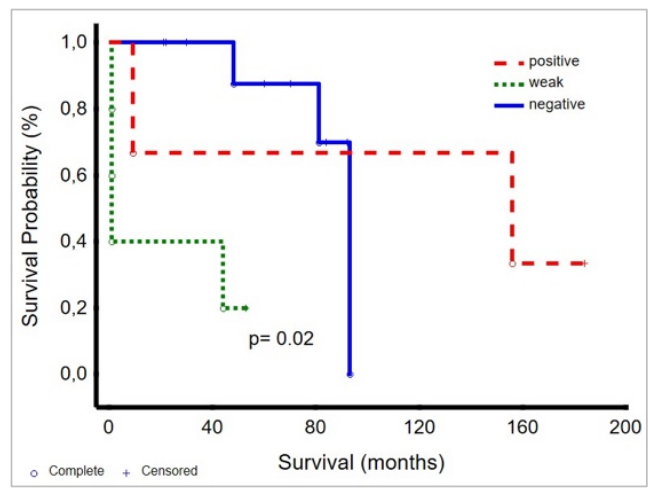

Figure 96: Survival Analysis of TNET. The prognostic factor is the three-tiered $\mathrm{\gamma H} 2 \mathrm{AX}$ expression. Y-axis: proportion surviving. $X$ axis: time in months.

$\gamma \mathrm{H} 2 \mathrm{AX}$ also proved to correlate significantly with total chromosomal aberrations (Fig. 97). Tumors with negative staining possessed, on average, more chromosomal aberrations than tumors with positive staining $(p=0.04)$. However, the $\mathrm{CNI}$ score did not show significant correlation with $\gamma \mathrm{H} 2 \mathrm{AX}$ immunolabeling (Fig. 98). Nevertheless, both boxplots show a tendency of higher chromosomal number variations with positive $\gamma \mathrm{H} 2 \mathrm{AX}$ tumor staining. 


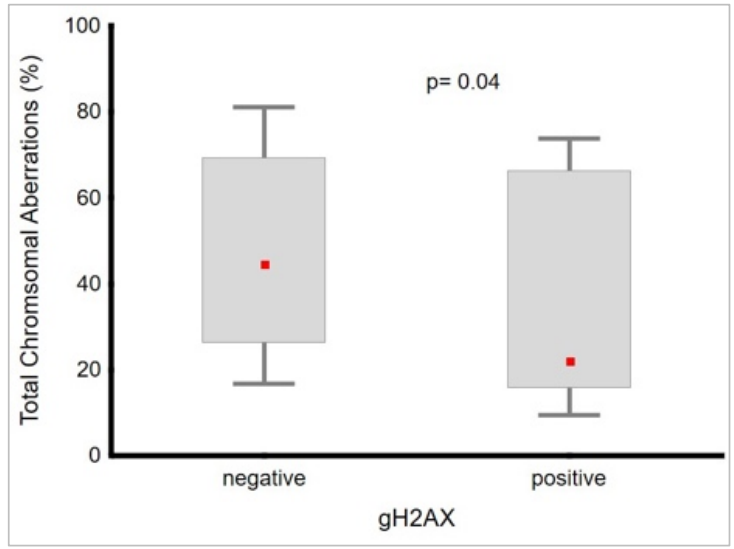

Figure 97: A correlation of $\gamma \mathrm{H} 2 \mathrm{AX}$ expression with total chromosomal aberrations. Y-axis: percentage of chromosomal aberrations. X-axis: negative or positive $\mathrm{\gamma H} 2 \mathrm{AX}$ staining.

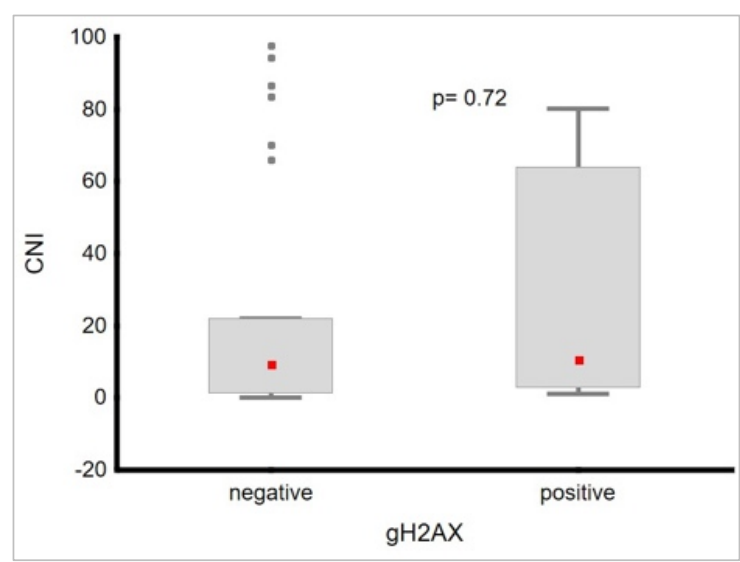

Figure 98: A correlation of $\gamma \mathrm{H} 2 \mathrm{AX}$ expression with CNI score. Y-axis: $C N I$ score. X-axis: negative or positive $\mathrm{\gamma H} 2 \mathrm{AX}$ staining.

\subsubsection{Chromogranin A Immunohistochemical Analysis}

49 TNET, consisting of 10 TC, 25 AC, 11 LCNEC, and 3 SCC were evaluated for chromogranin A immunolabeling. The TNET expression of Chromogranin A was only either lost (0) or very strong (2). The negative and positive protein expression are depicted in figure 99. Staining was found in the cytoplasm.

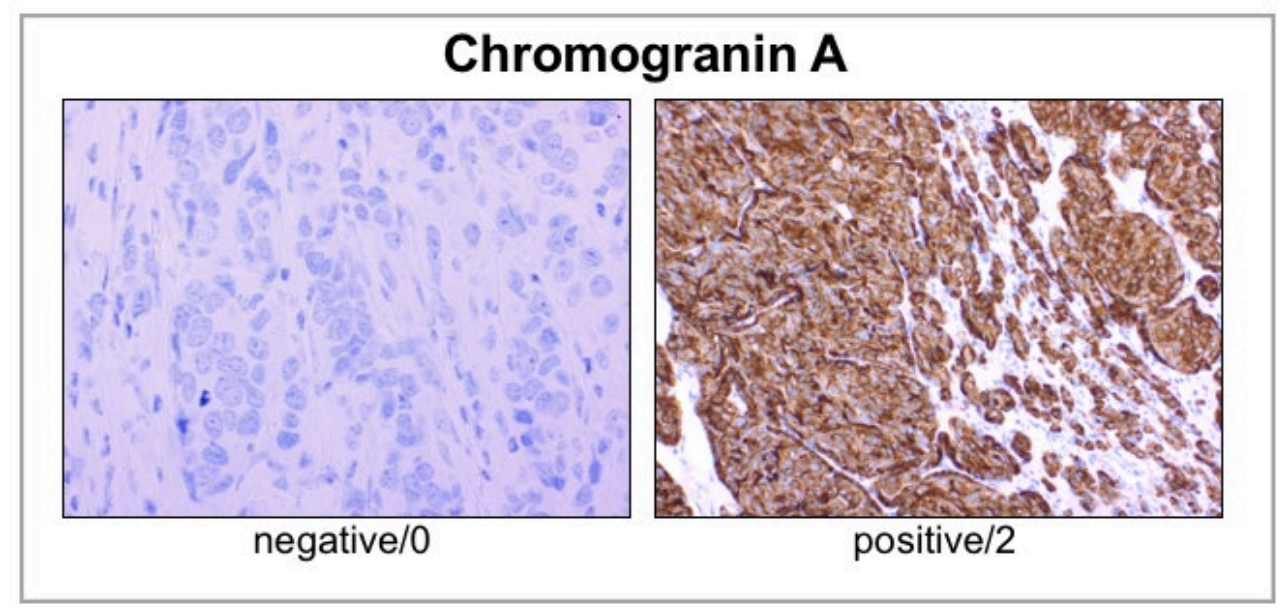

Figure 99: Immunohistochemistry staining intensity of chromogranin A. Left: chromogranin A negative tumor cells, LCNEC, objective x40. Right: chromogranin A positive tumor cells, AC, objective x40.

In all, 41 of the 49 TNET (84\%) exhibited positive protein expression. Eight cases $(16 \%)$ exhibited negative protein expression. More precisely, 10 of 10 TC (100\%), 24 of 24 AC (100\%), 6 of 8 LCNEC (75\%) and 0 of 3 SCC $(0 \%)$ exhibited positive expression (Fig. 100). The frequency of chromogranin A expression declined with higher tumor grade. 


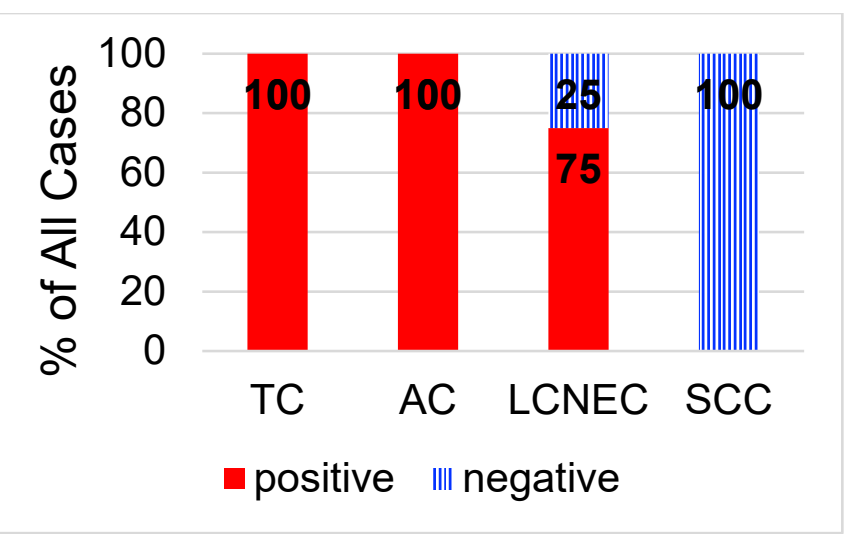

Figure 100: Distribution of chromogranin A expression among WHO subgroups. The columns represent the percentage of cases with positive or negative antibody expression. $Y$-axis: percentage of stained cells, X-axis: WHO subgroup.

The Kaplan-Meier diagram displays the survival of patients with chromogranin A positive and negative staining tumors (Fig. 101). Positive staining tumors showed longer overall survival. Negative staining tumors corresponded with dismal prognosis. The separation of these two groups proved to be significant $(p=0.0014)$.

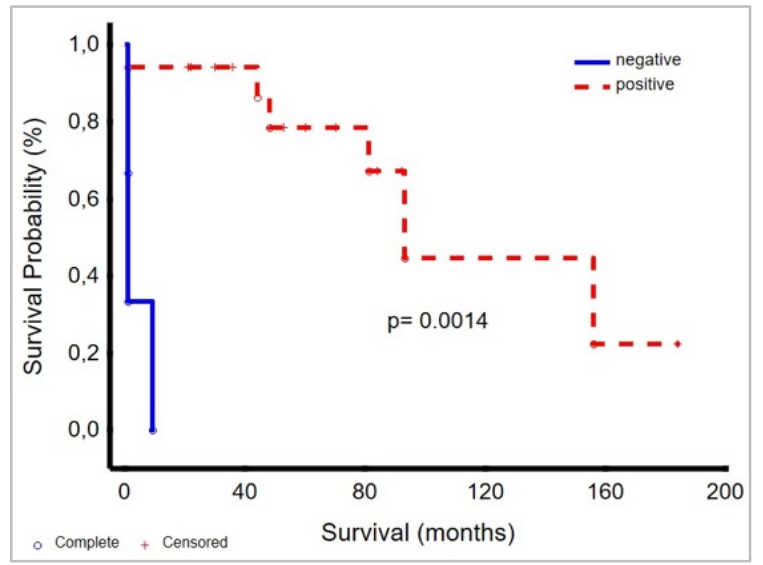

Figure 101: Survival analysis of TNET. The prognostic factor is two-tiered chromogranin A expression. Y-axis: proportion surviving. $X$-axis: time in months.

Additionally, Chromogranin A correlated significantly with total chromosomal aberrations and CNI score (Fig. 102-103). When chromogranin A was positive, there were less chromosomal aberrations and a lower CNI score. When chromogranin A was negative, higher values of both parameters were observed. The p-values were 0.0009 and 0.0008 for chromosomal aberrations and $\mathrm{CNI}$ score, respectively. 


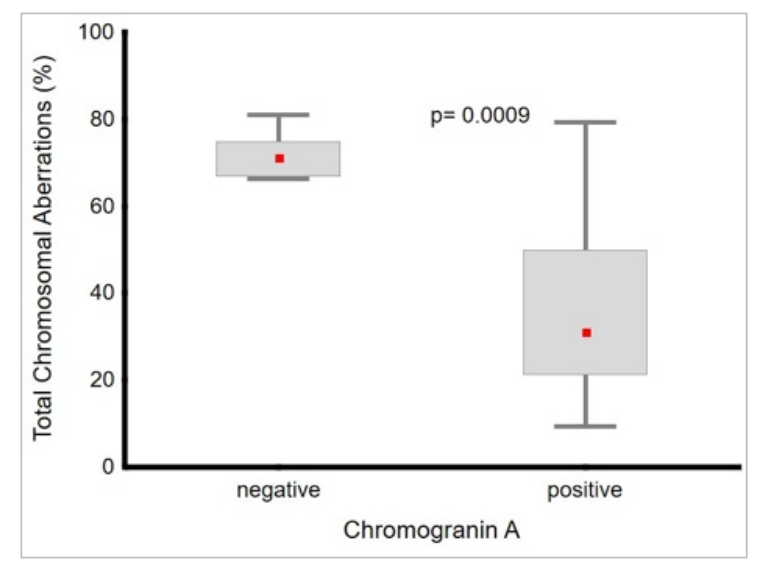

Figure 102: A correlation of chromogranin A expression with total chromosomal aberrations. $Y$ axis: percentage of chromosomal aberrations. Xaxis: negative or positive chromogranin A staining.

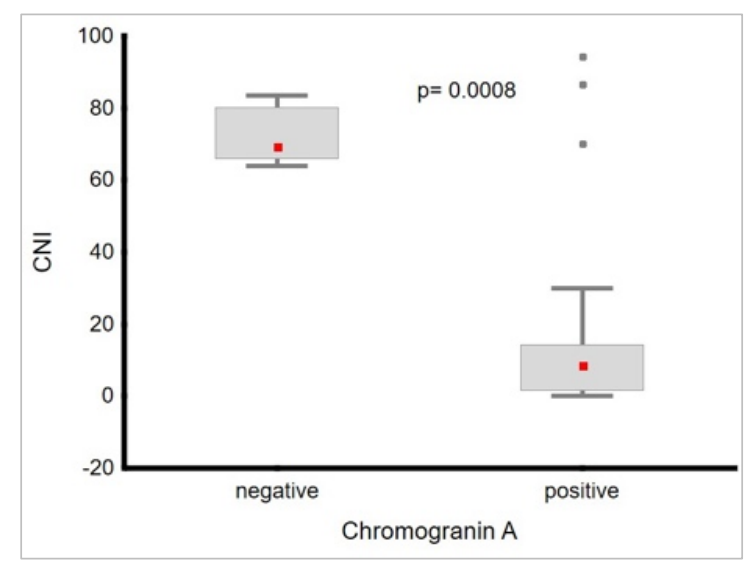

Figure 103: A correlation of chromogranin A expression with $\mathrm{CNI}$ score. Y-axis: CNI score. Xaxis: negative or positive chromogranin A staining.

\subsubsection{Keratin Immunohistochemical Analysis}

44 TNET (9 TC, 24 AC, 8 LCNEC, 3 SCC) were assessed for Keratin expression. Figure 104 shows the spectrum of antibody color intensity. Keratin staining was located in the cytoplasm.

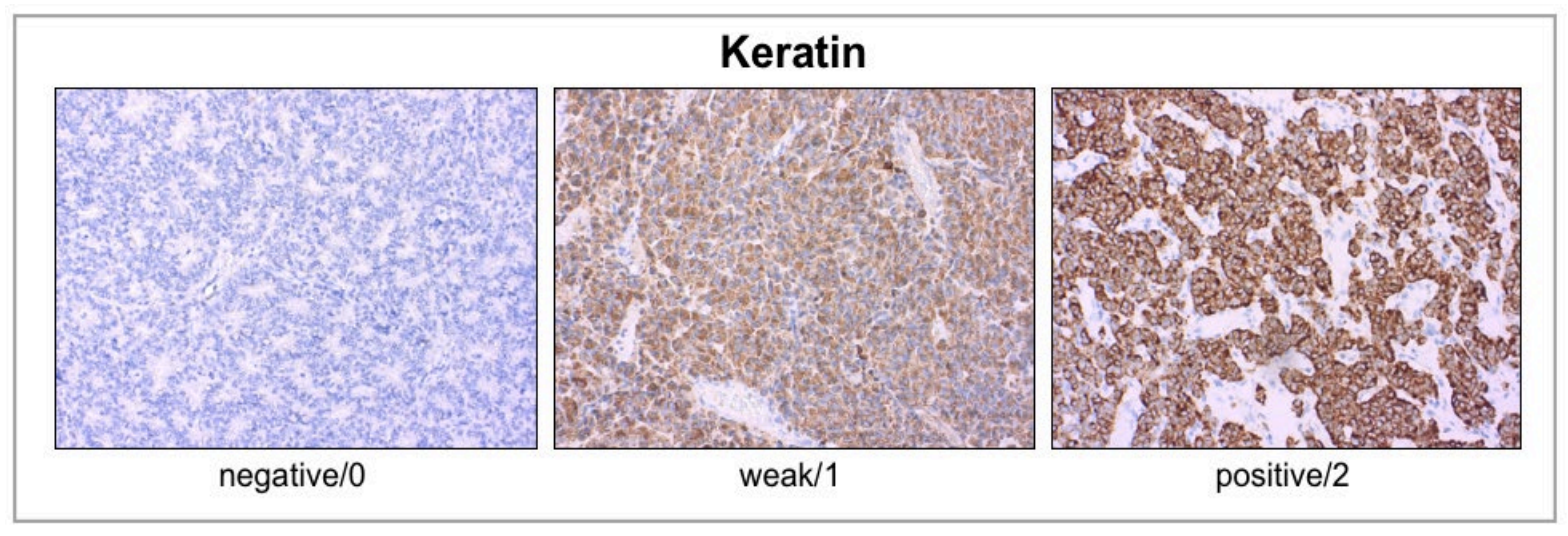

Figure 104: Immunohistochemistry staining intensity of Keratin. Left: keratin negative tumor cells, LCNEC, objective x20. Middle: keratin weak tumor cells, AC, objective x20. Right: keratin positive tumor cells, AC, objective $\times 20$.

36 cases stained positive and 8 cases stained negative with keratin antibody. Specifically, 7 of 9 TC (78\%), 20 of 24 AC (83\%), 7 of 8 LCNEC (88\%) and 2 of 3 SCC $(67 \%)$ showed positive protein expression (Fig. 105). The occurrence of keratin expression remained almost constant with rising WHO tumor grade. 


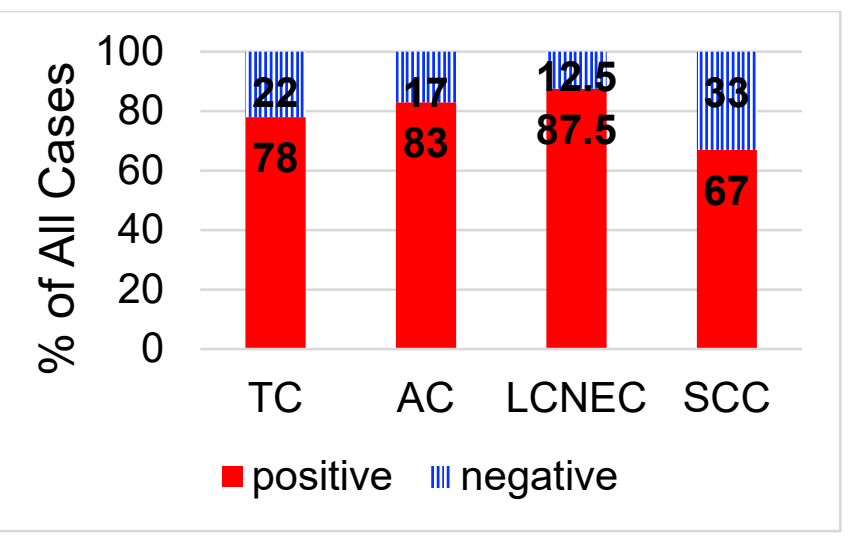

Figure 105: Distribution of keratin expression among WHO subgroups. The columns represent the percentage of cases with positive or negative antibody expression. Y-axis: percentage of stained cells, X-axis: WHO subgroup.

The survival analysis was not statistically significant. Positive and negative staining tumors did not show a strong differentiation in survival (Fig. 106).

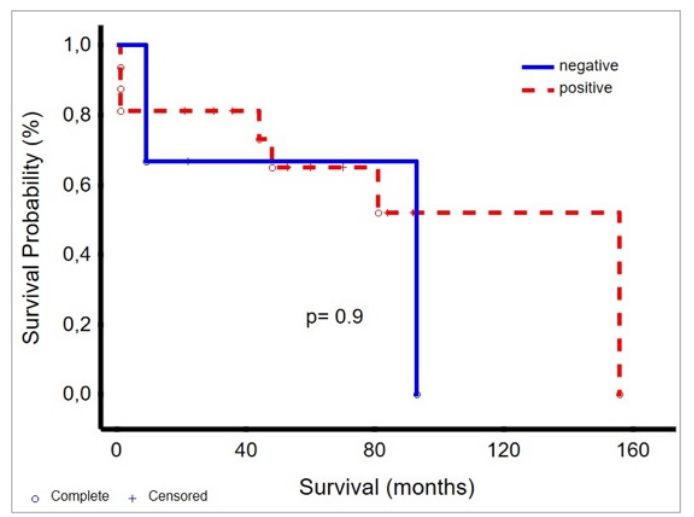

Figure 106: Survival analysis of TNET. The prognostic factor is two-tiered keratin expression. Y-axis: proportion surviving. $X$-axis: time in months.

Interestingly, keratin expression correlated significantly with total chromosomal aberrations $(p=0.02)$ (Fig.107). Positive keratin expression related to higher chromosomal aberrations than keratin negative tumors. The average number of chromosomal aberrations was about 45 for keratin positive tumors, and about 25 for keratin negative tumors. The CNI score did not confirm this correlation (Fig. 108). 


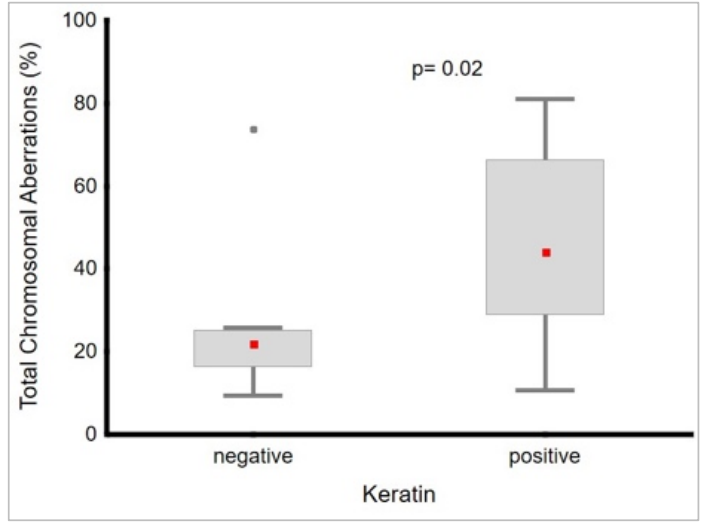

Figure 107: A correlation of keratin expression with total chromosomal aberrations. Y-axis: percentage of chromosomal aberrations. X-axis: negative or positive keratin staining.

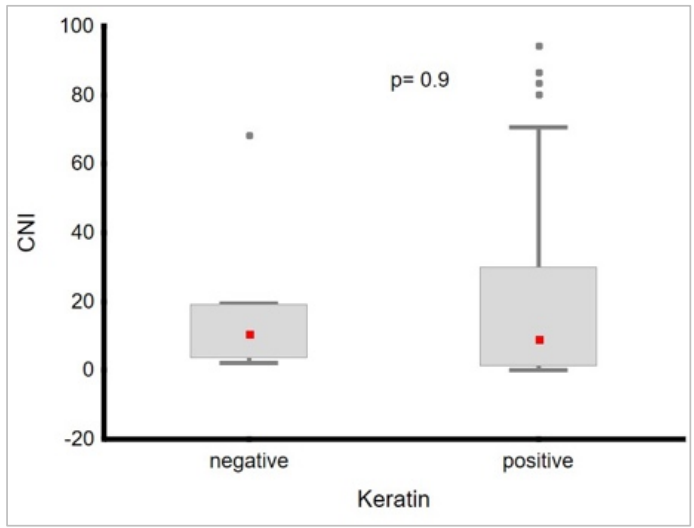

Figure 108: A correlation of keratin expression with CNI score. Y-axis: CNI score. X-axis: negative or positive keratin staining.

\subsubsection{Calcitonin Immunohistochemical Analysis}

45 TNET (9 TC, 24 AC, 9 LCNEC, 3 SCC) were assessed for calcitonin expression. The intensity spectrum for antibody staining is illustrated in figure 109. The expression was cytoplasmic.

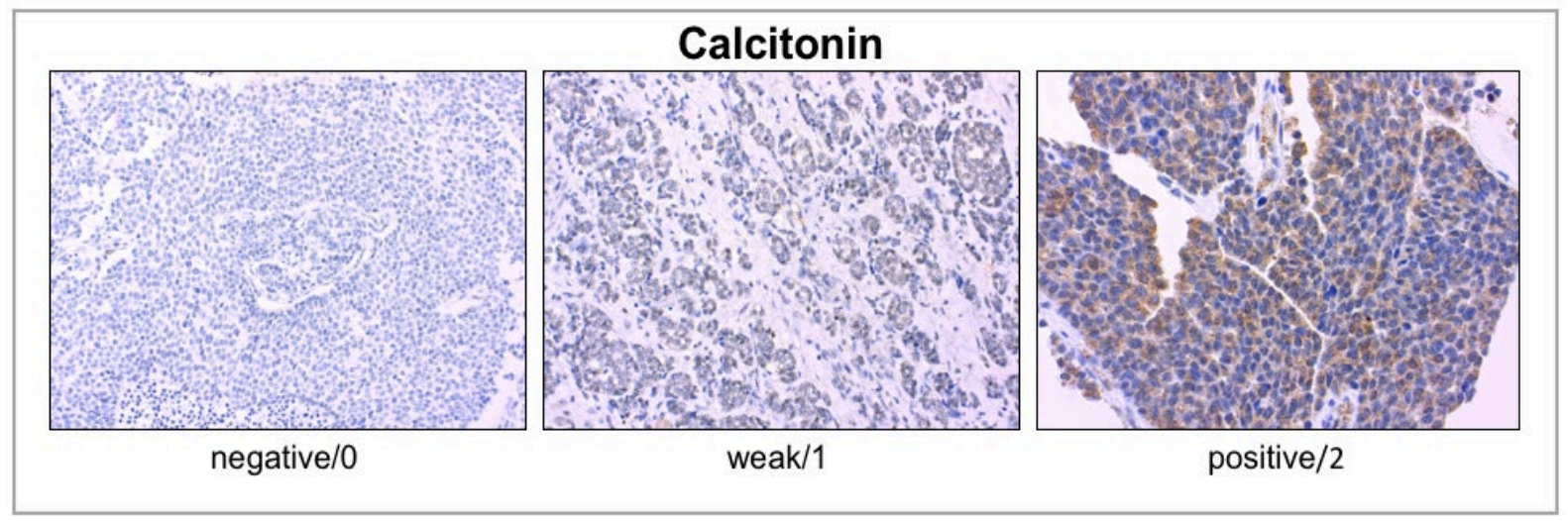

Figure 109: Immunohistochemistry staining intensity of calcitonin. Left: calcitonin negative tumor cells, $A C$, objective x20. Middle: calcitonin weak tumor cells, TC, objective x20. Left: calcitonin positive tumor cells, AC, objective $\times 40$.

Only two cases (1 TC and 1 AC) (0.4\%) expressed calcitonin, 43 cases (96\%) did not express calcitonin (Fig. 110). 


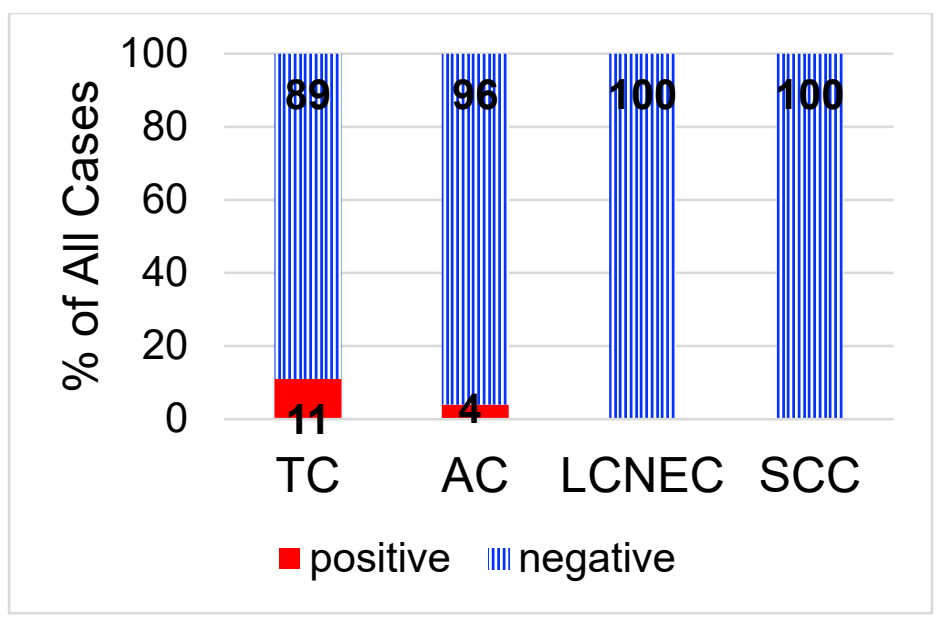

Figure 110: Distribution of calcitonin expression among WHO subgroups. The columns represent the percentage of cases with positive or negative antibody expression. Y-axis: percentage of stained cells, X-axis: WHO subgroup.

Creating two survival curves was not possible due to a lack of positive cases. The survival curve was the same as in chapter 4.7.4. There was no correlation between calcitonin staining and total chromosomal aberrations and $\mathrm{CNI}$ score.

\subsubsection{YAP1 Immunohistochemical Analysis}

44 TNET (10 TC, 23 AC, 8 LCNEC, 3 SCC) were stained with YAP1 antibody. The range of staining intensity is shown in figure 111. The antibody expression accumulated within the cytoplasm.

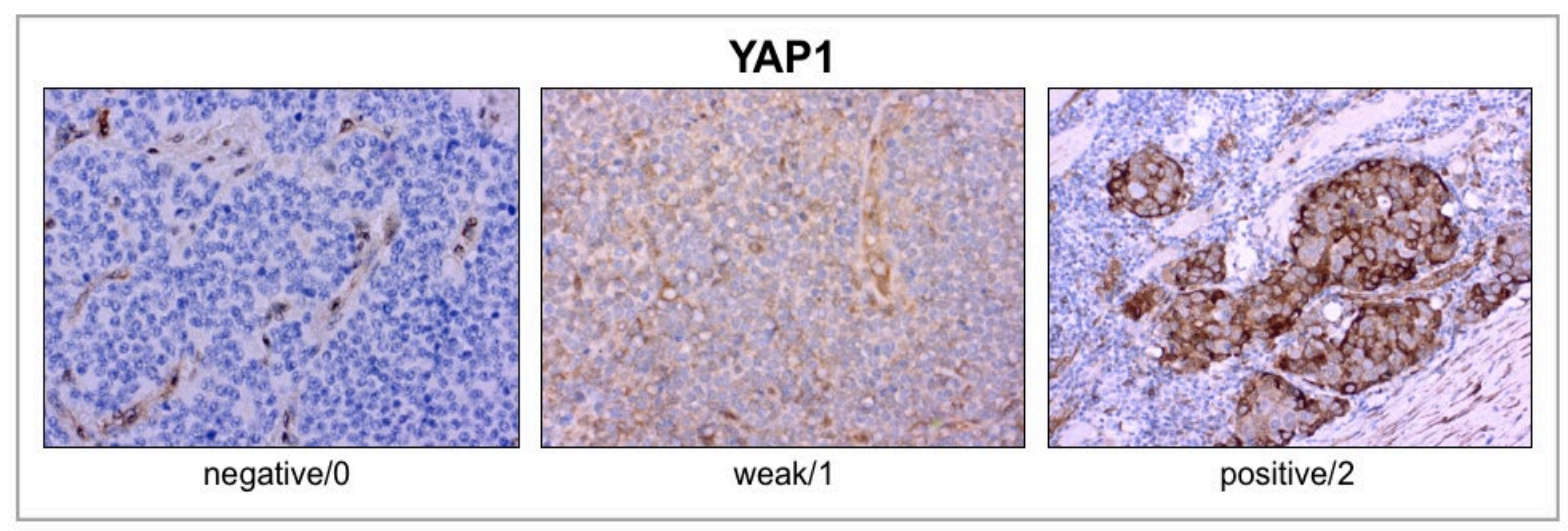

Figure 111: Immunohistochemistry staining intensity of YAP1. Left: YAP1 negative tumor cells, AC, objective x40. Middle: YAP1 weak tumor cells, AC, objective x40. Right: YAP1 positive tumor cells, LCNEC, objective x20.

Seven cases $(16 \%)$ showed positive immunolabeling and 37 cases $(84 \%)$ showed negative immunolabeling for YAP1 antibody. Positive immunolabeling was found in 1 of 10 TC (10\%), 3 of 23 AC (13\%), 2 of 8 LCNEC (25\%) and 1 of 3 SCC (33\%) (Fig. 112). The prevalence of positive YAP1 expression increased slightly with rising WHO tumor grade. 


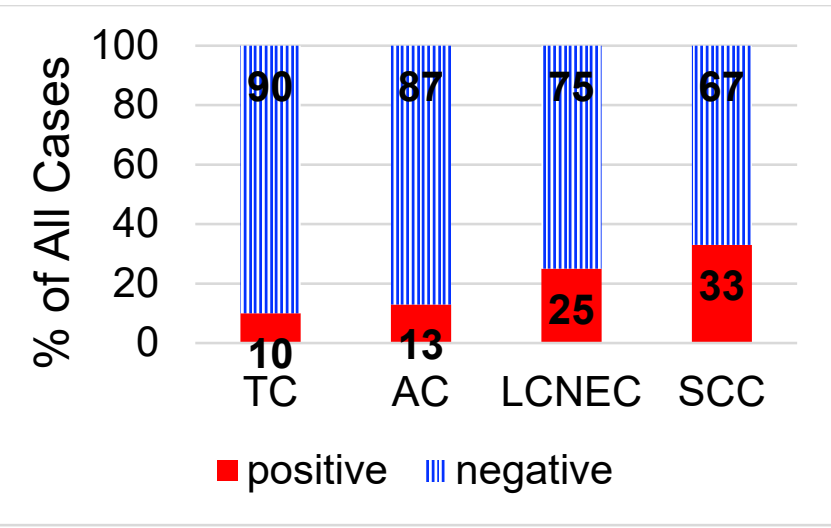

Figure 112: Distribution of YAP1 expression among WHO subgroups. The columns represent the percentage of cases with positive or negative antibody expression. Y-axis: percentage of stained cells, X-axis: WHO subgroup.

The Kaplan-Meier diagram divides YAP1 negative and positive tumors into two distinct survival groups $(p=0.00856)$ (Fig. 113). YAP1 negative tumors corresponded with longer overall survival. YAP1 positive tumors corresponded with dismal prognosis. The "positive" curve drops steeply within the first month.

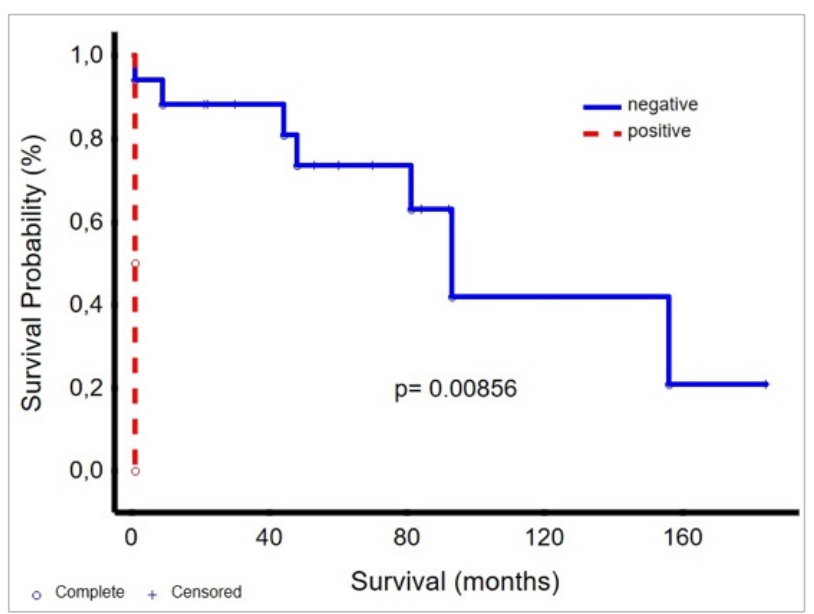

Figure 113: Survival analysis of TNET. The prognostic factor is two-tiered YAP1 expression. Y-axis: proportion surviving. $X$-axis: time in months.

YAP1 expression did not correlate with total chromosomal aberrations or CNI score (figure 114-115). Still, the boxplots show a tendency for YAP1 positive staining tumors to present with a wider and often higher range of chromosomal changes. 


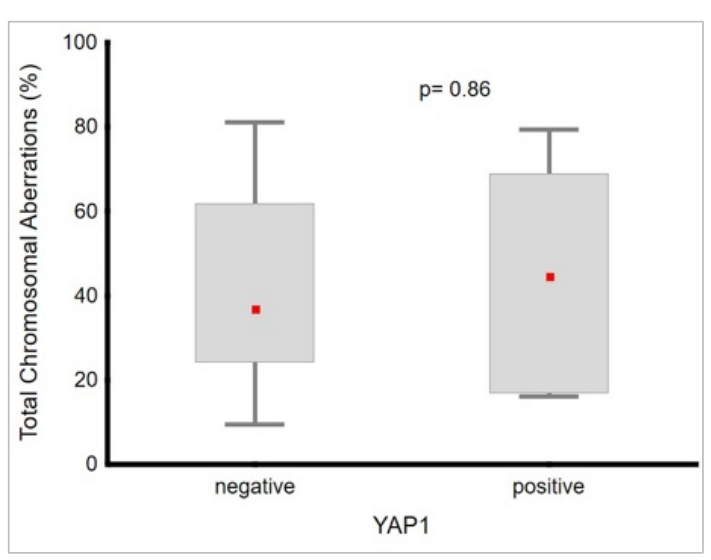

Figure 114: A correlation of YAP1 expression with total chromosomal aberrations. Y-axis: percentage of chromosomal aberrations. X-axis: negative or positive YAP1 staining.

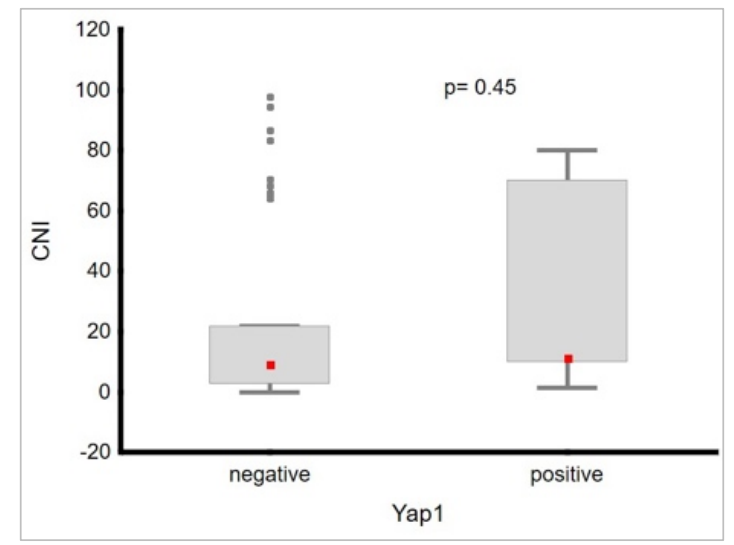

Figure 115: A correlation of YAP1 expression with CNI score. Y-axis: CNI score. X-axis: negative or positive YAP1 staining.

\subsubsection{TTF1 Immunohistochemical Analysis}

TTF1 was stained on 45 TNET (10 TC, 24 AC, 8 LCNEC, 3 SCC). The range of staining intensity is shown in figure 116. None of the TNET stained with an intensity of 2 for TTF1.

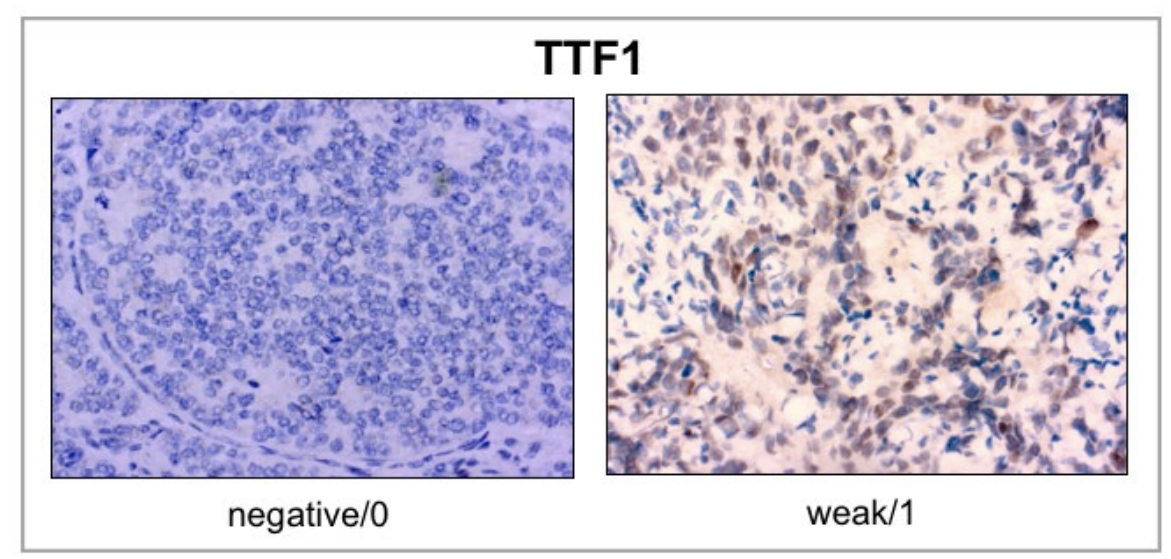

Figure 116: Immunohistochemistry staining intensity of TTF1. Left: TTF1 negative tumor cells, AC, objective x40. Right: TTF1 weak tumor cells, LCNEC, objective $x 40$.

All TNET were negative for TTF1 except for two LCNEC, which displayed a weak staining intensity of TTF1 (Fig. 117). 


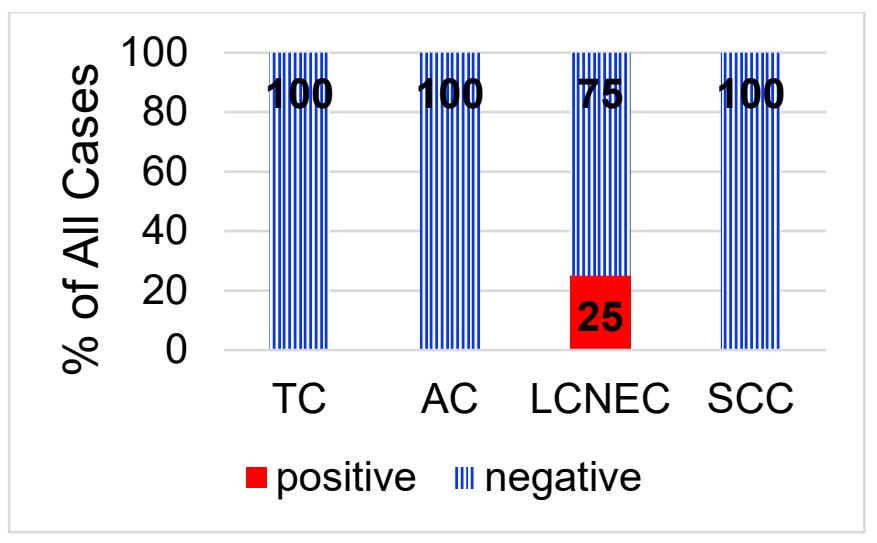

Figure 117: Distribution of TTF1 expression among WHO subgroups. The columns represent the percentage of cases with positive or negative antibody expression. Y-axis: percentage of stained cells, X-axis: WHO subgroup.

Due to the lack of positive staining cases, a survival analysis between groups was not possible. The same curve as in chapter 4.4 .4 results. When comparing TTF1 expression with chromosomal aberrations and CNI score, TTF1 positive tumors exhibited higher values in both parameters than TTF1 negative tumors. This was most likely due to the fact that there were only two positive cases.

\subsubsection{PAX8 Immunohistochemical Analysis}

PAX8 was stained in 45 TNET (10 TC, 24 AC, 8 LCNEC, 3 SCC). The spectrum of color intensity is illustrated in figure 118. The staining was nuclear.

In all, 21 tumors (47\%) showed positive PAX8 reactivity and 24 (53\%) showed no PAX8 reactivity. Specifically, 6 of 10 TC (60\%), 10 of 24 AC (42\%), 5 of 8 LCNEC $(63 \%)$ and 0 of 3 SCC (0\%) presented positive reactivity (Fig. 119).

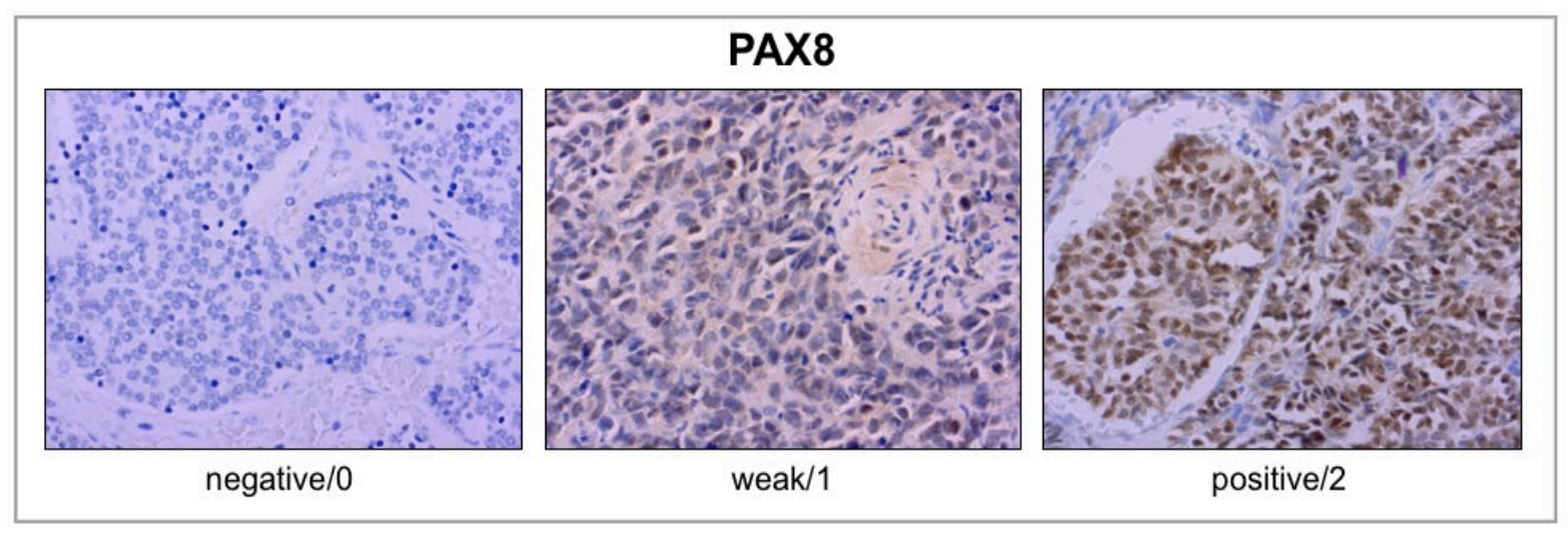

Figure 118: Immunohistochemistry staining intensity of PAX8. Left: PAX8 negative tumor cells, AC, objective x40. Middle: PAX8 weak negative tumor cells, LCNEC, objective x40. Right: PAX8 positive tumor cells, LCNEC, objective $x 40$. 


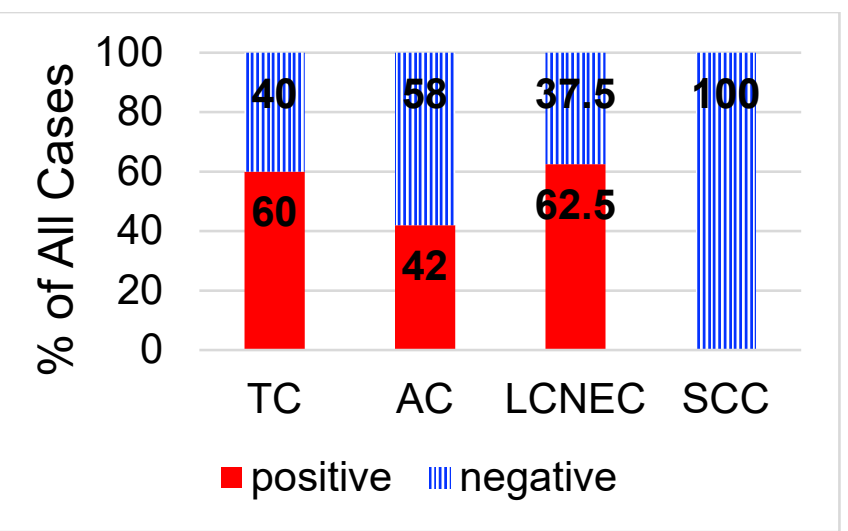

Figure 119: Distribution of PAX8 expression among WHO subgroups. The columns represent the percentage of cases with positive or negative antibody expression. Y-axis: percentage of stained cells, X-axis: WHO subgroup.

The Kaplan-Meier chart, figure 120, reveals that PAX8 expressing tumors associated with longer survival, while PAX8 negative tumors associated with shorter survival. This data was however not statistically significant $(p=0.25)$.

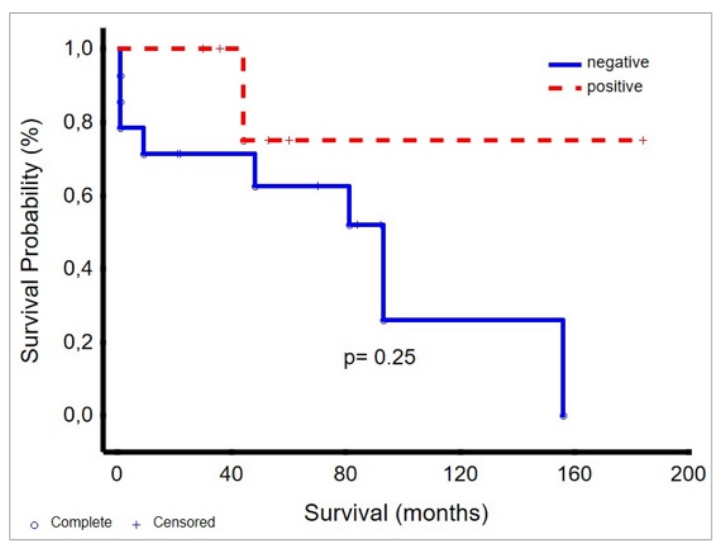

Figure 120: Survival analysis of TNET. The prognostic factor is two-tiered PAX8 expression. Y-axis: proportion surviving. $X$-axis: time in months.

PAX8 expression did not correlate significantly with chromosomal aberrations or $\mathrm{CNI}$ score (Fig.121-122). The averages of the two parameters had the same value for negative and positive staining tumors within each boxplot. 


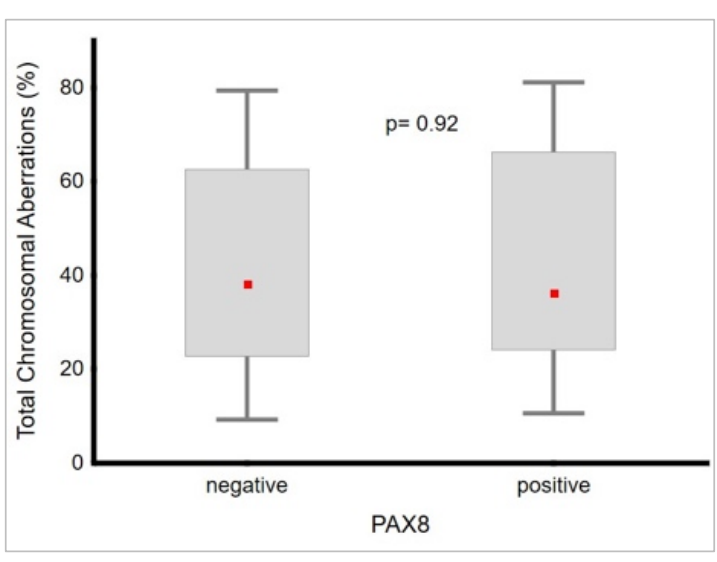

Figure 121: A correlation of PAX8 expression with total chromosomal aberrations. $Y$-axis: percentage of chromosomal aberrations. X-axis: negative or positive PAX8 staining.

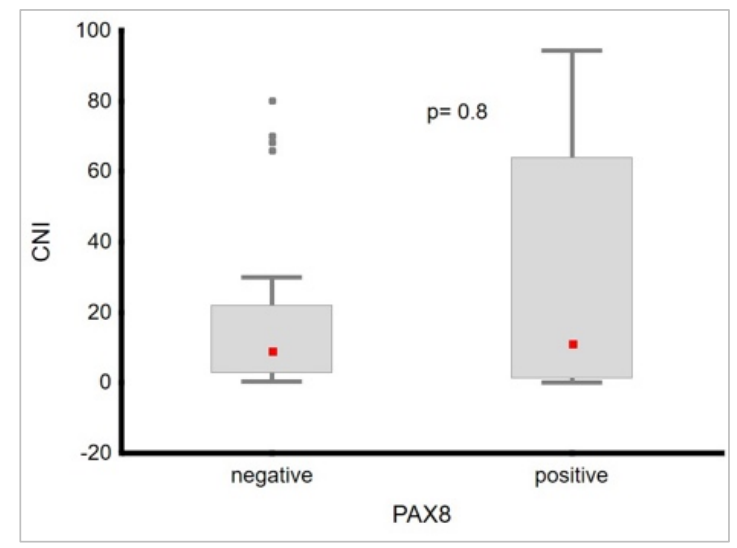

Figure 122: A correlation of PAX8 expression with CNI score. Y-axis: CNI score. X-axis: negative or positive PAX8 staining.

\subsubsection{Serotonin Immunohistochemical Analysis}

Serotonin antibody was processed on 45 TNET (10 TC, 24 AC, 8 LCNEC, 3 SCC). Serotonin has been found in NET of the small intestines (Schmitt et al. 2008; Niederle et al. 2016). However, in this TNET cohort, serotonin expression was not found. Figure 123 is an example of the negative serotonin expression. A Kaplan-Meier analysis and correlation with chromosomal changes was not possible.

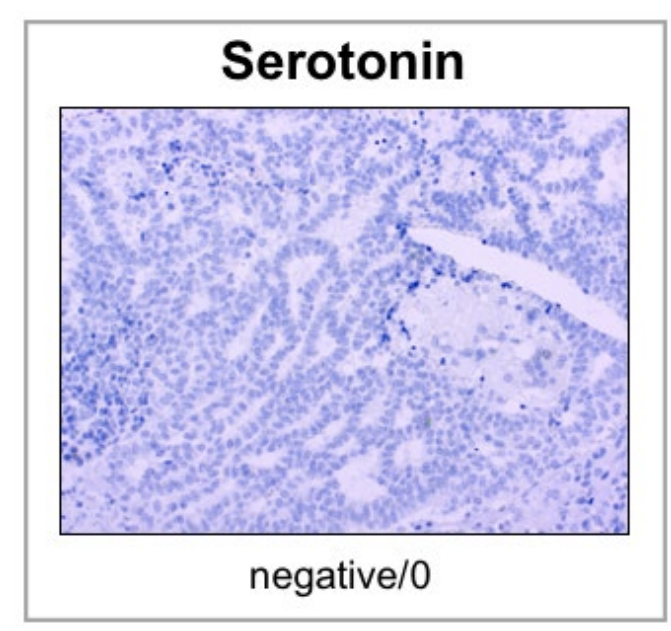

Figure 123: Immunohistochemistry staining intensity of Serotonin. Serotonin negative tumor cells, AC, objective $x 20$.

\subsubsection{CDX2 Immunohistochemical Analysis}

CDX2 antibody was stained on 45 TNET (10 TC, 24 AC, 8 LCNEC, 3 SCC). Figure 124 shows the color intensity spectrum of CDX2 antibody. Only negative/0 and intensity level 2 expression were amongst this cohort. The staining was nuclear. 


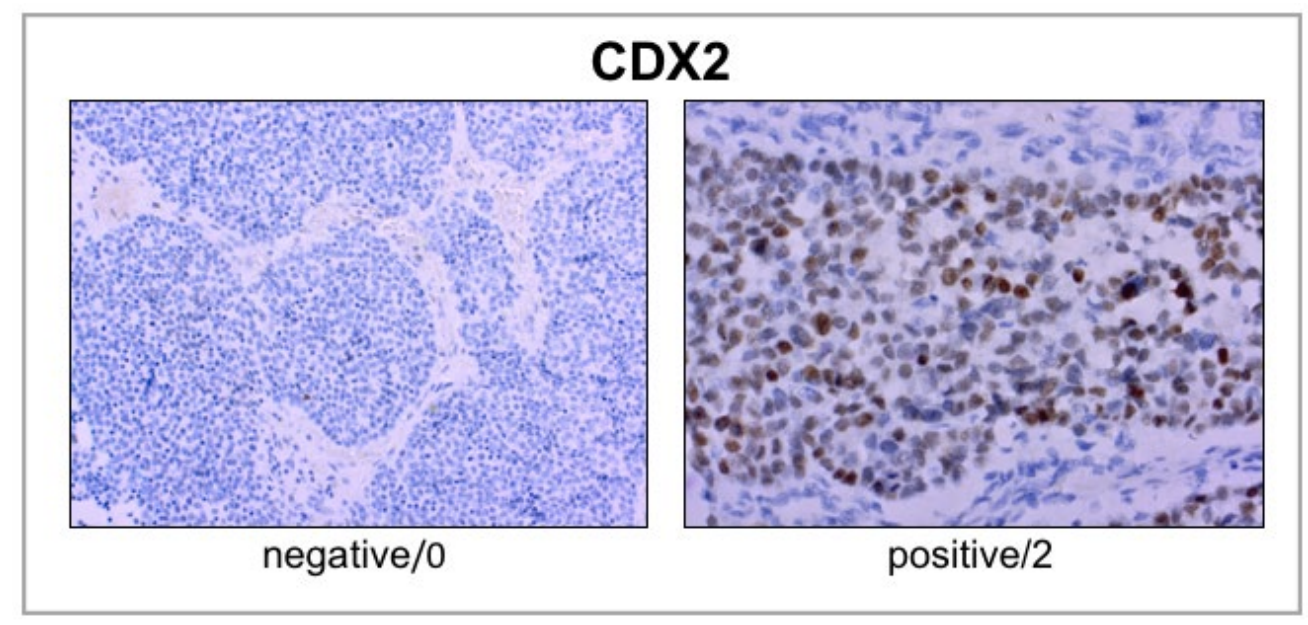

Figure 124: Immunohistochemistry staining intensity of CDX2. Left: CDX2 negative tumor cells, AC, objective x20. Right: CDX2 positive tumor cells, LCNEC, objective $x 40$.

In all, only one LCNEC showed immunoreactivity of CDX2. The other 44 TNET were negative. The survival time of LCNEC patient with CDX2 positive expression was only one month. Comparing chromosomal number variations with CDX2 was not possible because only one case was positive for CDX2.

\subsubsection{CD5 Immunohistochemical Analysis}

CD5 antibody shows immunoreactivity in thymic carcinoma (Jha et al. 2017), but has not been evaluated in thymic NET. CD5 expression was assessed in 45 TNET (10 TC, 24 AC, 8 LCNEC, 3 SCC). Figure 125 shows the spectrum of color intensity of the antibody. The antibody showed a cytoplasmic stain.

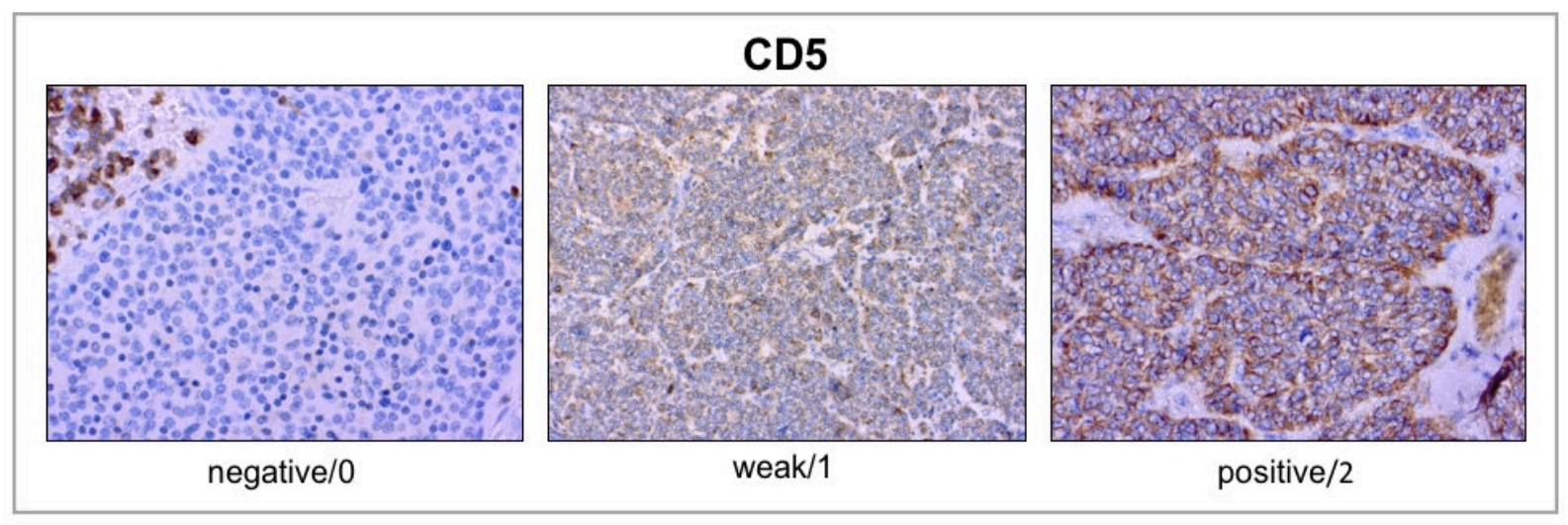

Figure 125: Immunohistochemistry staining intensity of CD5. Left: CD5 negative tumor cells, AC, objective x40. Middle: CD5 weak tumor cells, AC, objective x20. Right: CD5 positive tumor cells, AC, objective x40.

In all, six TNET (13\%) were positive and 39 (87\%) were negative for CD5 expression. 1 of 10 TC (10\%), 2 of 24 AC (8\%), 3 of 8 LCNEC (38\%) and 0 of 3 SCC $(0 \%)$ showed positive protein expression (Fig. 126). 


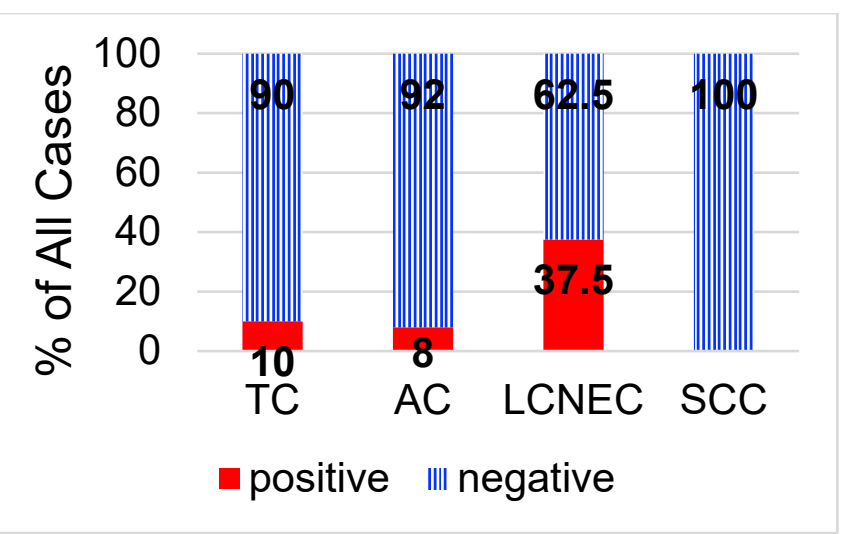

Figure 126: Distribution of CD5 expression among WHO subgroups. The columns represent the percentage of cases with positive or negative antibody expression. Y-axis: percentage of stained cells, X-axis: WHO subgroup.

A survival analysis was not possible due to lack of survival data within the positive group. When correlating CD5 expression to chromosomal copy number changes, there was no significant relationship. However, a trend between CNI score and CD5 expression was observed: CD5 positive tumors exhibited higher CNI on average, than CD5 negative tumors $(p=0.05)$. Total chromosomal aberrations and CD5 expression did not confirm this finding.

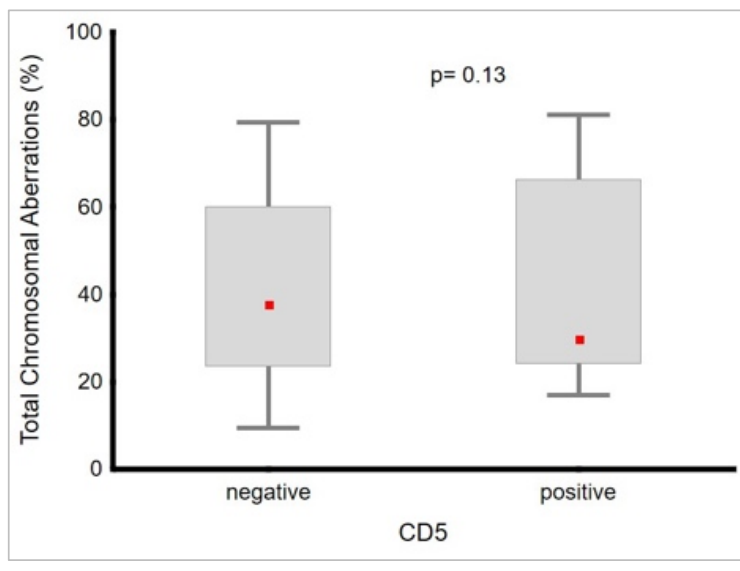

Figure 127: A correlation of CD5 expression with total chromosomal aberrations. Y-axis: percentage of chromosomal aberrations. X-axis: negative or positive CD5 staining.

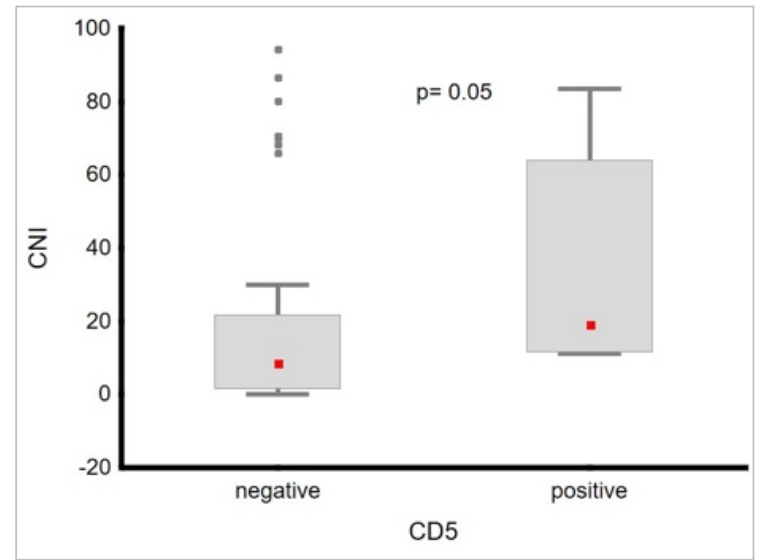

Figure 128: A correlation of CD5 expression with CNI score. Y-axis: CNI score. X-axis: negative or positive CD5 staining.

\subsubsection{CD117 Immunohistochemical Analysis}

45 TNET (10 TC, 24 AC, 8 LCNEC, 3 SCC) were assessed for CD117 expression. The spectrum of antibody color intensity is shown in figure 129. The antibody showed a cytoplasmic stain.

Altogether, six cases (13\%) showed positive CD117 expression and 39 (87\%) lacked CD117 expression. 1 of 10 TC (10\%), 3 of 24 AC (13\%), 2 of 8 LCNEC (25\%) and 0 
of 3 SCC (0\%) presented CD117 immunolabeling (Fig. 130). CD117 showed a similar expression pattern to CD5.

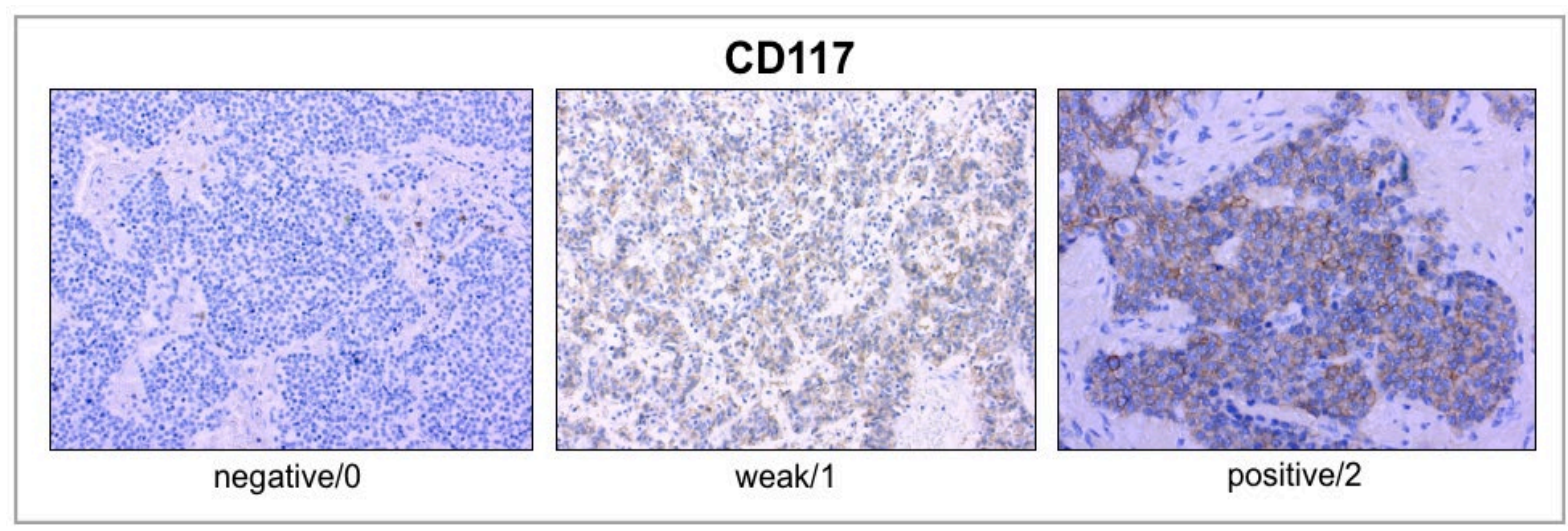

Figure 129: Immunohistochemistry staining intensity of CD117. Left: CD117 negative tumor cells, AC, objective x20. Middle: CD117 weak tumor cells, LCNEC, objective x20. Right: CD117 positive tumor cells, AC, objective x40.

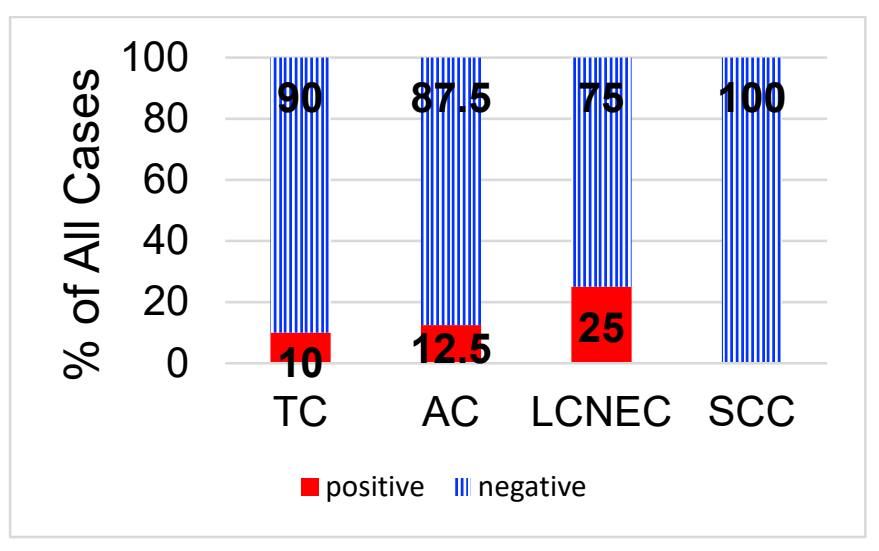

Figure 130: Distribution of CD117 expression among WHO subgroups. The columns represent the percentage of cases with positive or negative antibody expression. Y-axis: percentage of stained cells, X-axis: WHO subgroup.

In the Kaplan-Meier analysis, the tumors showing positive CD117 expression, demonstrated the best overall survival until 160 months, then survival probability dropped to zero (Fig. 131). CD117 negative tumors showed worse survival in the first 160 months after diagnosis, but survival probability remained constant after 90 months. The differentiation in survival between positive and negative tumors was not significant. 


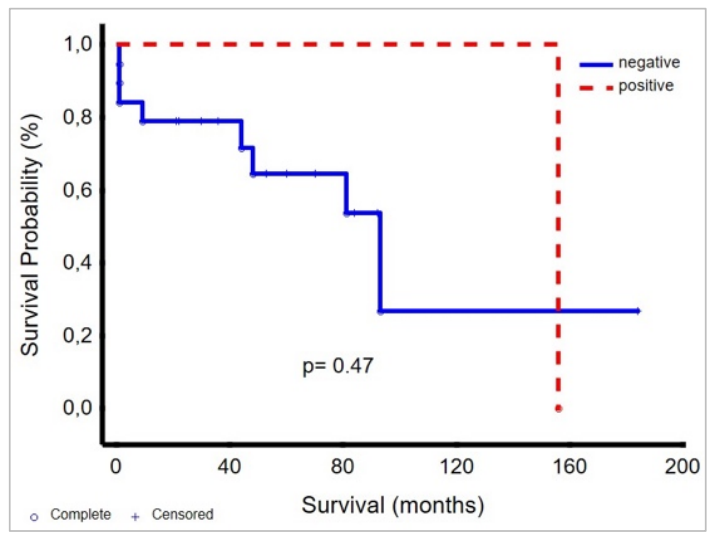

Figure 131: Survival analysis of TNET. The prognostic factor is two-tiered CD117 expression. Y-axis: proportion surviving. X-axis: time in months.

The boxplots in figure 132-133 illustrate that there is no significant separation of the number of chromosomal variants between positive and negative immunolabeling tumors in this cohort. Positive CD117 immunolabeling tumors tended to present with a wider range of chromosomal aberrations and $\mathrm{CNI}$ scores.

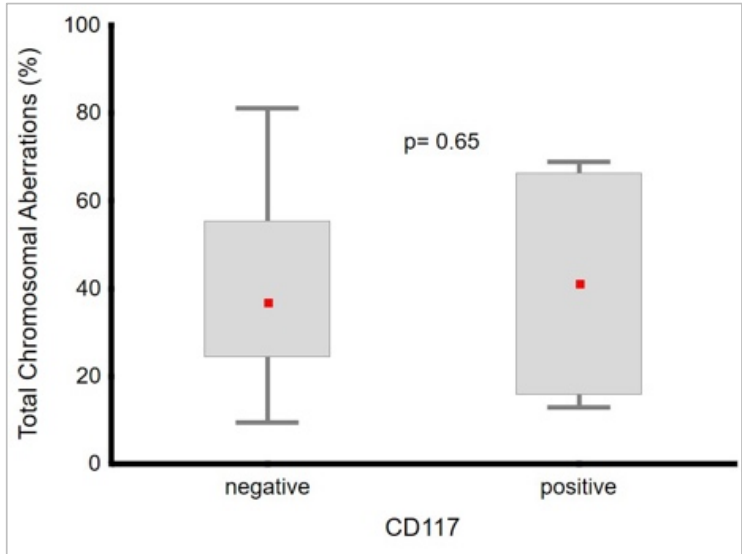

Figure 132: A correlation of CD117 expression with total chromosomal aberrations. Y-axis: percentage of chromosomal aberrations. X-axis: negative or positive CD117 staining.

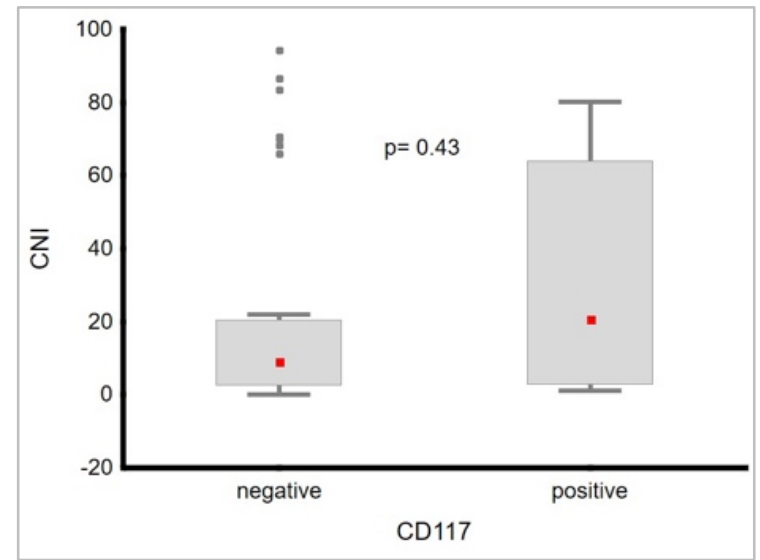

Figure 133: A correlation of CD117 expression with CNI score. Y-axis: CNI score. X-axis: negative or positive CD117 staining.

\subsection{Heatmap Cluster Analysis}

To identify subgroups that share specific characteristics, an unsupervised clustering analysis was carried out with the assistance of Dr. med Hanibal Bohnenberger (Institute of Pathology, University Medical Center Göttingen). All parameters that were analyzed in the investigations above, were incorporated into the analysis. This included: necrosis, the percent of total chromosomal aberrations, the percent of gains and percent of losses of chromosomes, CNI, ki67 labeling index, mitotic count, and all 21 immunohistochemical stain evaluations. For the analysis, each parameter evaluation was given a metric score. Cases were assigned 1 if necrosis was present 
and 0 if necrosis was absent in HE slides. IHC stains received points for the percentage of stained cells: $<25 \%=1$ point, $<50 \%=2$ points, $>50 \%=3$ points. These scores were then added to the intensity score: no staining $=0$ points, weak intensity= 1 point, strong intensity $=2$ points. The evaluation of percent of total chromosomal aberrations, gains, losses, CNI, ki67 and mitotic count were already metric variables. The scores of all parameters were then clustered. 40 TNET (8 TC, 21 AC, 8 LCNEC, 3 SCC) were integrated into the cluster analysis.

In the heatmap in figure 134, two areas with many red cells, representing higher values, come to attention. These are encircled in yellow. The in yellow encircled area on the left side of the heatmap (cluster 1) represents cases with higher scores of mitoses, ki67, EZH2, CNI, \% chromosomal aberrations, \% gains, \% losses, c-Myc, Yap1, p53, $\gamma \mathrm{H} 2 \mathrm{AX}$, keratin, necrosis, and p53. The TNET included in this cluster are one AC, five LCNEC, and three SCC. More high-grade tumors were associated with high values of these parameters. Interestingly, the five LCNEC within this cluster are LCNEC A, B, E, F, and $\mathrm{H}$ which were identified as NEC in Table 3. In the cluster on the right upper side of the heatmap (cluster 2), are cases which contained high levels of SDHA, chromogranin A, H3K27me3, RB1, DAXX, PAX 8, and ATRX expression. Some of these tumors also exhibited elevated levels of Calcitonin and CD5. The cases within this cluster are mostly TC and AC, and two LCNEC. The LCNEC are LCNEC D and G, which were classified as NET G3 in Table 3.

As mentioned in chapter 4.7.11, H3K27me3 showed almost inverse staining tendency to EZH2 in TC and SCC. H3K27me3 was found in almost all TC and was absent in SCC, while EZH2 was absent in TC and found in all SCC. The heatmap confirmed this correlation because EZH2 and H3K27me3 are found in opposite clusters. 


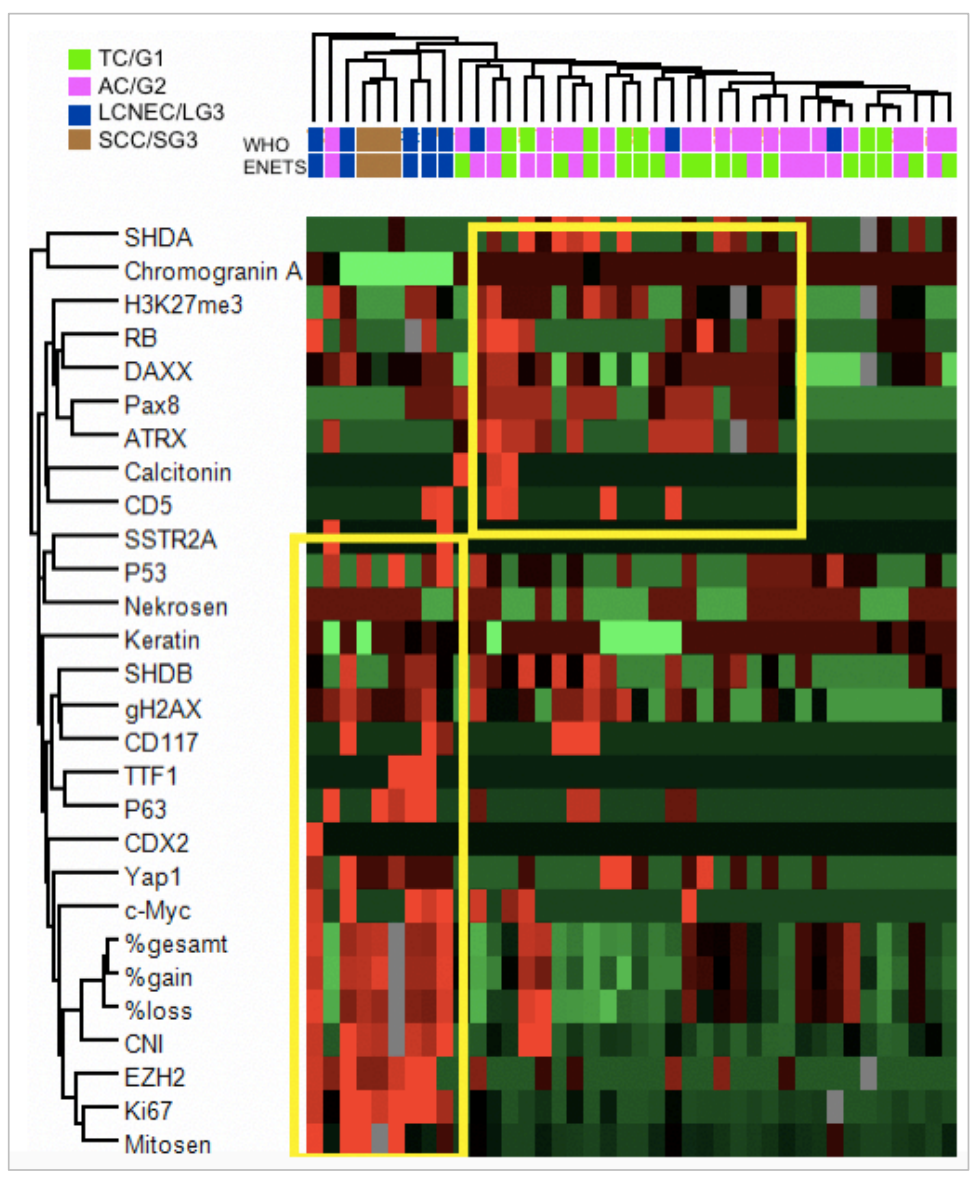

Figure 134: Unsupervised clustering analysis. The $Y$-axis portrays all markers evaluated in this study. The $X$-axis portrays the cases with WHO and ENETS classification. Two main clusters form.

The clusters were then tested for survival probability with a Kaplan-Meier analysis. The three groups consisted of cluster 1 and cluster 2 mentioned above, and cluster 3 which contains the remaining cases. The Kaplan-Meier diagram shows cluster 2 tumors to associate with the longest overall survival (Fig. 135). The cluster 2 curve is followed closely by the cluster 3 curve, however cluster 3 decreases more in survival probability at 50 months. Cluster 1 is associated with the shortest survival time. The prognosis is no longer than ten months.

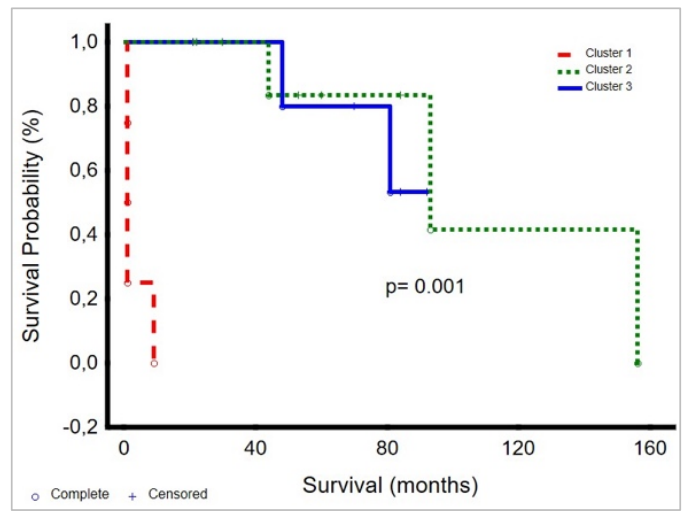

Figure 135: Cluster survival analysis. Y-axis: survival probability, X-axis: survival in months. Cluster 1 portrays the shortest survival time and cluster 2 portrays the longest survival. 


\subsection{Evidence of Morphologic and Genomic Progression in TNET}

A closer analysis of four cases that had more than one material available due to metastasis, tumor recurrence, or multiple sampling, was performed. In case \#1, the primary tumor was a LCNEC (16 mitoses per 10 HPF, ki67 n.a.), but its synchronous metastasis was classified as an AC (7 mitoses per 10 HPF, ki67 index 10). Interestingly, both tumors fell into the $\mathrm{CNl}_{\text {low }}$ cluster 1 and did not show major molecular differences. After further investigations, as mentioned in chapter 4.5.2, the LCNEC was later re-classified as a NET G3.

In case \#2, the primary tumor was an AC (4 mitoses per 10 HPF, ki67 index 3), while the metastasis, occurring three years after the primary tumor, was a LCNEC (16 mitoses per $10 \mathrm{HPF}$, ki67 index 17.5). This tumor was also later re-classified as a NET G3. The metastasis revealed some additional focal chromosomal gains and losses, but still, both the primary tumor and the metastasis fell into $\mathrm{CNl}_{\text {low }}$ cluster 1.

Case \#3 had two samples taken from the biopsy of the primary tumor (PT-1 and PT2) and one sample taken from the metastasis five years post PT-1 and PT-2. PT-1 was a TC (0 mitoses, ki67 index 1), PT-2 was an AC (10 mitoses per 10 HPF, ki67 index 12) and the metastasis was a LCNEC (16 mitoses per 10 HPF, ki67-index 16). The LCNEC was also later re-classified as a NET G3. PT-2 and the metastasis shared many chromosomal gains and losses that were not seen in PT1. Thus, PT1 fell into $\mathrm{CNI}_{\text {low }}$ cluster 1 , but PT2 and the metastasis fell into $\mathrm{CN}$ int cluster 2 . This shows the heterogeneity within single tumors and that intratumoral morphologic and genomic progression is possible.

Two samples were taken from the primary tumor in Case \#4 (PT-1 and PT-2). Both samples were classified as LCNEC (PT-1: 12 mitoses per 10 HPF, ki67 index 70, PT2: 8 mitoses per $10 \mathrm{HPF}$, ki67 index 70), and both fell into $\mathrm{CNl}$ int cluster 2. 


\section{Discussion}

Tumor grade is often utilized to place patients into prognostic groups, as it reflects the capability of tumor cells to grow and spread, ultimately affecting the clinical course (Edge 2010; Sobin et al. 2010; Pelosi et al. 2017a). Thus, grade has often been viewed as equivalent to a tumors' morphologic classification. However, how does one go about grading, when morphologic traits do not always reveal a tumor's true clinical behavior and underlying molecular backbone?

Since the introduction of the WHO pulmonary and thymic neuroendocrine tumor classification, the variables with the strongest prognostic significance have been a controversial discussion. Risk-stratification has been hindered by the absence of uniform grading classifications, by a lack of knowledge of the genetic background of NET and by recurring outliers within WHO subgroups. In this retrospective study, cytological proliferation indices, tumor morphology, immunohistochemistry and nextgeneration sequencing results were analyzed and incorporated to better define thymic neuroendocrine tumor subgroups. Proposed grading classifications from past retrospective studies were applied to the cohort of TNET, to evaluate coherence with prognosis and genetic alterations. We used molecular analyses (CNI) as the gold standard instead of the WHO classification to create a more prognosis-oriented threetiered grading system, and found a possible surrogate marker which mirrors TNET genomic instability.

\subsection{NET Classification Systems, Survival and Genomic Findings}

The tumor collective used in this study consisted of 107 thymic neuroendocrine tumors. More specifically, 22 were typical carcinoids, 51 were atypical carcinoids, 28 were large cell neuroendocrine carcinoma and six were small cell carcinoma. The basic classification used morphology, mitotic count and the presence of necrosis as defined in the WHO 2015 edition. Determining the ki67 proliferation index of these tumors showed that the ki67 spectrum of WHO subgroups manifests large overlap between subgroups. To determine how the ki67 index influences TNET grade, we applied the ENETS grading system to our cohort. The ENETS classification is a recognized grading system for gastro-entero-pancreatic NET. Additionally, the Pelosi et al. classification, a proposed classification system for pulmonary NET, was used on the TNET, to learn if thymic NET can again share a classification system with pulmonary NET, as is the case in the current WHO grading system. 
Applying the ENETS and Pelosi et al. NET grading systems to the TNET cohort led to a redistribution of $30 \%$ and $23 \%$ of cases, respectively. The ENETS system, which employs the ki67 labeling index as the grouping marker, and the Pelosi et al. NET grading system, which integrates ki67, mitotic count and necrosis, most often led to a downgrading of the WHO subgroups. The survival analyses of both the WHO and Pelosi et al. grading systems provided a per subgroup significant prognostic stratification in this collective. In contrast to our findings, in a study by Filosso et al., severe overlap was seen in survival analyses of TNET subgroups graded according to WHO (Filosso et al. 2015). TNET histology was unsuccessful in creating prognostic groups and survival times were similar in well-, intermediate- and poor-differentiated subtypes in their TNET cohort.

The ENETS classification, however, did not create four significant prognostic groups in our TNET cohort. The ki67 labeling index alone was not able to differentiate survival within the carcinoid and with the carcinoma subgroups. In other words, the survival times of typical and atypical carcinoids were very similar and not a graduated progression from better to worse survival. Furthermore, large cell and small cell carcinoma prognosis were indistinguishable. In a study by Dolcetta-Capuzzo et al., ENETS was the strongest predictor of survival in gastro-enteric NET, exposing four significant prognostic groups (Dolcetta-Capuzzo et al. 2013). Nonetheless, this was not the case for thymic NET.

Of these three grading systems, the WHO classification remained the most accurate in discerning prognosis because it created a significant survival curve for each of the four histologic subgroups. Nonetheless, it is interesting that the Pelosi et al. proposed grading system still provided significant subgroup stratification, even though it combined histologically different cases and consequently only created three subgroups. Despite the fact that ki67 was not able to stratify subgroups significantly, the ki67 proliferation index should be considered when determining the prognostic implication of TNET, as it can be a useful marker in uncovering tumor aggressiveness and separating borderline cases of AC versus LCNEC and NET G3 versus LCNEC (see below) (McCall et al. 2013).

Shallow whole genome sequencing of TNET confirmed an overall increase in chromosomal number alterations (CNA) and copy number instability score (CNI score) with an increase in WHO tumor grade, as was previously shown in a study by Ströbel et al. (Ströbel et al. 2014). It is remarkable that AC and LCNEC WHO subgroups 
displayed large areas of overlap in their spectrum of chromosomal aberrations. This was in part due to outliers in AC and LCNEC groups. One AC (CNI: 97.7) and even one TC (CNI: 94.4) displayed highly aberrant copy number variations more like those of high-grade NET, while some LCNEC contained minimal copy number variations more like those of carcinoids (CNI: 3.1). Interestingly, CNA in gene loci of tumor suppressor and driver genes, that were reported as common in pulmonary NET, were very rare in thymic $\mathrm{TC}$, more common in $\mathrm{AC}$ and showed a high frequency in LCNEC/SCC (Simbolo et al. 2017). Also, 11q deletions, which are a common finding in pulmonary TC and AC could not be confirmed in TNET (Walch et al. 1998).

A correlation of the ENETS and Pelosi et al. classifications with chromosomal number aberrations did not show a strong relationship. The ENETS system failed to separate CNA of the carcinoid group and of the carcinoma group. The Pelosi et al. NET classification was able to separate the number of chromosomal aberrations found in G3 from $G 2$ and $G 1$ groups but did not differentiate between $G 2$ and $G 1$. In all, these three grading systems, based on histologic characteristics, only moderately correlated with the genomic instability of TNET on a per case basis. Even direct correlations between mitotic count and chromosomal aberrations or between ki67 and chromosomal aberrations displayed only a weak interrelationship. On account of the molecular outliers within subgroups and the lack of a correlation between different grading systems with genetic aberrations, the question arises which tumor characteristics or markers best demonstrate genomic instability.

\subsection{CNI Cluster Analysis Reveals Three Major Molecular Clusters}

To discover the characteristics that reveal genomic instability, we first performed an unsupervised clustering analysis of the chromosomal copy number instability score data of 63 TNET cases. From this analysis of the CNI data, we identified three molecular clusters that we termed $\mathrm{CN}$ low, $\mathrm{CN}$ lintermediate and $\mathrm{CNI}$ high. Interestingly, all three molecular subgroups were heavily invaded by cases that would have been expected to fall into different risk groups and genetic clusters based on morphology and mitotic rate alone. In an attempt to reclassify our TNET cohort and encompass especially $A C$ and LCNEC outliers, cox proportional hazard regression was used to calculate $\mathrm{CNI}$ cutoffs between the $\mathrm{CNI}$ clusters. The significant cutoff for $\mathrm{CN}$ low was $<9$, for $\mathrm{CN}$ lintermediate 9-30, and for $\mathrm{CN} \mathrm{N}_{\text {high }}>30$ (Table 10). With these cutoff values, the 
three molecular subgroups provided significant prognostic relevance. The ki67 and mitotic count values overlapped extensively between the CNI clusters.

Table 10: Characteristics of CNI cluster groups

\begin{tabular}{|c|c|c|c|c|c|c|c|c|c|}
\hline CNI Cluster & $\begin{array}{l}\text { CNI } \\
\text { (median) }\end{array}$ & WHO & ENETS & $\begin{array}{l}\text { Pelosi } \\
\text { et al }\end{array}$ & $\begin{array}{l}\text { Ki67 } \\
\text { (median) }\end{array}$ & \begin{tabular}{|l|} 
Mitotic \\
Count \\
(median)
\end{tabular} & EZH2 & $\begin{array}{l}\text { Chromogranin } \\
\text { A }\end{array}$ & $\begin{array}{l}\text { Survival } \\
\text { (alive, dead, } \\
\text { no data) }\end{array}$ \\
\hline $\begin{array}{l}\mathrm{CNI}_{\text {low }} \\
(<9)\end{array}$ & $\begin{array}{l}0-8.8 \\
(2.4)\end{array}$ & $\begin{array}{l}6 \text { TC } \\
14 \text { AC } \\
2 \text { LCNEC (NET } \\
\text { G3) }\end{array}$ & $\begin{array}{l}11 \text { NET } \\
\text { G1 } \\
10 \text { NET } \\
\text { G2 } \\
1 \text { n.d. }\end{array}$ & $\begin{array}{l}10 \mathrm{G} 1 \\
11 \mathrm{G} 2 \\
1 \text { n.d. }\end{array}$ & $\begin{array}{l}1-19 \\
(2.6)\end{array}$ & $\begin{array}{l}0-27 \\
(3.5)\end{array}$ & $\begin{array}{l}17 \\
\text { negative } \\
5 \text { n.d. }\end{array}$ & $\begin{array}{l}19 \text { positive } \\
3 \text { n.d. }\end{array}$ & $\begin{array}{l}7 \mathrm{~A} \\
4 \mathrm{D} \\
11 \text { n.d. }\end{array}$ \\
\hline $\begin{array}{l}\text { CNI intermediate } \\
(9-30)\end{array}$ & $\begin{array}{l}9.05- \\
29.5 \\
(14.6)\end{array}$ & $\begin{array}{l}6 \text { TC } \\
10 \text { AC } \\
5 \text { LCNEC (3 } \\
\text { NET G3) }\end{array}$ & $\begin{array}{l}8 \text { NET G1 } \\
10 \text { NET } \\
\text { G2 } \\
1 \text { NEC } \\
2 \text { n.d. }\end{array}$ & $\begin{array}{l}7 \text { G1 } \\
10 \text { G2 } \\
4 \text { n.d. }\end{array}$ & $\begin{array}{l}1-66 \\
(4.6)\end{array}$ & $\begin{array}{l}0-25 \\
(5)\end{array}$ & $\begin{array}{l}15 \\
\text { negative } \\
6 \text { n.d. }\end{array}$ & $\begin{array}{l}15 \text { positive } \\
6 \text { n.d. }\end{array}$ & $\begin{array}{l}2 \mathrm{~A} \\
1 \mathrm{D} \\
16 \text { n.d. }\end{array}$ \\
\hline $\begin{array}{l}\text { CNI high } \\
(>30)\end{array}$ & $\begin{array}{l}30.04- \\
97.7 \\
(69.6)\end{array}$ & $\begin{array}{l}1 \mathrm{TC} \\
5 \mathrm{AC} \\
10 \mathrm{LCNEC} \\
4 \mathrm{SCC}\end{array}$ & $\begin{array}{l}5 \text { NET G2 } \\
9 \text { NEC } \\
2 \text { SCC } \\
4 \text { n.d. }\end{array}$ & $\begin{array}{l}11 \mathrm{G} 1 \\
5 \mathrm{G} 2 \\
4 \mathrm{G} 3 \\
10 \text { n.d. }\end{array}$ & $\begin{array}{l}3.5-90 \\
(55.5)\end{array}$ & $\begin{array}{l}0-100 \\
(12)\end{array}$ & $\begin{array}{l}4 \text { negative } \\
6 \text { positive } \\
10 \text { n.d. }\end{array}$ & $\begin{array}{l}6 \text { negative } \\
4 \text { positive } \\
10 \text { n.d. }\end{array}$ & $\begin{array}{l}1 \mathrm{~A} \\
3 \mathrm{D} \\
16 \text { n.d. }\end{array}$ \\
\hline
\end{tabular}

\subsubsection{EZH2 and Chromogranin A Associate with Genomic Instability}

The presence of TNET with "low grade morphology" within each CNI cluster clearly showed that ki67 and mitotic count cannot differentiate between these clusters, i.e. cannot predict genomic instability, due to large areas of overlap. However, EZH2 and Chromogranin A immunohistochemistry were able to separate low/intermediate from high $\mathrm{CNI}$ clusters. Cases in the $\mathrm{CNI}$ low and $\mathrm{CN}$ intermediate group were $100 \%$ positive for chromogranin $\mathrm{A}$ and $100 \%$ negative for $\mathrm{EZH} 2$, and cases in the $\mathrm{CN}$ high group were $60 \%$ positive for $\mathrm{EZH} 2$ and $60 \%$ negative for chromogranin $\mathrm{A}$. Within the $\mathrm{CN} \mathrm{I}_{\text {high }}$ group, all cases showed a reverse expression pattern between EZH2 and Chromogranin A, with the exception of one LCNEC, which displayed positive immunolabeling in both stains.

In fact, even cases not included in the clustering analysis shared the same expression pattern. TC and AC (i.e. expected to fall into the $\mathrm{CN}$ low/intermediate cluster) lacked $\mathrm{EZH} 2$, but strongly expressed chromogranin A. On the other hand, all SCC (i.e. CNIhigh) expressed EZH2 but lacked chromogranin A. LCNEC exhibited immunolabeling either similar to that of TC/AC or that of SCC. If molecular analyses are not available, EZH2 and Chromogranin A may be surrogate markers for TNET genomic instability. The overexpression of EZH2 was also a marker of poor prognosis. Similar discoveries have been made in pulmonary neuroendocrine tumors by Findeis-Hosey et al. 2011 and Bondgaard et al. 2012. In their studies, via immunohistochemistry, an enhanced expression of EZH2 was found in pulmonary LCNEC and SCC, but a decreased 
expression was found in carcinoids. However, EZH2 expression did not have a prognostic impact on pulmonary NET, as was the case in our thymic NET cohort. In Fernandez-Cuesta et al., EZH1 mutations and LOH were detected in pulmonary carcinoids (Fernandez-Cuesta et al. 2014). Whether EZH2 is one of the driver mutations in high-grade NET and a possible target for treatment approaches, remains to be elucidated (Bondgaard et al. 2012).

$\mathrm{EZH} 2$ or enhancer of zeste homolog 2 is the methyltransferase subunit of the polycomb repressive complex 2 . Through its methylation activity at histone $\mathrm{H} 3$, it trimethylates Lysin 27 (H3k27me3) (Gall Troselj et al. 2016). The methylation of histone $\mathrm{H} 3$ sets the chromatin in a repressive state in which PRC2's target genes (i.e. CDKN2A) are silenced (Margueron and Reinberg 2011). EZH1 is also part of PCR2. Gain of function mutations found in certain types of cancers result in an aberrant expression of EZH2. EZH2 overexpression keeps PRC2 target genes, including promotors of tumor suppressor genes, subdued. In this TNET cohort, no EZH2 mutations were found with targeted panel sequencing.

Considering that $\mathrm{EZH} 2$ activity produces $\mathrm{H} 3 \mathrm{k} 27 \mathrm{me} 3$, a positive correlation between these two is feasible. However, an inverted pattern of high levels of EZH2 and low levels of H3K27me3 was found in basal-like breast cancer cell lines and was linked with poorer prognosis (Bae et al. 2015; Gall Troselj et al. 2016). In our study, $66 \%$ of cases showed the same EZH2 and H3K27me3 staining, and 33\% showed opposite staining results. Also, almost all TC displayed positive immunolabeling of $\mathrm{H} 3 \mathrm{~K} 27 \mathrm{me} 3$ and lacked EZH2, and all SCC lacked H3K27me3 immunolabeling but expressed $\mathrm{EZH} 2$. This counter-intuitive observation is highly remarkable and its significance for TNET biology remains to be discovered.

In addition to calculating cutoff values for the $\mathrm{CNI}$ score, cutoff values were also determined for mitoses and ki67. It was noteworthy that both features showed strong significant cutoff values and hazard ratios at the upper end of the spectrum, at values above the threshold for LCNEC/SCC. This suggests that either another subgroup exists, as is the case in GEP NET, or that the current cutoff value must be raised (Konukiewitz et al. 2017). 


\subsection{WHO Large Cell Neuroendocrine Carcinoma Comprise a NET G3 Subgroup}

As mentioned above, especially LCNEC outliers presented with chromosomal number aberrations at the lower end of the spectrum, more comparable to the spectrum found in the carcinoid group, and thus often fell into the $\mathrm{CN} \mathrm{Nlow}_{\text {low }}$ and $\mathrm{CN}$ intermediate clusters, and not the expected $\mathrm{CN}_{\text {high }}$ cluster. Almost half of all LCNEC cases showed this phenomenon. On further inspection, these LCNEC also displayed well-differentiated neuroendocrine morphology and lower ki67 indices than the LCNEC that fell into the $\mathrm{CN}$ high cluster. Nevertheless, formal WHO criteria placed these cases into the highgrade/poorly differentiated category. Such cases have been documented before. In a study by Ahn et al., the author described three thymic NET that contained mitotic counts $>10$ per $10 \mathrm{HPF}$, formally categorizing these tumors as LCNEC (Ahn et al. 2012). Yet, the tumor morphology was so similar to the morphology of an $A C$, that the authors decided to classify the tumors as AC instead of LCNEC. Similar cases have also been described by Sobin et al. and Travis et al. in which pulmonary and gastroentero-pancreatic NET with an AC/G2 morphology were described, but the tumors exceeded the allowed mitotic count cutoff, thus requiring their classification as LCNEC (Sobin et al. 2010; Ströbel et al. 2014; Pelosi et al. 2017a).

We provisionally named such LCNEC falling into the $\mathrm{CN}$ low/intermediate categories, with carcinoid morphology and lower ki67 indices, "NET G3" in analogy to the terminology in GEP-NET (Basturk et al. 2015). In GEP-NET, a specific immunohistochemistry profile including ATRX, DAXX, p53, RB and SSTR2A is used to help differentiate NET G3 from NEC. On immunohistochemistry, thymic NET G3 expressed ATRX and variably DAXX, were negative for SSTR2A expression, showed positive/weak expression of RB and were negative for P53. On the other hand, the classic LCNEC, which gathered in the $\mathrm{CN}$ lhigh cluster and showed higher ki67 and poorer differentiation, were positive for ATRX and DAXX, negative for SSTR2A, partially negative for RB and partially positive for P53 (Konukiewitz et al. 2017). However, the most striking difference between NET G3 and LCNEC was that NET G3 exhibited positive expression of chromogranin $A$ and absent expression of $E Z H 2$, whereas LCNEC exhibited the reverse pattern, with absent expression of chromogranin $A$ and positive expression of EZH2. The combination of these two markers helped separate 
NET G3 from LCNEC and low/intermediate grade NET from NEC in general (see above).

C-Myc also exhibited an interesting expression pattern in TNET. All five LCNEC stained positive for c-Myc, while two NET G3 stained negative for c-Myc and one stained positive. In contrast to LCNEC, all SCC lacked c-Myc expression. Nevertheless, high genomic instability (high CNI score) correlated significantly with positive c-Myc expression. C-Myc amplification has been reported in the past as a hallmark of SCLC and has been reported in a borderline pulmonary atypical carcinoid (Peng et al. 2005; Swarts et al. 2011; Swarts et al. 2013). Further analyses are needed to determine the relationship between c-Myc expression and LCNEC.

\subsubsection{Targeted Sequencing Revealed Unexpected Mutations in LCNEC}

Sequencing of a limited gene panel in the 11 cases of LCNEC/NET G3 revealed one ATRX p.Q929E mutation in a NET G3 and revealed an unexpected high frequency of NF1 mutations in all NET G3 and LCNEC. NF1 mutations have also been described in pulmonary carcinoids and in pheochromocytomas, but at a much lower frequency (Asiedu et al. 2018). Neurofibromin, the protein encoded by the NF1 gene, is a negative regulator of the RAS/MAPK pathway. Thus, loss of NF1 can cause EGFR/BRAF drug resistance (Tetsu et al. 2016). The NF1 mutations and the additional KRAS and NRAS mutations found in two cases alludes to an important role of the RAS/MAPK signaling pathway in these tumors, meriting further investigation.

\subsection{Immunohistochemistry in Thymic NET}

To gain further insights into typical immunohistochemical staining profiles of TNET, an immunohistochemical analysis of 21 different stains was performed on our TNET collective. The most important immunohistochemical findings were already mentioned above, nevertheless some noteworthy observations were made. SSTR2A, Serotonin and CDX2 displayed negative immunoreactivity in all thymic NET cases (with two exceptions), unlike their gastro-entero-pancreatic counterpart, which often show strong expression of these markers (McCall et al. 2012; llett et al. 2015). These markers could be used to differentiate between GEP-NET metastases and thymic primary tumors. ATRX expression was negative in all SCC, but showed variable expression in TC, AC, and LCNEC. P53 expression had significant prognostic value in thymic NET, but did not directly correlate with TNET genomic instability, although a positive trend could be observed between positive p53 and higher genomic instability. 
It was also noteworthy that only tumors out of the carcinoid group (i.e. TC and AC) displayed positive SDHA expression, yet this was not the case for SDHB, which showed variable expression amongst WHO subgroups. $\gamma \mathrm{H} 2 \mathrm{AX}$ was prognostically significant in a three-tiered expression and showed significant correlation with total chromosomal aberrations. Two-tiered survival and a correlation with the CNI score did not reinforce the statistical significance. Keratin portrayed a constant expression pattern with rising WHO grade. On the other hand, YAP1 expression increased with rising WHO grade and its expression or lack thereof created significant survival groups. The remaining immunohistochemical markers (RB1, DAXX, CD5, CD117, PAX8, Calcitonin) showed variable expression amongst WHO subgroups and did not show significant correlation with survival or the genomic instability of thymic NET.

\subsection{Morphologic and Genomic Progression in TNET}

A detailed study of the few TNET cases with both primary tumors and metastases available, provided further interesting insights. Cases with metachronous metastases showed evidence of morphological and genomic progression. For example, in one case the primary tumor was an AC, but the metastasis progressed to a NET G3. This was also accompanied by an increase of mitotic rate and/or ki67 index. The primary tumor and metastasis also progressed from one CNI cluster to another, confirming previous impressions that NET can at least evolve from low to intermediate grade tumors by gaining further genomic alterations (Fabbri et al. 2017). These findings support the view that low and intermediate grade TNET represent a continuum rather than independent subtypes. To establish if low/intermediate grade tumors can further progress to high-grade tumors, additional research will be required (Pelosi et al. 2018).

Furthermore, one case showed grade heterogeneity within the primary tumor. Two samples were taken from this tumor at the same time. The first sample was diagnosed as a TC with zero mitoses and the second sample was diagnosed as an AC with ten mitoses. This further emphasizes that low-/intermediate-grade tumors represent a spectrum rather than distinct entities.

If low-grade tumors can progress to intermediate-grade tumors, further investigations will be needed to determine if low-/intermediate-grade thymic NET can also contain concurrent high-grade components. In the past, grade heterogeneity has been described in NET of the gastro-entero-pancreatic system (Tang et al. 2016b). In these organs, well-differentiated NET can achieve proliferative rates in the high-grade 
(LCNEC/SCC) range dispersed throughout the tumor (Yang et al. 2011; Tang et al. 2016b). Such discrepancies in tumor diagnosis complicate therapeutic and prognostic stratification.

\subsection{Proposal for an Integrated "Morpho-Molecular" TNET Classification}

Altogether, our data support a clinical and genomic three-tiered system to better predict tumor behavior. Our results revealed that tumor morphology alone is not reliable enough to classify TNET for precise risk stratification of individual patients. In order to incorporate our findings of the morphological and molecular features of TNET, we propose a tentative "morpho-molecular" classification which includes our prognostically relevant, calculated cutoff values for CNI score, ki67, and mitotic count, among others. Similar to the Pelosi et al. classification proposal, the three groups are named Thy-NET G1-G3 (Pelosi et al. 2017a). The cutoff values between Thy-NET G1 and $\mathrm{G} 2$ are $<10$ vs. $>10$ mitoses (HR 0.38, p = 0.03), <9\% vs. $>9 \%$ ki67 index (HR $0.07, p=0.016$ ), and $<9$ vs. $>9 \mathrm{CNI}$ score (HR 0.10, $p=0.047$ ). The cutoff values separating Thy-NET G2 from G3 are <30 vs. $>30$ mitoses (HR 0.02, p = 0.00023), $<48 \%$ vs. $>48 \%$ ki67 index (HR 0.15, p = 0.033), and <30 vs. $>30 \mathrm{CNI}$ score (HR 0.11 , $p=0.018)$. Chromogranin $A$ and $E Z H 2$ immunohistochemistry are utilized as markers for molecular instability, differentiating Thy-NET G1/G2 from Thy-NET G3. The criteria for each of the three groups are summarized in Table 11. 
Table 11: Integrated morpho-molecular TNET classification. The three-tiered classification is based on the calculated cutoff values for mitotic count, ki67, and CNI score. EZH2 and Chromogranin A immunohistochemistry are substitute markers to differentiate between low/intermediate and high genomic instability.

\begin{tabular}{|l|l|l|l|l|l|}
\hline $\begin{array}{c}\text { Morpho- } \\
\text { molecular } \\
\text { group }\end{array}$ & $\begin{array}{c}\text { Morphology } \\
\text { WHO subtype }\end{array}$ & $\begin{array}{c}\text { Mitotic count } \\
\text { (per } \mathbf{2} \mathbf{~ m m}^{2} \text { or } \\
10 \mathrm{HPF})\end{array}$ & ki67-index & CNI score & IHC \\
\hline Thy-NET G1 & $\begin{array}{l}\text { carcinoid } \\
\left(\mathrm{TC}, \mathrm{AC},{ }^{\text {"NET }}\right. \\
\text { G3") }\end{array}$ & $<10$ & $<9$ & $<9$ & $\begin{array}{c}\text { CGA pos } \\
\text { EZH2 neg }\end{array}$ \\
\hline Thy-NET G2 & $\begin{array}{l}\text { carcinoid or } \\
\text { carcinoma } \\
(\text { TC, AC, "NET } \\
\text { G3", LCNEC) }\end{array}$ & $10-29$ & $9-47$ & $9-29$ & $\begin{array}{l}\text { CGA pos } \\
\text { EZH2 neg }\end{array}$ \\
\hline Thy-NET G3 & $\begin{array}{l}\text { carcinoid or } \\
\text { carcinoma } \\
\text { (LCNEC, SCC) }\end{array}$ & $\geq 30$ & $\geq 48$ & $\geq 30$ & $\begin{array}{l}\text { CGA +/- } \\
\text { EZH2 pos }\end{array}$ \\
\hline
\end{tabular}

It is important to note, that because of the leading role the CNI score plays in this classification, using histology and immunohistochemistry alone could lead to misclassification of some TNET. In this collective, by excluding the CNI score, 8/34 (23.5\%) of Thy-NET G1 would be misclassified as Thy-NET G2 and 10/13 (77\%) of Thy-NET G2 would be misclassified as Thy-NET G1. Further, although high-grade morphology, high proliferation indices, negative chromogranin and positive EZH2 expression were strong indicators for Thy-NET G3 (or LCNEC/SCC), the absence of these markers did not exclude high genomic instability. In our collective, all three carcinoids with high CNI (in cluster 3 in Fig. 40) displayed proliferation at the lower end of the spectrum and were negative for $\mathrm{EZH} 2$ and positive for chromogranin. These cases would have been misclassified as either Thy-NET G2 or G1 by using morphology and immunohistochemistry alone.

From these results, we hypothesize that the most pertinent distinction will be the differentiation of Thy-NET G1/G2 from Thy-NET G3. In other words, tumors with $\geq 30$ mitoses per $2 \mathrm{~mm}^{2}$ or a ki67 index $\geq 48$, positive expression of $E Z H 2$ and loss of chromogranin A will have to be treated differently from tumors falling below those thresholds. This is similar to gastro-entero-pancreatic NET, in which a ki67 of $\geq 55 \%$ conveys a clinically important cutoff for the decision to use platinum-based chemotherapy (Sorbye et al. 2013). The therapeutic consequences this new threshold signifies in TNET is yet to be uncovered. 


\section{Summary}

The goal of this study was to deepen the understanding of important prognostic markers and the molecular background of thymic neuroendocrine tumors, ultimately allowing an allocation of these features to TNET subgroups. In order to achieve this, this study included determining the histological features, expression of various markers on immunohistochemistry, and chromosomal alterations within TNET.

Altogether, this study revealed strong evidence that TNET fall into three major molecular categories. In addition, we established the novel entity of NET G3, which had not been recognized in the thymus before. We clearly showed that morphology alone is not sufficient in recreating and reflecting these categories. For example, the WHO, ENETS, and Pelosi et al. classifications, all relying on tumor morphology/histology, could not reflect the molecular clusters that formed in unsupervised clustering analyses on a per-case basis. This investigation showed that typical and atypical carcinoids, as well as the newly defined NET G3, fall into low- and intermediate-grade molecular categories. Within these two molecular groups, morphological and molecular progression is possible. Further, LCNEC fall into either intermediate- or high-grade molecular categories, while SCC remain in the high-grade molecular category. To uncover if progression from differentiated NET (i.e. TC/AC/NET G3) to undifferentiated NEC (i.e. LCNEC/SCC) is possible, further studies will be required.

Altogether, these results support that a three-tiered morpho-molecular grading system better reflects the biology of TNET than the traditional four-tiered histological WHO classification. EZH2 and chromogranin A immunohistochemistry could help to differentiate between tumors of the low-/intermediate- and the high-grade category of TNET. Lastly, this study acknowledged that TNET share many features with their pulmonary and gastrointestinal and pancreatic counterpart, supporting the adjustment of general criteria defined for these organs. However, the data in this study also revealed organ-specific features, exclusive to thymic NET, thus far. It is important for future investigations to deepen the insight into the shared and unique characteristics of TNET and to take advantage of them for treatment purposes and patient risk stratification. 


\section{References}

Ahn S, Lee JJ, Ha SY, Sung CO, Kim J, Han J (2012): Clinicopathological Analysis of 21 Thymic Neuroendocrine Tumors. Korean J Pathol $\underline{46}$, 221-225

Asiedu M, Thomas C, Dong J, Schulte S, Khadka P, Sun Z, Kosari F, Jen J, Molina J, Vasmatzis G, et al. (2018): Pathways Impacted by Genomic Alterations in Pulmonary Carcinoid Tumors. Clin Cancer Res $\underline{24}$, 1691-1704

Bae WK, Yoo KH, Lee JS, Kim Y, Chung IJ, Park MH, Yoon JH, Furth P, Hennighausen $L$ (2015): The methyltransferase $E Z H 2$ is not required for mammary cancer development, although high EZH2 and low H3K27me3 correlate with poor prognosis of ER-positive breast cancers. Mol Carcinog $\underline{54}$, $1172-1180$

Basturk O, Yang Z, Tang L, Hruban R, Adsay V, McCall C, Krasinskas A, Jang KT, Frankel WL, Balci S, et al. (2015): The high-grade (WHO G3) pancreatic neuroendocrine tumor category is morphologically and biologically heterogenous and includes both well differentiated and poorly differentiated neoplasms. Am J Surg Pathol $\underline{39}$, 683-690

Bohnenberger H, Dinter H, König A, Ströbel P (2017): Neuroendocrine tumors of the thymus and mediastinum. J Thorac Dis $\underline{9}$, S1448-S1457

Bondgaard AL, Poulsen TT, Poulsen HS, Skov BG (2012): Different expression of $\mathrm{EZH} 2, \mathrm{BMI} 1$ and Ki67 in low and high grade neuroendocrine tumors of the lung. Cancer Biomark 11, 123-128

Booth D, Takagi M, Sanchez-Pulido L, Petfalski E, Vargiu G, Samejima K, Imamoto N, Ponting C, Tollervey D, Earnshaw W, et al. (2014): Ki-67 is a PP1-interacting protein that organises the mitotic chromosome periphery. eLife $\underline{3}$, e01641

Brcic L, Heidinger M, Popper H (2016): Neuroendokrine Neoplasien des Mediastinums. Pathologe $\underline{37}, 434-440$

Chetty R, Batitang S, Govender D (1997): Large cell neuroendocrine carcinoma of the thymus. Histopathology $\underline{31}, 274-276$

de Montpreville V, Macchiarini P, Dulmet E (1996): Thymic neuroendocrine carcinoma (carcinoid): a clinicopathologic study of fourteen cases. J Thorac Cardiovasc Surg $\underline{111}, 134-141$

Dolcetta-Capuzzo A, Villa V, Albarello L, Franchi G, Gemma M, Scavini M, Palo S, Orsenigo E, Bosi E, Doglioni C, et al. (2013): Gastroenteric neuroendocrine neoplasms classification: Comparison of prognostic models. Cancer $\underline{119}, 36-$ 44

Duraiyan J, Govindarajan R, Kaliyappan K, Palanisamy M (2012): Applications of immunohistochemistry. J Pharm Bioallied Sci 4, S307-S309 
Edge S, Byrd D, Compton C, Fritz A, Greene F, Trottie A: AJCC Cancer Staging Manual. 7th Edition; Springer-Verlag, New York 2010

Fabbri A, Cossa M, Sonzogni A, Bidoli P, Canova S, Cortinovis D, Abbate MI, Calabrese F, Nannini N, Lunardi F, et al. (2017): Thymus neuroendocrine tumors with CTNNB1 gene mutations, disarrayed ß-catenin expression, and dual intra-tumor Ki-67 labeling index compartmentalization challenge the concept of secondary high-grade neuroendocrine tumor: a paradigm shift. Virchows Arch 471, 31-47

Fernandez-Cuesta L, Peifer M, Lu X, Sun R, Ozretić L, Seidal D, Zander T, Leenders F, George J, Müller C, et al. (2014): Frequent mutations in chromatinremodeling genes in pulmonary carcinoids. Nat Commun $\underline{5}, 3518$

Ferolla P, Falchetti A, Filosso P, Tomassetti P, Tamburrano G, Avenia N, Daddi G, Puma F, Ribacchi R, Santeusanio F, et al. (2005): Thymic Neuroendocrine Carcinoma (Carcinoid) in Multiple Endocrine Neoplasia Type 1 Syndrome: The Italian Series. J Clin Endocrinol Metab 90, 2603-2609

Filosso PL, Yao X, Ahmad U, Zhan Y, Huang J, Ruffini E, Travis W, Lucchi M, Rimner A, Antonicelli A, et al. (2015): Outcome of primary neuroendocrine tumors of the thymus: A joint analysis of the International Thymic Malignancy Interest Group and the European Society of Thoracic Surgeons databases. J Thorac Cardiovasc Surg 149, 103-109.e2

Findeis-Hosey J, Huang J, Li F, Yang Q, McMahon L, Xu H (2011): High-grade neuroendocrine carcinomas of the lung highly express enhancer of zeste homolog 2, but carcinoids do not. Hum Pathol $\underline{42}$, 867-872

Frilling A, Akerström G, Falconi M, Pavel M, Ramos J, Kidd M, Modlin I (2012): Neuroendocrine tumor disease: an evolving landscape. Endocr Relat Cancer $\underline{19}, \mathrm{R} 163-185$

Futreal PA, Coin L, Marshall M, Down T, Hubbard T, Wooster R, Rahman N, Stratton M (2004): A Census of Human Cancer Genes. Nat Rev Cancer $\underline{4}, 177-183$

Gal A, Kornstein M, Cohen C, Duarte I, Miller J, Mansour K (2001): Neuroendocrine tumors of the thymus: a clinicopathological and prognostic study. Ann Thorac Surg $\underline{72}, 1179-1182$

Gall Troselj K, Novak Kujundzic R, Ugarkovic D (2016): Polycomb repressive complex's evolutionary conserved function: the role of EZH2 status and cellular background. Clin Epigenetics $\underline{8}, 55$

Gaur P, Leary C, Yao J (2010): Thymic neuroendocrine tumors: a SEER database analysis of 160 patients. Ann Surg 251, 1117-1121

Goto K, Kodama T, Matsuno Y, Yokose T, Asamura H, Kamiya N, Shimosato Y (2001): Clinicopathologic and DNA cytometric analysis of carcinoid tumors of the thymus. Mod Pathol 14, 985-994 
Hochwald S, Zee S, Conlon K, Colleoni R, Louie O, Brennan M, Klimstra D (2002): Prognostic factors in pancreatic endocrine neoplasms: an analysis of 136 cases with a proposal for low-grade and intermediate-grade groups. J Clin Oncol $\underline{20}$, 2633-2642

llett EE, Langer S, Olsen IH, Federspiel B, Kjær A, Knigge U (2015): Neuroendocrine Carcinomas of the Gastroenteropancreatic System: A Comprehensive Review. Diagnostics (Basel) $\underline{5}, 119-176$

Jha V, Sharma P, Mandal AK (2017): Utility of cluster of differentiation 5 and cluster of differentiation 117 immunoprofile in distinguishing thymic carcinoma from pulmonary squamous cell carcinoma: A study on 1800 nonsmall cell lung cancer cases. Indian J Med Paediatr Oncol $\underline{38}$, 430-433

Jiao Y, Shi C, Edil B, de Wilde R, Klimstra D, Maitra A, Schulick R, Tang L, Wolfgang C, Choti M, et al. (2011): DAXX/ATRX, MEN1, and mTOR pathway genes are frequently altered in pancreatic neuroendocrine tumors. Science $\underline{331}, 1199$ 1203

Kaltsas G, Besser GM, Grossman A (2004): The diagnosis and medical management of advanced neuroendocrine tumors. Endocr Rev 25, 458-511

Khan M, Luong T, Watkins J, Toumpanakis C, Caplin M, Meyer T (2013): A comparison of $\mathrm{Ki}-67$ and mitotic count as prognostic markers for metastatic pancreatic and midgut neuroendocrine neoplasms. Br J Cancer $\underline{108}, 1838-$ 1845

Kim JY, Hong S-M (2016): Recent Updates on Neuroendocrine Tumors From the Gastrointestinal and Pancreatobiliary Tracts. Arch Pathol Lab Med 140, 437448

Klimstra D, Modlin I, Coppola D, Lloyd R, Suster S (2010): The pathologic classification of neuroendocrine tumors: a review of nomenclature, grading, and staging systems. Pancreas $\underline{39}, 707-712$

Klimstra D, Beltran H, Lilenbaum R, Bergsland $E$ : The spectrum of neuroendocrine tumors: histologic classification, unique features and areas of overlap. In: Dizon D, Pennell N, Pickell L, Carter D, Adams H, Dottellis D (Eds.): American Society of Clinical Oncology 2015 Educational Book. 35th Edition; American Society of Clinical Oncology, Alexandria 2015, 92-103

Klöppel G (2017): Neuroendocrine Neoplasms: Dichotomy, Origin and Classifications. Visc Med 33, 324-330

Konukiewitz B, Schlitter AM, Jesinghaus M, Pfister D, Steiger K, Segler A, Agaimy A, Sipos B, Zamboni G, Weichert W, et al. (2017): Somatostatin receptor expression related to TP53 and RB1 alterations in pancreatic and extrapancreatic neuroendocrine neoplasms with a Ki67-index above 20\%. Mod Pathol $\underline{30}$, 587-598 
Kulke M, Lenz H-J, Meropol N, Posey J, Ryan D, Picus J, Bergsland E, Stuart K, Tye L, Huang X, et al. (2008): Activity of sunitinib in patients with advanced neuroendocrine tumors. J Clin Oncol 트, 3403-3410

Li H, Durbin R (2009): Fast and accurate short read alignment with Burrows-Wheeler transform. Bioinformatics $\underline{25}, 1754-1760$

Ma K, Liu Y, Xue Z, Chu X (2017): Treatment, prognostic markers, and survival in thymic neuroendocrine tumors. Medicine (Baltimore) $\underline{96}$, e7842

Margueron R, Reinberg D (2011): The Polycomb complex PRC2 and its mark in life. Nature $\underline{469}, 343-349$

Marinoni I, Kurrer A, Vassella E, Dettmer M, Rudolph T, Banz V, Hunger F, Pasquinelli S, Speel E-J, Perren A (2014): Loss of DAXX and ATRX are associated with chromosome instability and reduced survival of patients with pancreatic neuroendocrine tumors. Gastroenterology 146, 453-460.e5

Marx A, Shimosato $\mathrm{Y}$, Kuo T, Chan J, Travis W, Wick M: Thymic neuroendocrine tumours. In: Travis W, Brambilla E, Müller-Hermelink H, Harris C (Eds.): Pathology and Genetics of Tumours of the Lung, Pleura, Thymus and Heart. Volume 10; 3rd Edition; IARC Press, Lyon 2004, 188-195

Marx A, Chan J, Coindre J-M, Detterbeck F, Girard N, Harris N, Jaffe E, Kurrer M, Marom E, Moreira A, et al. (2015a): The 2015 World Health Organization Classification of Tumors of the Thymus: Continuity and Changes. J Thorac Oncol 10, 1383-1395

Marx A, Shimosato Y, Kuo T, Chan J, Travis W: Tumours of the Thymus; In: Travis W, Brambilla E, Burke A, Marx A, Nicholson A (Editors): WHO Classification of Tumours of the Lung, Pleura, Thymus and Heart. Volume 7; 4th Edition; World Health Organization, Geneva 2015b, 188-193

McCall C, Shi C, Klein A, Konukiewitz B, Edil B, Ellison T, Wolfgang C, Schulick R, Klöppel G, Hruban R (2012): Serotonin expression in pancreatic neuroendocrine tumors correlates with a trabecular histologic pattern and large duct involvement. Hum Pathol $\underline{43}$, 1169-1176

McCall C, Shi C, Cornish T, Klimstra D, Tang L, Basturk O, Mun LJ, Ellison T, Wolfgang CL, Choti M, et al. (2013): Grading of Well-differentiated Pancreatic Neuroendocrine Tumors Is Improved by the Inclusion of Both Ki67 Proliferative Index and Mitotic Rate. Am J Surg Pathol $\underline{37}$, 1671-1677

Modlin I, Lye K, Kidd M (2003): A 5-decade analysis of 13,715 carcinoid tumors. Cancer $\underline{\text { 97, }}$ 934-959

Modlin I, Moss S, Chung D, Jensen R, Snyderwine E (2008): Priorities for improving the management of gastroenteropancreatic neuroendocrine tumors. J Natl Cancer Inst 100, 1282-1289 
Modlin I, Kidd M, Filosso P-L, Roffinella M, Lewczuk A, Cwikla J, Bodei L, KolasinskaCwikla A, Chung K-M, Tesselaar M, Drozdov I (2017): Molecular strategies in the management of bronchopulmonary and thymic neuroendocrine neoplasms. $\mathrm{J}$ Thorac Dis $\underline{9}$, S1458-S1473

Moran C (2005): Primary neuroendocrine carcinomas of the mediastinum: review of current criteria for histopathologic diagnosis and classification. Semin Diagn Pathol 22, 223-229

Moran C, Suster S (2000a): Neuroendocrine carcinomas (carcinoid tumor) of the thymus. A clinicopathologic analysis of 80 cases. Am J Clin Pathol 114, 100110

Moran C, Suster S (2000b): Primary neuroendocrine carcinoma (thymic carcinoid) of the thymus with prominent oncocytic features: a clinicopathologic study of 22 cases. Mod Pathol $\underline{13}$, 489-494

Moran C, Suster S, Coppola D, Wick M (2009): Neuroendocrine carcinomas of the lung: a critical analysis. Am J Clin Pathol 131, 206-221

Nadler A, Cukier M, Rowsell C, Kamali S, Feinberg Y, Singh S, Law C (2013): Ki-67 is a reliable pathological grading marker for neuroendocrine tumors. Virchows Arch 462, 501-505

Niederle B, Pape U-F, Costa F, Gross D, Kelestimur F, Knigge U, Öberg K, Pavel M, Perren A, Toumpanakis C, et al. (2016): ENETS Consensus Guidelines Update for Neuroendocrine Neoplasms of the Jejunum and Ileum. Neuroendocrinology $\underline{103}, 125-138$

Nilsen G, LiestøI K, Van Loo P, Moen Vollan HK, Eide M, Rueda O, Chin S-F, Russell R, Baumbusch L, Caldas C, et al. (2012): Copynumber: Efficient algorithms for single- and multi-track copy number segmentation. BMC Genomics $\underline{13}, 591$

Oronsky B, Ma P, Morgensztern D, Carter C (2017): Nothing But NET: A Review of Neuroendocrine Tumors and Carcinomas. Neoplasia 19, 991-1002

Pan C-C, Jong Y-J, Chen Y-J (2005): Comparative genomic hybridization analysis of thymic neuroendocrine tumors. Mod Pathol $\underline{18}, 358-364$

Pelosi G, Papotti M, Rindi G, Scarpa A (2014): Unraveling Tumor Grading and Genomic Landscape in Lung Neuroendocrine Tumors. Endocr Pathol 25, 151164

Pelosi G, Pattini L, Morana G, Fabbri A, Faccinetto A, Fazio N, Valeri B, Sonzogni A (2017a): Grading lung neuroendocrine tumors: Controversies in search of a solution. Histol Histopathol $\underline{32}, 223-241$

Pelosi G, Sonzogni A, Harari S, Albini A, Bresaola E, Marchiò C, Massa F, Righi L, Gatti G, Papanikolaou N, et al. (2017b): Classification of pulmonary neuroendocrine tumors: new insights. Transl Lung Cancer Res $\underline{6}, 513-529$ 
Pelosi G, Bianchi F, Dama E, Simbolo M, Mafficini A, Sonzogni A, Pilotto S, Harari S, Papotti M, Volante M, et al. (2018): Most high-grade neuroendocrine tumours of the lung are likely to secondarily develop from pre-existing carcinoids: innovative findings skipping the current pathogenesis paradigm. Virchows Arch $\underline{472}, 567-577$

Peng W-X, Shibata T, Katoh H, Kokubu A, Matsuno Y, Asamura H, Tsuchiya R, Kanai Y, Hosoda F, Sakiyama T, et al. (2005): Array-based comparative genomic hybridization analysis of high-grade neuroendocrine tumors of the lung. Cancer Sci $\underline{96}, 661-667$

Phan A, Oberg K, Choi J, Harrison L, Hassan M, Strosberg J, Krenning E, Kocha W, Woltering E, Maples W (2010): NANETS consensus guideline for the diagnosis and management of neuroendocrine tumors: well-differentiated neuroendocrine tumors of the thorax (includes lung and thymus). Pancreas $\underline{39}$, 784-798

Reid M, Balci S, Saka B, Adsay NV (2014): Neuroendocrine Tumors of the Pancreas: Current Concepts and Controversies. Endocr Pathol 25, 65-79

Rieker R, Aulmann S, Penzel R, Schnabel P, Blaeker H, Esposito I, Morresi-Hauf A, Otto H, Hecker E, Dienemann H, et al. (2005): Chromosomal imbalances in sporadic neuroendocrine tumours of the thymus. Cancer Lett $\underline{223}, 169-174$

Rindi G, Klöppel G, Couvelard A, Komminoth P, Körner M, Lopes J, McNicol A-M, Nilsson O, Perren A, Scarpa A, et al. (2007): TNM staging of midgut and hindgut (neuro) endocrine tumors: a consensus proposal including a grading system. Virchows Arch 451, 757-762

Schaefer I-M, Zardo P, Freermann S, Marx A, Ströbel P, Fischer S (2013): Neuroendocrine carcinoma in a mediastinal teratoma as a rare variant of somatic-type malignancy. Virchows Arch 463, 731-735

Scheinin I, Sie D, Bengtsson $H$, van de Wiel M, Olshen A, van Thuij $H$, van Essen $H$, Eijk P, Rustenburg F, Meijer G, et al. (2014): DNA copy number analysis of fresh and formalin-fixed specimens by shallow whole-genome sequencing with identification and exclusion of problematic regions in the genome assembly. Genome Res 24, 2022-2032

Schmitt A, Riniker F, Anlauf M, Schmid S, Soltermann A, Moch H, Heitz P, Klöppel G, Komminoth P, Perren A (2008): Islet 1 (Isl1) expression is a reliable marker for pancreatic endocrine tumors and their metastases. Am J Surg Pathol 32, 420425

Schutz E, Akbari M, Beck J, Urnovitz H, Zhang W, Bornemann-Kolatzki K, Mitchell W, Nam R, Narod S (2015): Chromosomal instability in cell-free DNA is a serum biomarker for prostate cancer. Clin Chem 61, 239-248

Shoji T, Fushimi H, Takeda S, Tanio Y (2011): Thymic large-cell neuroendocrine carcinoma: a disease neglected in the ESMO guideline? Ann Oncol 22, 25352535 
Simbolo M, Mafficini A, Sikora K, Fassan M, Barbi S, Corbo V, Mastracci L, Rusev B, Grillo F, Vicentini C, et al. (2017): Lung neuroendocrine tumours: deep sequencing of the four World Health Organization histotypes reveals chromatinremodelling genes as major players and a prognostic role for TERT, RB1, MEN1 and KMT2D. J Pathol 241, 488-500

Sobin L, Gospodarowicz M, Wittekind C, International Union against Cancer (Editors): TNM classification of malignant tumours. 7th edition; Wiley-Blackwell, West Sussex 2010

Soga J, Yakuwa Y, Osaka M (1999): Evaluation of 342 cases of mediastinal/thymic carcinoids collected from literature: a comparative study between typical carcinoids and atypical varieties. Ann Thorac Cardiovasc Surg $\underline{5}$, 285-292

Sorbye H, Welin S, Langer S, Vestermark L, Holt N, Osterlund P, Dueland S, Hofsli E, Guren M, Ohrling K, et al. (2013): Predictive and prognostic factors for treatment and survival in 305 patients with advanced gastrointestinal neuroendocrine carcinoma (WHO G3): the NORDIC NEC study. Ann Oncol 24, $152-160$

Ströbel P, Zettl A, Shilo K, Chuang W-Y, Nicholson A, Matsuno Y, Gal A, Laeng RH, Engel P, Capella C, et al. (2014): Tumor genetics and survival of thymic neuroendocrine neoplasms: A multi-institutional clinicopathologic study. Genes Chromosomes Cancer $\underline{53}, 738-749$

Suster S, Moran C (1995): Thymic carcinoid with prominent mucinous stroma. Report of a distinctive morphologic variant of thymic neuroendocrine neoplasm. Am J Surg Pathol $\underline{19}$, 1277-1285

Swarts D, Claessen S, Jonkers Y, van Suylen R-J, Dingemans A-M, de Herder W, de Krijger R, Smit E, Thunnissen F, Seldenrijk C, et al. (2011): Deletions of 11q22.3-q25 Are Associated with Atypical Lung Carcinoids and Poor Clinical Outcome. Am J Pathol $\underline{179}, 1129-1137$

Swarts D, Van Neste L, Henfling M, Eijkenboom I, Eijk P, van Velthuysen M-L, Vink A, Volante M, Ylstra B, Van Criekinge W, et al. (2013): An exploration of pathways involved in lung carcinoid progression using gene expression profiling. Carcinogenesis $\underline{34}, 2726-2737$

Tang L, Basturk O, Sue J, Klimstra D (2016a): A Practical Approach to the Classification of WHO Grade 3 (G3) Well-differentiated Neuroendocrine Tumor (WD-NET) and Poorly Differentiated Neuroendocrine Carcinoma (PD-NEC) of the Pancreas. Am J Surg Pathol $\underline{40}, 1192-1202$

Tang L, Untch B, Reidy D, O'Reilly E, Dhall D, Jih L, Basturk O, Allen P, Klimstra D (2016b): Well-Differentiated Neuroendocrine Tumors with a Morphologically Apparent High-Grade Component: A Pathway Distinct from Poorly Differentiated Neuroendocrine Carcinomas. Clin Cancer Res 22, 1011-1017

Teh B (1998): Thymic carcinoids in multiple endocrine neoplasia type 1. J Intern Med $\underline{243}, 501-504$ 
Tetsu O, Hangauer M, Phuchareon J, Eisele D, McCormick F (2016): Drug Resistance to EGFR Inhibitors in Lung Cancer. Chemotherapy 61, 223-235

Tiffet O, Nicholson A, Ladas G, Sheppard M, Goldstraw P (2003): A Clinicopathologic Study of 12 Neuroendocrine Tumors Arising in the Thymus. Chest $\underline{124}, 141-$ 146

Travis W (2012): Update on small cell carcinoma and its differentiation from squamous cell carcinoma and other non-small cell carcinomas. Mod Pathol 25, S18-S30

Travis W, Brambilla E, Nicholson A, Yatabe Y, Austin J, Beasley MB, Chirieac L, Dacic S, Duhig E, Flieder D, et al. (2015): The 2015 World Health Organization Classification of Lung Tumors: Impact of Genetic, Clinical and Radiologic Advances Since the 2004 Classification. J Thorac Oncol 10, 1243-1260

Ullmann R, Petzmann S, Klemen H, Fraire A, Hasleton P, Popper H (2002): The position of pulmonary carcinoids within the spectrum of neuroendocrine tumors of the lung and other tissues. Genes Chromosomes Cancer $\underline{34}$, 78-85

Walch A, Zitzelsberger H, Aubele M, Mattis A, Bauchinger M, Candidus S, Präuer H, Werner M, Höfler H (1998): Typical and atypical carcinoid tumors of the lung are characterized by $11 \mathrm{q}$ deletions as detected by comparative genomic hybridization. Am J Pathol 153, 1089-1098

Weissferdt A, Kalhor N, Liu H, Rodriguez J, Fujimoto J, Tang X, Wistuba I, Moran C (2014): Thymic neuroendocrine tumors (paraganglioma and carcinoid tumors): a comparative immunohistochemical study of 46 cases. Hum Pathol $\underline{45}, 2463-$ 2470

Wick M, Scheithauer B, Weiland L, Bernatz P (1982): Primary thymic carcinomas. Am J Surg Pathol $\underline{6}, 613-630$

Yang Z, Tang L, Klimstra D (2011): Effect of tumor heterogeneity on the assessment of Ki67 labeling index in well-differentiated neuroendocrine tumors metastatic to the liver: implications for prognostic stratification. Am J Surg Pathol 35, 853860 


\section{Acknowledgements}

First and foremost, I would like to thank my doctorate supervisor Prof. Dr. med. Philipp Ströbel for giving me the opportunity to pursue a doctoral thesis under his mentorship and for allowing me to participate in such a rare subject matter and interesting project. I am grateful for the opportunity to partake in developing scientific papers and attending a pathology convention. I would also like to thank him for his arduous work of proofreading my doctorate thesis and providing scientific and experimental advice. I appreciate that Prof. Ströbel always made time for a quick meeting whenever questions arose, or next steps needed to be discussed.

My personal and warmest thanks go to Dr. med. Hanibal Bohnenberger for his tireless support and readiness to explain and discuss results and next courses of action. He shared his expertise with me, guided me through technical approaches and supported me during the writing process at any time.

Furthermore, I would like to acknowledge Stefan Küffer for his technical assistance during the preparation and conduction of experiments and for performing nextgeneration sequencing for this study. He always made himself available for questions. I would also like to acknowledge Jennifer Appelhans and Mercedes Martin-Ortega for their hard work and for carrying out the immunohistochemical stains.

This study was possible due to the cooperation with Targos Molecular Pathology $\mathrm{GmbH}$ and Chronix Biomedical.

Lastly, I would like to thank the entire Institute of Pathology. Without their commitment, this study would not have been possible. 